\title{
ELECTRONIC DNA DETECTION AND DIAGNOSTICS
}

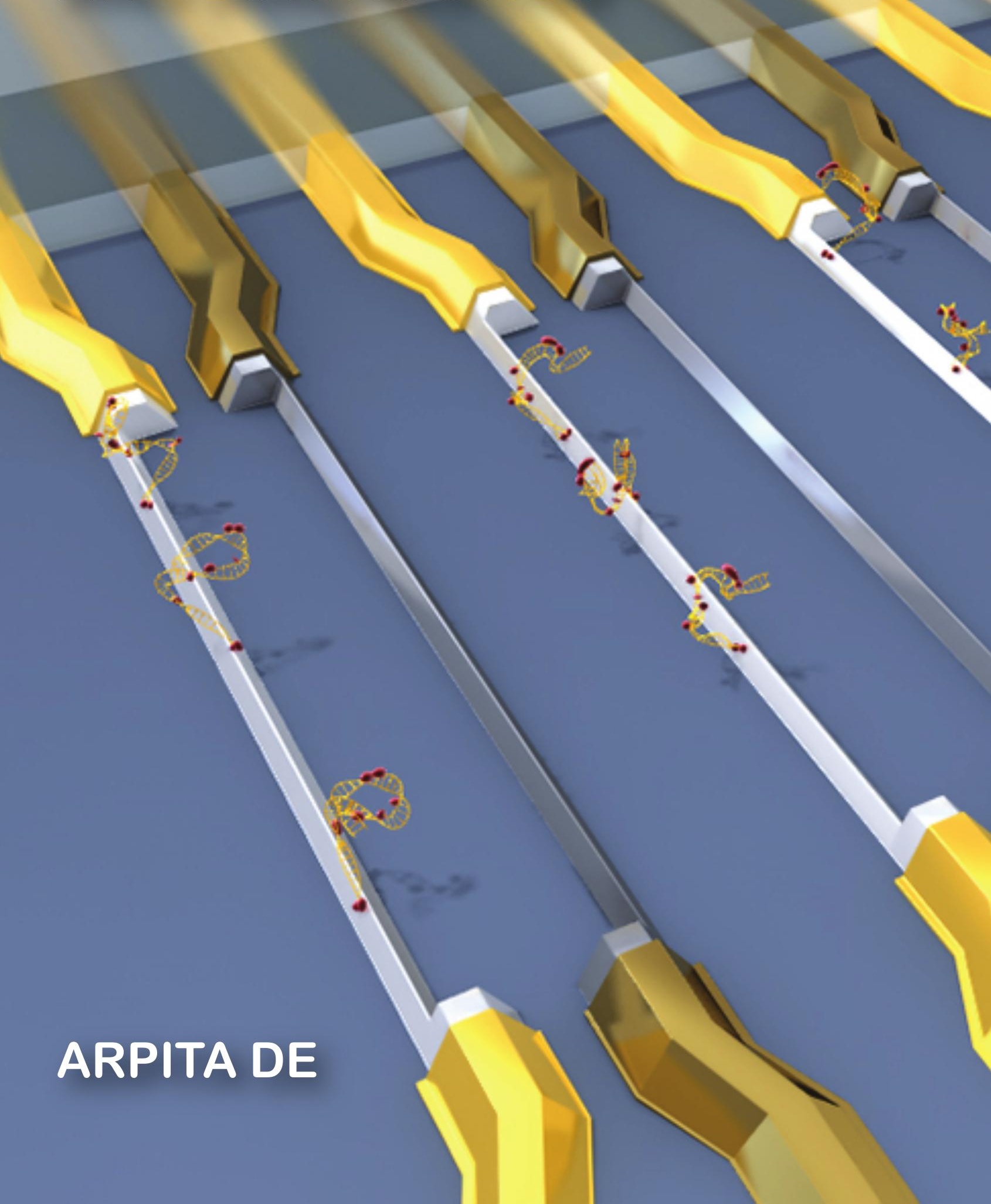




\section{UNIVERSITEIT TWENTE.}

\section{Electronic DNA Detection and}

\section{Diagnostics}

Arpita De 
The research described in this thesis has been carried out in the BIOS/ Lab-on-aChip group at the MESA+ Institute for Nanotechnology, University of Twente, Enschede, The Netherlands. The project was financially supported by a private foundation in The Netherlands.

Committee members:

\section{Chairman}

Prof. dr. ir. A.J. Mouthaan

University of Twente

\section{Promotor}

Prof. dr. ir. A. van den Berg University of Twente

\section{Assistant promotor}

Dr. E.T. Carlen

University of Twente and

University of Tsukuba

\section{Members}

$\begin{array}{ll}\text { Prof. dr. H.M. Pinedo } & \text { University of Twente } \\ \text { Prof. dr. Han Gardeniers } & \text { University of Twente } \\ \text { Prof. dr. ir. Wilfred van der Wiel } & \text { University of Twente } \\ \text { Prof. dr. ir. J.M.J den Toonder } & \text { Eindhoven University of } \\ & \text { Technology } \\ \text { Prof. James R. Heath } & \text { California Institute of } \\ & \text { Technology }\end{array}$

Title: Electronic DNA Detection and Diagnostics

Author: Arpita De

ISBN: 978-90-365-0263-4

DOI: $10.3990 / 1.9789036502634$.

Publisher: Wohrmann Print Service, Zutphen, The Netherlands

Cover: 3D nanowire device and MBD capture images by Nymus3D

Copyright (C 2013 by Arpita De, Enschede, The Netherlands 


\title{
ELECTRONIC DNA DETECTION AND DIAGNOSTICS
}

\section{DISSERTATION}

\author{
To obtain \\ the degree of doctor at the University of Twente \\ on the authority of the rector magnificus, \\ prof. dr. H. Brinksma, \\ on account of the decision of the graduation committee, \\ to be publicly defended \\ on Friday the $30^{\text {th }}$ of August 2013 at 14:45 hrs
}

by

\section{Arpita De}

born on February $24^{\text {th }}, 1984$

in West Bengal, India 
Dit proefschrift is goedgekeurd door

Promotor: Prof. dr. ir. Albert van den Berg Assistant promotor: Dr. Edwin T. Carlen 
To My Parents 



\section{Table of Contents}

Chapter 1 ......................................................................................................

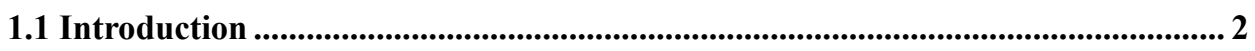

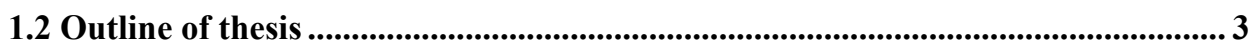

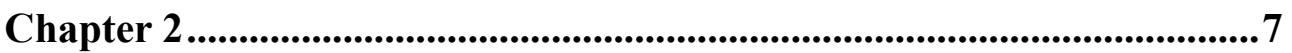

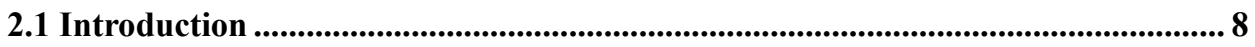

2.2 Conventional CRC screening ……………………........................................................ 8

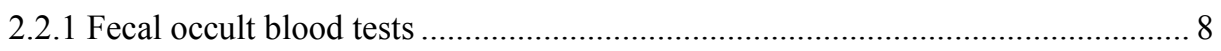

2.2.2 Fecal immunochemical test......................................................................... 9

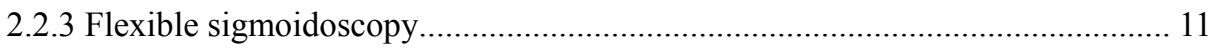

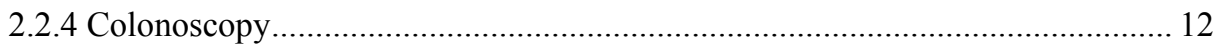

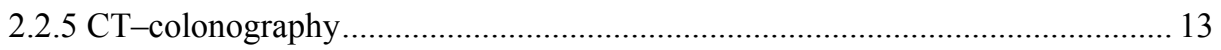

2.2.6 Screening data assessment ........................................................................ 13

2.3 Emerging CRC screening ............................................................................................ 14

2.3.1 Imaging techniques: Capsule endoscopy......................................................... 14

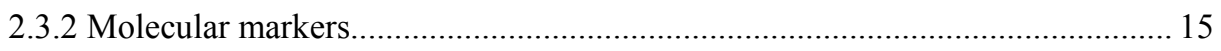

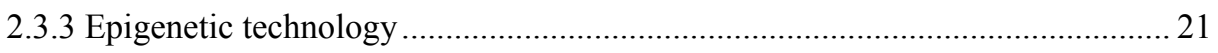

2.4 Nanopill: The next generation of CRC screening assay ......................................... 24

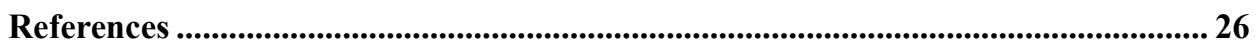

Chapter 3 ..................................................................................................35

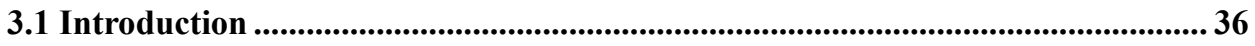

3.2 Nanopill automated diagnostic bioassay.................................................................. 39

3.3 Nanopill system and components ...................................................................... 40

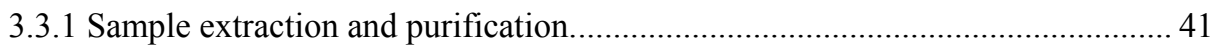

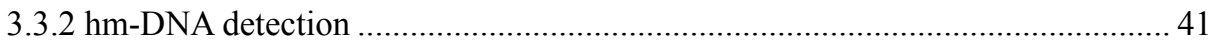

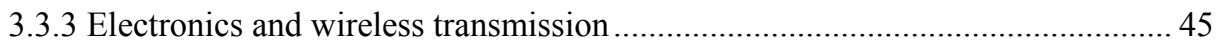

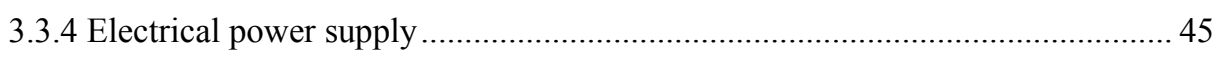




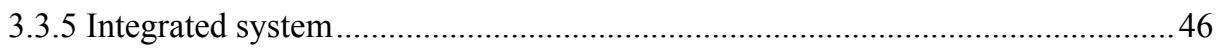

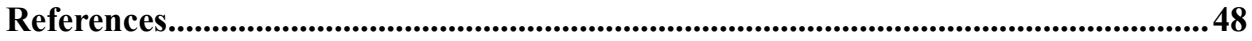

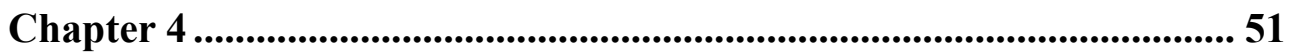

Target enrichment using microfluidic solid phase extraction ................51

4.1 Introduction......................................................................................................................52

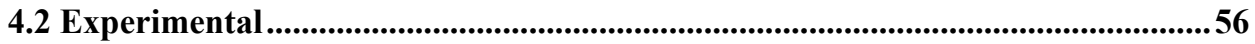

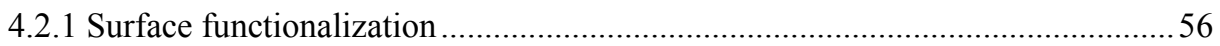

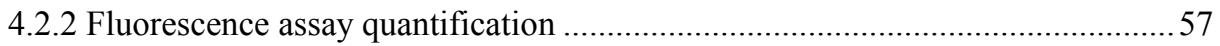

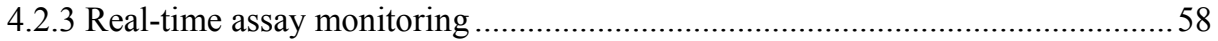

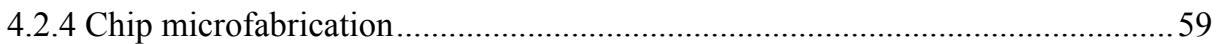

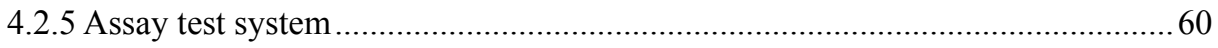

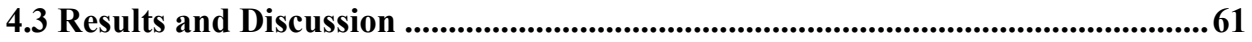

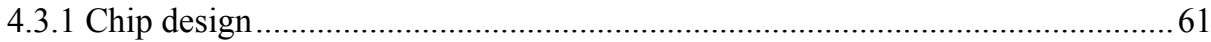

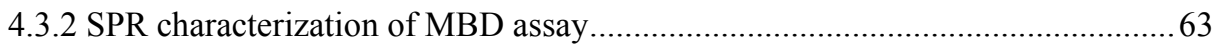

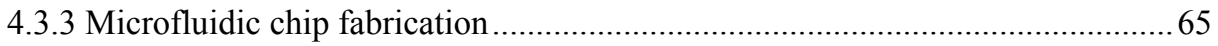

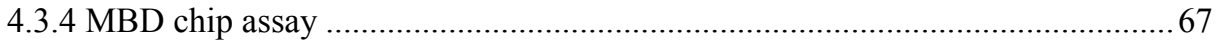

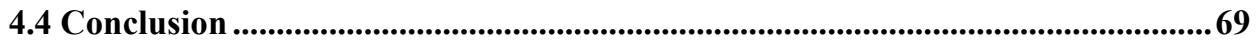

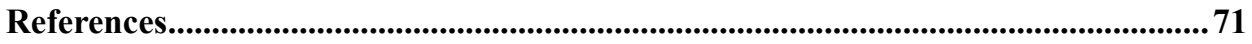

Chapter 5 .............................................................................................. 75

5.1 Introduction.............................................................................................................................. 76

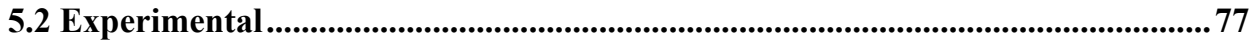

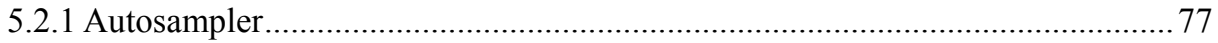

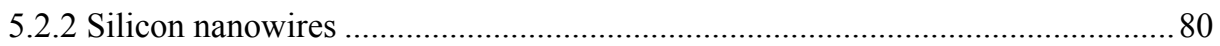

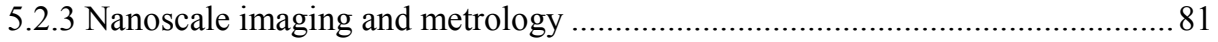

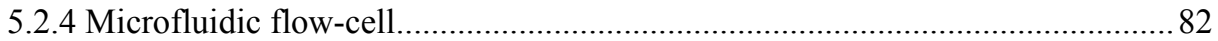

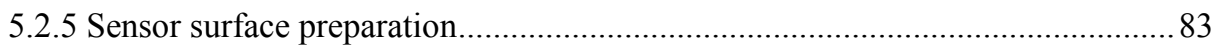

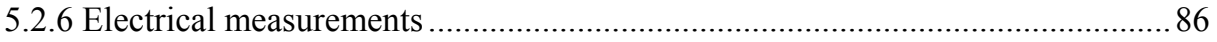

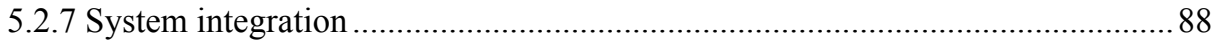

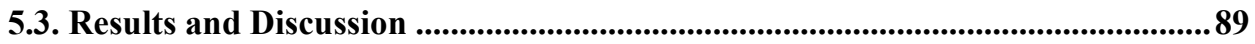




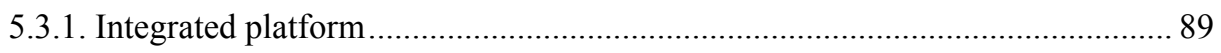

5.3.2 Silicon nanowire sensor sample flowrate dependence ................................... 90

5.3.3 Si-NW operation and characterization ....................................................... 91

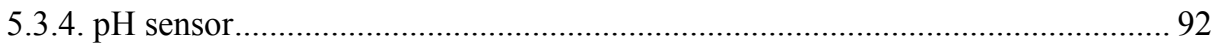

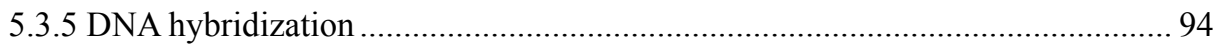

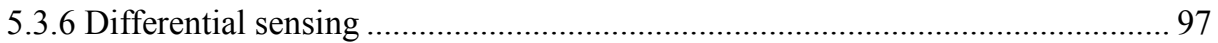

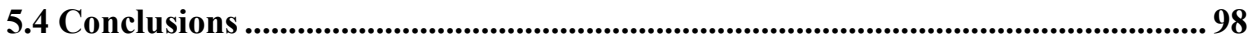

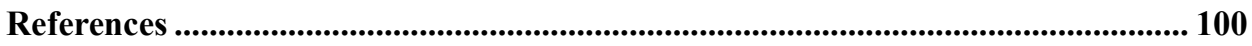

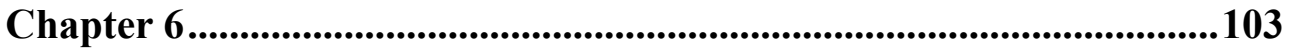

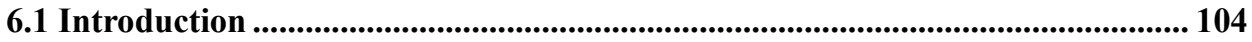

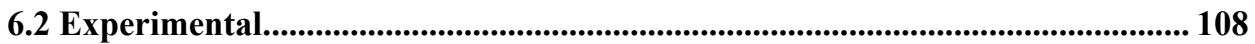

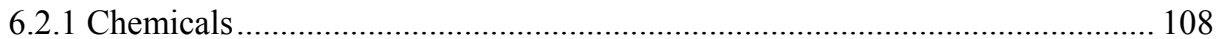

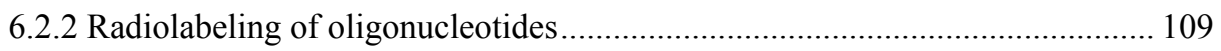

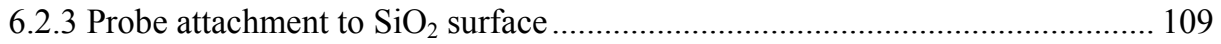

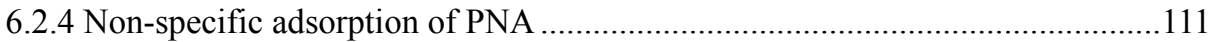

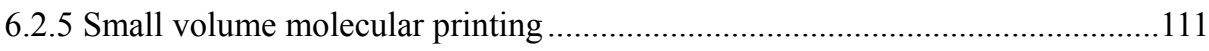

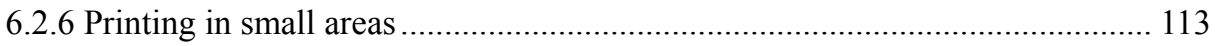

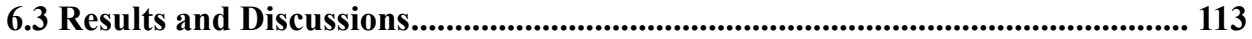

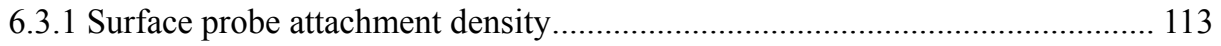

6.3.2 Non-specific adsorption of PNA on surfaces ............................................... 115

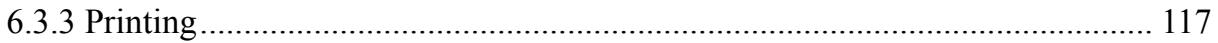

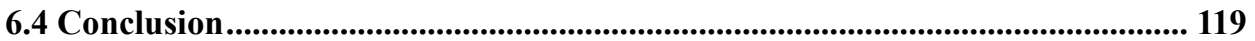

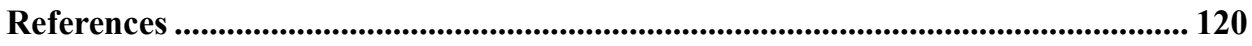

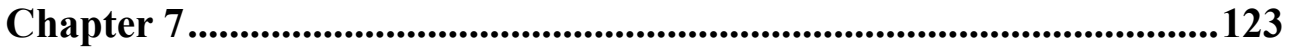

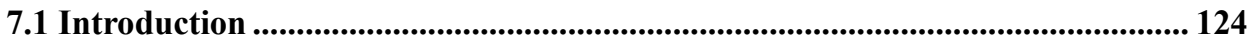

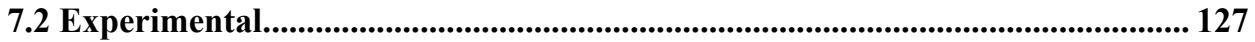

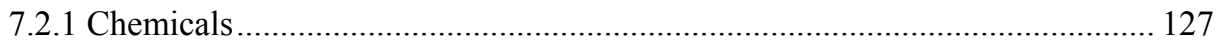

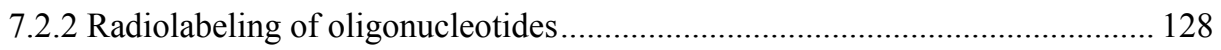

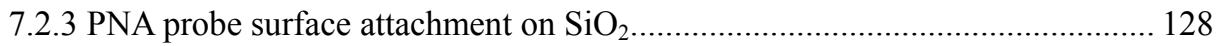

7.2.4 Surface plasmon resonance measurements .................................................. 130 


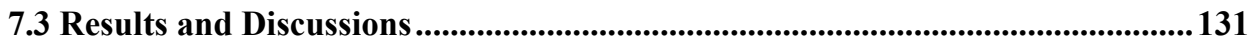

7.3.1 Vertical and horizontal PNA-DNA hybridization on $\mathrm{SiO}_{2}$ surface ................... 131

7.3.2 Vertical and horizontal PNA-DNA hybridization detection: SPR measurements

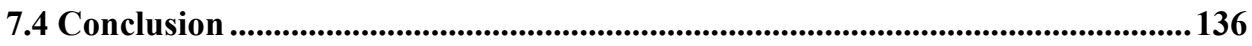

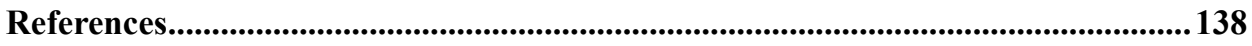

Chapter 8 .................................................................................. 141

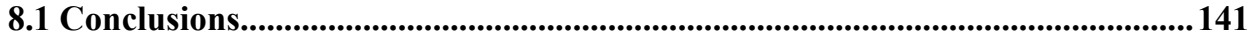

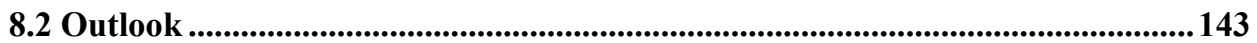

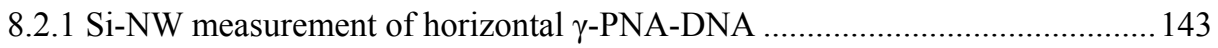

8.2.2 Combined hm-DNA enrichment and electronic DNA detection....................... 144

Samenvatting............................................................................................... 146

Acknowledgment ............................................................................................... 149

Appendix......................................................................................................... 152

List of Publications .......................................................................................... 154 



\section{Chapter 1}

\section{Project aims}

This chapter gives a brief overview about the background and motivation for the work being presented in this thesis and describes what follows in each individual chapters. 


\subsection{Introduction}

Colorectal cancer (CRC) is a highly prevalent disease, affecting approximately 1.23 million patients, above the age of 50, both men and women, globally every year and accounts for almost $10 \%$ of all cancer cases. CRC is characterized by an extended preclinical stage, with the advancement from early adenoma to invasive cancer over prolonged time of 30 years. The CRC characteristics necessitate population screening more than any other malignancy to prevent high CRC mortality rates. Current CRC screening tests include the fecal occult blood test (FOBT) and fecal immunochemical test (FIT), which are noninvasive and inexpensive assays that detect microscopic amounts of blood in stool that is shed by a considerable proportion of advanced adenomas and a majority of cancers. These tests either detect heme (FOBT) or human globin (FIT), parts of the hemoglobin molecule in stool. Patients who test positive are then typically referred for colonoscopy, which is considered a highly reliable CRC cancer prevention tool that is time consuming and expensive.

The CRC tests based on the detection of blood in stool can be inconclusive due to a high rate of false-positive results since the blood loss can arise from other sources, such as colorectal neoplasia, diverticulitis, vascular lesions, or hemorrhoids. A negative test has a limited negative predictive value for colorectal neoplasia in symptomatic patients. However, the blood-in-stool test offers several advantages, from a public health perspective, as they are inexpensive, simple to distribute, easy to use, stable in dry form and enable the assessment of multiple fecal samples, which reduces sampling error. Importantly, the tests can be used to pre-screen the general population and only patients that test positive are selected for colonoscopy. Sigmoidoscopy and colonoscopy are invasive and expensive, but are capable of early detection and can also prevent CRC by precursor removal. Colonoscopy, is the current gold standard for evaluation and screening for colorectal cancer although it is invasive and deals with bowel preparation problems that varies from patient to patient. In addition, often endoscopy resources for huge population-based screening programs are not available added to the inherent reluctance of patients to undergo colonoscopy due to its alleged inconvenience, discomfort, or embarrassment. Recently, a less burdensome capsule-based endoscopic method, such as the Pillcam (Pillcam Colon), which records images of the bowel as the pill travels down the intestinal tract, is undergoing clinical trials for approval. 
However its sensitivity is reduced for colonic lesion detection in comparison to conventional colonoscopy. Hence the idea to develop the Nanopill was borne, by Prof Dr. Bob Pinedo, as an ingestible smart diagnostic pill that could potentially replace the void of a less invasive robust and reliable early colorectal cancer detection system based on the specific detection of early molecular cancer markers close to the sight of neoplasms by sampling intestinal fluid inside the gastrointestinal tract.

The Nanopill project aims at developing an ingestible smart diagnostic pill for the non-invasive early detection of colorectal cancer. The patient swallows the Nanopill, and the pill subsequently travels to the large intestine, where intestinal fluid is sampled by a miniaturized lab-on-a-chip microfluidic system contained within the Nanopill. Although the Nanopill diagnostic pill can be configured to detect many different caner markers, this project considers freefloating hyper-methylated tumor DNA from cancer cell bursts, which is gaining acceptance as a reliable marker of cancer. The sampled cell-free DNA in the intestinal fluid is then purified and pre-concentrated inside the microfluidic chamber, and aspired into the Nanopill. Inside the pill a specific DNA sequence is detected at miniature silicon nanowire ( $\mathrm{Si}-\mathrm{NWs}$ ) sensors. $A P C$ is a tumorsuppressor gene involved in colorectal cancer. When both alleles of the $A P C$ gene are inactivated by mutation, polyps form in the colon. Other mutations in tumor suppressor genes like ras, p53, characterize malignant carcinoma. Hypermethylation in the promoter regions of these tumor suppressor genes start as a mechanism for gene silencing. . We want to test for the abnormality in these sequences in a miniaturized assay inside the Nanopill. When a positive detection event occurs, a microsensor contained inside the Nanopill, would report a response as a wireless signal to a modern smart-phone, for example.

The primary goal of this thesis has been to design, implement and characterize the DNA detection assay using Si-NW biosensors, and the hypermethylated DNA purification and pre-concentration microchip for specific DNA detection using a two-step assay, which would form the core of Nanopill detection system.

\subsection{Outline of thesis}

Chapter 2 gives a general overview on the current state of the art techniques for colorectal cancer screening, the biology of colorectal cancer, molecular markers 
for colorectal cancer, emerging epigenetic markers, technologies for detecting epigenetic markers and the subsequent need for a modern testing system with higher accuracy for early detection of cancer marker using epigenetic markers such as hyper-methylated DNA for non-invasive early colorectal cancer detection such as the Nanopill system.

Chapter 3, describes the concept and components of the Nanopill system. It provides the tangible details of the actual realization of an ingestible smart pill in form and function with the various interconnected chambers that would communicate in an automated sample-in and answer-out system operation.

Chapter 4 describes the design and implementation of the solid phase capture-elution microchip for the capture of hyper-methylated DNA (hm-DNA) using specific methyl-binding domain (MBD) proteins and its subsequent elution. The hm-DNA capture with MBD-proteins against control non-hm-DNA and the capture-elution protocol is demonstrated qualitatively with a surface plasmon resonance biosensor. The performance of MBD-hm-DNA captureelution microchip has been quantified using calibrated fluorescence-based assay. The saturation limit and pre-concentration ability of the capture-elution microchip is demonstrated.

Chapter 5 describes the electronic detection of DNA using Si-NW biosensors integrated into an automated microfluidic platform. Si-NWs detect the change in surface potential induced by surface charge at the gate oxidesurface. In order to detect the intrinsic negative charge of target DNA molecules, the gate-oxide surface is conjugated with a complementary capture moiety. Since DNA is a negatively charged molecule, DNA-DNA hybridization prefers a high ionic strength buffer, thus shielding the negative charge and minimizing like charge repulsion. For Si-NW biosensors, DNA-hybridization detection is achieved using high-affinity synthetic anionic peptide nucleic acid (PNA) probe molecule that can readily form a DNA-PNA duplex in a low ionic strength buffer, which reduces charge screening of the target DNA. Si-NW measurements in flowing low ionic strength buffers are sensitive to flow rate variations and erroneous sensor responses from sample switching due to streaming potential have been addressed. In this chapter we describe an integrated Si-NW microfluidic auto-sampler that eliminates sensor responses induced by sample switching and is suitable for real-time PNA-DNA hybridization measurements 
and is compatible with kinetic binding parameter estimation. A differential SiNW sensor configuration has been implemented to reduce the sensor signal drift and improve detection sensitivity.

Chapter 6 describes the details necessary to form a good DNA hybridization probe layer with PNA probe molecules with regard to surface attachment schemes, surface density, and issues of non-specific adsorption of probe molecules using fluorescence and radioactivity assays. These details for probe layer formation appear innocuous, but their knowledge is fundamental for reliable DNA hybridization measurement using Si-NW biosensors.

Chapter 7 describes a new horizontally tethered PNA probe concept and the results are compared to conventional vertically tethered PNA probes using real-time PNA-DNA hybridization surface plasmon resonance biosensor measurements and radioactivity labeled assays.

Finally, Chapter 8 describes a summary of the presented work in the thesis as a conclusion together with an outlook for the next steps to be considered in the future. 
Chapter 1 


\section{Chapter 2}

\section{Colorectal cancer screening technology: Current strategies and new directions}

This chapter gives a general overview of the present status of available techniques for colorectal cancer screening with details of the fecal occult blood test, fecal immunochemical tests, sigmoidoscopy, colonoscopy, CT-colonoscopy, and emerging molecular marker assays, and specifically epigenetic markers for early colorectal cancer detection and why there is a need to develop the noninvasive Nanopill based on molecular markers for early colorectal cancer detection. 


\subsection{Introduction}

The colorectal cancer (CRC) mortality rate is high. A 2012 report from the United States estimated that 143,460 persons were diagnosed with CRC, out of which 51,690 deaths were caused by CRC. ${ }^{1}$ Today, clinicians and patients have a large range of CRC screening detection tests, such as colonoscopy, flexible sigmoidoscopy, CT colonography (CTC), and stool tests. CRC incidence and mortality have experienced a significant decline in large part due to the enhanced screening efforts. The available CRC screening tests merge both early detection tools with cancer-prevention tools. Early detection tools include the fecal occult blood test (FOBT) and fecal immunochemical test (FIT), which are noninvasive and inexpensive assays for detecting microscopic amounts of blood in stool arising for a considerable proportion from advanced adenomas that most cancers produce. The FOBT detects heme and the FIT detects human globin FIT, which are both part of the hemoglobin molecule. Patients who test positive using one of these pre-screening tests are then typically referred for endoscopy, which is classified as a cancer-prevention tool. Sigmoidoscopy and colonoscopy are invasive and expensive, but are capable of early detection and can also prevent CRC by precursor removal. ${ }^{2}$ In this chapter, we will provide a summary of the status of CRC screening tools, which is based primarily on three previous comprehensive CRC reviews, ${ }^{2-4}$ and reasons for developing the next generation cancer test, such as the Nanopill, for early warning cancer detection.

\subsection{Conventional CRC screening}

\subsubsection{Fecal occult blood tests}

The guaiac FOBT (gFOBT) is named after the paper used in the device, which is from Guaiacum trees. The tests qualitatively detect microscopic amounts of heme in the stool. ${ }^{5}$ The principle of testing involves exposure of the fecal smear on the guaiac material to hydroperoxidase resulting in oxygenation of guaiac, which is confirmed by a blue color change in a heme catalyzed process, which is shown in the example in Fig. 1. The gFOBT is a very simple test that is a sample-in and answer-out system, and suitable for large population based screening programs. Most importantly, the tests can be used to pre-screen the population for colonoscopy by selecting patients who test positive for referral as required. However clinicians are biased towards diagnosing more symptomatic 
patients rather than taking up large population screening and averse to huge number of fecal handling. ${ }^{5}$
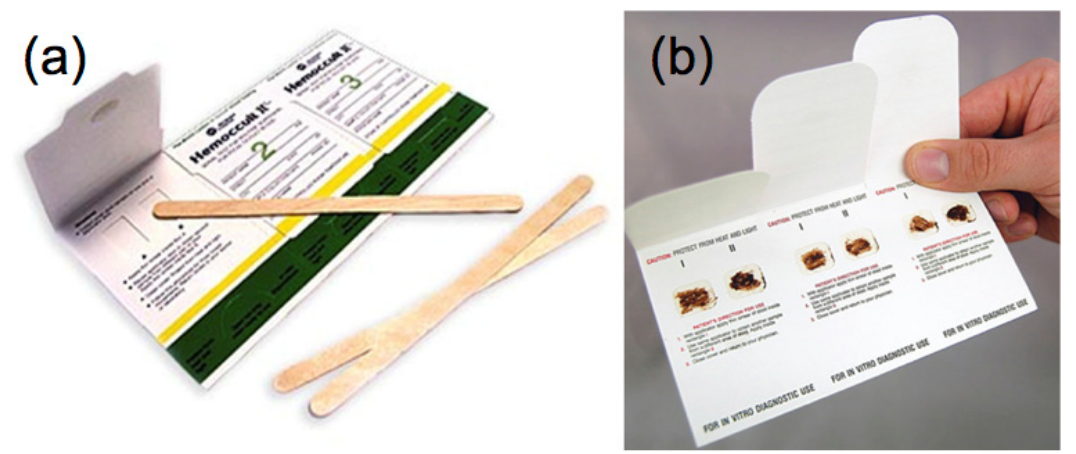

Figure 1. A fecal occult blood testing (FOBT) kit (a) include instructions, test paper, and sample collection sticks (b) manual result readout from sample smears. ${ }^{6,7}$

The shortcomings of the gFOBT include its limited sensitivity for cancer detection, manual operation, and reader-dependency. ${ }^{2}$ Another disadvantage of gFOBT is related to the chemical stability of heme from nonhuman sources, which can increase the false-positive detection rate. ${ }^{8} 9$ In summary, the FOBT is robust and has been evaluated in several long-term randomized tests, which showed that repeated biennial screening leads to a reduction in CRC mortality. Although the gFOBT test is limited, it does proved value given the prevalence of CRC in Western countries, as well as countries with limited colonoscopy resources and laboratory budgets.

\subsubsection{Fecal immunochemical test}

The fecal immunochemical test (FIT) is based on detection of human globin by means of an antibody-based assay. The FIT can be automated and used for the sensitive quantitative detection of microscopic amounts of globin the stool samples. ${ }^{10}$ The FIT is available as a qualitative assay, with either a positive or negative result, and as a quantitative assay that provides the hemoglobin concentration in the stool sample, as shown in the two application types of Fig. $2 .^{10,11}$ The FIT is advantageous over the gFOBT mainly due to the superiority of globin assay. Globins are human-specific, and do not cross-react with traces of dietary non-human blood in stool. The FIT can readily rule out contamination from upper gastrointestinal sources as globin is rapidly degraded during its passage through the gastrointestinal tract ${ }^{12,13}$ thus lowering the FIT sensitivity for lesions in the proximal colon, or so-called right-sided lesions. 
FIT screening is characterized by a number of shortcomings, such as globin degradation, temperature sensitivity, and lack of standardization problems. As previously described, globin is degraded as it passes through the colon, reducing sensitivity for proximal colon (right-sided lesions) than distal colon or rectum (so-called left-sided lesions).
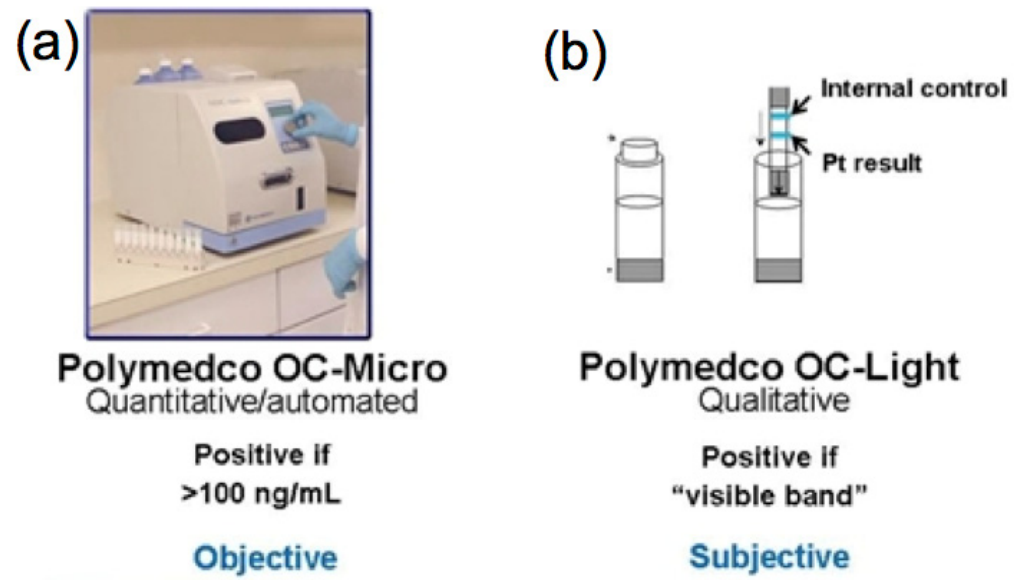

Figure 2. Comparison of a quantitative and a qualitative FIT tests (a) automated assay with objective quantitative results (b) semi-automated with subjective qualitative result from the colored band on the immuno-strip. ${ }^{14}$

The FIT kits are temperature sensitive, similarly to the gFOBT, since the ironcontaining heme-pyrrole ring can degrade in warm temperatures. ${ }^{15}$ This effect is more pronounced with the FIT compared to the gFOBT, and accumulates a 70\% of hemoglobin within $24 \mathrm{~h}$ at $30^{\circ} \mathrm{C}^{16}$ The temperature limitation of the FIT and gFOBT is problematic and a barrier for CRC screening in warmer climates. Currently, manufacturers are developing other FIT buffer solutions that can overcome this problem. ${ }^{10}$ Another disadvantage of the FIT is the lack of standardization of hemoglobin concentrations among different FIT kits. ${ }^{17}$ Although this lack of standardization does not constitute an intrinsic limitation of any individual test, it does represent a limitation when comparing results obtained from different studies. Such comparisons are important for reporting the standardized unit of hemoglobin concentration per gram of feces. Finally, the cost of the average FIT kit, which includes a sampling device and developing chemicals, is high compared to the gFOBT. In essence, FITs are very simple and expensive. Their high cost has limited the high volume use for high-throughput assays. $^{2}$ 


\subsubsection{Flexible sigmoidoscopy}

The gFOBT has been the primary CRC screening technique with supported evidence of randomized trials with end points of cancer incidence and mortality. $^{2}$ Four large prospective randomized trials were conducted which compared flexible sigmoidoscopy screening with no screening, ${ }^{18-21}$ which revealed that over a follow-up period of more than $11 \mathrm{yrs}$, the CRC incidence reduced by $21-23 \%$, and CRC mortality reduced to the highest level of $31 \%$. These results established the use of flexible sigmoidoscopy as a CRC screening tool and leading to the reduction of CRC incidence and an approximate two-fold greater reduction of CRC mortality than biennial gFOBT screening. ${ }^{2,18-21}$. These large scale trails were carried out in Norway, ${ }^{18} \mathrm{UK},{ }^{19}$ Italy $^{20}$ and USA.

In this technique, a flexible endoscope is used to image the distal colon with a regular forward-viewing video-colonoscope, as shown in Fig. 3(a), which either reaches the descending colon or the splenic flexure, as (Fig. 3(b)). The usual bowel preparation for screening purposes consists of a single phosphate enema that most screenees can conduct at home. The enema is usually combined with oral bowel preparation using commercially available solutions, e.g., Prunacolon ${ }^{\circledR}$ (Nycomed BV, Hoofddorp, The Netherlands).
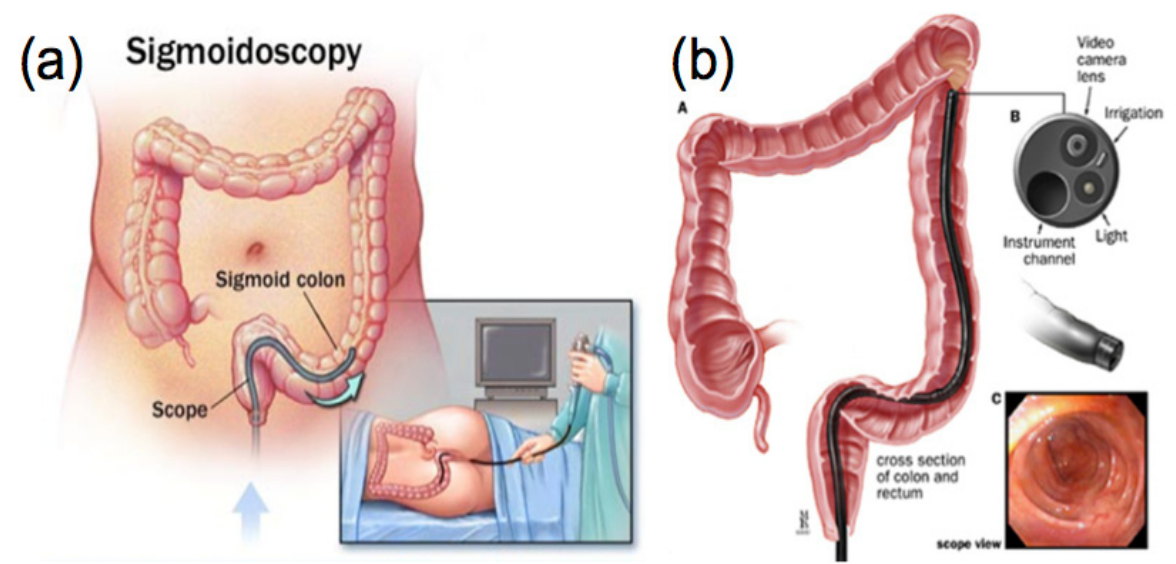

Figure 3. Flexible sigmoidoscopy exam. (a) The doctor inserts a sigmoidoscope into the rectum to check for abnormalities in the lower colon. ${ }^{22,}{ }^{23}$ (b) Detailed description of endoscope.

Small polyps, up to $9 \mathrm{~mm}$ in diameter, can be removed during sigmoidoscopy. ${ }^{2}$ The procedure is usually performed with sedation. Candidates with large, or more than three small polyps, require a complete colonoscopy. The major 
shortcomings associated with sigmoidoscopic screening include variable uptake, failure to detect proximal neoplasias, invasive, and expensive.

\subsubsection{Colonoscopy}

The gold standard for CRC screening is colonoscopy, based on its ability to visualize the complete colon as well as directly remove neoplastic lesions and precancerous growths, or polyps. ${ }^{24}$ In colonoscopy, a flexible, regular forwardviewing video-colonoscope is used to image the entire colon, as shown in the different stages in Fig. 4. 'The usual bowel preparation for screening purposes consists of oral complete bowel lavage, which most screenees take at home. Colonoscopy is usually performed after intravenous administration of a benzodiazepine with or without an analgesic. In some countries, such as France, colonoscopy is often performed under complete anesthesia, whereas other countries, such as Norway, colonoscopy is often performed without any sedation., ${ }^{2}$

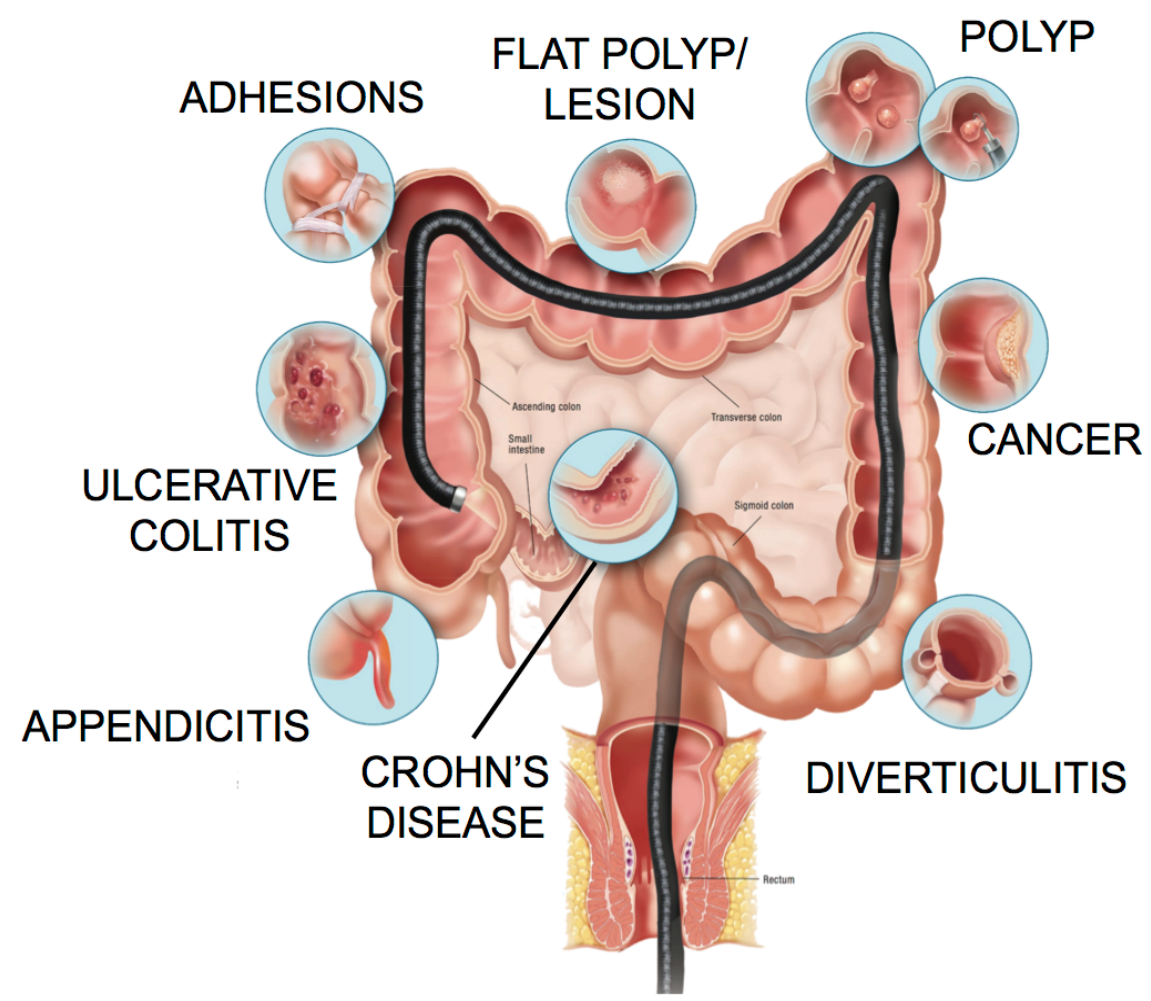

Figure 4. Colonoscopy: viewing the entire colon with a forward flexible endoscope which can examine all abnormalities associated with the inside of the colon. ${ }^{25}$ 
Colonoscopy is used for primary CRC screening in several countries such as the USA, Canada, Germany and Poland. ${ }^{26,} 27$ The colonoscopy screening data accentuates the need for quality assurance in colonoscopy, for primary and secondary screening, as well as surveillance. There have been reports of occurrence of post-endoscopy cancer, where the cancer risk is inversely proportional with the baseline adenoma detection rate, ${ }^{26,28}$ which is generally due to complications based on the experience of the endoscopist ${ }^{29}$ and on the quality of the bowel preparation. ${ }^{30}$ 'This has led to a range of guidelines for implementing quality assurance in screening colonoscopy with recommendations, including monitoring of key indicators such as adequacy of bowel preparation, caecal intubation, adenoma detection, adequacy of surveillance and interval cancers. ${ }^{2,24,31-33}$

\subsubsection{CT-colonography}

Computed tomography (CT) colonography uses CT x-ray images of the colon and rectum to identify polyps and lesions, and is typically used when a conventional colonoscopy test is not possible. Usually CT-colonography is performed without sedation and limited bowel preparation is necessary. A typical protocol procedure involves non-cathartic bowel preparation with a lowfiber diet for 1 day prior to the examination, in addition to, two oral doses of an iodinated contrast agent, such as ioxithalamate (Telebrix Gastro, Guerbet). Adequate bowel distension is important for proper imaging. It is possible to detect $70-100 \%$ of the advanced neoplasias as detected by colonoscopy using CT-colonography. ${ }^{34-37}$ The detection sensitivity for cancer is very high and the incidence of cancer 5 years after a negative CT-colonography is low, ${ }^{38}$ following treatment with a skilled radiologist or technician, ${ }^{39}$ However, CTcolonography is still more expensive than other screening modalities both for primary screening ${ }^{40}$ as well as for secondary screening after a positive FOBT because of the substantial costs of the procedure and the subsequent need for colonoscopy to confirm and remove lesions. ${ }^{41-43,44}$

\subsubsection{Screening data assessment}

All of the screening techniques and early detection methods generate a plethora of patient data. The number of screening strategies seems to be endless, with a huge number of variables which can be anything from age, screening interval, cut-off value for colonoscopy referral, sex, family history of CRC, seasonal 
temperatures, in combination with sampling and analysis intervals, previous screening test results, which are considered while evaluating colonoscopy referral. ${ }^{5,45,46}$ Numerous CRC mathematical models have been developed and compared for the efficient analysis of the screening conditions. ${ }^{47}$ Randomized FOBT and flexible sigmoidoscopy trial data have been used to validate the models. $^{46}$ Large-scale gFOBT and FIT screening programs have been conducted, in terms of life-years saved, cost and colonoscopy demand. A quantitative FIT with a low cut-off value was determined to be more costeffective than any other gFOBT or FIT strategy. ${ }^{46}$

\subsection{Emerging CRC screening}

\subsubsection{Imaging techniques: Capsule endoscopy}

In efforts to improve CRC screening with less patient burden, and enhanced neoplasia detection, the field of colon imaging is rapidly evolving with methods such as conventional endoscopes - a transparent plastic cap is placed on a retrograde viewing device on the tip of the scope to improve visualization, ${ }^{48,49}$ or the colon immersed in water while the endoscope is introduced. ${ }^{50}$ In other techniques, modified endoscopes are used to enlarge the field of visualization using, e.g., wide-angle view, ${ }^{51}$ or by adding side-viewing lenses to the conventional forward view endoscope. The latest in the line of improvement has been introducing an ingestible capsule endoscope, ${ }^{52}$ with a 172 -degree video imager at each end, as shown in Fig. 5. The capsule travels through the colon by peristalsis, and transmits images to an external data recorder carried by the patient. The capsule is discarded with a bowel movement. This type of imaging demands a very clean colon and passage of the capsule within $8-10 \mathrm{~h}$; thus requiring colon preparation with lavage combined with repeated intake of a prokinetic drug. However, none of these new techniques have been assessed in the screening setting and no reliable data are available with regards to efficacy of detecting CRC and cost-effectiveness.

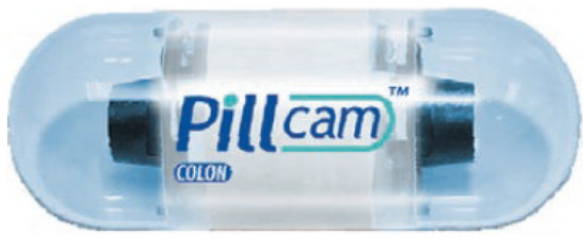

Figure 5. The PillCam COLON Capsule: The capsule is shown at approximately twice its actual dimensions. ${ }^{53}$ 
A study in the non-screening setting compared capsule endoscopy and colonoscopy in 109 patients and reported $88 \%$ sensitivity and $95 \%$ specificity for the detection of neoplastic lesions greater than $10 \mathrm{~mm}$ for the capsule. ${ }^{54}$ Although these results are encouraging, further confirmation in a larger screening series is required. The general use of capsule endoscopy for primary population screening is limited by the cost of the capsule, which is currently considerably higher than FOBT and colonoscopy in most countries. The reading and interpretation of the images can be tedious, and when combined with the need for colon lavage, and prokinetic drugs after capsule intake, reduce its convenience as a screening method. ${ }^{54,55}$ Capsule endoscopy has not yet received approval in many countries and more exhaustive testing is required. ${ }^{43}$

\subsubsection{Molecular markers}

Colon cancer can be described by the well-characterized morphological stages such as polyps, benign adenomas, and carcinomas, as summarized in Fig. 6. The intermediate stages can be individually isolated by a surgeon, allowing studies of mutations that occur in each of the morphological stages. Many reports have accumulated with evidence revealing that colon cancer arises due to a series of mutations occurring in a well-defined order. ${ }^{56}$

Consistently, the very first step in colon carcinogenesis appears to be the loss of a functional $A P C$ gene, resulting in the formation of polyps (precancerous growths) on the inside of the colon wall. An order of acquiring sequential mutations exists in colorectal cancer. However not every colon cancer, acquires all of the mutations. Hence it is possible that different combinations of mutations may result in the same phenotype. ${ }^{56}$

The majority of the cells in a polyp report the same one, or two inactivating mutations in the $A P C$ gene. Those cells propagate as clones of the original cell in which the original mutation occurred. The $A P C$ gene is a tumor-suppressor gene (TSG). When both alleles of the $A P C$ gene undergo an inactivating mutation, polyps can form, otherwise cells with one wild-type $A P C$ gene can express enough normal functional APC protein. Similar to most TSG,

$A P C$ encodes a check-point protein that is responsible for inhibition of progression of certain types of cells through the cell cycle. The APC protein acts by blocking the Wnt signal-transduction pathway from activating expression of proto-oncogenes, which includes the c-myc gene. With incurring homozygous mutations in the $A P C$ gene, functional APC protein is impaired, and results in an 
incorrect production of Myc, a transcription factor inducing expression of several genes necessary for the G1-S transition the cell cycle. ${ }^{56}$

The APC mutant cells proliferate at a rate that is higher than normal, thus creating polyps. When one of the cells in a polyp undergoes a different activating mutation in the ras gene, its progeny divide even more uncontrollably, forming a larger adenoma. Subsequent mutational loss of a particular chromosomal region (the relevant gene is not yet known), followed by inactivation of the $p 53$ gene, results in chaos due to gradual loss of normal regulation and the consequent formation of a malignant carcinoma. ${ }^{56}$

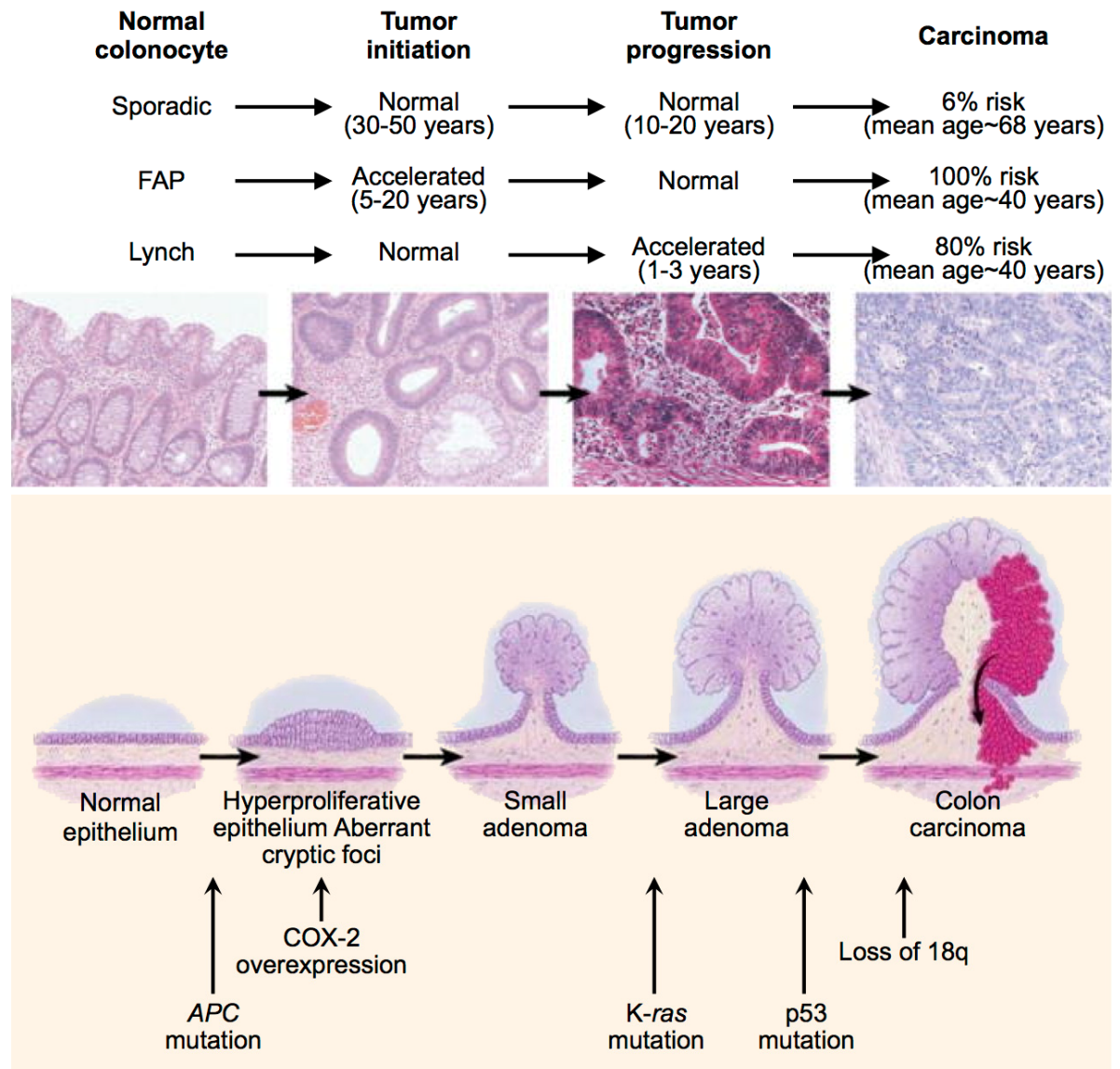

Figure 6. Progression of colon-cancer. ${ }^{57,58}$

Almost half of all human tumors carry mutations in $p 53$, which encodes a transcriptional regulator. DNA from different human colon carcinomas 
generally contains mutations in all these genes-loss-of-function mutations in the tumor suppressors $A P C$ and $p 53$, and an activating (gain-of-function) mutation in the dominant oncogene $K$-ras verifying that multiple mutation in the same cell are needed for the cancer to form. Some of these mutations appear to confer growth advantages at an early stage of tumor development, whereas other mutations promote the later stages, including invasion and metastasis, which are required for the malignant phenotype. Figure 6 shows the sequence of progression of colon cancer together with the suspected gene mutation at each stage.

Molecular markers in colorectal cancer: DNA, RNA and protein biomarkers in stool samples are being widely considered as substitute detection tools for precursor CRC screening. These molecular markers directly reflect the mechanisms of exfoliation of neoplastic cells and secretion of mucus-containing abnormal glycoproteins in CRC. ${ }^{4,59}$ DNA tests from stool for CRC detection are based on methylation ${ }^{60}$, mutation analyses, detection of long DNA ${ }^{61,62}$ and microsatellite instability. ${ }^{63-66}$ Each test makes use of single or multiple DNA markers to optimize performance. ${ }^{4}$

Several marker-based methods are being evaluated for large-scale screening studies. RNA markers, such as $M M P 7$, encoding matrix metalloproteinase-7 also known as matrilysin, and PTGS2, encoding prostaglandin $\mathrm{G} / \mathrm{H}$ synthase 2 , have been assessed in smaller case-control studies, singularly or combining both based on the assumption that they are differentially expressed in cancer and normal tissues. ${ }^{4}$ Protein markers in stool, such as calprotectin, a calcium-binding and zinc-binding neutrophilic cytosolic protein, and carcinoembryonic antigen, are not sensitive enough for adequate detection. ${ }^{4}$ However, another protein, carcino embryonic antigen, is being used to monitor patients with CRC during treatment and subsequent surveillance. ${ }^{68}$ Serum markers are also used for the noninvasive detection of CRC. For plasmabased screening tests, the emphasis is on the detection of methylated DNA, such as methylated septin-9 (SEPT9), where the aberrant methylation of this gene appears to be able to distinguish between normal and cancerous tissues. ${ }^{69}$ The SEPT9 levels in serum are measured by performing plasma sampling, DNA isolation and polymerase chain reaction (PCR) analysis. These experimental techniques are regularly considered in case-control studies. This design may be adequate to establish a proof of concept and selection of the most promising 
combination of markers, however these molecular markers still require further assessment in community screening. Such studies are underway for some tests, such as the SEPT9 test. $^{2}$

Epigenetic biomarkers for colorectal cancer: Apart from DNA, RNA and protein markers in stool, a recent development in early CRC molecular marker is the use of epigenetic markers. Epigenetic modifications, such as changes in DNA methylation, histone modifications, and non-coding RNAs, and play a critical role in carcinogenesis. ${ }^{70}$ Epigenetic aberrations governing TSG inactivation, oncogene activation, and chromosomal instability play a

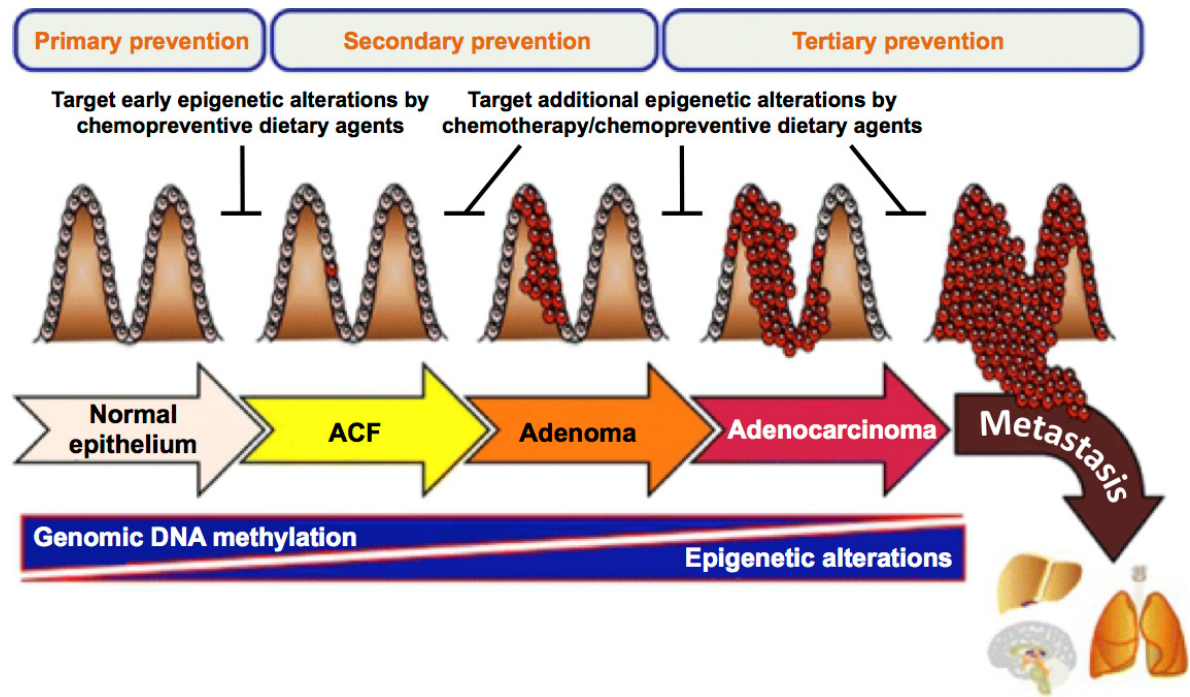

Figure 7. CRC progression as a model for epigenetic alteration cascade and prevention strategies. ${ }^{70}$

fundamental role in tumorigenesis, which includes CRC. Epigenetic events are involved in all critical pathways and the carcinogenesis steps, including tumor initiation, and some events are usually detectable before neoplastic transformation. Epigenetic gene silencing is prevalent in most cancer genes, giving rise to what is known as the epigenomic information of cancer, i.e. 'the hypermethylome ${ }^{71}$ Fig. 7 shows an image representing the high prevalence of hyper-methylation in all types of cancer. ${ }^{71}$ The last ten years have provided an extensive map of the aberrant DNA methylation events occurring in cancer cells, particularly for the hyper-methylated $\mathrm{CpG}$ islands (CPI) of tumor suppressor genes. $^{72,73}$ These data include examples from all classes of human neoplasia and 
have highlighted the existence of a unique profile of hyper-methylated CPIs that defines each tumor type. ${ }^{74,75}$ The emphasis is now to determine the aberrant methylation events that are absent in normal cells, and in developing the techniques that provide reliable, sensitive and fast results to study these potential biomarkers.

In humans, DNA methylation occurs at the $5^{\prime}$ position of the pyrimidine ring of the cytosine residues within the $\mathrm{CpG}$ di-nucleotides through the addition of a methyl moiety to form 5-methylcytosines, as shown in Fig. 8. This process is catalyzed by three DNA methyl-transferases (DNMT1, DNMT3A, and DNMT3B) using the cofactor S-adenosyl-methionine (SAM). Although CpG dinucleotides represent approximately $1 \%$ of the human genome, they are unequally distributed across the genome and are clustered in small DNA stretches. The CPIs are usually present near promoters and exogenic regions. CGI are usually unmethylated in normal differentiated cells, whereas CpGs located in intergenic regions are methylated. ${ }^{71,76}$ In cancer, promoter CGI of numerous TSGs are found to be densely methylated, which results in transcriptional silencing. Interestingly, these epi-mutations may be cancer typespecific and tumor stage-specific. Thus, methylation patterns can be considered as biomarkers for diagnosis, prognosis, as well as prediction and monitoring of therapy response. ${ }^{71,75-77}$<smiles>Nc1cc[nH]c(=O)n1</smiles>

Cytosine<smiles>Cc1c[nH]c(=O)nc1N</smiles>

5-methyl-Cytosine<smiles>Nc1nc(=O)[nH]cc1CO</smiles>

5-hydroxy-methyl-cytosine

Figure 8. Molecular structure of cytosine, 5-methyl-cytosine (5-mC), and 5hydroxy-methyl-cytosine $(5-\mathrm{hmC}){ }^{78}$

Therefore, the identification of these cancer-associated methylation signatures is really critical for cancer prevention purposes. Recent studies show that CRC is strongly associated with aberrant DNA methylation profiles, which has been linked to the origin and progression of the disease. The list of epimutations is growing quickly with the use of developing technologies allowing 
genome studies. To date, a long list of TSG involved in numerous signaling pathways and cellular processes were found frequently methylated in CRC. Figure 9 represents a subset of genes affected by hyper-methylation in CRC. A widespread contribution of DNA methylation in CRC, participates in the disruption of $\beta$-catenin-dependent Wnt signaling pathway, contributing to colorectal tumor development. ${ }^{79,80}$ Moreover, methylation also affects coding and non-coding genes, e.g. microRNA, miRNA, which partakes in loss of tumor suppressor functions. Many of the methylated genes in CRC are being investigated as potential biomarkers for preventive or therapeutic purposes however their methylation prevalence are not universally agreed upon due to inter-study variances depending on genes considered as well as intra-differences within different studies with the same gene.

Recently, epigenetic test technology has advanced by coupling bisulphite modification of DNA with polymerase chain reaction (PCR) sequence readout instruments. ${ }^{81}$ Recently, $\mathrm{CpG}$ island hyper-methylation has been used as a tool to detect cancer cells in several types of biological fluids and tissue biopsies. ${ }^{81,82}$ Since it was first shown that cancer-specific hyper-methylation events could be detected in the sera of individuals with cancer, ${ }^{83}$ a myriad of studies have used this in translational and clinical settings.

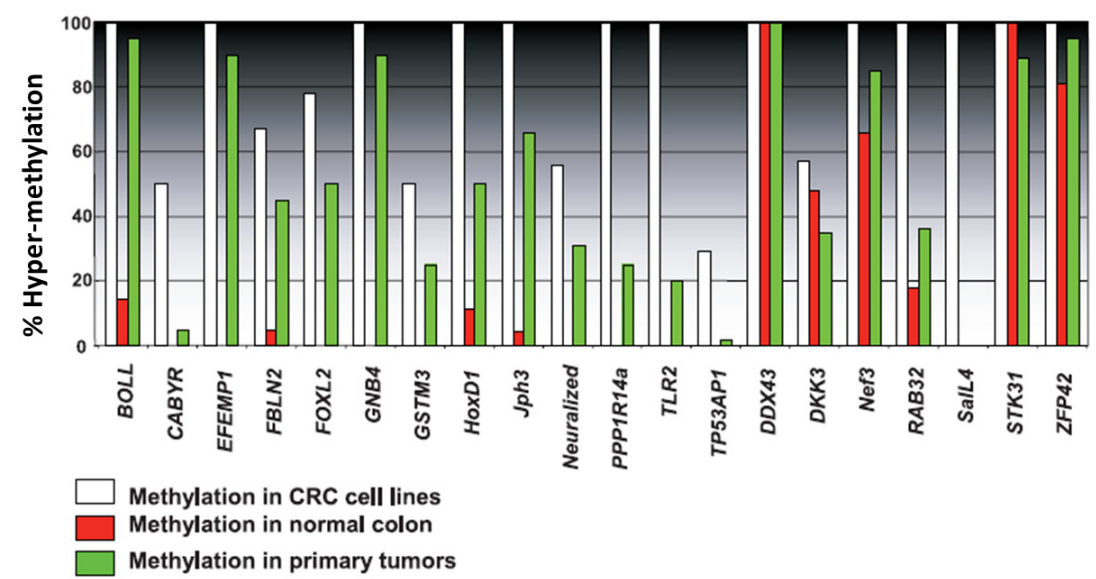

Figure 9. Comparison of hyper-methylation frequencies in CRC cell lines (white columns), normal colon (red columns), or primary tumors (green columns). ${ }^{89}$

Examples include the detection of cancer-specific hyper-methylation events in feces from individuals with colorectal cancer, ${ }^{84}$ urine for bladder cancer 
screening, ${ }^{85}$ or in sputum to predict lung cancer incidence. ${ }^{86}$ Furthermore, new powerful techniques can now detect even minimal amounts of aberrant DNA methylation. ${ }^{87,}{ }^{88}$ Numerous studies have shown that CPI promoter hypermethylation of tumor suppressor genes occurs early in tumorigenesis. However, the presence of aberrant CPI methylation alone does not necessarily indicate an invasive cancer, as premalignant or precursor lesions can also carry these epigenetic signatures. The DNA methylation and histone modification patterns associated with the development and progression of cancer have potential clinical use.

\subsubsection{Epigenetic technology}

We have entered the epigenomics era. Therapeutics, such as Decitabine (5-aza2'-deoxycytidine, MGI Pharma) and Vidaza ${ }^{\mathrm{TM}}$ (5-azacytidine; Pharmion), which are both cytosine analogs that induce DNA hypo-methylation by inhibiting DNA methyltransferase, have been reported to indicate effectiveness for the treatment of myelodysplastic syndromes. ${ }^{90}$ Currently, there is tremendous effort to find ways to exploit the diagnostic and therapeutic implications of DNA methylation abnormalities. ${ }^{91,92}$ Genes, such as APC, SYNE1, GPNMB and MMP2, are normally expressed in the colon, and in CRC are primarily methylated higher than $76 \%$ and mutated. ${ }^{89}$ In recent years, the use of new high-throughput technologies has made it possible to study epigenetic processes at a much broader level than a single gene. The first group of approaches was based on the digestion of genomic DNA with methylation-sensitive restriction enzymes, limiting methylome profiling to the particular sequence motifs bearing specific restriction sites. On the other hand, the employment of genetic and pharmacological unmasking approaches lacked specificity in finding functionally relevant hyper-methylated genes with associations with cancer.

These constraints were overcome using specific antibodies or methylation-binding proteins. Specifically, direct immunoprecipitation of methylated DNA (MeDIP) using a monoclonal antibody against $5 \mathrm{mC}$ has turned out to be a suitable technique for the parallel comparison of two populations in the search for differentially methylated loci. ${ }^{93}$ In addition, coupling this with standard bisulphite genomic sequencing has enabled the identification of a large number of genes with hyper-methylated-CpG islands in colon cancer and other tumor types. ${ }^{93-95}$ The bisulfite treatment of methylated DNA underlies the principle that methylated cytosine remains unchanged, however unmethylated 
cytosine is changed to uracil by deamination. The bisulfite treated DNA is followed with PCR and sequencing for hyper-methylation status investigation. The advent of the bisulphite treatment of DNA, has been a fundamental contribution to cancer epigenetics research. ${ }^{96-98}$ The implementation of bisulphite sequencing in conjunction with genome sequencing, or PCR amplification, methylation-specific PCR (MSP) ${ }^{99}$ allows the examination of DNA methylation of any sequence, and is becoming an invaluable contribution to the field of epigenetics which has been instrumental in key discoveries, such as the epigenetic inactivation of all four genes of the secreted frizzled-related proteins (SFRP) family and other Wnt antagonists in CRC, including DKK-1 and WNT5A, ${ }^{100-102}$ and the silencing of the so-called epigenetic gatekeepers GATA-4 and GATA-5, ${ }^{103}$ and $\beta$-catenin. ${ }^{104}$ The promoter CPI hyper-methylation in different human cancers has been comprehensively analyzed using bisulphite sequencing ${ }^{75}$ which, to date, has been considered the gold standard technique for directly studying DNA methylation and for validating results obtained using other approaches. ${ }^{105}$

The disadvantage of the bisulfite treatment is that it causes DNA degradation, incomplete conversion, low DNA yields, labor intensive and tedious. The bisulfite treatment of DNA analysis is not suitable for large-scale population screening studies of methylation. Hence, protein based methods using antibodies (MeDIP), or methyl-binding domain protein based methods are preferred for methylated DNA analysis. Recently, a cross-platform algorithm for the quantitative analysis of the MeDIP data generated using arrays (MeDIPChip) or genomic-sequencing platforms (MeDIP-seq) has been reported, ${ }^{106}$ but its practical implementation on a routine basis remains to be established. Commercial kits such as Methyl-Miner (Invitrogen), Epimark (New England Biolabs) and Methyl collector (Active motif) involving specific methyl-binding domain (MBD) proteins that can capture symmetrically methylated CpGs in double stranded DNA are available and are being used in favor of the antibody based CpG analysis methods. ${ }^{90}$ Methylated DNA enrichment using these protein based solution assays are then routinely used with conventional bisulfite sequencing or methylation specific PCR for further analysis. ${ }^{90}$ Several new PCR-based methods for detecting DNA methylation using bisulphite-treated DNA are now complementing traditional PCR-based techniques by increasing the analytical sensitivity and providing quantitative information. For instance, 
methylation sensitive melting-curve analysis (MS-MCA) takes advantage of the differential resistance of DNA to melting, depending on the relative GC content. In presence of a fluorescent intercalating chemical, the PCR is conducted and the dye is then integrated in the ds-DNA. A melting analysis performed immediately after the amplification will differentiate the products based on their sequence, which in turn is proportional to the methylation status of the original DNA as two different temperature peaks should be noticed. ${ }^{107}$ An improvement of this technique is the high-resolution melting-curve analysis (MS-HRM). The improvement is with regard to MS-MCA are increased sensitivity, the capacity to adapt it for high-throughput analyses, and better results studying heterogeneously methylated DNA by employing limiting dilutions. ${ }^{108}$ Alternative approaches based on the fundamentals of MSP have also experienced technical advances. The MethyLight technology was developed as the quantitative version of the MSP technique, ${ }^{109,110}$ providing high specificity and sensitivity in the detection of DNA methylation in a high-throughput fashion by using fluorescent DNA methylation-specific probes. The epigenetic landscape for colorectal cancer has expanded. The methylation status can be evaluated in the context of individual genes, genome wide context and sequencing.

All of the methods include an initial step of targeting the 'methylated' DNA signature either chemically, using antibodies, specific proteins, and enzymes prior to the genetic sequence information. For colorectal cancer, several groups have identified methylation biomarkers obtained from fecal DNA or blood samples with informative diagnostic value in cancer detection using these current technologies. ${ }^{111-113}$ It has already been shown that the existing methods have required accuracy and analytical sensitivity, and now await for informative panels of biomarkers to be implemented for specific, straightforward and cost-effective clinical tests.

Epi-genomics for cancer research is emerging with global DNA hypomethylation events in the $1980 \mathrm{~s}$, followed by the CPI hyper-methylation of tumor suppressor genes in the 1990s and the approval of DNA demethylating drugs and histone deactylase inhibitors in the 2000s. DNA methylation at CpG sites in the promoter regions as well DNA non-methylation of CpGs elsewhere both profiles are being sought for epigenetic studies of cancer. Both $\mathrm{CpG}$ methylation based as well as non-CpG-methylation are emergent for the clear 
understanding of role of epigenetic markers. A new epigenetic marker 5-hmC, a second type of DNA methylation, has been identified. 5-hmC was initially described almost 40 years ago, ${ }^{114}$ its rediscovery and the implications as a novel sixth base in the epi-genome has further heightened the excitement in the epigenetics field. From a clinical point of view, the discovery of methylation biomarkers with informative value regarding cancer diagnostics, or predicting prognosis and response to therapy, represents a promising alternative to current invasive procedures or imaging techniques, following time and low-priced protocols. What lies ahead is even more exciting, with the imminent completion of many human cancer epi-genomes that will form the basis of better biomarkers and epigenetic drugs. ${ }^{3,115}$

\subsection{Nanopill: The next generation of CRC screening assay}

Epigenetics based assays have arrived in the clinical setting with the use of the various PCR-based methylation analysis tools, as previously described, and currently the most reliable technique employed in a clinical setting MSP. The original MSP is an inexpensive technique that uses small amounts $(\mu \mathrm{L})$ of bisulphite-converted DNA to provide qualitative information about the methylation status of a given region, and has been widely used in research. In the clinical setting, MSP is being implemented to conduct routine diagnostic tests, such as the case for detecting the MGMT methylated promoter for patient stratification in glioblastomamultiforme. Since patients with inactive MGMT due to aberrant hyper-methylation have been associated with significantly greater long-term benefit from treatment with alkylating therapies than patients with an unmethylated MGMT promoter. ${ }^{116}$ DNA methylation analyses are also of current use to diagnose Prader-Willi syndrome, a disease provoked by an imprinting disorder. ${ }^{117}$

In the case of colorectal cancer, methylation markers have not yet reached the clinic, but are being examined in large prospective trials - SYNE1 and FOXE1, ${ }^{118}$ among others. The power of these sensitive minute amounts of epigenetic biomarkers, such as promoter-hyper-methylation, coupled with mutation detection holds huge promise for next generation CRC screening. There is a need to combine the hyper-methylation detection strategies into simple screening tools without the need for sophisticated and labor-intensive techniques, such as bisulfite analysis, methylation-specific PCR, immune- 
histochemical staining, DNA micro-array and gene expression analysis, for large-scale population screening programs.

Epigenetic abnormalities arise in the earliest steps of colorectal cancer (CRC) development. Aberrant methylation patterns have been identified in preneoplastic lesions including dysplastic aberrant crypt foci, which are considered precursors of colon cancer, and in hyperplastic polyps, which were thought to be benign in nature, and have been proposed to be a risk factor for CRC development as precursor lesions. If the next generation tools can confirm both the state of hyper-methylation and the gene mutation close to the site of infection in vivo, non-invasively such that all possibilities for DNA degradation, false positives, insensitivity is eliminated due to the specificity of these epigenetic markers. The goal of the Nanopill project is just that: perform realtime hyper-methylation and genetic analysis inside the gastrointestinal tract using minute quantities extracted directly from the intestinal tract with the primary importance of specific and early detection colorectal cancer using hypermethylated DNA as the molecular marker.

The Nanopill consists of an automated hyper-methylation detection platform contained in entirely inside a ingestible pill, with similar dimensions as the imaging pill previously described, which administers the epigenetic marker detection in real-time near the source of the of the polyps or lesions. The Nanopill would operate with very small quantities of hypermethylated DNA without the use of PCR amplification, and therefore, free from DNA degradation and un-conversion problems associated with bisulfite sequencing. If successful, the Nanopill will be a quantitative, non-invasive, highly specific molecular epigenetic marker based CRC cancer screening tool. 


\section{References}

1. R. Siegel, D. Naishadham and A. Jemal, CA Cancer Journal for Clinicians, 2012, 62, 10-29.

2. E. J. Kuipers, T. Rösch and M. Bretthauer, Nature Reviews Clinical Oncology, 2013, 10, 130-142.

3. M. Rodríguez-Paredes and M. Esteller, Nature Medicine, 2011, 17, 330-339.

4. L. J. W. Bosch, B. Carvalho, R. J. A. Fijneman, C. R. Jimenez, H. M. Pinedo, M. Van Engeland and G. A. Meijer, Clinical Colorectal Cancer, 2011, 10, 8-23.

5. J. A. Wilschut, J. D. F. Habbema, M. E. Van Leerdam, L. Hol, I. LansdorpVogelaar, E. J. Kuipers and M. Van Ballegooijen, Journal of the National Cancer Institute, 2011, 103, 1741-1751.

6. http://www.aacc.org/publications/cln/ 2011/ march/Pages/Fecal Occult.aspx\#.

7. http://www.intelihealth.com/IH/ihtIH/c/9339/23942.html.

8. L. Hol, M. E. Van Leerdam, M. Van Ballegooijen, A. J. Van Vuuren, H. Van Dekken, J. C. I. Y. Reijerink, A. C. M. Van Der Togt, J. D. F. Habbema and E. J. Kuipers, Gut, 2010, 59, 62-68.

9. L. G. van Rossum, A. F. van Rijn, R. J. Laheij, M. G. van Oijen, P. Fockens, H. H. van Krieken, A. L. Verbeek, J. B. Jansen and E. Dekker, Gastroenterology, 2008, 135, 82-90.

10. M. J. Duffy, L. G. M. Van Rossum, S. T. Van Turenhout, O. Malminiemi, C. Sturgeon, R. Lamerz, A. Nicolini, C. Haglund, L. Holubec, C. G. Fraser and S. P. Halloran, International Journal of Cancer, 2011, 128, 3-11.

11. J. Faivre, V. Dancourt, B. Denis, E. Dorval, C. Piette, P. Perrin, J. M. Bidan, C. Jard, S. Jung, R. Levillain, J. Viguier and J. F. Bretagne, European Journal of Cancer, 2012, 48, 2969-2976.

12. H. Chiba, M. Sekiguchi, T. Ito, Y. Tsuji, K. Ohata, A. Ohno, S. Umezawa, S. Takeuchi, K. Hisatomi, T. Teratani, N. Matsuhashi, H. Endo, M. Inamori and A. Nakajima, Digestive Diseases and Sciences, 2011, 56, 3459-3462.

13. Z. Levi, A. Vilkin and Y. Niv, European Journal of Gastroenterology and Hepatology, 2010, 22, 1431-1434.

14. http://www.mayomedicallaboratories.com/articles/hottopics/transcripts/2011/10fobt/16.html.

15. L. Guittet, E. Guillaume, V. Bouvier and G. Launoy, Gut, 2011, 60, 423-424.

16. L. Guittet, E. Guillaume, R. Levillain, P. Beley, J. Tichet, O. Lantieri and G. 
Launoy, Cancer Epidemiology Biomarkers and Prevention, 2011, 20, 14921501.

17. C. G. Fraser, J. E. Allison, S. P. Halloran and G. P. Young, Journal of the National Cancer Institute, 2012, 104, 810-814.

18. G. Hoff, T. Grotmol, E. Skovlund and M. Bretthauer, BMJ (Clinical research ed.), 2009, 338.

19. W. S. Atkin, R. Edwards, I. Kralj-Hans, K. Wooldrage, A. R. Hart, J. M. Northover, D. M. Parkin, J. Wardle, S. W. Duffy and J. Cuzick, The Lancet, 2010, 375, 1624-1633.

20. N. Segnan, P. Armaroli, L. Bonelli, M. Risio, S. Sciallero, M. Zappa, B. Andreoni, A. Arrigoni, L. Bisanti, C. Casella, C. Crosta, F. Falcini, F. Ferrero, A. Giacomin, O. Giuliani, A. Santarelli, C. B. Visioli, R. Zanetti, W. S. Atkin and C. Senore, Journal of the National Cancer Institute, 2011, 103, 1310-1322.

21. R. E. Schoen, P. F. Pinsky, J. L. Weissfeld, L. A. Yokochi, T. Church, A. O. Laiyemo, R. Bresalier, G. L. Andriole, S. S. Buys, E. D. Crawford, M. N. Fouad, C. Isaacs, C. C. Johnson, D. J. Reding, B. O'Brien, D. M. Carrick, P. Wright, T. L. Riley, M. P. Purdue, G. Izmirlian, B. S. Kramer, A. B. Miller, J. K. Gohagan, P. C. Prorok and C. D. Berg, New England Journal of Medicine, 2012, 366, 2345-2357.

22. http://www.mayoclinic.com/health/medical/IM04057

23. http://www.hopkinsmedicine.org/gastroenterology_hepatology/clinical_services/basic_en doscopy/flexible_sigmoidoscopy.html

24. R. Valori, J. F. Rey, W. S. Atkin, M. Bretthauer, C. Senore, G. Hoff, E. J. Kuipers, L. Altenhofen, R. Lambert and G. Minoli, Endoscopy, 2012, 44, SE88-SE105.

25. http://www.tabletprep.com/colonoscopy/index.aspx.

26. M. F. Kaminski, J. Regula, E. Kraszewska, M. Polkowski, U. Wojciechowska, J. Didkowska, M. Zwierko, M. Rupinski, M. P. Nowacki and E. Butruk, New England Journal of Medicine, 2010, 362, 1795-1803.

27. C. Stock, P. Ihle, I. Schubert and H. Brenner, Endoscopy, 2011, 43, 771-779.

28. S. S. Rogal, P. F. Pinsky and R. E. Schoen, Clinical Gastroenterology and Hepatology, 2013, 11, 73-78.

29. B. Bressler, L. F. Paszat, Z. Chen, D. M. Rothwell, C. Vinden and L. Rabeneck, Gastroenterology, 2007, 132, 96-102.

30. B. Lebwohl, F. Kastrinos, M. Glick, A. J. Rosenbaum, T. Wang and A. I. 
Neugut, Gastrointestinal Endoscopy, 2011, 73, 1207-1214.

31. D. Armstrong, A. Barkun, R. Bridges, R. Carter, C. De Gara, C. Dubé, R. Enns, R. Hollingworth, D. MacIntosh, M. Borgaonkar, S. Forget, G. Leontiadis, J. Meddings, P. Cotton, E. J. Kuipers and R. Valori, Canadian Journal of Gastroenterology, 2012, 26, 17-31.

32. R. Jover, M. Herráiz, O. Alarcón, E. Brullet, L. Bujanda, M. Bustamante, R. Campo, R. Carreño, A. Castells, J. Cubiella, P. García-Iglesias, A. J. Hervás, P. Menchén, A. Ono, A. Panadés, A. Parra-Blanco, M. Pellisé, M. Ponce, E. Quintero, J. M. Reñé, A. Sánchez Del Río, A. Seoane, A. Serradesanferm, A. Soriano Izquierdo and E. Vázquez Sequeiros, Endoscopy, 2012, 44, 444451.

33. D. A. Lieberman, D. K. Rex, S. J. Winawer, F. M. Giardiello, D. A. Johnson and T. R. Levin, Gastroenterology, 2012, 143, 844-857.

34. E. M. Stoop, M. C. de Haan, T. R. de Wijkerslooth, P. M. Bossuyt, M. van Ballegooijen, C. Y. Nio, M. J. van de Vijver, K. Biermann, M. Thomeer, M. E. van Leerdam, P. Fockens, J. Stoker, E. J. Kuipers and E. Dekker, The Lancet Oncology, 2012, 13, 55-64.

35. A. Graser, P. Stieber, D. Nagel, C. Schäfer, D. Horst, C. R. Becker, K. Nikolaou, A. Lottes, S. Geisbüsch, H. Kramer, A. C. Wagner, H. Diepolder, J. Schirra, H. J. Roth, D. Seidel, B. Göke, M. F. Reiser and F. T. Kolligs, Gut, 2009, 58, 241-248.

36. D. H. Kim, P. J. Pickhardt, A. J. Taylor, W. K. Leung, T. C. Winter, J. L. Hinshaw, D. V. Gopal, M. Reichelderfer, R. H. Hsu and P. R. Pfau, New England Journal of Medicine, 2007, 357, 1403-1412.

37. C. D. Johnson, M. H. Chen, A. Y. Toledano, J. P. Heiken, A. Dachman, M.

D. Kuo, C. O. Menias, B. Siewert, J. I. Cheema, R. G. Obregon, J. L. Fidler, P. Zimmerman, K. M. Horton, K. Coakley, R. B. Iyer, A. K. Hara, R. A. Halvorsen Jr, G. Casola, J. Yee, B. A. Herman, L. J. Burgart and P. J. Limburg, New England Journal of Medicine, 2008, 359, 1207-1217.

38. D. H. Kim, B. D. Pooler, J. M. Weiss and P. J. Pickhardt, European Radiology, 2012, 22, 1488-1494.

39. M. C. De Haan, C. Y. Nio, M. Thomeer, A. H. De Vries, P. M. Bossuyt, E. J. Kuipers, E. Dekker and J. Stoker, Radiology, 2012, 264, 771-778.

40. D. J. Vanness, A. B. Knudsen, I. Lansdorp-Vogelaar, C. M. Rutter, I. F. Gareen, B. A. Herman, K. M. Kuntz, A. G. Zauber, M. Van Ballegooijen, E. 
J. Feuer, M. H. Chen and C. D. Johnson, Radiology, 2011, 261, 487-498.

41. L. C. Richardson, E. Tai, S. H. Rim, D. Joseph and M. Plescia, Morbidity and Mortality Weekly Report, 2011, 60, 884-889.

42. B. K. Edwards, E. Ward, B. A. Kohler, C. Eheman, A. G. Zauber, R. N. Anderson, A. Jemal, M. J. Schymura, I. Lansdorp-Vogelaar, L. C. Seeff, M. van Ballegooijen, S. L. Goede and L. A. G. Ries, Cancer, 2010, 116, 544573.

43. C. J. Kahi, J. C. Anderson and D. K. Rex, Gastrointestinal Endoscopy, 2013, 77, 335-350.

44. M. H. Liedenbaum, A. F. Van Rijn, A. H. De Vries, H. M. Dekker, M. Thomeer, C. J. Van Marrewijk, L. Hol, M. G. W. Dijkgraaf, P. Fockens, P. M. M. Bossuyt, E. Dekker and J. Stoker, Gut, 2009, 58, 1242-1249.

45. J. A. Wilschut, E. W. Steyerberg, M. E. Van Leerdam, I. Lansdorp-Vogelaar, J. D. F. Habbema and M. Van Ballegooijen, Cancer, 2011, 117, 4166-4174.

46. J. A. Wilschut, L. Hol, E. Dekker, J. B. Jansen, M. E. Van Leerdam, I. Lansdorp-Vogelaar, E. J. Kuipers, J. D. F. Habbema and M. Van Ballegooijen, Gastroenterology, 2011, 141, 1648-1655.

47. K. M. Kuntz, I. Lansdorp-Vogelaar, C. M. Rutter, A. B. Knudsen, M. Van Ballegooijen, J. E. Savarino, E. J. Feuer and A. G. Zauber, Medical Decision Making, 2011, 31, 530-539.

48. T. R. De Wijkerslooth, E. M. Stoop, P. M. Bossuyt, E. M. H. MathusVliegen, J. Dees, K. M. A. J. Tytgat, M. E. Van Leerdam, P. Fockens, E. J. Kuipers and E. Dekker, Gut, 2012, 61, 1426-1434.

49. A. M. Leufkens, D. C. Demarco, A. Rastogi, P. A. Akerman, K. Azzouzi, R. I. Rothstein, F. P. Vleggaar, A. Repici, G. Rando, P. I. Okolo, O. Dewit, A. Ignjatovic, E. Odstrcil, J. East, P. H. Deprez, B. P. Saunders, A. N. Kalloo, B. Creel, V. Singh, A. M. Lennon and P. D. Siersema, Gastrointestinal Endoscopy, 2011, 73, 480-489.

50. F. W. Leung, A. Amato, C. Ell, S. Friedland, J. O. Harker, Y. H. Hsieh, J. W. Leung, S. K. Mann, S. Paggi, J. Pohl, F. Radaelli, F. C. Ramirez, R. SiaoSalera and V. Terruzzi, Gastrointestinal Endoscopy, 2012, 76, 657-666.

51. A. Adler, A. Aminalai, J. Aschenbeck, R. Drossel, M. Mayr, M. Scheel, A. Schröder, T. Yenerim, B. Wiedenmann, U. Gauger, S. Roll and T. Rösch, Clinical Gastroenterology and Hepatology, 2012, 10, 155-159.

52. R. Eliakim, Gastroenterology, 2010, 139, 1468-1480. 
53. A. Van Gossum, M. M. Navas, I. Fernandez-Urien, C. Carretero, G. Gay, M. Delvaux, M. G. Lapalus, T. Ponchon, H. Neuhaus, M. Philipper, G. Costamagna, M. E. Riccioni, C. Spada, L. Petruzziello, C. Fraser, A. Postgate, A. Fitzpatrick, F. Hagenmuller, M. Keuchel, N. Schoofs and J. Devière, New England Journal of Medicine, 2009, 361, 264-270.

54. C. Spada, C. Hassan, M. Munoz-Navas, H. Neuhaus, J. Deviere, P. Fockens, E. Coron, G. Gay, E. Toth, M. E. Riccioni, C. Carretero, J. P. Charton, A. Van Gossum, C. A. Wientjes, S. Sacher-Huvelin, M. Delvaux, A. Nemeth, L. Petruzziello, C. P. De Frias, R. Mayershofer, L. Aminejab, E. Dekker, J. P. Galmiche, M. Frederic, G. W. Johansson, P. Cesaro and G. Costamagna, Gastrointestinal Endoscopy, 2011, 74, 581-589.

55. C. Spada, C. Hassan, R. Marmo, L. Petruzziello, M. E. Riccioni, A. Zullo, P. Cesaro, J. Pilz and G. Costamagna, Clinical Gastroenterology and Hepatology, 2010, 8, 516-522.

56. H. Lodish, A. Berk, C. A. Kaiser, M. Krieger, M. P. Scott, A. Bretscher, H. Ploegh and P. Matsudaira, Molecular Cell Biology, W. H. Freeman and Company, 2008.

57. W. M. Grady and J. M. Carethers, Gastroenterology, 2008, 135, 1079-1099.

58. http://abnormalfacies.wordpress.com/2011/04/07/how-colonoscopies-save-lives/

59. N. K. Osborn and D. A. Ahlquist, Gastroenterology, 2005, 128, 192-206.

60. S. C. Gckner, M. Dhir, M. Y. Joo, K. E. McGarvey, L. Van Neste, J. Louwagie, T. A. Chan, W. Kleeberger, A. P. De Bru $\tilde{A}^{-}$ne, K. M. Smits, C. A. J. Khalid-de Bakker, D. M. A. E. Jonkers, R. W. Stockbrgger, G. A. Meijer, F. A. Oort, C. Iacobuzio-Donahue, K. Bierau, J. G. Herman, S. B. Baylin, M. Van Engeland, K. E. Schuebel and N. Ahuja, Cancer Research, 2009, 69, 4691-4699.

61. H. Zou, J. Harrington, R. L. Rego and D. A. Ahlquist, Clinical Chemistry, 2007, 53, 1646-1651.

62. H. Zou, J. J. Harrington, K. K. Klatt and D. A. Ahlquist, Cancer Epidemiology Biomarkers and Prevention, 2006, 15, 1115-1119.

63. M. Esteller, R. Levine, S. B. Baylin, L. H. Ellenson and J. G. Herman, Oncogene, 1998, 17, 2413-2417.

64. A. S. Fleisher, M. Esteller, N. Harpaz, A. Leytin, A. Rashid, Y. Xu, J. Liang, O. C. Stine, J. Yin, T. T. Zou, J. M. Abraham, D. Kong, K. T. Wilson, S. P. James, J. G. Herman and S. J. Meltzer, Cancer Research, 2000, 60, 4864- 
4868.

65. Y. H. Kim, Z. Petko, S. Dzieciatkowski, L. Lin, M. Ghiassi, S. Stain, W. C. Chapman, M. K. Washington, J. Willis, S. D. Markowitz and W. M. Grady, Genes Chromosomes and Cancer, 2006, 45, 781-789.

66. P. Laiho, V. Launonen, P. Lahermo, M. Esteller, M. Guo, J. G. Herman, J. P. Mecklin, H. Järvinen, P. Sistonen, K. M. Kim, D. Shibata, R. S. Houlston and L. A. Aaltonen, Cancer Research, 2002, 62, 1166-1170.

67. D. A. Ahlquist, H. Zou, M. Domanico, D. W. Mahoney, T. C. Yab, W. R. Taylor, M. L. Butz, S. N. Thibodeau, L. Rabeneck, L. F. Paszat, K. W. Kinzler, B. Vogelstein, N. C. Bjerregaard, S. Laurberg, H. T. Sørensen, B. M. Berger and G. P. Lidgard, Gastroenterology, 2012, 142, 248-256.

68. N. Wild, H. Andres, W. Rollinger, F. Krause, P. Dilba, M. Tacke and J. Karl, Clinical Cancer Research, 2010, 16, 6111-6121.

69. C. Lofton-Day, F. Model, T. DeVos, R. Tetzner, J. Distler, M. Schuster, X. Song, R. Lesche, V. Liebenberg, M. Ebert, B. Molnar, R. Grützmann, C. Pilarsky and A. Sledziewski, Clinical Chemistry, 2008, 54, 414-423.

70. M. Schnekenburger and M. Diederich, Current Colorectal Cancer Reports, 2012, 8, 66-81.

71. M. Esteller, Human Molecular Genetics, 2007, 16, R50-R59.

72. M. Esteller, New England Journal of Medicine, 2008, 358, 1148-1159.

73. J. G. Herman and S. B. Baylin, New England Journal of Medicine, 2003, 349, 2042-2054.

74. J. F. Costello, M. C. Frühwald, D. J. Smiraglia, L. J. Rush, G. P. Robertson, X. Gao, F. A. Wright, J. D. Feramisco, P. Peltomäki, J. C. Lang, D. E. Schuller, L. Yu, C. D. Bloomfield, M. A. Caligiuri, A. Yates, R. Nishikawa, H. J. Su Huang, N. J. Petrelli, X. Zhang, M. S. O'Dorisio, W. A. Held, W. K. Cavenee and C. Plass, Nature Genetics, 2000, 24, 132-138.

75. M. Esteller, P. G. Corn, S. B. Baylin and J. G. Herman, Cancer Research, 2001, 61, 3225-3229.

76. R. Taby and J. P. Issa, CA Cancer J Clin, 2010, 60, 376-392.

77. C. Florean, M. Schnekenburger, C. Grandjenette, M. Dicato and M. Diederich, Epigenomics, 2011, 3, 581-609.

78. J. I. Martín-Subero, E. Ballestar, M. Esteller and R. Siebert, Leukemia, 2006, 20, 1658-1660.

79. L. Migliore, F. Migheli, R. Spisni and F. Copped, Journal of Biomedicine 
and Biotechnology, 2011, Article ID 792362, 19 pages.

80. E. R. Fearon, Annual Review of Pathology: Mechanisms of Disease, 2011, 6, 479-507.

81. P. W. Laird, Nature Reviews Cancer, 2003, 3, 253-266.

82. N. Shivapurkar and A. F. Gazdar, Current Molecular Medicine, 2010, 10, 123-132.

83. M. Esteller, M. Sanchez-Cespedes, R. Resell, D. Sidransky, S. B. Baylin and J. G. Herman, Cancer Research, 1999, 59, 67-70.

84. W. D. Chen, Z. J. Han, J. Skoletsky, J. Olson, J. Sah, L. Myeroff, P. Platzer, S. Lu, D. Dawson, J. Willis, T. P. Pretlow, J. Lutterbaugh, L. Kasturi, J. K. V. Willson, J. S. Rao, A. Shuber and S. D. Markowitz, Journal of the National Cancer Institute, 2005, 97, 1124-1132.

85. M. O. Hoque, S. Begum, O. Topaloglu, A. Chatterjee, E. Rosenbaum, W. Van Criekinge, W. H. Westra, M. Schoenberg, M. Zahurak, S. N. Goodman and D. Sidransky, Journal of the National Cancer Institute, 2006, 98, 9961004.

86. S. A. Belinsky, K. C. Liechty, F. D. Gentry, H. J. Wolf, J. Rogers, K. Vu, J. Haney, T. C. Kennedy, F. R. Hirsch, Y. Miller, W. A. Franklin, J. G. Herman, S. B. Baylin, P. A. Bunn and T. Byers, Cancer Research, 2006, 66, 33383344.

87. V. J. Bailey, H. Easwaran, Y. Zhang, E. Griffiths, S. A. Belinsky, J. G. Herman, S. B. Baylin, H. E. Carraway and T. H. Wang, Genome Research, 2009, 19, 1455-1461.

88. M. Li, W. D. Chen, N. Papadopoulos, S. N. Goodman, N. C. Bjerregaard, S. Laurberg, B. Levin, H. Juhl, N. Arber, H. Moinova, K. Durkee, K. Schmidt, Y. He, F. Diehl, V. E. Velculescu, S. Zhou, L. A. Diaz, K. W. Kinzler, S. D. Markowitz and B. Vogelstein, Nature Biotechnology, 2009, 27, 858-863.

89. K. E. Schuebel, W. Chen, L. Cope, S. C. GlÃ $₫$ ckner, H. Suzuki, J. M. Yi, T. A. Chan, L. Van Neste, W. Van Criekinge, S. Van Den Bosch, M. Van Engeland, A. H. Ting, K. Jair, W. Yu, M. Toyota, K. Imai, N. Ahuja, J. G. Herman and S. B. Baylin, PLoS Genetics, 2007, 3, 1709-1723.

90. L. G. Acevedo, A. Sanz and M. A. Jelinek, Epigenomics, 2011, 3, 93-101.

91. M. Esteller, Journal of Pathology, 2005, 205, 172-180.

92. T. Kalebic, Annals of the New York Academy of Sciences, 2003, vol. 983, pp. 278-285. 
93. M. Weber, J. J. Davies, D. Wittig, E. J. Oakeley, M. Haase, W. L. Lam and D. Schubeler, Nature Genetics, 2005, 37, 853-862.

94. F. V. Jacinto, E. Ballestar, S. Ropero and M. Esteller, Cancer Research, 2007, 67, 11481-11486.

95. I. Keshet, Y. Schlesinger, S. Farkash, E. Rand, M. Hecht, E. Segal, E. Pikarski, R. A. Young, A. Niveleau, H. Cedar and I. Simon, Nature Genetics, 2006, 38, 149-153.

96. M. Esteller, Nature Reviews Genetics, 2007, 8, 286-298.

97. M. Frommer, L. E. McDonald, D. S. Millar, C. M. Collis, F. Watt, G. W. Grigg, P. L. Molloy and C. L. Paul, Proceedings of the National Academy of Sciences of the United States of America, 1992, 89, 1827-1831.

98. S. J. Clark, J. Harrison, C. L. Paul and M. Frommer, Nucleic Acids Research, 1994, 22, 2990-2997.

99. J. G. Herman, J. R. Graff, S. Myöhänen, B. D. Nelkin and S. B. Baylin, Proceedings of the National Academy of Sciences of the United States of America, 1996, 93, 9821-9826.

100. H. Suzuki, D. N. Watkins, K. W. Jair, K. E. Schuebel, S. D. Markowitz, W. D. Chen, T. P. Pretlow, B. Yang, Y. Akiyama, M. Van Engeland, M. Toyota, T. Tokino, Y. Hinoda, K. Imai, J. G. Herman and S. B. Baylin, Nature Genetics, 2004, 36, 417-422.

101. O. Aguilera, M. F. Fraga, E. Ballestar, M. F. Paz, M. Herranz, J. Espada, J. M. Garcia, A. Munoz, M. Esteller and J. M. Gonzalez-Sancho, Oncogene, 2006, 25, 4116-4121.

102. J. Ying, H. Li, J. Yu, K. M. Ng, F. F. Poon, S. C. C. Wong, A. T. C. Chan, J. J. Y. Sung and Q. Tao, Clinical Cancer Research, 2008, 14, 55-61.

103. Y. Akiyama, N. Watkins, H. Suzuki, K. W. Jair, M. Van Engeland, M. Esteller, H. Sakai, C. Y. Ren, Y. Yuasa, J. G. Herman and S. B. Baylin, Molecular and Cellular Biology, 2003, 23, 8429-8439.

104. S. B. Baylin and J. E. Ohm, Nature Reviews Cancer, 2006, 6, 107-116.

105. F. Model, N. Osborn, D. Ahlquist, R. Gruetzmann, B. Molnar, F. Sipos, O. Galamb, C. Pilarsky, H. D. Saeger, Z. Tulassay, K. Hale, S. Mooney, J. Lograsso, P. Adorjan, R. Lesche, A. Dessauer, J. Kleiber, B. Porstmann, A. Sledziewski and C. Lofton-Day, Molecular Cancer Research, 2007, 5, 153163.

106. T. A. Down, V. K. Rakyan, D. J. Turner, P. Flicek, H. Li, E. Kulesha, S. 
Gräf, N. Johnson, J. Herrero, E. M. Tomazou, N. P. Thorne, L. Bäckdahl, M. Herberth, K. L. Howe, D. K. Jackson, M. M. Miretti, J. C. Marioni, E. Birney, T. J. P. Hubbard, R. Durbin, S. Tavaré and S. Beck, Nature Biotechnology, 2008, 26, 779-785.

107. J. Worm, A. Aggerholm and P. Guldberg, Clinical Chemistry, 2001, 47, 1183-1189.

108. I. L. Candiloro, T. Mikeska, P. Hokland and A. Dobrovic, Epigenetics \& Chromatin 2008, 1-7.

109. C. A. Eads, K. D. Danenberg, K. Kawakami, L. B. Saltz, C. Blake, D. Shibata, P. V. Danenberg and P. W. Laird, Nucleic Acids Research, 2000, 28.

110. B. N. Trinh, T. I. Long and P. W. Laird, Methods, 2001, 25, 456-462.

111. T. Kawasaki, M. Ohnishi, K. Nosho, Y. Suemoto, G. J. Kirkner, J. A. Meyerhardt, C. S. Fuchs and S. Ogino, Mod Pathol, 2008, 21, 245-255.

112. M. Oberwalder, M. Zitt, C. Wöntner, H. Fiegl, G. Goebel, O. Köhle, G. Mühlmann, D. Öfner, R. Margreiter and H. M. Müller, International Journal of Colorectal Disease, 2008, 23, 15-19.

113. D. R. Wang and D. Tang, World Journal of Gastroenterology, 2008, 14, 524-531.

114. N. W. Penn, R. Suwalski, C. O'Riley, K. Bojanowski and R. Yura, Biochemical Journal, 1972, 126, 781-790.

115. M. Esteller, Scientist, 2011, 25, 34.

116. R. Martinez, G. Schackert, R. Yaya-Tur, I. Rojas-Marcos, J. G. Herman and M. Esteller, Journal of Neuro-Oncology, 2007, 83, 91-93.

117. F. J. Carmona and M. Esteller, Mutation Research - Fundamental and Molecular Mechanisms of Mutagenesis, 2010, 693, 53-60.

118. P. Mokarram, K. Kumar, H. Brim, F. Naghibalhossaini, M. Saberi-firoozi, M. Nouraie, R. Green, E. Lee, D. T. Smoot and H. Ashktorab, PLoS ONE, 2009, 4, e7012. 


\section{Chapter 3}

\section{Nanopill: Miniature automated diagnostic system}

Cancer is a major cause of death in men and women around the globe and exists in many forms. The successful treatment of cancer treatment relies on its early detection; in many cases, a survival rate near $90 \%$ is possible if the tumor is detected at an early state of progression, or Stage I. Therefore, it is imperative that new sensitive detection technologies be introduced that can detect a small number of biomarker copies, both sensitively and specifically, near the origin of the tumor. In this chapter, an automated miniature colon cancer diagnosis system concept is presented, called the Nanopill, which is in development in collaboration with Dr. Bob Pinedo, an Oncologist formerly with Vrije Universiteit Medical Center, Amsterdam. 


\subsection{Introduction}

A major public health problem around the world is the spread of cancer. ${ }^{2}$ Cancer exists in many distinct forms, such as cancer in the colon, lung, prostate, breast, pancreas, ovary, and blood. Cancer is defined as abnormal and uncontrolled cell growth due to an accumulation of specific genetic and epigenetic defects, both environmental and hereditary in origin. ${ }^{3-5}$ Most cancers are measured in four stages: Stage I is early stage where the cancer is contained in the organ of origin; Stage II and III, the cancer has metastasized into surrounding tissue and organs; and Stage IV is late stage where the malignancy has spread into the major organs. When a tumor is discovered in stage I, the survival rate is nearly $90 \%$ because treatment typically consists of surgery and mild chemotherapy. When the tumor is discovered in Stage IV, the survival rate drops to about $10 \%{ }^{4,6,7}$ The importance of early warning disease detection is well-understood and new methods that can simplify the testing procedure while maintaining high levels of sensitivity, specificity and minimal false-positive results are needed. It is imperative that new detection technologies with high specificity and sensitivity be available to detect small number of biomarker copies near the origin of the tumor.

Colorectal cancer is one of the most common malignancies that will be developed by at least $13 \%$ of the western population, and is considered one of the leading causes of cancer related deaths. ${ }^{5}$ The fecal occult blood test is the most common screening method for colorectal cancer but is considered to have poor sensitivity. Other screening techniques include endoscopic procedures, such as flexible sigmoidoscopy and colonoscopy. These procedures are considered sensitive and specific for identifying cancerous regions of the colon, however, they are invasive and costly, and therefore, not widely administered.

More recently, a new screening technique for colon cancer, as well as other types of cancers, is the detection of aberrant methylation, or hypermethylation of normally un-methylated $\mathrm{CpG}$ islands of DNA in specific gene sequences. ${ }^{8,9}$ A plethora of methods have been developed for mapping methylated DNA regions by combining bisulfite treatment with a methylation specific polymerase chain reaction, ${ }^{10,11}$ southern blot hybridization assays, ${ }^{12}$ and methylation specific restriction enzymes, ${ }^{13}$ for both local and global genome wide methylation analysis. ${ }^{14}$ More recently, specific DNA methylation detection using microarray formats for high throughput screening of patient samples has 
been reported. ${ }^{15}$ One important disadvantage is the requirement of stool collection, subsequent freezing, and transportation to a laboratory where the assay is performed.

Quite a few smart pills integrating electronic recording with body parameter determination is already available commercially as summarized in Table 1 below.

Table 1. Summary of commercially available ingestible smart-pills.

\begin{tabular}{|c|c|l|}
\hline Name & Affiliation & \multicolumn{1}{|c|}{ Purpose } \\
\hline Smartpill & Given Imaging & $\begin{array}{l}\text { SmartPill GI Monitoring System: It can measure } \\
\text { pressure, pH and temperature while travelling } \\
\text { through the gastrointestinal (GI) tract to assess GI } \\
\text { motility. The SmartPill motility monitoring test can } \\
\text { be performed at a clinic or physician's office to } \\
\text { evaluate motility disorders like gastroparesis a } \\
\text { condition in which the contents of the stomach } \\
\text { empty too slowly) and chronic constipation. }\end{array}$ \\
\hline CorTemp & HQ Inc & $\begin{array}{l}\text { Ingestible core body thermometer pill and the RF } \\
\text { compatible CorTemp miniaturized data recorder }\end{array}$ \\
\hline e-Pill & Proteus Digital & $\begin{array}{l}\text { FDA has cleared this unique tiny ingestible sensor. } \\
\text { The sensor will transmit information about the user } \\
\text { vital signs as well as how well they handle their } \\
\text { prescribed medication regimen and monitored } \\
\text { wirelessly and transmitted to a patch on the user's } \\
\text { body and then to an application on the users mobile } \\
\text { device. }\end{array}$ \\
\hline Battery driven Pill \\
cam COLON
\end{tabular}


In this chapter, an automated miniature colon cancer diagnosis system is presented, called the Nanopill, which has been developed in collaboration with Dr. Bob Pinedo, an Oncologist formerly with Vrije Universiteit Medical Center, Amsterdam. ${ }^{1}$ The many different techniques reported over the last twenty years to detect and map DNA methylation of serum and fecal samples are synthesized and a miniature automated diagnostic system concept is presented. The new miniature diagnostic system, called the Nanopill, is an automated diagnostic pill, small enough to be safely ingested by a patient and would automatically collect and analyze intestinal fluid for a priori known colon cancer biomarker, shown in the cartoon in Fig. 1.

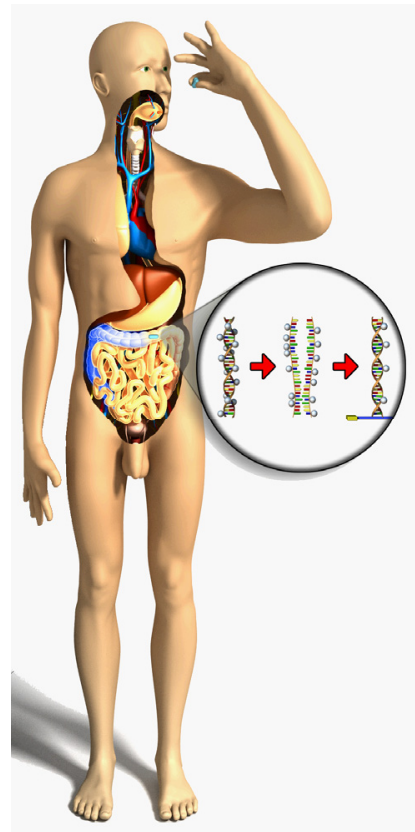

Figure 1. Nanopill automated diagnostic system.

The Nanopill system would notify the patient and treating physician by wireless transmission to a smart-phone, for example. An important advantage of an assay to be performed in the bowel is that the sensitivity is increased as compared to the assay, which requires handling of the stools in the laboratory. This is mainly because the DNA gets degraded in stool, if not frozen and reached fast enough to the laboratory. 


\subsection{Nanopill automated diagnostic bioassay}

The basic operation of the Nanopill system, as shown in the four panels of Fig. 2, begins with the patient swallowing the pill (Fig. 2(a)). When the Nanopill reaches the lower intestine, the pill begins to collect the intestinal fluid. The sample fluid is purified and free-floating hyper-methylated DNA (hm-DNA) is isolated and pre-concentrated, and subsequently transported to the detection stage (Fig. 2(b)). When a positive detection event occurs, the patient is notified by a wireless transmission (Fig. 2(c)) to the treating physician and the patient (Fig. 2(d)).

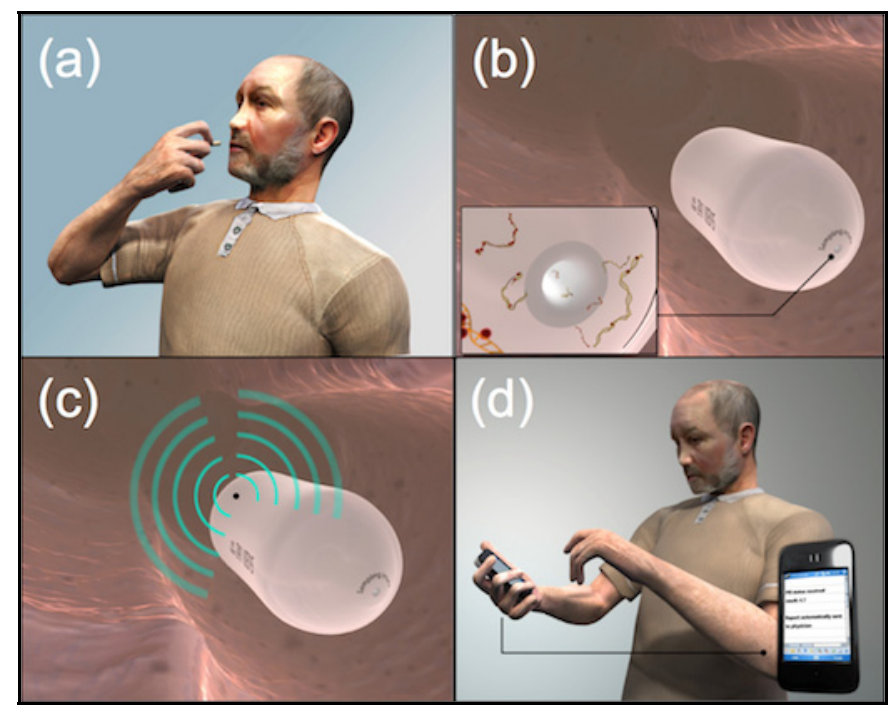

Figure 2. Conceptual Nanopill automated diagnostic assay. (a) Ingestion (b) Transport through intestine sampling intestinal fluid and automated sample processing and marker detection (c) assay results transmitted wirelessly (d) results read with conventional smart-phone.

This type of early warning disease detection can revolutionize methylated-related cancer screening and if successful, will drastically reduce cancer-causing fatalities. The concept Nanopill diagnostic system consists of many interacting components, but can be described best by outlining the five main system components: 1. Sample extraction, purification and preconcentration; 2. DNA detection; 3. Electronics; 4. Wireless signal generation; and 5. Electrical power supply. The system component design and implementation have been done in the BIOS Group and the MESA+ Institute for Nanotechnology. The molecular assay has been developed and implemented in 
close collaboration with Dr. Bob Pinedo. The detailed Nanopill system and components will be described in more detail.

\subsection{Nanopill system and components}

The five primary Nanopill system concepts are shown schematically in Fig. 3. The main functions of the Nanopill include: hm-DNA extraction, purification and pre-concentration, hm-DNA detection, electronics, wireless signal transmission, and electrical battery power supply. The Nanopill system begins with the patient swallowing the pill. When the Nanopill reaches the lower intestine, the pill begins to collect intestinal fluid. The sample fluid is mixed with a pre-stored salt (orange container, Fig. 3) solution that purifies the hmDNA, which is subsequently extracted with a miniature extraction column prepared with proteins that specifically bind to the hyper-methylated regions of DNA.

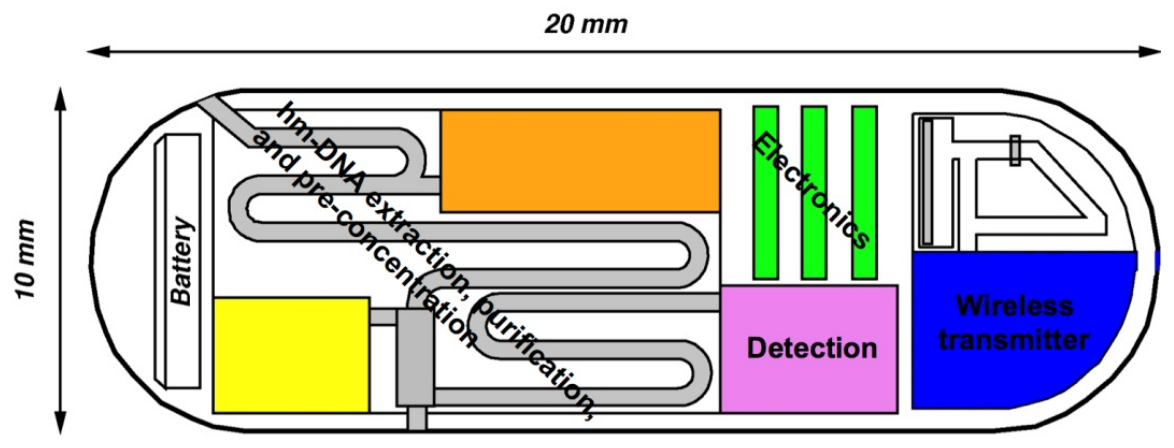

Figure 3. Nanopill system components.

The captured hm-DNA molecules are then eluted from the column with a prestored buffer (yellow container, Fig. 3) and transported to the detection stage, which contains biosensors that have probe molecules attached to the sensor surface with a genomic sequence specific for the epigenetic marker tagged DNA for colon cancer. The complete operation of the Nanopill is automated and controlled by the "brains" of the system, the electronic control chip. When a positive detection event occurs, the integrated electronics commands that a wireless signal is transmitted to the treating physician and patient. All components of the Nanopill can be realized with conventional microtechnology fabrication methods. 


\subsubsection{Sample extraction and purification.}

The intestinal fluid is collected from the external environment of the ingested Nanopill. The collection of the intestinal fluid is to be performed with an integrated pump system capable of withdrawing an adequate amount $(\sim 1 \mathrm{ml})$ of intestinal fluid and mixing with a wash buffer solution. The microfluidic system contains a miniature silica pillar array with high surface area and functionalized with specific

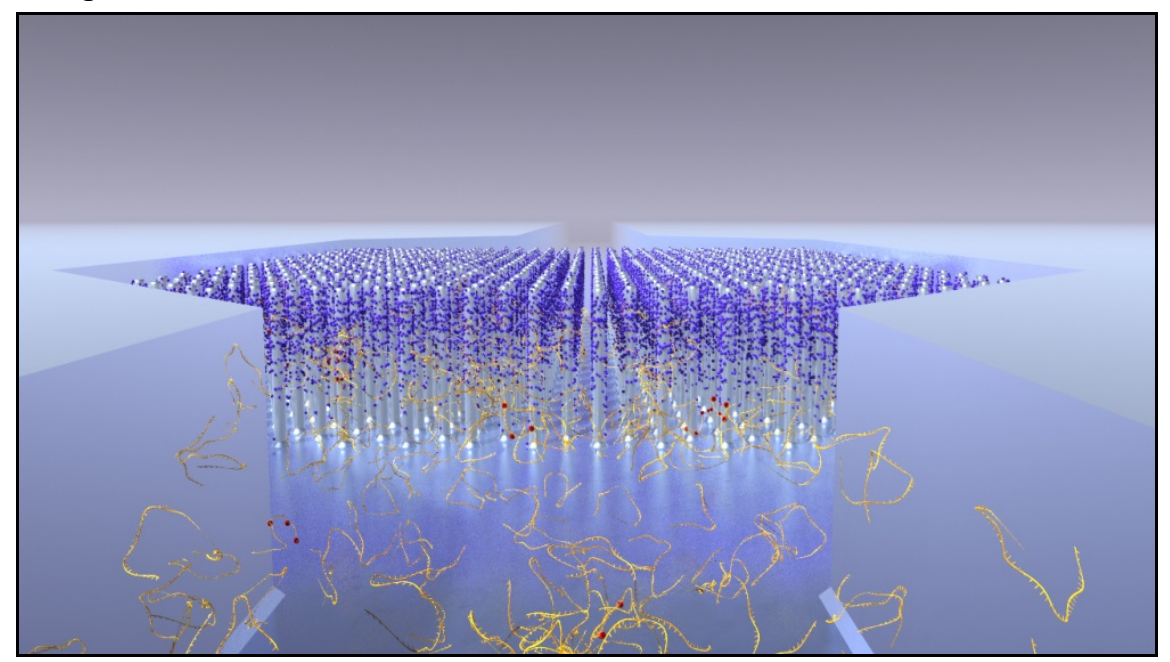

Figure 4. Cartoon illustration of the microfluidic hm-DNA capture process.

methyl-binding-domain (MBD) proteins to capture the hm-DNA directly from the purified sample. The captured hm-DNA is then released by flowing another pre-stored buffer solution through the column and directed to the detection assay. Fig. 4 shows a cartoon rendering of the pillar array with captured hmDNA molecules ( $\mathrm{CpG}$ regions are indicated with red balls) in the MBDfunctionalized (dark blue) silicon pillar array located in the microfluidic chamber.

\subsection{2 hm-DNA detection}

The purified and pre-concentrated hm-DNA samples are transported directly to the detection system, which consists of an array of silicon nanowire ( $\mathrm{Si}-\mathrm{NW}$ ) biosensors with probe molecules, specific for the epigenetic marker for colon cancer, attached to their surfaces. The probe molecules will be designed for a specific genetic sequence with methylation abnormality, as has been reported for 
colorectal cancer. ${ }^{16}$ Si-NW biosensors have been demonstrated to be highly sensitive to surface charge variations due to DNA/DNA ${ }^{17}$ hybridization and antibody/antigen binding. ${ }^{18}$

Current state of the art detection of ligand-target complex is based on fluorescence detection of targets labeled with fluorophore. The ligands are attached to a solid substrate and the target is contained in the sample solution. Fluorescence detection is very sensitive and requires an optical detection system, which is difficult to miniaturize. Fluorescent labels suffer from differences in labeling efficiencies, and photo bleaching effects. Chemical attachment of the fluorophore to the target, requires chemical processing steps, and can lead to alterations in ligand-target interactions caused by conformational changes or steric hindrance induced by the label and the labeling efficiency varies from protein to protein, making comparisons challenging. In addition, attaching fluorophores may influence the way in which proteins bind to other molecules and may cause increased background signals. ${ }^{19}$

For these reasons, there is considerable effort to investigate alternatives to fluorescence detection, called label-free detection. Label-free sensors detect the hybridization of an affinity complex using a variety of optical, mechanical, and electrochemical transduction methods. For the Nanopill, Si-NW biosensors have been developed and have been reported to be capable of providing highly sensitive label-free, real-time, electronic biomolecular detection, which could be directly interfaced to the electronic components of the Nanopill system. The unique sensing properties of these nanoscale sensors have recently been demonstrated by label-free detection of DNA hybridization of ssDNA and ssPNA receptor molecules anchored to the surface of oxidized Si-NWs. ${ }^{20-23}$ Recently, Hahm et al. ${ }^{20}$ reported the label-free electrical detection of peptide nucleic acid (PNA)-DNA hybridization. Si-NWs provide a compelling application for ultrasensitive label-free sensing of low quantity early stage cancer markers which is particularly important in the case of cancer therapies because early stages of the disease are typically treated with the greatest probability of success. ${ }^{24}$ The use of Si-NW sensors provides an ultrasensitive detection method using an entirely electrical measurement, which is suitable for miniaturization inside the Nanopill early warning cancer detection system. However, due to small amounts of hm-DNA contained in the sample, a preconcentration step or sample amplification step, using an integrated polymerase 
chain reaction system, would be required at the expense of increased complexity inside the Nanopill. Figure 5 shows a cartoon illustration of the DNA hybridization on the Si-NW biosensors. Si-NWs have been fabricated and electrically tested in the BIOS Group. On the surface of the Si-NWs the probe molecules would be immobilized using conventional silane linking chemistry. The probe molecules specific for a particular gene with methylation abnormality will be tailored for this application. The electronic detection of DNA using the ultra-small Si-NW biosensors inside the Nanopill, is the core of the Nanopill system. Figure 5 shows a cartoon of biomolecules binding to ligand molecules chemically anchored to the Si-NW surface.

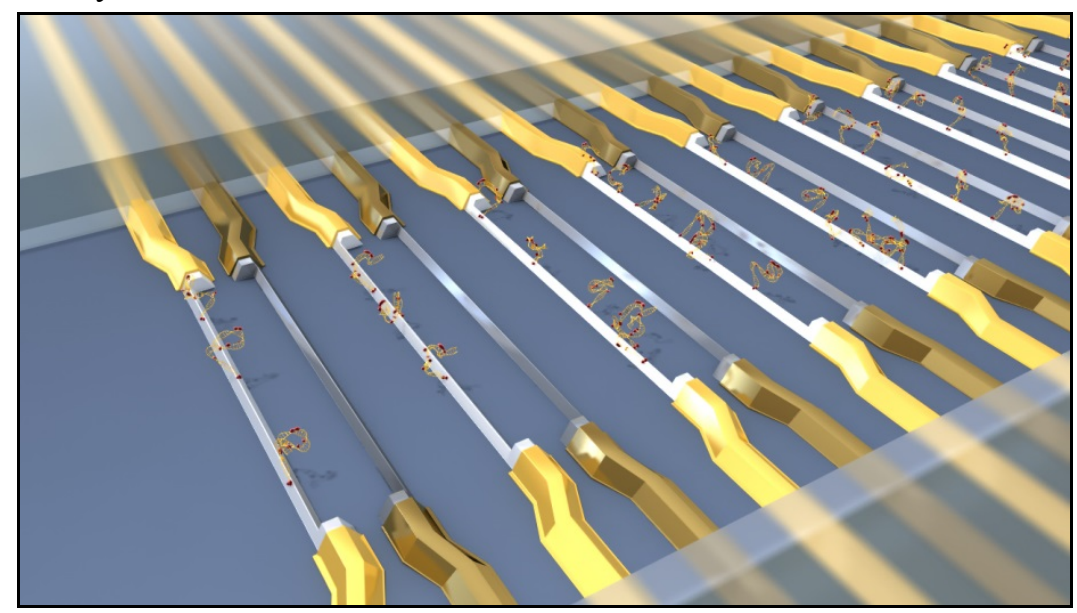

Figure 5. Cartoon illustration of specific DNA hybridization to probes with complementary sequence on the Si-NW biosensor array.

The molecular binding events changes the surface potential on the Si-NW surface, which due to field-effect is translated, into a detectable change of the electrical current flowing between the gold colored electrodes. Due to their small size, typically with cross-sectional area: $50 \times 50 \mathrm{~nm}^{2}$, small variations in the surface potential, or charge, can lead to large changes in the Si-NW electrical conductance. Most physiological sample serum contains ions, such as $\mathrm{NaCl}$ and $\mathrm{KCl}$ in concentrations ranging between $100 \mathrm{mM}$ to $200 \mathrm{mM}$, and smaller amounts of $\mathrm{MgCl}_{2}$ and $\mathrm{CaCl}_{2}$. Therefore, for optimal sensing performance (DNA hybridization efficiency and specificity) careful control of the surface potential is required using a reference electrode.

A significant amount of innovation has been incorporated into making 
good Si-NWs as part of a previous $\mathrm{PhD}$ thesis, ${ }^{25}$ where good working devices have been obtained, with a fabrication of high-density Si-NW arrays, avoiding complicated assembly techniques as needed for the bottom-up approach. The SiNWs have been realized using conventional top down microfabrication techniques with low electrical contact resistances. As part of this thesis, the main focus is the bioassay development using the Si-NW biosensors for DNA hybridization detection in an automated and integrated microfluidic platform. The key aspects of the bioassay development includes the development of the surface receptor linking chemistry, which includes covalent surface functionalization with probe molecules for hybridizing incoming DNA. Uncharged DNA mimic, synthetic peptide nucleic acid (PNA) molecules has been used as probes for DNA biomarker detection optimization. An important aspect of the technological development is the design and application of appropriate surface attachment chemistry of the ligands to the Si-NW gate-oxide surface. Depending on the type of ligand used for the experiment, i.e. oligonucleotides or PNAs, the appropriate ligand molecule can be purchased directly from a commercial source, such as Pepscan, b.v. or Eurogentec. The most commonly used anchoring technique uses a silane linking chemistry to the silicon dioxide gate surface, which provides an amine $\left(\mathrm{NH}_{2}\right)$ or carboxyl functional $(\mathrm{COOH})$ group for functionalization to any type of protein or DNA.

The integration of the Si-NW biosensors into a microfluidic platform is required to perform simultaneous fluid delivery and electronic measurements for the detection of PNA-DNA binding in a real-time flowing sample. The integrated platform ensures efficient PNA-DNA binding detection on Si-NWs, which could be eventually used in the low power consuming Si-NW sensor cartridge for the Nanopill. The integrated microfluidic platform could be suitably used for a wide range of small volume chemical and biochemical analyses. The integrated platform uses a disposable analysis cartridge consisting of the all-electrical Si-NW sensor array chip combined with a small volume microfluidic flow-cell such that only the analysis cartridge needs to be plugged in for each Si-NW biochemical sensing measurement. Sample delivery in the integrated analytical instrument is performed with an automated multi-sample injection system providing precise sample volumes together with elimination of erroneous sensor responses from sample switching due to flow rate fluctuations. 


\subsubsection{Electronics and wireless transmission}

The front-end electronic circuitry performs the acquisition of the Si-NW signals by means of conventional measurement techniques. A low-power microcontroller, the "brains" of the system, will keep track of various functions in the Nanopill such as timing, functional checks of the subsystems and the actual data processing. The output electronics would consist of a commercially available wireless transmission chip, which will transmit in the radiowave frequency spectrum. Low power consumption radio transmitters, which are currently equipped with microcontrollers for digital processing of radiowave transmission protocols is needed for the Nanopill notification system. The Zigbee protocol is developed for low power applications, and its microcontroller is designed such that it also has low power consumption. The MSP430 chip (Texas Instruments) that is on the Zigbee platform is very low power consuming and extremely small sized, and would be more than capable in performing the necessary tasks, described for the Nanopill system. Most Zigbee platforms, however, have the downside of having their carrier frequency at $2.4 \mathrm{GHz}$, which is near the absorption frequency of water molecules. This will negatively influence the transmission efficiency and as such will increase the power consumption for a given distance between the pill and the receiver. However the Zigbee platform also provides a carrier frequency in the $868 \mathrm{MHz}$ band. Other alternatives are available, such as a modification kit that uses a carrier frequency in the 868/915 MHz band (Amber 8423, Amber Technologies). Recently, Valdestry $e t$ al. $^{26}$ demonstrated that radio link can be transmitted between the intestinal tract of the pig with a receiver located outside using the standard Zigbee kit and their results were promising. The development and implementation of the electronics and wireless transmission have been performed by Dr. Wouter Sparreboom and Dr. Ad Sprenkels from the BIOS Group.

\subsubsection{Electrical power supply}

The electrical power for sample extraction, transportation, detection, electronic processing and wireless transmission will be supplied by a commercial high energy density battery (CR927 lithium button cell with a capacity of 30mAh, Duracell: DL927 or DL927B), which can easily provide enough energy for all functions and operations. 


\subsubsection{Integrated system}

For the integrated system, a pump and valves would be necessary components to perform the automated microfluidic functions of the Nanopill. As previously described, the intestinal fluid is collected in the intestinal tract, which is the external environment of the Nanopill. The collection of the intestinal fluid is to be performed with an integrated micropump that is capable of withdrawing an adequate amount of intestinal fluid and mixing with a pre-stored dilution buffer solution. The microfluidic system contains a silicon micromachined extraction column with high surface area for capturing the hm-DNA directly from the diluted sample. The captured DNA would be released by flowing a pre-stored elution buffer solution through the column and directly to the detection assay.

An electromagnetically actuated peristaltic pump has been developed for the Nanopill. Although electromagnetic actuators have been previously applied to peristaltic pumps,${ }^{27}$ they are typically large in size and require high activation currents, therefore, a low power voice-coil motor (VCM) electromagnetic actuator is used, which were originally developed by the hearing aid industry. They have been optimized in size and power consumption, which are both very important parameters in the application in the pill. An electromagnetically actuated (FK series VCM, Knowles) peristaltic pump (3 actuators per pump) was implemented with a total volume of approximately $75 \mu \mathrm{l}(25 \times$ less than the total volume of the pill). The power consumption of the three actuators producing a of flow rate of approximately $1 \mu \mathrm{l} / \mathrm{min}$ is $4.2 \mathrm{~mW}$ at $1 \mathrm{~V}$, which gives approximately 23 hours of operation on a $3 \mathrm{~V}, 30 \mathrm{mAh}$ battery. The development and implementation of the peristaltic pump have been performed by Dr. Wouter Sparreboom and Dr. Ad Sprenkels of the BIOS Group. 


\subsection{Conclusion}

The system components to perform an automated miniaturized sample preparation for a hyper-methylated bioassay for colorectal DNA marker electronic detection with a pump, valves, mixing chambers, with microcontroller monitoring such that a final readout is transmitted as a wireless signal are feasible to be integrated together to realize the Nanopill diagnostic system. However, the development of the entire Nanopill system is beyond the scope of a single doctoral thesis. This thesis focuses on the development and realization of an automated microscale bioassay for the capture and enrichment of the hmDNA colorectal cancer marker and the electronic detection of DNA using SiNW biosensors, as part of a two-step assay for cancer marker detection that can be eventually integrated into the Nanopill system. 


\section{References}

1. A van den Berg, A Sprenkels, E.T. Carlen, Blue Bolus Chip proposal June 2007.

2. R. Siegel, D. Naishadham and A. Jemal, CA Cancer Journal for Clinicians, 2012, 62, 10-29.

3. D. M. Parkin, L. Boyd and L. C. Walker, British Journal of Cancer, 2011, 105, S77-S81.

4. L. Migliore, F. Migheli, R. Spisni and F. Copped, Journal of Biomedicine and Biotechnology, 2011, 2011.

5. M. Paulsen and A. C. Ferguson-Smith, Journal of Pathology, 2001, 195, 111-134.

6. L. J. W. Bosch, B. Carvalho, R. J. A. Fijneman, C. R. Jimenez, H. M. Pinedo, M. Van Engeland and G. A. Meijer, Clinical Colorectal Cancer, 2011, 10, 8-23.

7. G. Lyratzopoulos, D. C. Greenberg, G. P. Rubin, G. A. Abel, F. M. Walter and R. D. Neal, Expert Review of Anticancer Therapy, 2012, 12, 993-996.

8. P. Bird, Nature, 1986, 321, 209-213.

9. J. G. Herman, F. Latif, Y. Weng, M. I. Lerman, B. Zbar, S. Liu, D. Samid, D. S. R. Duan, J. R. Gnarra, W. M. Linehan and S. B. Baylin, Proceedings of the National Academy of Sciences of the United States of America, 1994, 91, 9700-9704.

10. J. G. Herman, J. R. Graff, S. Myöhänen, B. D. Nelkin and S. B. Baylin, Proceedings of the National Academy of Sciences of the United States of America, 1996, 93, 9821-9826.

11. A. Eads, K. D. Danenberg, K. Kawakami, L. B. Saltz, C. Blake, D. Shibata, P. V. Danenberg and P. W. Laird, Nucleic Acids Research, 2000, 28.

12. J. P. J. Issa, Y. L. Ottaviano, P. Celano, S. R. Hamilton, N. E. Davidson and S. B. Baylin, Nature Genetics, 1994, 7, 536-540.

13. Nestor, A. Ruzov, R. R. Meehan and D. S. Dunican, BioTechniques, 2010, 48, 317-319.

14. Gebhard, L. Schwarzfischer, T. H. Pham, E. Schilling, M. Klug, R. Andreesen and M. Rehli, Cancer Research, 2006, 66, 6118-6128.

15. T. Rauch, H. Li, X. Wu and G. P. Pfeifer, Cancer Research, 2006, 66, 79397947. 
16. M. Weber, J. J. Davies, D. Wittig, E. J. Oakeley, M. Haase, W. L. Lam and D. Schübeler, Nature Genetics, 2005, 37, 853-862.

17. Y. L. Bunimovich, Y. S. Shin, W. S. Yeo, M. Amori, G. Kwong and J. R. Heath, Journal of the American Chemical Society, 2006, 128, 16323-16331.

18. G. Zheng, F. Patolsky, Y. Cui, W. U. Wang and C. M. Lieber, Nature Biotechnology, 2005, 23, 1294-1301.

19. Hitt, Drug discovery and development, 2007.

20. J. I. Hahm and C. M. Lieber, Nano Letters, 2004, 4, 51-54.

21. J. Zhang, G. Zhang, J. H. Chua, R. E. Chee, E. H. Wong, A. Agarwal, K. D. Buddharaju, N. Singh, Z. Gao and N. Balasubramanian, Nano Letters, 2008, 8, 1066-1070.

22. Z. Gao, A. Agarwal, A. D. Trigg, N. Singh, C. Fang, C. H. Tung, Y. Fan, K. D. Buddharaju and J. Kong, Analytical Chemistry, 2007, 79, 3291-3297.

23. T. Uno, H. Tabata and T. Kawal, Analytical Chemistry, 2007, 79, 52-59.

24. M. M.-C. Cheng, G. Cuda, Y. L. Bunimovich, M. Gaspari, J. R. Heath, H. D. Hill, C. A. Mirkin, A. J. Nijdam, R. Terracciano, T. Thundat and M. Ferrari, Current Opinion in Chemical Biology, 2006, 10, 11-19.

25. S. Chen, Silicon Nanowire Field-Effect Chemical Sensor, Doctoral Thesis, University of Twente, The Netherlands, 2012.

26. P. Valdastri, A. Menciassi and P. Dario, IEEE Transactions on Biomedical Engineering, 2008, 55, 1705-1710.

27. M. J. Mescher, C. Dube, M. Varghese and J. Fiering, Surface mount microfluidic flow regulator on a polymer substrate, 2003. 
Chapter 3 


\section{Chapter 4}

\section{Target enrichment using microfluidic solid phase extraction $^{1}$}

Clinical point of care testing (POCT) systems for early cancer screening markers are currently in their development infancy. Genetic sequence and hypermethylation profile information from the promoter regions of tumor suppressor genes are both recognized as important for molecular cancer disease investigation. Since hyper-methylated DNA (hm-DNA) is typically present in ultra-low concentrations in biological samples, such as stool, urine, and saliva, sample enrichment and amplification is required before detection with conventional analysis tools is possible. Currently, hm-DNA enrichment kits containing methyl binding domain (MBD) proteins that bind specifically to hmDNA regions are commercially available, and several new hm-DNA assays have been developed. In this chapter, we present a small volume microfluidic chip for low concentration ( $\leq 1 \mathrm{ng} \mathrm{mL}^{-1}$ ) capture and elution of hm-DNA using a passive silicon-based protein-DNA capture and elution platform. All assay steps have been qualitatively characterized using a real-time surface plasmon resonance (SPR) biosensor. A capture/elution efficiency of $84 \%$ from the assay has been quantitatively characterized using calibrated fluorescence spectroscopy.

\footnotetext{
${ }^{1}$ Adapted from: A. De, W. Sparreboom, L. de Vreede, E.T. Carlen and A. van den Berg, Small Volume Hyper-methylated DNA Enrichment for Epigenetics, The 16th Intl. Conf. on Miniaturized Systems for Chemistry and Life Science (MicroTAS 2012), October 28November 1, 2012, Okinawa, Japan.
} 


\subsection{Introduction}

Microfluidic, lab-on-a-chip systems are emerging for clinical and molecular biology applications ${ }^{1}$ where all assay steps can ideally be combined into a single, miniaturized device. A main advantage of a lab-on-a-chip platform is the reduction of sample volume of expensive reagents that involve multiple sequential reaction, washes and long incubation times. Traditional surface attached ligand-ligate techniques, such as the enzyme linked immunosorbent assay (ELISA) ${ }^{2}$, southern blot, ${ }^{3}$ western blot, ${ }^{4,5}$ microarray hybridization detection, ${ }^{6-8}$ for detection of DNA, proteins, RNA and small molecules, respectively have been realized into miniaturized microfluidic versions. There have been several reports of other applications such as whole microbial genetic material analysis on microfluidic chip, ${ }^{9}$ PCR integration, ${ }^{10-12}$ solid phase separation of nucleic acids, cells, and proteins from complex mixtures ${ }^{13-19}$ as well as solid phase amplification and on-chip sequencing. ${ }^{20,21}$

The field of microfluidics involves the manipulation of fluids within microchannel networks manufactured in a solid substrate, such as glass, silicon or plastic, with characteristic channel dimensions varying between $10-100 \mu \mathrm{m}$ and sample volumes in 10-100 $\mathrm{nL}$ volume range. The pressure driven flow of an aqueous solution inside a microchannel is laminar $(R e<<1)^{2}$ with a parabolic flow velocity profile. Due to the laminar flow regime, mixing when not controlled occurs only through diffusion. The small channel dimensions results in minimal reagent consumption and waste production, minimizing costs of reagent. Reduced device footprints, with low power consumption and manufacturing costs (depending on material) due to potential for mass production are added benefits of using microfluidic technology.

A common strategy for reducing assay times, with increased capture efficiency, consists of utilizing analyte diffusion across large surface areas within microchannels with the use of pillars, posts or microparticle structures. The most common technique for analyte capture is the use of superparamagnetic microparticles that are manipulated with external magnets.

\footnotetext{
${ }^{2}$ The Reynolds number $R e=\rho U_{a} D_{h} / \mu$, where $\rho$ is the fluid density, $\mu$ is the dynamic viscosity of the fluid, $U_{\mathrm{a}}$ is the mean flow speed, $D_{h}$ is the hydraulic diameter of the flow channel, which for a rectangular microchannel is $4 A / P$, where $A$ is the area of cross-section and $P$ is the perimeter, e.g. a microchannel of square cross-section with $50 \mu \mathrm{m}$ height, $D_{h}=5 \times 10^{-7} \mathrm{~m}$. For a water sample, $\mu=9 \times 10^{-4} \mathrm{~Pa}-\mathrm{s}$ and $\rho=1000 \mathrm{~kg} \mathrm{~m}^{-3}$, and assuming $U_{a}=0.5 \mathrm{~mm} \mathrm{~s}^{-1}$, then $R e=0.03$.
} 
Super-paramagnetic microparticles are only magnetic in the presence of a magnetic field and removal of the guiding field allows their diffusion into the sample solution. ${ }^{22}, 23$ Several applications that use super-paramagnetic microparticles as solid supports have been reported for immunoassays, ${ }^{24-26}$ DNA $^{27,} 28$ and RNA hybridization, ${ }^{29,} 30$ tryptic digestion, ${ }^{31}$ and cell manipulation. ${ }^{32,33}$

Although magnetic particle based assays have the advantage of operation simplicity, their implementation, typically requires large volume reagent and washing solutions, which characterize them as miniaturized batch processes, and additionally, their implementation in a microfluidic format is not straight forward. Therefore, the particle based methods suffer from the same inherent inconsistencies as found in large lab-scale assays. ${ }^{34}$ Hence, passive microfluidic structures with pillars or other microfabricated features with increased capture area, within reduced dimensions, are commonly used for integrated applications for multi-step analytical applications. Microfabricated integrated systems for PCR with capillary electrophoresis, ${ }^{35-37}$ combined genetic assay, ${ }^{38}$ blood sample preparation with PCR, ${ }^{39}$ integrated Sanger sequencing ${ }^{40}$ have been reported. For epigenetics, small volume solid-phase hm-DNA capture and elution systems are becoming increasingly important as opposed to conventional large volume magnetic bead based solution assay products.

In this chapter, we realize a small volume solid-phase hm-DNA extraction (capture and elution) chip in a microfabricated lab-on-chip platform that is intended to be used in the Nanopill diagnostic system, which requires a simple and low power sample processing module. Fig. 1 gives a schematic overview of the conceptual hm-DNA extraction system for Nanopill diagnostic system, which includes a microfluidic pump, internal buffer reservoirs (WBR and EBR), a sample mixer, microfluidic valves (SV and EV), DNA detection system, and the capture/elution microfluidic chip. The microfluidic capture and elution chip design is based on a conventional structure that interrupts the laminar flow boundary conditions using rotated pillar arrays inside the microchannel. The conceptual operation of the Nanopill sample extraction system involves sampling of the intestinal fluid with elution valve (EV) closed, sample valve (SV) open, and active pumping on, to the waste outlet. The intestinal fluid would be mixed with a wash buffer, from the wash buffer reservoir (WBR), followed by the transport to the capture/elution chip where the 
hm-DNA is captured by the MBD protein pillar array and the remaining sample fluid is transported out of Nanopill through the waste outlet.

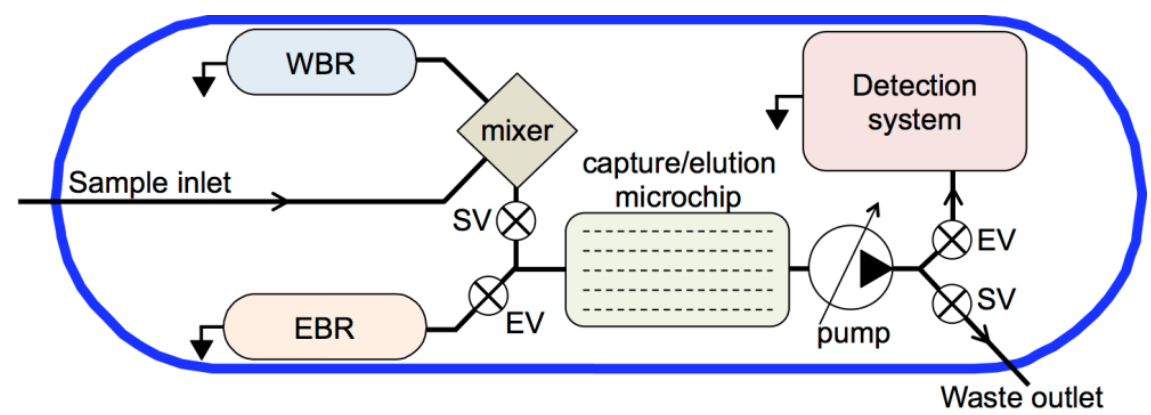

Figure 1. Schematic diagram of the concept microfluidic sample capture/elution architecture for the Nanopill diagnostic system.

The chip contains large surface area microchannels functionalized with a commercially available methyl binding domain (MBD) protein. The MBD protein coated surfaces are used to specifically capture hm-DNA, which is then subsequently eluted. Since the amount of hm-DNA present in the intestinal fluid is expected to be very small, a large volume of intestinal fluid will be required. After completion of the capture for an adequate time duration, the sample valves $(\mathrm{SV})$ is closed and elution valves (EV) opened to elute the specifically captured hm-DNA from the MBD functionalized surfaces with the elution buffer from the elution buffer reservoir (EBR) into the detection chamber.

The focus of this chapter is to develop and characterize the hm-DNA capture and elution protocol using a small volume microfluidic platform, function. The microfluidic chip fabrication, surface functionalization, assay implementation is demonstrated for low concentration hm-DNA samples. The microfluidic hm-DNA capture/elution chip can be further miniaturized and optimized for integration with the silicon nanowire detection system. Table 1 compares the solid phase microscale capture/elution efficiency of MBD chip with respect to three commercially available magnetic particle based kits, i.e. Methyl Collector Ultra, (Active motif), Epimark (New England Biolabs) and Methyl Miner, (Invitrogen). The commercial kits for hm-DNA enrichment recommend the use of $\mu \mathrm{g}$ amounts of DNA with large volume washes (1-3 $\mathrm{mL})$, and end-point detection with bisulfite sequencing followed by either methylation independent or methylation specific PCR for CpG status analysis. 
Table 1. Comparison of commercial DNA extraction kits with MBD DNA extraction microchip.

\begin{tabular}{|c|c|c|c|c|}
\hline Characteristic & Epimark & $\begin{array}{l}\text { Methyl } \\
\text { Collector }\end{array}$ & Methyl Miner & $\begin{array}{c}\text { MBD } \\
\text { microchip }\end{array}$ \\
\hline $\begin{array}{c}\text { DNA amount } \\
\text { (ng) }\end{array}$ & $5-10,000$ & $1-1000$ & $5-10,000$ & $\leq 1$ \\
\hline $\begin{array}{l}\text { Handling } \\
\text { volume }\end{array}$ & $100 \mu \mathrm{L}$ & $100 \mu \mathrm{L}$ & $100 \mu \mathrm{L}$ & $1 \mathrm{~mL}$ \\
\hline ds-DNA & Yes & Yes & Yes & Yes \\
\hline $\begin{array}{c}\text { Concentration } \\
\left(\text { ng } \mathrm{mL}^{-1}\right)\end{array}$ & $50-10^{5}$ & $1-10^{4}$ & $1-10^{4}$ & $>1$ \\
\hline $\begin{array}{c}\text { Protein } \\
\text { configuration }\end{array}$ & MBD2a-Fc & $\begin{array}{c}\text { His- } \\
\text { MBD2b/MBD } \\
\text { 3L1 }\end{array}$ & MBD2b-biotin & $\begin{array}{l}\text { MBD2b- } \\
\text { biotin }\end{array}$ \\
\hline $\begin{array}{l}\text { Wash/incubati } \\
\text { on buffer }\end{array}$ & $\begin{array}{c}150 \mathrm{mM} \\
\mathrm{NaCl}\end{array}$ & $\begin{array}{l}\text { Low and high } \\
\text { salt capture }\end{array}$ & $160 \mathrm{mM} \mathrm{NaCl}$ & $\begin{array}{c}160 \mathrm{mM} \\
\mathrm{NaCl}\end{array}$ \\
\hline $\begin{array}{c}\text { Wash volume } \\
(\mathrm{mL})\end{array}$ & 3 & 1 & 0.4 & 0.4 \\
\hline $\begin{array}{c}\text { Elution volume } \\
(\mu \mathrm{L})\end{array}$ & 50 & $\begin{array}{c}100 \text { (with } \\
\text { proteinase K) }\end{array}$ & 400 & 25 \\
\hline $\begin{array}{c}\text { Fragment } \\
\text { length }\end{array}$ & $<1000 \mathrm{bp}$ & $<1000$ bp & $<1000 \mathrm{bp}$ & $80 \mathrm{bp}$ \\
\hline $\begin{array}{l}\text { Capture } \\
\text { method }\end{array}$ & $\begin{array}{c}\text { Magnetic } \\
\text { beads- } \\
\text { protein-A }\end{array}$ & $\begin{array}{l}\text { Magnetic-Ni } \\
\text { beads }\end{array}$ & $\begin{array}{l}\text { Dynabeads- } \\
\text { M280-SA }\end{array}$ & $\begin{array}{l}\text { MBD-Si- } \\
\text { pillar array }\end{array}$ \\
\hline $\begin{array}{l}\text { Elution } \\
\text { protocol }\end{array}$ & $\begin{array}{c}\text { DNase free } \\
\text { water } 65^{\circ}\end{array}$ & $\begin{array}{c}\text { Gradient } \mathrm{NaCl} \\
200 \mathrm{mM}-2 \mathrm{M} \\
\end{array}$ & $\begin{array}{c}\text { Gradient } \mathrm{NaCl} \\
200 \mathrm{mM}-2 \mathrm{M} \\
\end{array}$ & $2 \mathrm{M} \mathrm{NaCl}$ \\
\hline $\begin{array}{c}\text { Elution } \\
\text { efficiency }\end{array}$ & - & - & $70-90 \%$ & $84-92 \%$ \\
\hline $\begin{array}{c}\text { Concentration } \\
\text { factor }\end{array}$ & $\begin{array}{l}500-1000 \times \\
(\mathrm{qPCR})\end{array}$ & $\begin{array}{c}10 \times \\
(q P C R)\end{array}$ & - & $\begin{array}{c}28 \times \\
\text { (fluore } \\
\text { scence) }\end{array}$ \\
\hline
\end{tabular}

The microscale MBD chip performs with a capture-elution efficiency greater than $90 \%$ matching with the reported large volume efficiency of Methyl Miner kit. For the magnetic bead assays elution efficiencies can be increased to greater than $95 \%$ by repeated elution from the magnetic bead surfaces. The kits use small handling volume for incubating DNA with MBD protein compared to the MBD chip application as the focused is on enriching hm-DNA from concentrated samples. The MBD chip deals with large volume dilute low concentration hm-DNA. The MBD chip can process sample hm-DNA (concentration less than $1 \mathrm{ng} \mathrm{mL}^{-1}$ ) and concentrate 28-fold eluting into a small 
volume of $25 \mu \mathrm{L}$. All experiments have been performed with 80 -bp ds-hm-DNA with eight symmetric $\mathrm{CpG}$ islands, which is a good size to characterize the MBD system as fragments larger than 75 bp can be easily amplified with PCR. ${ }^{41}$ Typically, enzymatically digested genomic DNA or DNA fragmented by sonication in the range of $<1000 \mathrm{bp}$ is recommended for the solution based magnetic bead assays. This kind of small volume, microscale solid phase epigenetic enrichment platform with high capture elution efficiency and concentration factor is necessary for integrated on-chip hm-DNA analysis.

\subsection{Experimental}

\subsubsection{Surface functionalization}

The MBD protein functionalization scheme on silicon dioxide $\left(\mathrm{SiO}_{2}\right)$ surfaces is shown in Scheme. 1.

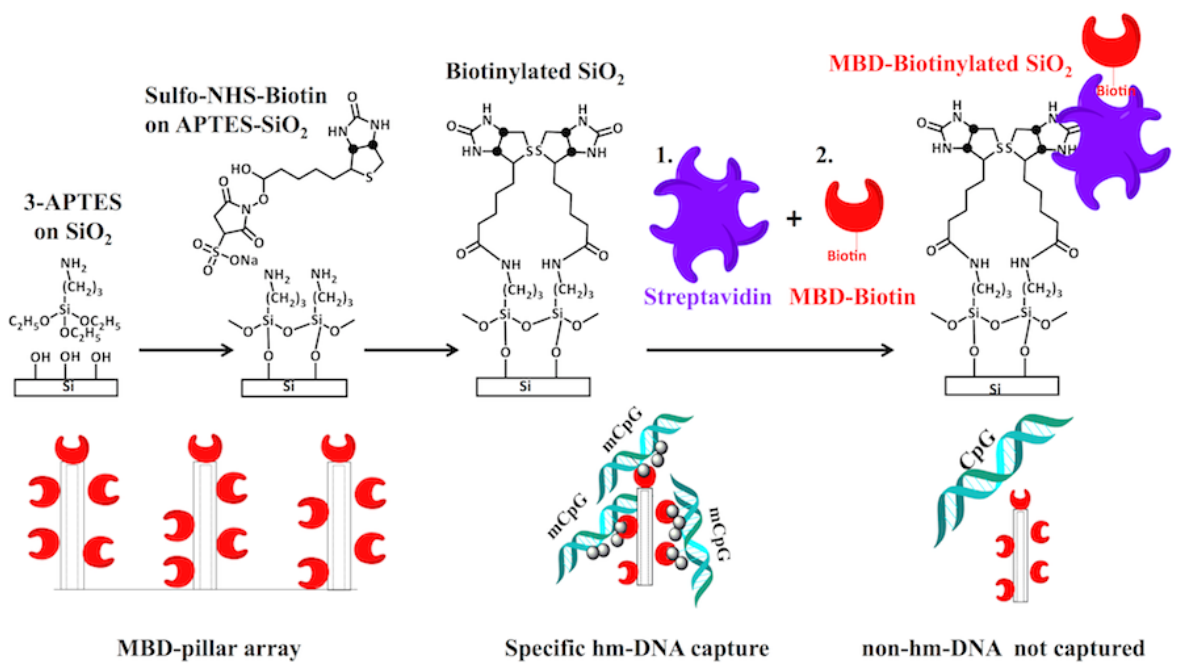

Scheme 1. Surface functionalization scheme for hm-DNA capture using MBD proteins on $\mathrm{SiO}_{2}$ surfaces.

An amine functional group is first covalently attached to the $\mathrm{SiO}_{2}$ surface using 3 -amino propyl trimethoxy alkyl silane (APTES). Following immersion in a 2\% APTES solution, the glass-silicon chip was washed with ethanol and heated at $120{ }^{\circ} \mathrm{C}$ for $15 \mathrm{~min}$ prior to further coupling steps. A biotinylated surface is formed using a $10 \mathrm{mM}$ biotin-N-hydroxysuccinimide ester in phosphate buffered saline (PBS) buffer. The biotinylated surface was exposed to a $1 \mu \mathrm{M}$ streptavidin 
(SA) solution. The biotinylated MBD protein is then attached to the available sites on the SA covered surface. The MBD protein is specifically the MBD2b protein, with mass of $29 \mathrm{kDa}$, was used from a commercially available kit (MethylMiner $^{\mathrm{TM}}$ Methylated DNA Enrichment Kit, Invitrogen/Life Technologies). All functionalization steps were done for $30 \mathrm{~min}$ by flowing the liquid inside the channel and stopping the flow for $30 \mathrm{~min}$, and followed by surface washes with the respective solvents. The DNA used for capture is $80 \mathrm{bp}$ ds-DNA with eight symmetric 5-methyl-CpG islands and molecular weight $49537 \mathrm{~g} \mathrm{~mol}^{-1}(\approx 50 \mathrm{kDa})$. Fig. 2 shows the sequence of the $80 \mathrm{bp}$ ds-DNA with the eight symmetrically methylated $\mathrm{CpG}$ islands. The surface functionalization of the $\mathrm{SiO}_{2}$ surface with MBD2b was observed with a fluorescent label (SA488). The MBD2b protein is supplied at $0.5 \mathrm{mg} \mathrm{mL}^{-1}$ concentration and diluted to $35 \mu \mathrm{g} \mathrm{mL}^{-1}$ for further use. An 80-bp non-hm-DNA, with the same sequence as the sample $80 \mathrm{bp}$ ds-DNA, was used as the control DNA. The concentrations of the DNA and MBD2b proteins were measured before start of experiments using a spectrophotometer (Nanodrop 2000c).

5-GCT ATA CAG GGMGTG TTA AMG ATA TAA MGT TTT GGC TMG ACC AGT GAC MGG ACT CT M G TT CCT ACC AGM G CA A MG CCCCC-3 3-CGA TAT GTC CCGM AC AAT TGM TAT ATT GMA AAA CCG AGM TGG TCA CTG GMC TGA GA G M AA GGA TGG TCGMGT TGMGGGGG-5

Figure 2. 80 bp ds-DNA with eight symmetric 5-mCpG islands, denoted by ' $M$ ' and used for hm-DNA capture experiments. ${ }^{42}$

Following the preparation of the silicon-glass chip with the MBD2b capture proteins, the chip was used to capture hm-DNA. Briefly, the MBD2b chip was loaded with hm-DNA in the incubation/wash buffer consisting of 160 $\mathrm{mM} \mathrm{NaCl}$ for $1 \mathrm{hr}$ by flowing the $1 \mathrm{~mL}$ load volume through the chip. A wash step with $100 \mu \mathrm{L}$ buffer is followed by an elution step with $1 \mathrm{~mL}$ high ionic strength elution buffer consisting of $2 \mathrm{M} \mathrm{NaCl}$ flowed at 1 bar. The incubation buffers, MBD2b proteins, and elution buffers were used from the Methyl Miner Kit. ${ }^{43}$ The microfluidic chips have been used for the capture and elution of hmDNA at various concentrations. The eluted DNA was de-salted with ethanol precipitation and re-suspended in $1 \mathrm{~mL}$ wash buffer and the concentration measured with pico-green intercalating dye fluorescence assay (Quant-IT, Invitrogen).

\subsubsection{Fluorescence assay quantification}

A spectrometer (Perkin Elmer) was used to measure the fluorescence from re- 
suspended eluted DNA in a final $2 \mathrm{~mL}$ volume. The calibration curve shown in Fig. 3 is used for quantification of the capture/elution assay. Using the calibration curve for known concentrations of hm-DNA, the concentration as output from the MBD chip was quantified by dilution to a $2 \mathrm{~mL}$ volume for fluorescence measurements.

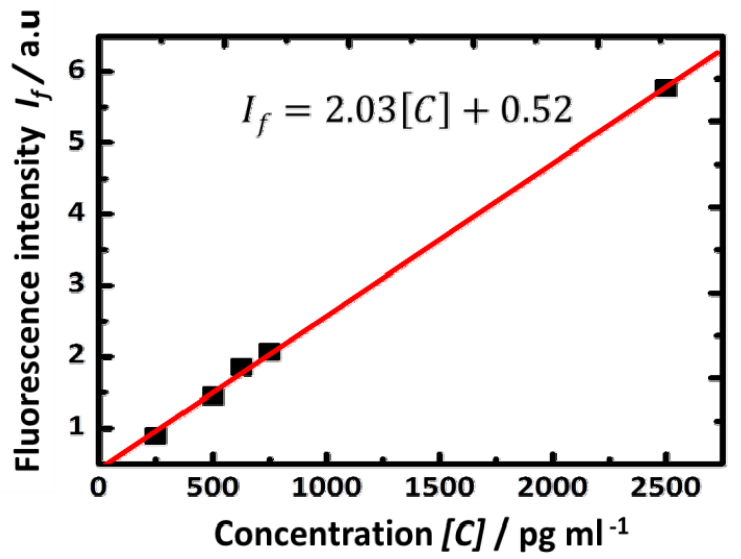

Figure 3. ds-hm-DNA pico-green assay calibration curve.

\subsubsection{Real-time assay monitoring}

A commercially available SPR biosensor instrument (SPRimager®II Array Instrument, GWC Technologies) is used to demonstrate the MBD2b selectivity for specific hm-DNA over non-hyper-methylated (non-hm-DNA), as well as the validation of the general capture and elution assay protocol. The SPR measurements were done using thiolated reagents conjugated to gold-coated substrates (SPRchip ${ }^{\mathrm{TM}}$ substrates, GWC Technologies).

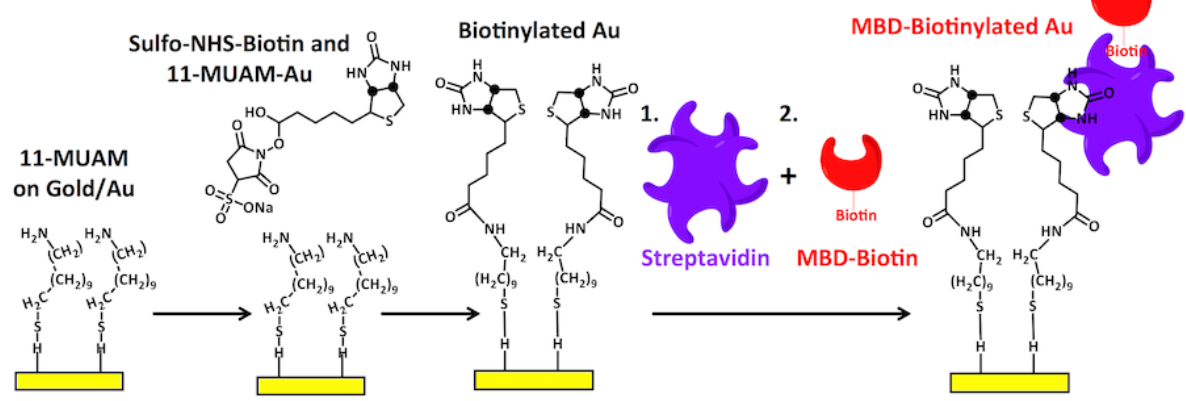

Scheme 2. Surface functionalization scheme for hm-DNA capture using MBD2b proteins on Au surfaces. 
The SPR chips were first cleaned in a fresh piranha solution $\left(\mathrm{H}_{2} \mathrm{SO}_{4}: \mathrm{H}_{2} \mathrm{O}_{2}, 3: 7\right)$ for $3 \mathrm{~min}$ prior to any surface functionalization, and subsequently incubated in a $5 \mathrm{mM}$ ethanolic 11-mercapto-undecylamine (MUAM, Dojindo) solution overnight ( $\sim 12 \mathrm{hrs}$ ) to form the MUAM monolayer. The MUAM treated gold SPR disks were next treated with biotin N-hydroxysuccinimide (NHS) for 30 min in $1 \times$ PBS buffer $\mathrm{pH} 7.4$, followed by incubation with $1 \mu \mathrm{M}$ streptavidin (SA), which is used to conjugate the MBD2b-biotin proteins to the SPR surface. Scheme 2 shows the complete SA conjugation to the Au SPR surfaces.

The MUAM-biotin-SA-biotin-MBD2b sandwich complex is a simple surface functionalization using the biotinylated MBD2b protein. The small equilibrium dissociation coefficient $\left(K_{\mathrm{D}} \sim \mathrm{fM}\right)$ of the biotin-SA conjugate provides a very robust complex. Since the MBD2b protein was available in a limited amount, the surface preparation and assay steps were performed in a microchannel to reduce reagent consumption.

Each step of the MUAM-biotin-SA-biotin-MBD2b complex formation was monitored using real-time SPR measurements. Subsequently, the MBD2b treated SPR disks were reacted with hm-DNA, non-hm-DNA, and elution protocol, respectively. The basic assay protocol is borrowed from the MethylMiner kit. ${ }^{43}$ All handling, incubation of hm-DNA and washing is done in $1 \times$ wash buffer, i.e. buffer containing $160 \mathrm{mM} \mathrm{NaCl}$ ionic strength. A $5 \mathrm{~mL}$ concentrated $5 \times$ wash buffer is provided with the kit and diluted for use. Elution is done with the high ionic strength buffer containing $2 \mathrm{M} \mathrm{NaCl}$.

\subsubsection{Chip microfabrication}

The bonded glass-silicon microchip was microfabricated. A $50 \mu \mathrm{m}$ deep channel was etched in silicon using deep reactive ion etching from a fast etching recipe to form high aspect ratio pillar structures. This was followed by growth of a 300 $\mathrm{nm}$ thick $\mathrm{SiO}_{2}$ layer. The thickness of the $\mathrm{SiO}_{2}$ layer should be sufficient to avoid quenching of fluorescent biomolecules. A $1.1 \mathrm{~mm}$ thick borofloat glass wafer is covered with polymer foil (BF410) and exposed for $15 \mathrm{~s}$ with UV radiation through lithography mask and developed with bicarbonate solution to form the openings in the polymer foil where the through holes in the glass wafer were formed using powder blasting. The powder blasting is done in a custom made setup with $29 \mu$ m diameter $\mathrm{Al}_{2} \mathrm{O}_{3}$ particles. 
Silicon wafer

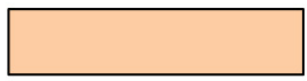

Photoresist

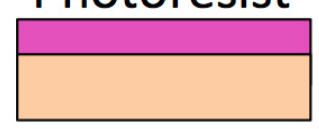

\section{Lithography}
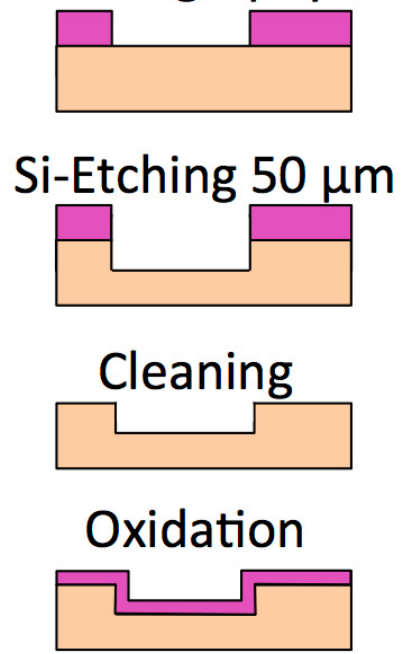

Borofloat glass

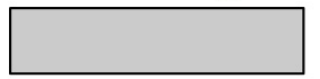

Lithography

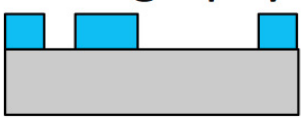

\section{Powder blasting}
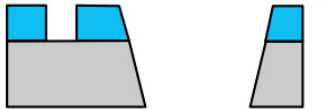

Anodic bonding

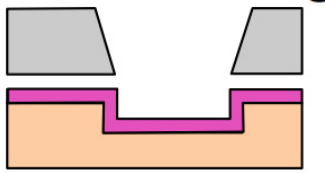

Bonded Si-Glass

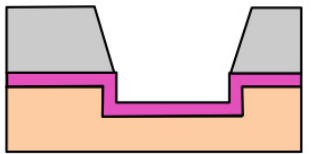

Figure 4. Fabrication flow chart for glass-silicon MBD chip.

The powder blasted glass wafer was cleaned by sonication in deionized water. The Si-glass chips were manufactured by anodically bonding the borofloat glass wafer with inlet-outlet holes to the silicon wafer containing the microfluidic channels. The bonded glass-silicon wafer is diced into $10 \times 20 \mathrm{~mm}^{2}$ pieces. Fig. 4 shows a schematic of the entire fabrication process for the glass-silicon MBD capture chip.

\subsubsection{Assay test system}

The diced chips are clamped in a custom-made chip holder. Fluidic connections are made to the chip with microfluidic scale fittings (Nanoport, Upchurch) and $150 \mu \mathrm{m}$ inside diameter (ID) poly-ether-ketone (PEEK) tubing (Upchurch). The fluid transported through the chip assembly with a regulated pressure source and controller (MFCS-8C, Fluigent). All surface functionalization is performed 
within the microchannels of the silicon-glass chip. The MBD2b capture of hmDNA is performed by flowing $1 \mathrm{~mL}$ of hm-DNA (with concentration $<1 \mathrm{ng} \mathrm{mL}-$ ${ }^{1}$ ) through the chip for $1 \mathrm{hr}$ (pressure $\Delta P=100 \mathrm{mbar}$ ), and followed by washing and elution. The incubation and washing are performed at the same ionic strength of $160 \mathrm{mM} \mathrm{NaCl}$ buffer. The elution step is performed with a buffer containing $2 \mathrm{M} \mathrm{NaCl}$ ionic strength at high flow rate $Q=200 \mu \mathrm{L} \mathrm{min}^{-1}$ ( $\Delta P=1$ bar) for $1 \mathrm{~mL}$ elution volumes. For small volume elution, the sample flow rate was $Q=2.5 \mu \mathrm{L} \mathrm{min} \operatorname{mon}^{-1}(\Delta P=100 \mathrm{mbar})$. Fig. 5 shows images of the MBD microchip assay test system.

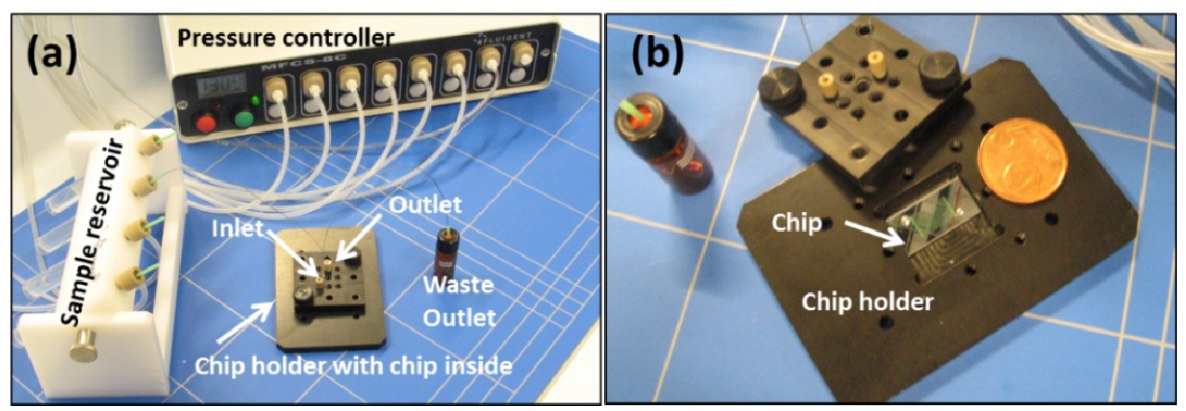

Figure 5. Experimental setup for the MBD capture/elution chip testing (a) complete test system (b) MBD microchip mounted in chip holder.

\subsection{Results and Discussion}

\subsubsection{Chip design}

Pressure driven flow in microfluidic channels is laminar with a parabolic flow velocity profile where diffusion predominates on the channel walls. ${ }^{44}$ Due to the parabolic flow profile, a majority of the analyte flow in the microchannel is not utilized as interaction occurs only at the channel walls, where the flow velocity is ideally zero and balanced diffusion-convection-reaction is required. A large microchannel surface area that is in contact with analyte sample flow is required in order to maximize the capture capacity of the microchip extraction system. There are several approaches for increasing channel surface area and to break the boundary condition of static flow on channel walls like using microparticle, sol-gel material, making pillar arrays within the micro-channel to enhance mixing and reagent transport.

Two different chip designs have been considered, and are shown in Figs. 6(a) and 6(b). These designs have been previously demonstrated for solid 
phase DNA extraction platforms. ${ }^{19,45}$ Many reports of progress have been made in the field of solid phase DNA extraction platforms with different structures, ${ }^{46}$, 47 and integrated designs. ${ }^{48,} 49$ However, we focus on a microfabricated implementation due to the robustness of reproducilibility of fabricated structures in silicon.
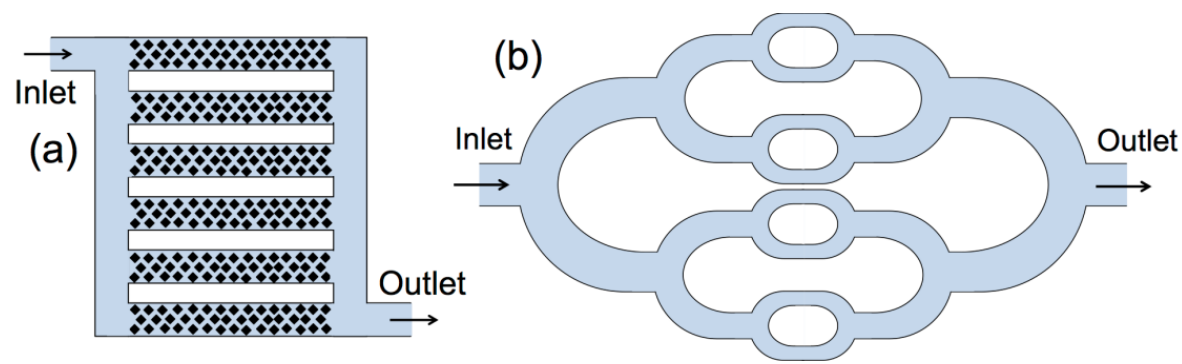

Figure 6. Different designs (a) parallel channel filled with pillars (b) branched parallel channels.

The design of the continuous-flow microchannel with integrated pillar arrays (Fig. 6(a)) was implemented with modifications from a previously reported design. ${ }^{19}$ A drawback of the continuous-flow design for the Nanopill would be its large hydraulic resistance due to a long serpentine channel with multiple pillars, needing a high power consumption micropump. ${ }^{19}$ Another approach to increase the microchannel surface area is with branched parallel channels (Fig. 6(b)), which has a reduced hydraulic resistance compared to the pillar array structure and requires less pressure to achieve the required flow rates. ${ }^{45}$ We have designed and implemented both parallel pillar array and parallel channel chips considering both lab-scale and Nanopill compatible design.

Table 2. Calculated surface area from full capture/elution MBD chip-hm-DNA assay.

\begin{tabular}{|c|c|}
\hline Channel Type & $\begin{array}{c}\text { Surface area } \\
\mathrm{mm}^{2}\end{array}$ \\
\hline Branched channels & 12 \\
\hline Parallel channels with pillars & 170 \\
\hline
\end{tabular}

The pillars are $50 \mu \mathrm{m}$ in height and the pillars have a square geometry $(10 \mu \mathrm{m}$ on a side) rotated $45^{\circ}$ with respect to the inlet flow stream in order to maximize the flow velocity for efficient sample elution. The surface area of the parallel 
channel design with pillars is $14 \times$ larger than the branched parallel channel design. The branched channel design with its large surface area has been used for characterizing the entire assay for a lab-scale demonstration. Branched parallel channel design has been considered as well for low-pressure situation since Nanopill has been the background motivation. Both design have been implemented and fabricated, however, only results from the pillar designs are presented in this chapter.

\subsubsection{SPR characterization of MBD assay}

A surface plasmon resonance (SPR) biosensor was used to characterize the MBD protein based hm-DNA capture/elution assay, shown in Fig. 7. A real-time sensorgram trace is first measured that indicates the conjugation of each moiety of the MUAM-biotin-SA-biotin-MBD2b sandwich complex that was previously described.

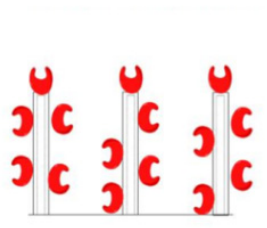

MBD2b-pillar array
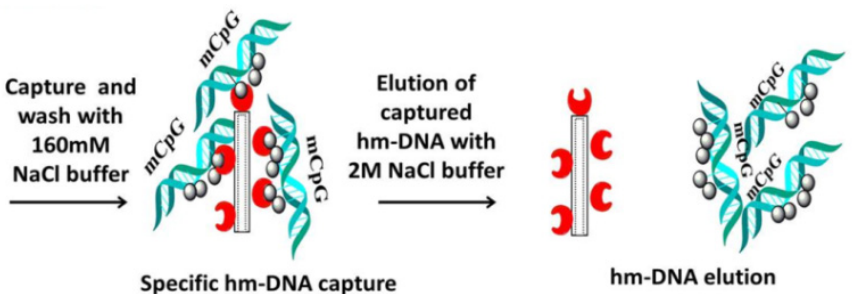

hm-DNA elution

Figure 7. Schematic showing the capture and elution protocol of hm-DNA from an MBD2b surface.

The real time capture and elution sequences are demonstrated using the SPR experiments shown in Fig. 8 and Fig. 9. The MUAM treated gold SPR disks are terminated in amine-end groups, and biotinylated with biotin-NHS. Starting with a biotinylated gold surface, the SA moiety is introduced to the surface at $t=180 \mathrm{~s}$, which saturates in about $150 \mathrm{~s}$, as shown in Fig. 8(a). The MBD2b protein at 35 $\mu \mathrm{g} \mathrm{mL}^{-1}$ is injected at $t=500 \mathrm{~s}$ (Fig. 8(a)) and washed with buffer $t=625 \mathrm{~s}$, and the baseline does not go back to the original, as there is binding of MBD2bbiotin complex on the SA layer. The MBD2b protein is used as a dilution from the commercial stock, and not prepared by reconstituting dry protein with same buffers as SA and hm-DNA, which results in a bulk refractive index change. The hm-DNA injection in Fig. 8(a) starts at $t=800 \mathrm{~s}$ on the MBD2b surface giving rise to a binding curve, showing no dissociation when washed with buffer at $t=900 \mathrm{~s}$. The top left inset in Fig. 8(a) is a gold surface not treated with MBD2b 
proteins, and therefore does not bind hm-DNA.

The biotin-SA binding is very strong and saturates the surface due to the small dissociation as observed during the washing step at $t=400 \mathrm{~s}$ (Fig. 8(b)). Non-hm-DNA injection on a separate MBD2b surface in Fig. 8(b) at $t=1150 \mathrm{~s}$ does not show strong binding and gets washed away with buffer at $t=1300 \mathrm{~s}$. A bare gold surface, which is not biotinylated, does not bind anything, as shown in the red trace in Fig. 8(b). Figs. 8(a) and 8(b) demonstrate the specificity of the MBD2b proteins for hm-DNA.
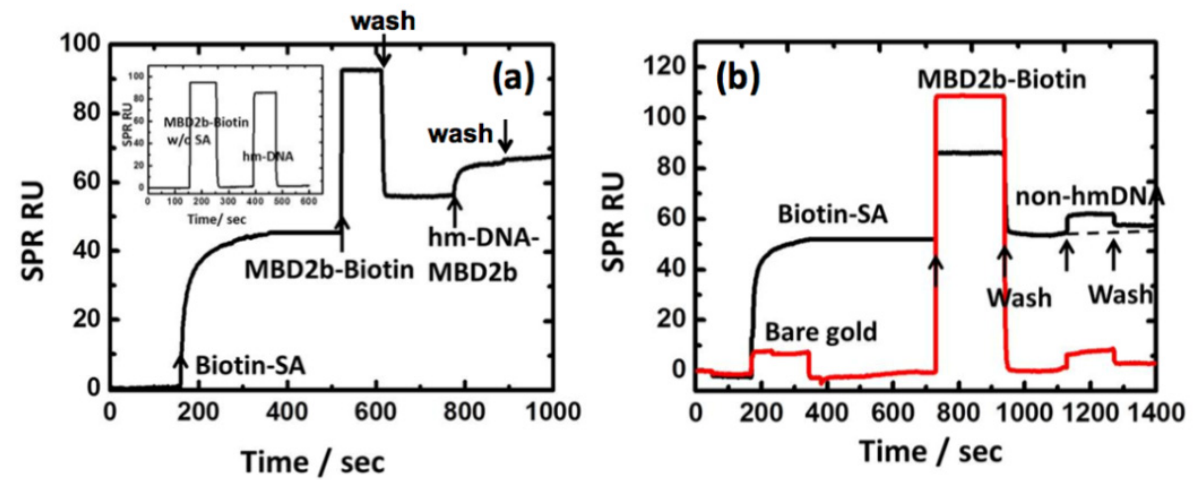

Figure 8. Real-time SPR sensorgrams with measured signals in instrument response unit (RU). (a) monitoring of the biotin-SA/MBD2b-biotin complex conjugation and subsequent binding of hm-DNA (b) selectivity of non-hm-DNA that is minimally hybridized with the MBD2b protein.

Figs. 9(a) and 9(b) demonstrate the capture and elution protocol of hm-DNA from the MUAM-biotin-SA-biotin-MBD2b surface. Fig. 9(a) shows preparation of the gold surface with the MBD2b protein. Using the MBD2b surface prepared in Fig. 9(a) hm-DNA prepared in $1 \times$ wash buffer $(160 \mathrm{mM} \mathrm{NaCl})$ is injected at $t=200 \mathrm{~s}$, followed by washing with wash buffer $t=380 \mathrm{~s}$, (Fig. 9(b)). There is a small amount of dissociation in elution buffer $(2 \mathrm{M} \mathrm{NaCl})$ injected at $t=580 \mathrm{~s}$, followed by washing with the wash buffer, where the baseline returns to the prehm-DNA injection, confirming that captured hm-DNA has been removed. The elution process is seen as a bulk refractive index change due to the difference in the buffer composition from the wash buffer. 

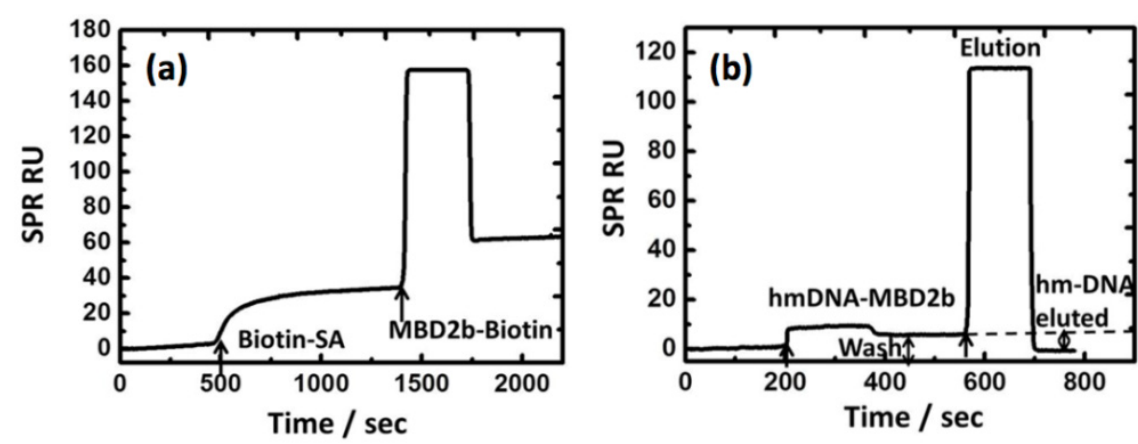

Figure 9. (a) MBD protein attachment (b) capture and elution of hm-DNA.

\subsubsection{Microfluidic chip fabrication}

We have designed and implemented a pillar array ${ }^{19}$ with square pillars $(10 \mu \mathrm{m}$ on a side) that are rotated $45^{\circ}$, with respect to the horizontal axis and and spaced $10 \mu \mathrm{m}$ apart. A commercial finite element simulator (Multiphysics, Comsol) was used to simulate the flow velocity in both structures, as shown in Fig. 10(a) and Fig. 10(b).
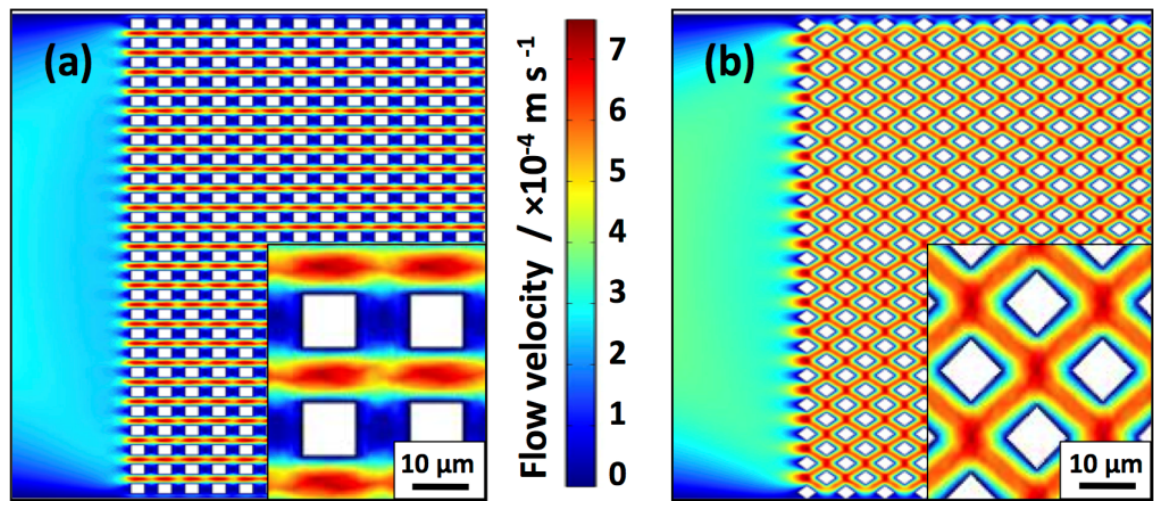

Figure 10. Finite element simulation of flow velocity in the different pillar designs (a) non-rotated pillars (b) pillar rotation $45^{\circ}$ to the direction of flow.

In case of the non-rotated (Fig. 10(a)) pillars spaced $10 \mu \mathrm{m}$ apart, there is a dead volume (blue regions) around the pillars, compared to the rotated $\left(45^{\circ}\right)$ pillar design (Fig. 10(b)) with uniform flow around the pillars (red regions). A flow velocity of $\sim 0.7 \mathrm{~mm} \mathrm{~s}^{-14}$ was calculated with the finite element simulations, requiring a pressure of $\Delta P=1$ bar applied across the microchannel, for enhanced hm-DNA elution from the surfaces. The branched parallel channels ${ }^{45}$ was also evaluated. 

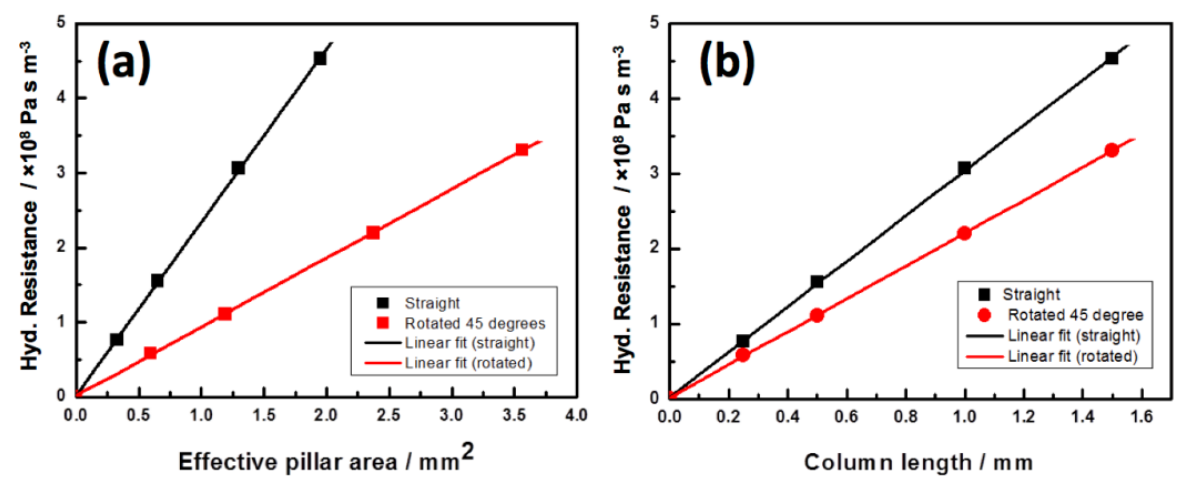

Figure 11. Comparison of hydraulic resistance for straight and rotated pillar orientation (a) with regard to effective pillar area and (b) column length.

The calculated hydraulic resistance for a rotated pillar and straight pillar is compared, for effective pillar area (Fig. 11(a)) and column length (Fig. 11(b)), and is always less in case of pillars with rotated geometry. Silicon-glass microchips with parallel $5 \mathrm{~mm}$ channels branched out at a lowest dimension of $10 \mu \mathrm{m}$, and $8 \mathrm{~mm}$ parallel channels $250 \mu \mathrm{m}$ width filled with $10 \mu \mathrm{m}$ square pillars spaced $10 \mu \mathrm{m}$ apart in array rotated by $45^{\circ}$ to the direction of flow were fabricated.

Figs. 12(a) and 12(b) show scanning electron micrograph (SEM) images of the two microchannel chip designs that are etched in silicon. The channels were etched to a depth of $50 \mu \mathrm{m}$ using a deep reactive-ion etching, which was previously optimized for a similar silicon etching load. The inset in
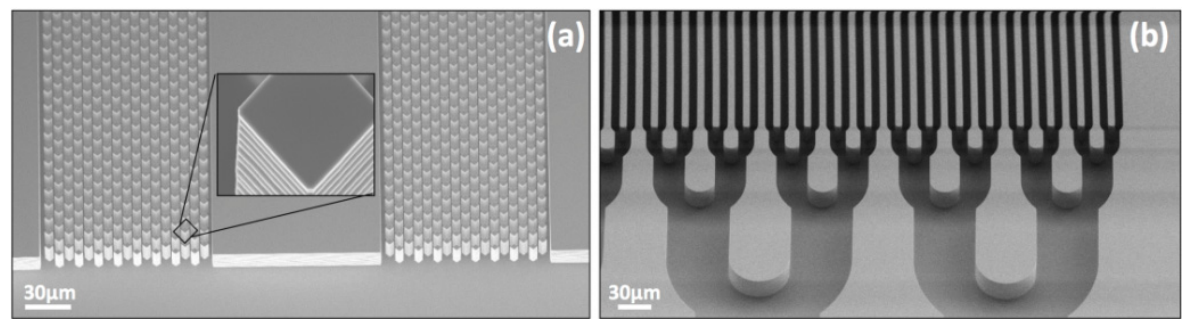

Figure 12. (a) SEM of pillar array (b) SEM of parallel channels.

Fig. 12(a) shows a single pillar with the scalloped pillar side wall due to etch and passivation cycles of the Bosch etching process. Fig. 13(a) and Fig. 13(b) are the optical images showing the microfabricated glass-silicon chips with inlet and outlet holes aligned to the microchannels, as used for the MBD assay experiments. 

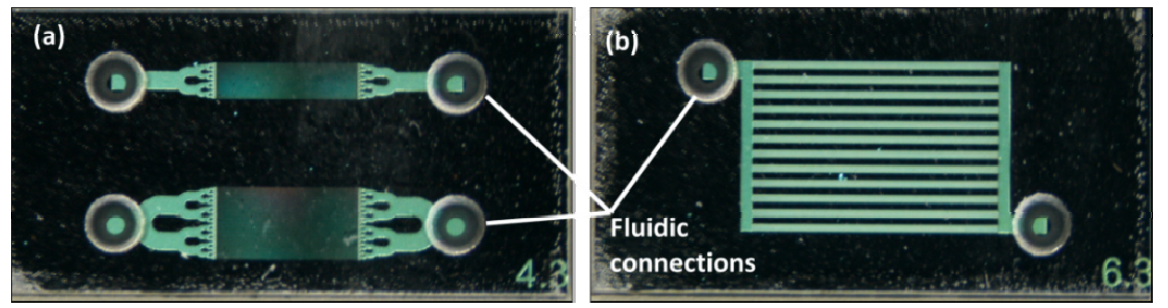

Figure 13. Optical images of glass-silicon bonded chips with inlet and outlet holes for (a) bifurcated parallel channels and (b) straight parallel channels with pillar array.

\subsubsection{MBD chip assay}

Fluorescence imaging due to SA-Alexafluor488 has been used, which binds to available MBD2b biotin sites as shown in Fig. 14(a) and Fig. 14(b). Fig. 14(a) demonstrates attachment of the MBD2b protein to the biotinylated surface where SA-Alexafluor brightly binds to available biotin from the MBD2b protein surface. Fig. 14(b) is an image of a control surface with an intial sandwich layer of biotin-SA without the MBD2b protein, followed by SA-488, which shows minimal fluorescence that is most likely due to nearly complete biotin binding to the APTES from the high SA concentration $(1 \mu \mathrm{M})$.
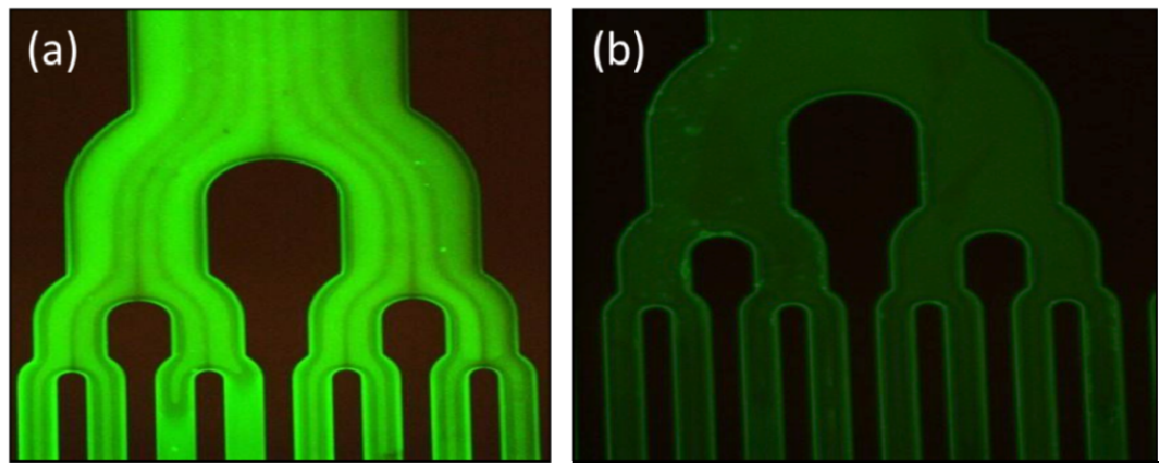

Figure 14. AlexaFluor488-SA used for surface imaging (a) and (b) MBD attachment to the $\mathrm{SiO}_{2}$ in parallel channels (c) MBD attachment to $\mathrm{SiO}_{2}$ pillar array (d) control (no-MBD).

Table 3 lists capture/elution results quantified using the complete assay protocol and fluorescence spectroscopy. Calibration plots of fluorescence intensity variations as a function of hm-DNA concentrations were measured and used for all quantification experiments (Fig. 3). For input concentrations of $1 \mathrm{ng} \mathrm{mL} \mathrm{m}^{-1}$, 
and lower, the capture/elution assay functions with high efficiency $\sim 84 \%$, is obtained. The assay appears to be getting saturated with about $4 \mathrm{ng}$ of DNA at higher concentrations near $100 \mathrm{ng} \mathrm{mL}^{-1}$.

Table 3. Measured average input and output capture/elution concentrations from the MBD-chip-hmDNA assay, with $1 \mu \mathrm{L}$ elution volume.

\begin{tabular}{|c|c|}
\hline $\begin{array}{c}\text { Input } \\
\text { concentration } \\
\mathrm{ng} \mathrm{mL}^{-1}\end{array}$ & $\begin{array}{c}\text { Output } \\
\text { concentration } \\
\mathrm{ng} \mathrm{mL}^{-1}\end{array}$ \\
\hline 1 & 0.9 \\
\hline 123 & 4 \\
\hline 200 & 3.8 \\
\hline
\end{tabular}

From these measurements, and considering that the total chip area for the combined design of parallel channels with pillars is $1.7 \mathrm{~cm}^{2}$, the protein attachment density is approximately $3 \times 10^{10} \mathrm{MBD} 2 \mathrm{~b}$ molecules $\mathrm{cm}^{-2}$ (assuming $100 \%$ capture efficiency), which is reasonable considering the mass of the MBD2b protein. ${ }^{50}$ The assay results show a linear capture/elution relationship for concentrations $<1 \mathrm{ng} \mathrm{mL} \mathrm{m}^{-1}$, as shown in Fig. 15 .

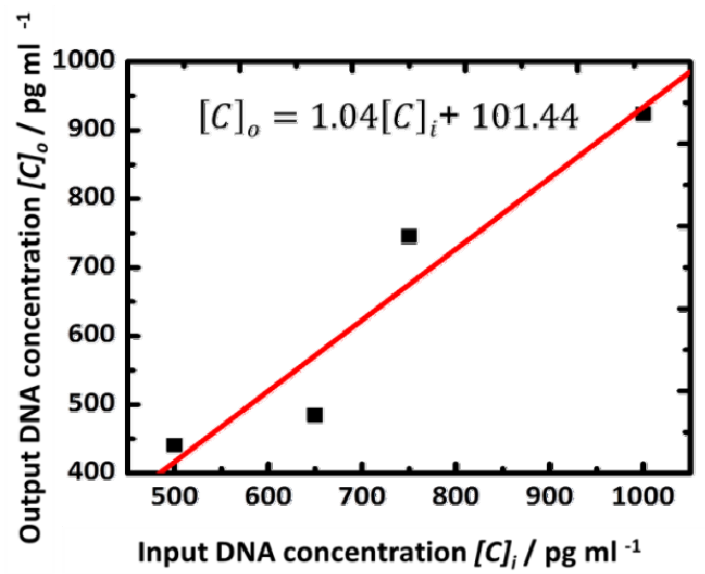

Figure 15. Capture and elution profile of MBD-chip-hm-DNA assay.

We have further used the MBD-assay to optimize the concentration capability for hm-DNA using the MBD glass-silicon chip. Table 4 lists the small volume elution of hm-DNA using the same assay for hm-DNA at a concentration of $1 \mathrm{ng} \mathrm{mL}{ }^{-1}$. We have tried three elution volumes of $25 \mu \mathrm{L}, 50$ $\mu \mathrm{L}$, and $100 \mu \mathrm{L}$, respectively, followed by ethanol precipitation and 
quantification with pico-green fluorescence assay. We observed that at elution volumes higher than $100 \mu \mathrm{L}$, the efficiency of elution is obviously improved, but compromising on the concentration factor. The efficiency of elution decreased to $71 \%$ with smaller elution volume of $25 \mu \mathrm{L}$. A $50 \mu \mathrm{L}$ elution volume showed an efficiency of $90 \%$, which increased only marginally to $92 \%$ with a $100 \mu \mathrm{L}$ elution volume. A $28 \times$ enrichment of input hm-DNA with $1 \mathrm{ng} \mathrm{mL}-1$ concentration and standard deviation of $1.7 \mathrm{ng} \mathrm{mL} \mathrm{m}^{-1}$ were obtained for the lowest elution volume of $25 \mu \mathrm{L}(\mathrm{n}=3)$. The elution efficiency at $100 \mu \mathrm{L}$ is $92 \%$, which is higher than the large volume $1 \mathrm{~mL}$ elution experiments of Table 3. Large elution volume $(1 \mathrm{~mL})$ post processing with ethanol precipitation could be the reason of loss of the recovered DNA. A small volume control run with nonhm-DNA, at $1 \mathrm{ng} \mathrm{mL} \mathrm{mL}^{-1}$ with a $200 \mu \mathrm{L}$ elution volume was investigated and resulted in $32 \%$ capture of the control non-hm-DNA. MBD2b proteins can discriminate hm-DNA from non-hm-DNA, as we have previously confirmed with SPR in Fig. 8(a) and Fig. 8(b), and we obtain a value for that as well. The efficiency was calculated as a ratio of the output concentration in the small volume eluent to the input concentration, concentrated in the small volume.

Table 4. Measured results from small volume capture-elution MBD-chip-hm-DNA assay with $1 \mathrm{~mL}$ load volume.

\begin{tabular}{|c|c|c|c|}
\hline $\begin{array}{c}\text { hm-DNA } \\
\mathrm{ng} \mathrm{mL} \mathrm{m}^{-1}\end{array}$ & $\begin{array}{c}\text { Eluted volume } \\
\mu \mathrm{L}\end{array}$ & $\begin{array}{c}\text { Eluted } \\
\text { hm-DNA } \\
\mathrm{ng} \mathrm{mL}^{-1}\end{array}$ & $\begin{array}{c}\text { Efficiency } \\
\%\end{array}$ \\
\hline 1 & 25 & $28 \pm 2$ & 71 \\
\hline 1 & 50 & $18 \pm 5$ & 90 \\
\hline 1 & 100 & $9 \pm 2$ & 92 \\
\hline
\end{tabular}

\subsection{Conclusion}

We have presented a small volume microfluidic chip that is capable of capturing low concentrations of hm-DNA $\left(<1 \mathrm{ng} \mathrm{mL}^{-1}\right)$ with a capture/elution efficiency of $84 \%$. All steps of the assay have been qualitatively characterized using a realtime SPR biosensor. The assay has been quantitatively characterized using calibrated fluorescence spectroscopy. A $28 \times$ output enrichment of input concentration of $1 \mathrm{ng} \mathrm{mL} \mathrm{mL}^{-1} \mathrm{hm}-\mathrm{DNA}$ was obtained in a $25 \mu \mathrm{L}$ elution volume with a std. deviation $1.7 \mathrm{ng} \mathrm{mL}^{-1}$ with the silicon-based MBD chip assay. The control DNA is captured 30\% when eluted with large volume of $200 \mu \mathrm{L}$, which for a $25 \mu \mathrm{L}$ assay can be expected to be smaller, which if minimized would 
improve the assay further. The capture and elution of hm-DNA on MBD surfaces is based on electrostatic effects as varying ionic strength of $\mathrm{NaCl}$ from $160 \mathrm{mM}$ to $2 \mathrm{M}$ is key to the binding and elution and use of multiple-MBDrecombinant protein might decrease the non-specific binding of non-hm-DNA considerably. 


\section{References}

1. A. Manz, N. Graber and H. M. Widmer, Sensors and Actuators: B. Chemical, 1990, 1, 244-248.

2. J. Comley, Drug Discovery World, 2012, 13, 23-45.

3. K. Adler, J. K. Moore, G. Filippov, S. Wu, J. Carmichael and M. Schermer, Journal of Molecular Diagnostics, 2011, 13, 614-620.

4. W. Pan, W. Chen and X. Jiang, Analytical Chemistry, 2010, 82, 3974-3976.

5. J. Hughes and A. E. Herr, Proceedings of the National Academy of Sciences of the United States of America, 2012, 109, 21450-21455.

6. G. Cooney, D. Sipes, N. Thakore, R. Holmberg and P. Belgrader, Biomedical Microdevices, 2012, 14, 45-53.

7. J. Rupp, M. Schmidt, S. Münch, M. Cavalar, U. Steller, J. Steigert, M. Stumber, C. Dorrer, P. Rothacher, R. Zengerle and M. Daub, Lab Chip, 2012, 12, 1384-1388.

8. O. Y. F. Henry and C. K. O'Sullivan, Trends in Analytical Chemistry, 2012, 33, 9-22.

9. S. G. Bavykin, J. P. Akowski, V. M. Zakhariev, V. E. Barsky, A. N. Perov and A. D. Mirzabekov, Applied and Environmental Microbiology, 2001, 67, 922-928.

10. Zhang, J. Xu, W. Ma and W. Zheng, Biotechnology Advances, 2006, 24, 243-284.

11. J. Min, J. H. Kim, Y. Lee, K. Namkoong, H. C. Im, H. N. Kim, H. Y. Kim, N. Huh and Y. R. Kim, Lab Chip, 2011, 11, 259-265.

12. C. Giordano, E. R. Copeland and J. P. Landers, Electrophoresis, 2001, 22, 328-333.

13. T. L. Hawkins, T. O. Connor-Morin, A. Roy and C. Santillan, Nucleic Acids Research, 1994, 22, 4543-4544.

14. V.Lund, R. Schimd, D. Rickwood and E. Hornes, Nucleic Acids Research, 1988, 16, 10861-10880.

15. Ø. Olsvik, T. Popovic, E. Skjerve, K. S. Cudjoe, E. Hornes, J. Ugelstad and M. Uhlén, Clinical Microbiology Reviews, 1994, 7, 43-54.

16. K. J. Purdy, T. M. Embley, S. Takii and D. B. Nedwell, Applied and Environmental Microbiology, 1996, 62, 3905-3907.

17. Šafařík and M. Šafaříková, Journal of Chromatography B: Biomedical Sciences and Applications, 1999, 722, 33-53. 
18. H. Tian, A. F. R. Hühmer and J. P. Landers, Analytical Biochemistry, 2000, 283, 175-191.

19. N. C. Cady, S. Stelick and C. A. Batt, Biosensors and Bioelectronics, 2003, 19, 59-66.

20. Odeberg, Z. Yun, A. Sonnerborg, M. Uhlen and J. Lundeberg, Journal of Clinical Microbiology, 1995, 33, 1870-1874.

21. Adessi, G. Matton, G. Ayala, G. Turcatti, J. J. Mermod, P. Mayer and E. Kawashima, Nucleic Acids Research, 2000, 28.

22. S. Bronzeau and N. Pamme, Analytica Chimica Acta, 2008, 609, 105-112.

23. Verpoorte, Lab Chip, 2003, 60N.

24. G. Rashkovetsky, Y. V. Lyubarskaya, F. Foret, D. E. Hughes and B. L. Karger, Journal of Chromatography A, 1997, 781, 197-204.

25. A. Hayes, N. A. Polson, A. N. Phayre and A. A. Garcia, Analytical Chemistry, 2001, 73, 5896-5902.

26. J.-W. Choi, K. W. Oh, J. H. Thomas, W. R. Heineman, H. B. Halsall, J. H. Nevin, A. J. Helmicki, H. T. Henderson and C. H. Ahn, Lab Chip, 2002, 2, 27-30.

27. M. Miller, P. E. Sheehan, R. L. Edelstein, C. R. Tamanaha, L. Zhong, S. Bounnak, L. J. Whitman and R. J. Colton, Journal of Magnetism and Magnetic Materials, 2001, 225, 138-144.

28. Z. H. Fan, S. Mangru, R. Granzow, P. Heaney, W. Ho, Q. Dong and R. Kumar, Analytical Chemistry, 1999, 71, 4851-4859.

29. G. Jiang and D. J. Harrison, Analyst, 2000, 125, 2176-2179.

30. S. Kwakye and A. Baeumner, Analytical and Bioanalytical Chemistry, 2003, 376, 1062-1068.

31. L. Korecká, Z. Bílková, M. Holèapek, J. Královský, M. Beneš, J. Lenfeld, N. Minc, R. Cecal, J. L. Viovy and M. Przybylski, Journal of Chromatography B: Analytical Technologies in the Biomedical and Life Sciences, 2004, 808, 15-24.

32. V. I. Furdui and D. J. Harrison, Lab Chip, 2004, 4, 614-618.

33. V. I. Furdui, J. K. Kariuki and D. J. Harrison, Journal of Micromechanics and Microengineering, 2003, 13, S164-S170.

34. D. Tarn and N. Pamme, Expert Review of Molecular Diagnostics, 2011, 11, 711-720. 
35. L. C. Waters, S. C. Jacobson, N. Kroutchinina, J. Khandurina, R. S. Foote and J. M. Ramsey, Analytical Chemistry, 1998, 70, 158-162.

36. M. A. Burns, B. N. Johnson, S. N. Brahmasandra, K. Handique, J. R. Webster, M. Krishnan, T. S. Sammarco, P. M. Man, D. Jones, D. Heldsinger, C. H. Mastrangelo and D. T. Burke, Science, 1998, 282, 484487.

37. T. Woolley, D. Hadley, P. Landre, A. J. deMello, R. A. Mathies and M. A. Northrup, Analytical Chemistry, 1996, 68, 4081-4086.

38. R. C. Anderson, X. Su, G. J. Bogdan and J. Fenton, Nucleic Acids Research, 2000, 28, e60.

39. K. Yuen, L. J. Kricka, P. Fortina, N. J. Panaro, T. Sakazume and P. Wilding, Genome Research, 2001, 11, 405-412.

40. R. G. Blazej, P. Kumaresan and R. A. Mathies, Proceedings of the National Academy of Sciences of the United States of America, 2006, 103, 72407245.

41. Y. M. Dennis Lo, C. Rossa W K and K. C. Allen Chan, Clinical Applications of PCR, Totowa, N.J: Humana Press, 2006.

42. Methyl Miner handbook, Invitrogen.

43. K. A. Hagan, C. R. Reedy, M. L. Uchimoto, D. Basu, D. A. Engel and J. P. Landers, Lab Chip, 2011, 11, 957-961.

44. M. Zimmermann, E. Delamarche, M. Wolf and P. Hunziker, Biomedical Microdevices, 2005, 7, 99-110.

45. W. Cao, C. J. Easley, J. P. Ferrance and J. P. Landers, Analytical Chemistry, 2006, 78, 7222-7228.

46. J. Wen, L. A. Legendre, J. M. Bienvenue and J. P. Landers, Analytical Chemistry, 2008, 80, 6472-6479.

47. Y. Liu, N. C. Cady and C. A. Batt, Biomedical Microdevices, 2007, 9, 769776.

48. C. R. Reedy, K. A. Hagan, B. C. Strachan, J. J. Higginson, J. M. Bienvenue, S. A. Greenspoon, J. P. Ferrance and J. P. Landers, Analytical Chemistry, 2010, 82, 5669-5678.

49. C. Lui, N. C. Cady and C. A. Batt, Sensors, 2009, 9, 3713-3744.

50. W. Shu, E. D. Laue and A. A. Seshia, Biosensors and Bioelectronics, 2007, 22, 2003-2009. 
Chapter 4 


\section{Chapter 5}

\section{Microfluidic bio(chemical) analysis system with integrated silicon nanowire sensor arrays ${ }^{3}$}

In this chapter, we report an integrated label-free analysis platform for simultaneous fluid and electronic measurements together for detection on PNADNA binding in flow. The integrated set up is about characterizing the PNADNA binding on Si-NWs which would be eventually used in low power consuming Si-NW sensor cartridge for the Nanopill. This integrated microfluidic platform is suitable for a wide range of small volume chemical and biochemical analyses. The integrated platform uses a disposable analysis cartridge consisting of all-electrical Si-NW sensor arrays, for (bio)chemical detection, integrated with a small volume microfluidic flow-cell. Sample delivery in the integrated analytical instrument is performed with an automated multi-sample injection system that eliminates erroneous sensor responses from sample switching due to flow rate fluctuations and provides precise sample volumes. The sample transport to sensor surface is controlled to ensure that reaction-limited binding models can be used to estimate the biomolecular affinity. We describe the components of the integrated measurement system and its use for meaningful data acquisition for real-time biosensing applications. The efficacy of the integrated system is demonstrated by measuring sample $p H$ in flow conditions of sequentially injected sample buffers and subsequently used for real-time PNA-DNA (peptide nucleic acid) duplex hybridization measurements together with an estimation of the binding affinity.

\footnotetext{
${ }^{3}$ Adapted from: A. De, J. van Nieuwkastelle, E.T. Carlen, A. van den Berg, Integrated labelfree silicon nanowire sensor arrays for (bio)chemical analysis, Analyst, 2013, 138, 3221 3229.
} 


\subsection{Introduction}

Miniaturized analytical assays for the detection of biomolecular interactions at very low sample concentrations and volumes are becoming increasingly important for applications requiring high-throughput biomolecular analyses, such as genomics screening, protein assays for basic molecular biology research, pharmaceutical drug screening, and disease diagnostics. ${ }^{1}$ The most common method for biomolecular detection uses a reporter label, such as a fluorophore or radioisotope, chemically attached to the target analyte that indicates binding to a receptor molecule by measuring the emission signal of the reporter label. Currently, fluorescence-based detection of target-receptor biomolecular binding is most commonly used, which is well established with many commercially available high quantum yield fluorophores and highly sensitive detectors, however, the labeled approach suffers from the need to conjugate the label to the target and possible alterations in target-receptor interactions caused by conformational changes or steric hindrance induced by the label. ${ }^{2}$

There has been considerable effort over the last two decades to develop label-free measurement techniques that can directly record biomolecular complex binding events without significantly altering the native biomolecular conformation. Isothermal titration calorimetry and differential scanning calorimetry are truly label-free methods that can measure the thermodynamic binding properties in the native biomolecular environment. ${ }^{3}$ Although the calorimetric methods are extremely powerful, they are currently limited to large sample volumes and concentrations. Alternatively, the receptor molecule can be tethered directly to a sensor surface that can measure in real-time the change of surface properties as the target analyte binds to the receptors. Currently, many different surface-based label-free sensor systems are available that use different detection phenomena, such as surface plasmon resonance, optical interferometry, optical gratings, quartz crystal microbalance, and surface acoustic waves. ${ }^{4}$ Surface plasmon resonance sensors ${ }^{5}$ and surface plasmon resonance imaging sensors $^{6}$ are well-established label-free techniques, but they require sophisticated optical instrumentation for signal readout and have limited sensitivity, especially for small molecular weight molecules. Another promising technique uses electrochemical labels and biomolecular binding is measured with a conventional integrated circuit ${ }^{7}$. All electrical readout of biomolecular binding can also be realized with semiconductor transistors by measuring the 
intrinsic electronic biomolecular charge on the gate-oxide using a field-effect to modulate the device conductance. ${ }^{8,9}$

There has been a steady increase in reports of ultrasensitive biosensors over the past several years based on semiconductor nanowires. ${ }^{10}$ Onedimensional electrical semiconductor nanowire sensors are particularly important due to their suitability for large-scale, high-density integration and interfacing to conventional electronic systems, hence are attractive for low cost portable sensing systems. Silicon nanowire (Si-NW) field-effect biosensors have been reported extensively for the highly sensitive, label-free, and real time detection of biomolecular binding of DNA and proteins. ${ }^{11,12}$ The high detection sensitivity of silicon nanowire biosensors has been attributed to their large surface-to-volume ratio and the three-dimensional multi-gate structure; both contribute to the improved sensitivity compared to conventional planar devices. However, device and technology development have been limited to a small number of research laboratories typically relying on expensive nanolithography or specialized equipment and processes. We recently reported a new scalable silicon nanowire fabrication technology based on a combination of conventional microfabrication that does not require expensive nanolithography. ${ }^{13}$ The main advantage of our technology is that dense sensor arrays, with precisely controlled dimensions (down to $\sim 5 \mathrm{~nm}$ ) and atomically smooth surfaces can be manufactured in any conventional microfabrication cleanroom. Additionally, the top-down fabricated Si-NW biosensor chips can be easily integrated with a small volume microfluidic flow-cell.

The integrated microfluidic label-free analysis platform presented in this chapter uses a disposable analysis cartridge consisting of all-electrical SiNW sensor arrays, for (bio)chemical detection, integrated with a small volume microfluidic flow-cell. The low power consumption Si-NW (bio)chemical sensor cartridge has been developed for the Nanopill system.

\subsection{Experimental}

\subsubsection{Autosampler}

The core of the integrated analysis system consists of an automated multisample delivery subsystem that can deliver precise sample volumes to the integrated microfluidic analysis cartridge using regulated pressure sources. The autosampler is made by using a sample injection valve (Cheminert Nanovolume 
10-port valve, Valco Instruments Co. Inc.) with a regulated pressure source (MFCS-8C, Fluigent) and completed with sample loops made from polyetherether-ketone (PEEK) tubing (TPK 10×, Valco Instruments Co. Inc.). Fig. 1 shows a schematic diagram of the protocol for operating the autosampler. PEEK tubing attached to the inlet port was connected directly to the pressurized sample reservoir $\left(S R_{1}, S R_{2}\right.$ and $\left.S R_{3}\right)$, which are filled with $2 \mathrm{ml}$ sample reservoir tubes (T341-6TLST 2mL, Econo Lab) of sample buffers. Different valve positions are automatically set as switch position A or B during the injection procedure.
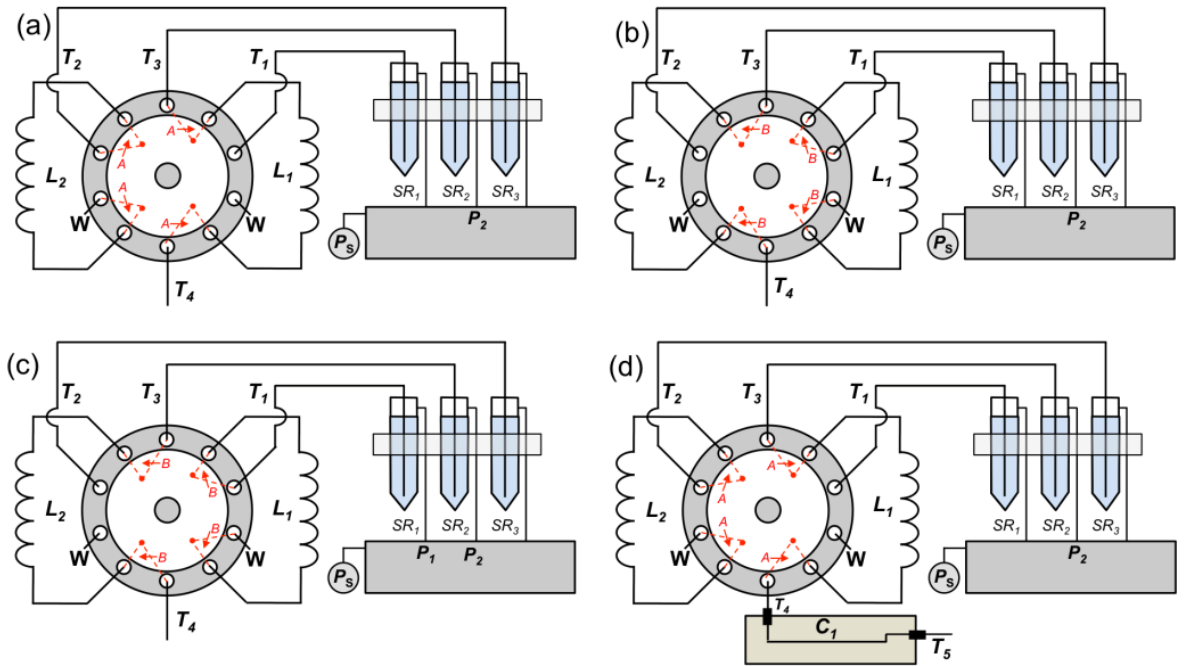

Figure 1. Automated sample injector protocol. (a) System purge with buffer, driving pressure through SR1, valve in position A (b) System purge with buffer, driving pressure through SR1, valve in position B (c) valve in position B, filling of loop L1, by applying pressure to SR1 (d) Injection of sample loop L1, valve position changed to position A and T4 connected to microchannel.

A sample plug injection from $S R_{1}$ is injected into the microfluidic flow-cell with buffer from $\mathrm{SR}_{2}$.

The protocol for the loading and injection of a single sample is described below:

Step 1 (Fig. 1(a) and Fig. 1(b)): System purge (microfluidic flow-cell not connected to $\left.T_{4}\right)$ :

- $\quad$ Purge sample loop $L_{1}$ with buffer $\left(S R_{2}, P_{2}=1\right.$ bar; $T_{3}+L_{1}+T_{4}$; Fig. 1(a), switch pos. A)

- $\quad$ Purge sample loop $L_{2}$ with buffer $\left(S R_{2}, P_{2}=1\right.$ bar; $T_{3}+L_{2}+T_{4}$; Fig. 1(b), switch pos. B)

Step 2 (Fig. 1(c), switch pos. B): Load sample 1 to loop $L_{1}$ : 
- $\quad$ Load sample loop $L_{1}$ with sample $1\left(S R_{1}, P_{1}=1\right.$ bar; $T_{1}+L_{1}$ to waste $\left.W\right)$

- Stop when sample appear at waste $\mathrm{W}$ outlet

Step 3 (Fig. 1d, switch pos. A): Injection of sample $1\left(S R_{1}\right)$ :

- Connect $T_{4}$ to microfluidic flow-cell

- Continue injecting buffer $\left(S R_{2}, P_{3}=1\right.$ bar $)$ through sample loop $L_{2}$ and microfluidic flow-cell $\left(T_{3}+L_{2}+T_{4}+C_{1}+T_{5}\right)$ using maximum drive pressure, i.e. $P_{2}=1$ bar

- When sensor output is stable inject sample $1\left(S R_{2}, P_{2} ; T_{3}+L_{1}+T_{4}+C_{1}+T_{5}\right)$

The tubing lengths and diameters $\left(T_{3}, T_{4}\right.$ and $\left.T_{5}\right)$ are determined by the range of the regulated pressure source $P_{2}$ and desired sample flow rate $Q$. The total equivalent hydraulic resistance $R_{\text {hyd }}$ and the volumetric flow rate $Q$ can be estimated with $Q=\Delta P / R_{\text {hyd }}$. The hydraulic resistance of a tube, or microchannel with circular cross-section is $R_{\mathrm{hyd}} \approx 8 \mu L / \pi a^{4}$, where $\mu$ is the dynamic viscosity of the sample ( $\mu \approx 10^{-3} \mathrm{~Pa}$ s for water), $L$ is the length, and $a$ is the radius of the cross-section of the hydraulic component. Table 1 shows typical hydraulic resistances for the various tubing and microchannel. The sample loops provide the capability to deliver a sample plug with volume from about $10 \mathrm{nl}$, volume of $T_{3}$ as described in Table 1. up to a larger volume that is dependent on the length of the sample loops.

Table 1. Hydraulic resistances of the different autosampler units.

\begin{tabular}{|c|c|c|c|c|}
\hline Tube & $\begin{array}{c}\phi \\
\mu \mathrm{m}\end{array}$ & $\begin{array}{l}2 \times a \\
\mu \mathrm{m}\end{array}$ & $\begin{array}{l}\boldsymbol{L} \\
\mathrm{m}\end{array}$ & $\begin{array}{c}\boldsymbol{R}_{\text {hyd }} \\
\mathrm{Pa} \mathrm{s} \mathrm{m}^{-3}\end{array}$ \\
\hline $\mathrm{T}_{3}\left(\mathrm{R}_{3}\right)$ & \multirow{3}{*}{360} & 50 & 0.2 & $1.2 \times 10^{15}$ \\
\hline $\mathrm{T}_{4}\left(\mathrm{R}_{4}\right)$ & & 150 & 3.1 & $2.4 \times 10^{14}$ \\
\hline $\mathrm{L}_{1}, \mathrm{~L}_{2}\left(\mathrm{R}_{1}, \mathrm{R}_{2}\right)$ & & 150 & 1.6 & $1.2 \times 10^{14}$ \\
\hline $\mathrm{C}\left(\mathrm{R}_{\mathrm{c}}\right)$ & - & 360 & 0.01 & $2.8 \times 10^{10}$ \\
\hline $\mathrm{T}_{5}\left(\mathrm{R}_{5}\right)$ & 700 & 500 & 0.03 & $1.9 \times 10^{10}$ \\
\hline
\end{tabular}

The sample loop volume, sample flow rate, and experiment duration have been used to determine the length and inner diameter of the PEEK tubing, $T_{1}, T_{2}, T_{3}$, $T_{4}$ and $T_{5}$ and source pressures $P_{1}-P_{3}$, as shown in Table 1 . 


\subsubsection{Silicon nanowires}

Triangular Si-NWs have been fabricated with the previously reported method developed by our group that uses conventional microlithography, wet anisotropic etching and size reduction, oxidation, and dry etching. ${ }^{13}$ Briefly SiNW sensors were fabricated from silicon-on-insulator (SOI) wafers (G4P-00502 , Soitec) using anisotropic wet etching (5\% tetra methyl ammonium hydroxide) of the silicon device layer. The advantage of our technique is that dense arrays of Si-NWs, with precisely controlled dimensions and atomically smooth surfaces, are directly fabricated with thick $(200 \mathrm{~nm})$ microscale electrical contact regions from a continuous layer of single crystal silicon. The thick silicon contact regions result in reduced contact resistance compared to ultrathin silicon layers. This fabrication technology is capable of producing high-density Si-NW arrays with device diameters down to $10 \mathrm{~nm}$ and $50 \mathrm{~nm}$ pitch with the use of high resolution patterning methods to define the initial masking layer. All sensors are boron doped $\left(N_{a} \sim 10^{18} \mathrm{~cm}^{-3}\right)$ with heights $a=140$ $\mathrm{nm}$ or $100 \mathrm{~nm}$, measured with atomic force microscopy (AFM). Boron doping was done with conventional ion implantation and $\mathrm{N}_{2}$ anneal at $1000{ }^{\circ} \mathrm{C}$ for 30 min for dopant drive-in and activation. The aluminum electrical contacts have been annealed at $450{ }^{\circ} \mathrm{C}$ for $20 \mathrm{~min}$ in an $\mathrm{H}_{2} / \mathrm{N}_{2}$ forming gas to reduce interface effects and reduce metal-silicon contact resistance, which have ohmic characteristics. The Si-NW chips were then encapsulated with a $2.5 \mu \mathrm{m}$ thick polyimide (PI) (annealed at $350{ }^{\circ} \mathrm{C}$ for $1 \mathrm{~h}$ ) electrical isolation layer that has lithographically patterned sensing windows over the Si-NW sensor regions and electrical contact regions. A $15 \mathrm{~nm}$ thick silicon dioxide gate-oxide layer was used for $\mathrm{pH}$ measurements and 1-2 $\mathrm{nm}$ thick silicon dioxide gate-oxide layer was used for DNA hybridization experiments. Fig. 2(a) shows high resolution scanning electron microscopy (SEM) and high-resolution transmission electron microscopy (TEM) images that are representative of Si-NWs fabricated with this technology. Fig. 2(b)-(d) shows optical microscopy images of the different device types used for experiments in this article. Fig. 2(e) shows an example of the Si-NW chip with an array of 2-wire Si-NW sensors and the inset shows an example of a sensing window patterned in the PI isolation layer. 


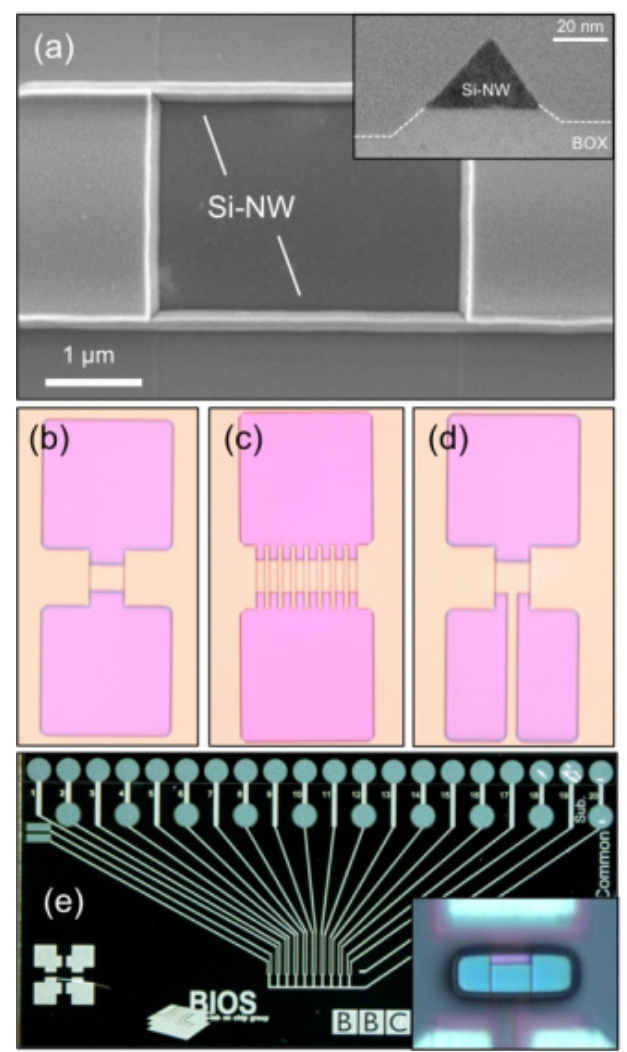

Figure 2. Microscopy images of fabricated Si-NWs. (a) High-resolution SEM image of 2-wire Si-NW device. Inset: High-resolution TEM Si-NW cross-section (b-d) Optical images (b) 2-wire Si-NW device (c) 16-wire Si-NW device (d) Differential Si-NW device with split source contact (e) Si-NW sensor chip $(1 \mathrm{~cm} \times 2 \mathrm{~cm})$ encapsulated with PI layer. Inset: patterned sensing windows in PI exposing the SiNW sensors.

\subsubsection{Nanoscale imaging and metrology}

\subsubsection{High resolution TEM sample preparation and imaging}

Si-NW samples were prepared by depositing a $100 \mathrm{~nm}$ thick silicon nitride protection layer onto the upper surface. The sample slices we prepared in a dualbeam focused ion beam (FEI Tecnai G2 F20 X-Twin FEG TEM, Maser Engineering, Enschede, The Netherlands) and transferred to a TEM imaging grid. TEM imaging (FEI 3D-Strata DB-FIB FEG, Maser Engineering, Enschede, The Netherlands) was operated at $200 \mathrm{kV}$ acceleration voltage. The TEM image is seen in Fig. 2(a)(inset). 


\subsubsection{High resolution SEM sample preparation and imaging}

Si-NW samples were coated with a $\mathrm{Au} / \mathrm{Pd}$ thin film prior to imaging to reduce charging of the top oxide surface. SEM imaging (LEO 1550, Zeiss) was performed at acceleration voltages ranging from $2 \mathrm{kV}$ to $10 \mathrm{kV}$.

\subsubsection{AFM imaging}

A Digital Instruments Dimension 3100 was used to capture all AFM images. All AFM images performed in tapping mode with ultra-sharp (average tip diameter $\sim 2 \mathrm{~nm}$ ) single crystal silicon tips (SSH-NCH-10, NanoandMore, $\mathrm{GmbH}$ ).

\subsubsection{Microfluidic flow-cell}

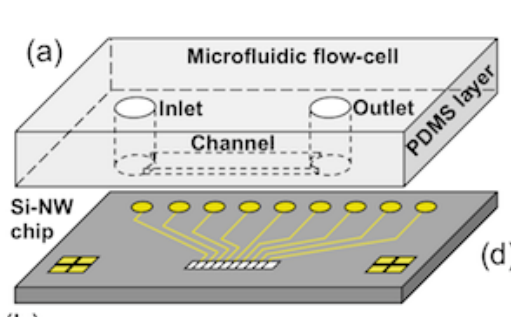

\section{(b)}

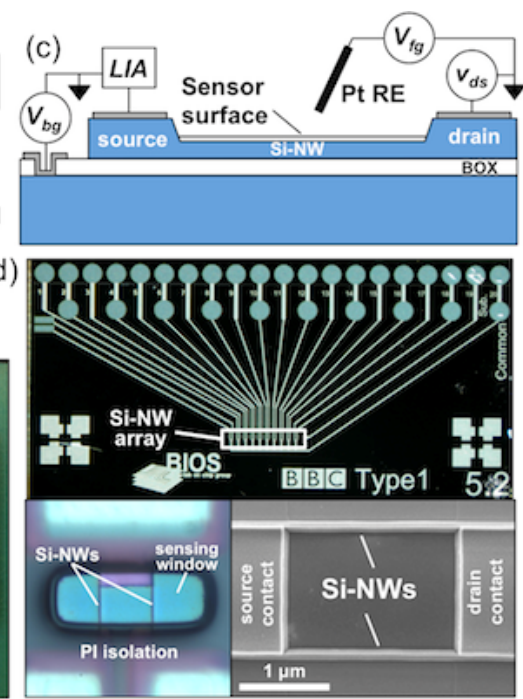

Figure 3. Integrated microfluidic biosensor cartridge consisting of Si-NW biosensor chip integrated with microfluidic flow-cell. (a) Schematic of Si-NW sensor chip and PDMS microfluidic flow-cell. (b) Optical microscopy image of implemented integrated microfluidic biosensor cartridge with Si-NW biosensor chip attached and wire bonded to custom circuit board, and bonded microfluidic flow-cell. (c) Length cross-section schematic of Si-NW biosensor with back-gate $V_{\mathrm{bg}}$, front-gate $V_{\mathrm{fg}}$, and drain-source $v_{\mathrm{ds}}$ bias voltages. Liquid gating of the Si-NW is controlled with a $\mathrm{Pt}$ reference electrode (RE) connected to $V_{\text {fg. }}$. A lock-in amplifier (LIA) measures the sensor current. (d) Microscopy images of fabricated Si-NWs. Upper: Si-NW sensor chip $(1 \mathrm{~cm} \times 2 \mathrm{~cm})$. Lower left: $\mathrm{Si}-\mathrm{NW}$ sensor encapsulated with PI layer and patterned sensing window. Lower right: High-resolution SEM image of 2-wire SiNW sensor.

The microfluidic chip is made from casted polydimethylsiloxane (PDMS) on a 
silicon mold using a 10:1 pre-polymer (Sylgard 184) to curing agent (Dow Corning) mixture. Inlet and outlet holes were formed in the microfluidic flowcell with a punch tool ( $\phi 0.5 \mathrm{~mm}$ Unicore, Harris). Following surface cleaning of the Si-NW sensors, and subsequent modification with probe biomolecules as required, the $\mathrm{Si}-\mathrm{NW}$ sensor chip is attached and wire bonded to a custom-made circuit board. The assembled Si-NW sensor chip is then aligned and clamped to the PDMS microfluidic chip, which is simple and non-destructive to the sensor surfaces and probe biomolecules on the sensor surfaces. Fig. 3(a) shows a schematic diagram of the integrated microfluidic biosensor analysis cartridge. The inlet and outlet holes of the PDMS microfluidic channel are connected to the sample injection valve with PEEK tubing. Fig. 3(c) shows the schematic for Si-NW electrical measurement with a Pt front gate electrode. For experiments presented in this article the PDMS microfluidic channel is $1 \mathrm{~cm}$ in length and $360 \mu \mathrm{m}$ in diameter. A platinum wire (267171, Sigma-Aldrich) pseudo-reference electrode is inserted directly into the microfluidic channel. Fig. 3(b) shows an example of an integrated microfluidic analysis cartridge with inlet and outlet holes that are interfaced directly to the PEEK tubing. The analysis cartridge is mounted and wire bonded to a custom-made circuit board.

\subsubsection{Sensor surface preparation}

Following encapsulation of the Si-NW sensor chip with the PI isolation layer, and prior to sensing experiments, the gate-oxide surfaces are cleaned with a UVozone treatment (UV/Ozone ProCleaner Plus, Bioforce Nanosciences) for $3 \mathrm{~min}$. Gate-oxide surface cleaning with UV ozone before each measurement is essential for proper device operation. The electrical operation of the Si-NWs is optimal with short ozone treatment times; however, prolonged exposure to UV can degrade performance through the creation of surface states at the gate-oxide silicon interface. ${ }^{14}$ Exposure continuously for more than 15 min resulted in nonfunctional devices, which is especially problematic for NWs as they typically have a large surface-to-volume ratio and are extremely sensitive to interface properties. The device operation degradation, as a result of surface states, is observed by a continuous reduction of conductance to zero, which typically occurs within seconds. This kind of radiation damage can be reduced with low temperature annealing (e.g. $\left.350^{\circ} \mathrm{C}\right) .{ }^{15}$ 


\subsubsection{1 pH sensor}

The surface potential $\psi_{\mathrm{o}}$ of the Si-NW gate-oxide is dependent on the $\mathrm{pH}$ of the sample solution and is obtained through the titration of the gate-oxide in the presence of a supporting electrolyte. The Si-NW sensor surface is first cleaned with UV ozone, as previously described, and then the Si-NW sensor chip is attached and wire bonded to a custom circuit board and clamp-bonded with the PDMS microfluidic chip. The platinum reference electrode is inserted into the microfluidic flow channel. Prior to solution measurements, a $0.1 \mathrm{M} \mathrm{NaCl}$ supporting electrolyte with $0.01 \mathrm{M}$ universal buffer mixture (UBM: $0.01 \mathrm{M}$ citric acid, $0.01 \mathrm{M}$ phosphoric acid, and $0.02 \mathrm{M}$ boric acid) solution at $p H 4$ was flowed from $S R_{1}$ through the microchannel for 20 minutes to equilibrate the sensor surface. ${ }^{16}$ The $p H 7$ and 10 solutions were realized by titrating the starting solution with a $1 \mathrm{M} \mathrm{NaOH}$ solution.

\subsubsection{DNA hybridization}

The surface potential $\psi_{0}$ of the Si-NW gate-oxide is dependent on the intrinsic electronic charge of biomolecules, such as DNA hybridized with complementary probes attached near the sensor surface.

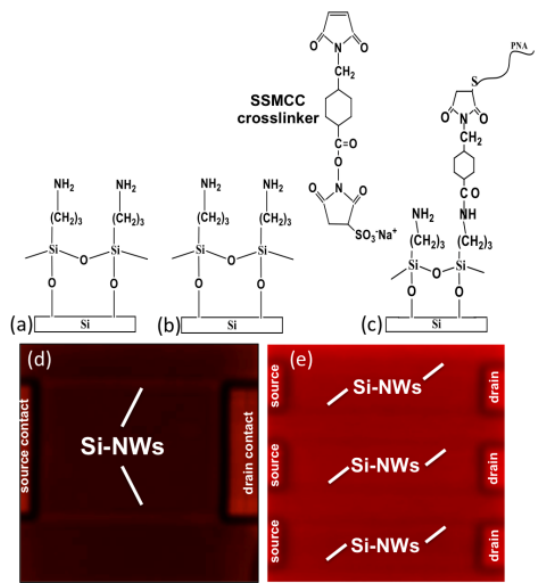

Figure 4. PNA attachment to the Si-NW gate-oxide surface. (a-c) PNA attachment scheme. (d) Negative control incubated with PNA-Cy5 conjugate. A single Si-NW sensor pair is shown. (e) Positive control incubated with PNA-Cy5. Three Si-NW sensor pairs are shown.

A sensor response is obtained by measuring the device conductance change, which is dependent on surface potential changes as target biomolecules 
accumulate on the gate-oxide surface in the presence of an aqueous buffer. Following cleaning of the gate-oxide surfaces with UV ozone, amine functional groups are conjugated to the gate-oxide sensor surface using a 3-amino propyl triethoxy silane (APTES) monolayer. Si-NWs were incubated with APTES in $95 \%$ ethanol and $5 \%$ deionized water for $1 \mathrm{hr}$. The surface was then washed with ethanol and followed by a cross-linking heat treatment at $110{ }^{\circ} \mathrm{C}$ for $15 \mathrm{~min}$ (Fig. 4(a)). The amine-functionalized surface was activated with a $5 \mathrm{mM}$ sulfo succinimide maleimide cyclohexane carboxylate cross-linker (SSMCC, PierceThermo Scientific) in a $100 \mathrm{mM}$ triethanolamine buffer with $p H 7.2$ for $1 \mathrm{hr}$ (Fig. 4(b)). The surface is then incubated in $1 \mu \mathrm{M}$ thiolated peptide nucleic acid (PNA) in the same buffer for 12 hrs (Fig. 4(c)). A synthetic 15-mer thiolated PNA probe was used for all hybridization experiments with sequence: Cys-TGTACA-TCA-CAA-CTA- $\mathrm{NH}_{2}$. The complementary $(\mathrm{MO})$ deoxyribonucleic acid (DNA) sequence is: 5'-TAG-TTG-TGA-TGT-ACA-3' (Eurogentec). The noncomplementary (NC) DNA sequence is: 5'-ATC-AAC-ACT-ACA-TGT-3' (Eurogentec). DNA-PNA duplex hybridization on the gate-oxide of Si-NWs was performed in a $1 \mathrm{mM}$ phosphate buffer with $p H$ 7.4. A fluorophore (Cy5) labeled PNA probe (Eurogentec) was used as a control to confirm conjugation of the PNA probe to the gate-oxide sensor surface. We used a $60 \mu 1$ volume PDMS incubation well clamped to the Si-NW chip placed in a humidity chamber for all surface chemistry steps. The efficacy of the PNA probe attachment is demonstrated by comparing Fig. 4(d) and 4(e). A negative control surface without a maleimide functional group was incubated with a PNA-Cy5 conjugate, and following a washing step some fluorescence is detected due to non-specific PNA adsorption. The silicon dioxide layer thickness is approximately $340 \mathrm{~nm}$ and quenching of the Cy5 fluorophore by the silicon is not possible in this case. The non-specific adsorption of PNA to glass is well known and we will return to this later in the article. A positive control surface containing an amine functional group following cross-linking activation and incubation with the PNA-Cy5 conjugate shows a uniform color change and confirmation of the PNA attachment to the gate-oxide surface. Subsequent studies of DNA-PNA hybridized duplexes attached to an oxide surface using radioactive labeling indicate surface densities $N_{\mathrm{d}} \sim 10^{12}$ molecules $\mathrm{cm}^{-2}$. 


\subsubsection{Electrical measurements}

Si-NW FET sensors measure surface charge density $\sigma_{0}$ on the gate-oxide sensor surface, from potential determining surface ions and/or the intrinsic electronic charge of hybridized biomolecular complexes attached to the sensor surface. The surface charge density induces a potential at the gate-oxide surface $\psi_{0} \approx 2 k T q^{-}$ ${ }^{1} \sinh ^{-1}\left(\sigma_{\mathrm{o}} / \beta\right)$, where $\beta=\left(8 \varepsilon_{\mathrm{w}} \varepsilon_{\mathrm{o}} k T c_{\mathrm{o}}\right)^{1 / 2}, \varepsilon_{\mathrm{w}}$ is the dielectric constant of the aqueous sample, $\varepsilon_{0}$ is the permittivity of free space and $c_{0}$ is the ionic strength of the background electrolyte ${ }^{17}$. In practice, Si-NW FET sensors measure a change in surface potential $\Delta \psi_{\mathrm{o}}$ from a quiescent state due to chemical interactions at the gate-oxide surface in the form of a sensor conductance change $\Delta G$ (i.e. $\Delta G=\Delta\left|i_{\mathrm{ds}}\right| v_{\mathrm{ds}}$, where $\Delta\left|i_{\mathrm{ds}}\right|$ is the change in the NW current and $v_{\mathrm{ds}}$ is the ac voltage applied across the length of the NW, as shown in Fig. 5(a)) in the silicon layer due to the electric field generated across the gate-oxide. Fig. 5(a) shows a sensor length cross-section and associated measurement circuit schematic. The back-gate bias voltage $\left(V_{b g}\right)$ controls the depletion behavior of the bottom side of the NW body, and the front-gate bias voltage $\left(V_{f g}\right)$ controls the depletion behavior of the top sensor surfaces that are in contact with the solution using the reference electrode. For all measurements presented in this article, the device operation was controlled with de voltage

(a)

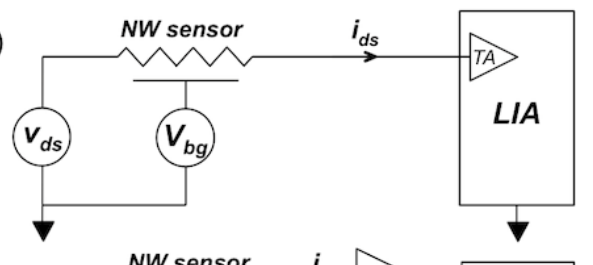

(b)

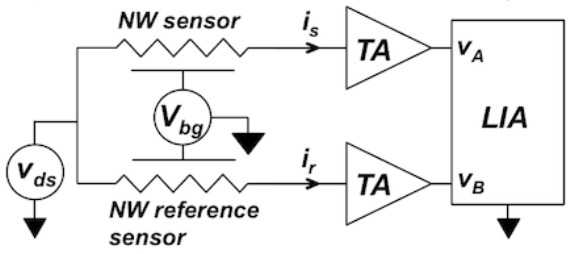

Figure 5. Electrical measurement configurations. (a) Single-device measurement circuit schematic. (b) Differential measurement circuit schematic.

sources $V_{f g}$ and $V_{b g}$ (2400 SMU, Keithley). The platinum wire reference electrode provides the front-gate bias in solution. The Si-NW is driven with an ac $(30 \mathrm{~Hz})$ modulation voltage $v_{\mathrm{ds}}$ and the ac device current is converted to a 
voltage with a transconductance amplifier (TA) and the magnitude and phase of the signal are measured with a lock-in amplifier (LIA) (SR 830, Stanford Research Systems). For single sensor measurements the internal TA of the LIA is used (Fig. 5(b)). The LIA uses quadrature synchronous demodulation, with an internal oscillator that is frequency matched to $v_{\mathrm{ds}}$, to retrieve the magnitude and phase of $i_{\mathrm{ds}}$. We report the root mean square magnitude of the measured signal from the LIA, i.e. $\left|i_{\mathrm{ds}}\right|$. All biasing and measurement instruments are controlled using a custom-made Labview program.

\subsubsection{1 $\mathrm{pH}$ and DNA hybridization sensor}

Following cleaning of the gate-oxide sensor surface, and subsequent surface modification for biosensing applications, the integrated microfluidic sensor cartridge is placed in the integrated system. PEEK tubing attached to the inlet port was connected directly to the pressurized reservoirs $\left(S R_{1}, S R_{2}\right.$ and $\left.S R_{3}\right)$, which are filled with $2 \mathrm{ml}$ of sample buffers with varying $p H$ or DNA concentrations. The buffer solution was pumped through the system. For $p H$ sensor experiments, the front-gate response curve $V_{f g}-\left|i_{d s}\right|$ was first recorded and analyzed in the initial $p H$ solution $(p H 4)$. All measurements were performed at ambient temperature, i.e. $20^{\circ} \mathrm{C}$. A pH gel electrode (PHC10101 electrode, Hach Lange) and multimeter (HQ40D multi, Hach Lange) were used for $p H$ measurements and calibration. The $\mathrm{pH}$-meter was calibrated at $\mathrm{pH} 4.0$ and $\mathrm{pH}$ 10.0 before each measurement. The NW current $\left|i_{\mathrm{ds}}\right|$ was recorded for $p H 7$ and 10 solutions, injected sequentially from $S R_{2}$ and $S R_{3}$, flowing through the microfluidic flow-cell. The measured $p H$ sensitivity $\Delta \psi_{\mathrm{o}} / \Delta p H$ was then calculated using the measured front-gate calibration curve. The hybridization buffer for DNA hybridization experiments is transported from $S R_{1}$ until the surface was stabilized, i.e. about 20 minutes. The different DNA concentration samples were then injected and the corresponding $\left|i_{\mathrm{ds}}\right|$ recorded for the duration of the experiment.

\subsubsection{Differential measurements}

A differential measurement configuration (Fig. 5(c)) provides the capability to cancel environmental sources of noise and interference, thus yielding measurements that better represent hybridization events compared to single sensor configurations. For differential measurements, two external TAs (SIM918, Stanford Research Systems) are used to convert the currents from the 
reference and sensor devices into voltages $v_{\mathrm{A}}$ and $v_{\mathrm{B}}$, respectively. The LIA measurement is then performed in differential mode $\left(v_{\mathrm{A}}-v_{\mathrm{B}}\right)$.

\subsubsection{System integration}

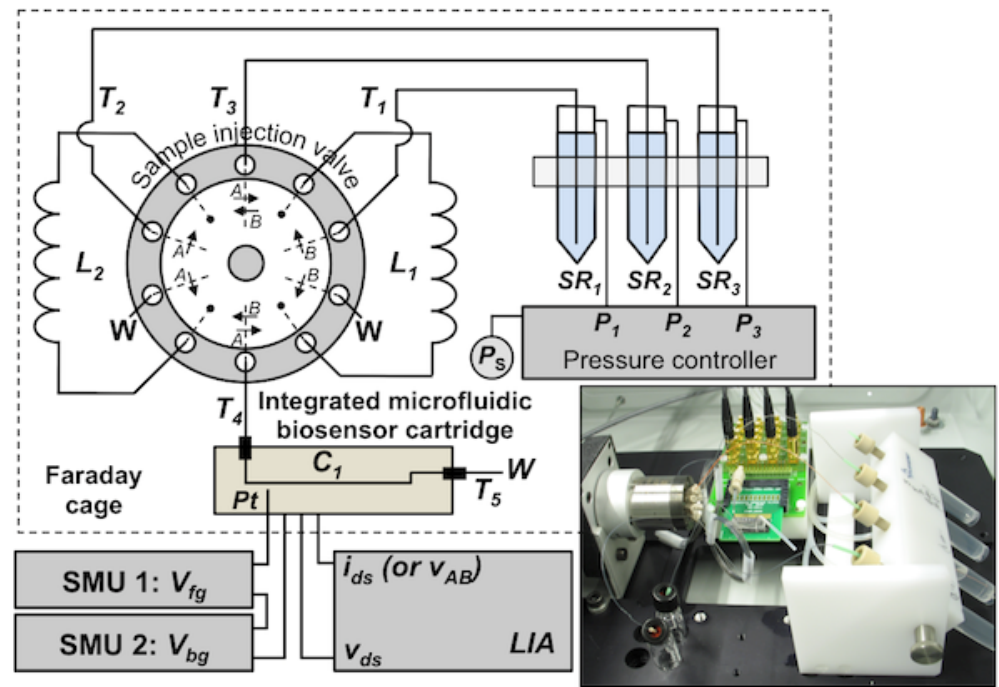

Figure 6. Schematic diagram of the integrated label-free analysis system with automated multi-sample delivery to the microfluidic chip. PEEK tubing is used for all hydraulic connections $T_{1}-T_{5}$ and sample loops $L_{1}$ and $L_{2} . C_{1}$ is the microfluidic flow-cell channel. The pressures $P_{1}-P_{3}$ control the flow rate in tubes $T_{1}-T_{3}$, respectively. The dotted enclosure represents the Faraday cage. The electrical biasing and measurement equipment are connected to the Si-NW chip using coaxial cables and a common ground point on the custom-made circuit board. Inset: Image of analysis system. The sample injection valve has two switch positions A and B.

The integrated (bio)chemical analysis platform consists of an analysis cartridge based on a wire bonded Si-NW biosensor array chip, functionalized with PNA ( in case of hybridization experiments), integrated with a microfluidic flow-cell, inserted into the automated multi-sample injection platform (Cheminert Nanovolume 10-port valve, Valco Instruments Co. Inc.) with PEEK fluidic connections for precise sample dosing, a regulated pressure source (MFCS-8C, Fluigent) for simultaneous sample transport, and electrical instrumentation for sensor readout as shown in Fig. 6. The electrical biasing and measurement equipment are connected to the Si-NW biosensors, denoted by SMU 1, SMU 2 and LIA. The microfluidic analysis cartridge and samples are housed in a Faraday cage to eliminate external electrical and ambient light noise sources. 
The sample injection valve is used to selectively deliver different sample solutions from sample reservoirs $S R_{1}, S R_{2}$ and $S R_{3}$, to the microfluidic flow-cell from pre-loaded sample loops, $L_{1}$ or $L_{2}$.

\subsection{Results and Discussion}

\subsubsection{Integrated platform}

Fig. 7 shows an equivalent hydraulic circuit diagram of the hydraulic system, where $R_{3}$ is the resistance of tube $T_{3}, R_{\mathrm{L}}$ is the resistance of the loop $L_{1}$ and $L_{2}$, $R_{4}$ is the resistance of tube $T_{4}$ and $R_{\mathrm{c}}$ is the resistance of the microfluidic flowcell microchannel. The volumetric flow rate is estimated with $Q \approx P_{2} / R_{\text {hyd }}$, where $R_{\text {hyd }}=R_{3}+R_{\mathrm{L}}+R_{4}+R_{\mathrm{c}}+R_{5}$. The average flow speed in the circular tube is estimated with $v_{\mathrm{s}} \approx Q / \pi a^{2}$. Table 2 shows flow speed estimations for different applied pressures.

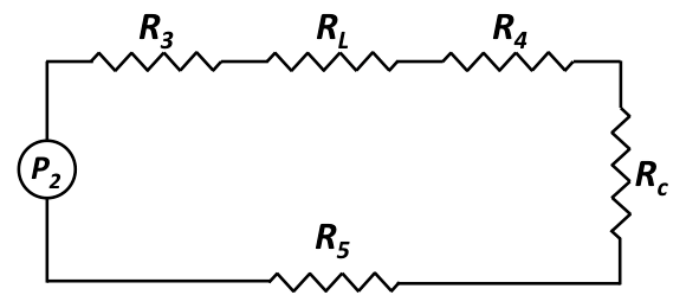

Figure 7. Equivalent hydraulic circuit diagram.

Table 2. Flow rates and flow speeds.

\begin{tabular}{|c|c|c|}
\hline $\begin{array}{c}\boldsymbol{P}_{\mathbf{2}} \\
\text { Bar }\end{array}$ & $\begin{array}{c}\boldsymbol{Q} \\
\mu 1 \mathrm{~min}^{-1}\end{array}$ & $\begin{array}{c}\boldsymbol{v}_{\mathbf{s}} \\
\mathrm{mm} \mathrm{s}^{-1}\end{array}$ \\
\hline 1.0 & 3.75 & 0.61 \\
\hline 0.5 & 1.88 & 0.31 \\
\hline 0.3 & 1.16 & 0.19 \\
\hline
\end{tabular}

For sensing experiments, the flow network has been designed to provide a sample flow velocity range of $0.1 \mathrm{~mm} \mathrm{~s}^{-1} \leq v_{\mathrm{s}} \leq 1 \mathrm{~mm} \mathrm{~s}^{-1}$ over an applied pressure range of 0.1 bar $\leq P_{\mathrm{i}} \leq 1$ bar and variable experimental duration. For equilibrium association and dissociation constant estimation of hybridized biomolecular complexes, a flow speed $v_{\mathrm{s}} \sim 1 \mathrm{~mm} \mathrm{~s}^{-1}$ has been used, which is compatible with the reaction-limited transport of target molecules to the sensor surface. 


\subsubsection{Silicon nanowire sensor sample flowrate dependence}

Since the Si-NW sensors are sensitive to surface potential changes, thus flow rate changes during sample injections can lead to significant and erroneous sensor responses due to the changes in the streaming potential at the gate-oxide surface induced by flowing electrolyte solutions ${ }^{18}$. Low ionic strength samples are most problematic, e.g. we have found that the electrical response of the SiNW sensors to varying flow rates of DI water and a low ionic strength buffer solutions, e.g. $1 \mathrm{mM} \mathrm{NaCl}$ buffer solution ( $p H 7.4$ ), can be significant and often interfere with biomolecular hybridization sensor responses, as shown in Fig. 8. The silicon nanowire (Si-NW) sensors are known to be sensitive to flow rate changes due to streaming potential changes on the sensor surface. ${ }^{18}$ Fig. 8 shows examples for $\mathrm{Si}-\mathrm{NW}$ sensor responses to changes in the flow rate in the integrated microfluidic flow-cell. Fig. 8(a) shows that the measured current can change by about $80 \%$ when the driving pressure is switched from $P_{1}=1$ bar to $P_{2}=0$ bar using a deionized water sample. Fig. 8 (b) shows about $14 \%$ change in current response
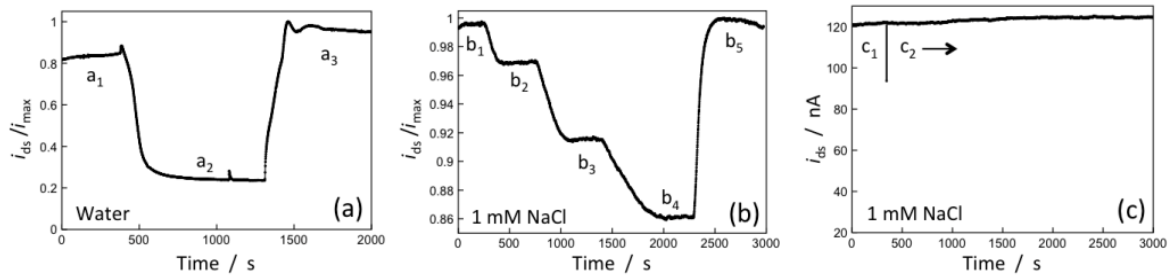

Figure 8. Measured Si-NW sensor current to flow rate changes in a microfluidic flow-cell. (a) Deionized water flow rate change as driving pressure is changed from $\mathrm{a}_{1}\left(P_{1}=1\right.$ bar) to $\mathrm{a}_{2}(0$ bar $)$ and then $\mathrm{a}_{3}\left(P_{1}=1\right.$ bar $)$. (b) Flow rate of $1 \mathrm{mM} \mathrm{NaCl}$ buffer as driving pressure is changed from $\mathrm{b}_{1}\left(P_{1}=1\right.$ bar $)$ to $\mathrm{b}_{2}\left(P_{1}=0.5\right.$ bar $)$ to $\mathrm{b}_{3}\left(P_{1}=0.2\right.$ bar) to $\mathrm{b}_{4}\left(P_{1}=0.1\right.$ bar $)$ and $\mathrm{b}_{5}\left(P_{1}=1\right.$ bar $)$. (c) Injector switch of $1 \mathrm{mM} \mathrm{NaCl}$ buffer from $c_{1}\left(S R_{1}, P_{1}=0.2\right.$ bar $)$ to $c_{2}\left(S R_{2}, P_{2}=0.2\right.$ bar $)$.

as the driving pressure is switched from $P_{1}=1$ bar to $P_{1}=0.1$ bar in a buffer solution containing $1 \mathrm{mM} \mathrm{NaCl}$. These results are consistent with previous reports, i.e. decreases in the flow rate result in a decrease in conductance of $\mathrm{p}$ type Si-NW devices and increased ionic strength reduces the effect of conductance changes from sample flow rate changes. ${ }^{18}$ Fig. 8(c) shows that flow rate changes due to sample switching can be eliminated with the automated multi-sample injection system and pressure driven flow. The Nanovolume sample injection valve, which has a small internal dead volume, and pressure 
driven sample transport provides stable and rapid sample switching when combined with a flow network that has balanced hydraulic resistances and provides stable baseline for further biosensing experiments.

\subsubsection{Si-NW operation and characterization}

Si-NW FET sensors measure surface potential changes $\Delta \psi_{0}$ on the sensor surface that originate from changes in the surface charge density $\Delta \sigma_{\mathrm{o}}$ due to changes in surface ions and/or the intrinsic charge of attached biomolecular complexes, as previously described. The surface potential change $\Delta \psi_{o}$ is determined from measurements of $\Delta G$ from homogeneously doped p-type depletion-mode Si-NWs. We recently reported that the conductance of depletion-mode Si-NWs can be operated in either the depletion or accumulation regions, thus the conductance is $G=\mathrm{q} \mu_{\mathrm{b}} N_{\mathrm{a}} L^{-1} \xi+2 \eta \mu_{\mathrm{a}} W_{\mathrm{a}} L^{-1} Q_{\mathrm{a}}$, where $q$ is the electronic charge, $\mu_{\mathrm{b}}$ is the dopant concentration dependent bulk hole mobility, $N_{\mathrm{a}}$ is the boron impurity dopant concentration in the NW body, $L$ is the device length, $\xi$ is a gating function that is dependent on $\psi_{o}, \mu_{\mathrm{a}}$ is the field dependent hole mobility in the accumulated layer, $W_{\mathrm{a}}$ is the total surface area of the accumulated surface and $Q_{\mathrm{a}}$ is the electronic charge in the accumulation layer. ${ }^{19}$ For all measurements presented in this article, the Si-NWs are operated in the depletion region where $Q_{a}=0$ since $\left|V_{\mathrm{fg}}\right|<\left|V_{\mathrm{fb}}\right|$, where $V_{\mathrm{fb}}$ is the flatband voltage that represents all potential drops across the gate-oxide/silicon interface, which will be described later. It should be noted that the conductance associated with the metal/semiconductor contact region has been carefully designed and characterized to have a negligible contribution to the total device conductance, and therefore, has been neglected in the conductance model derivation.

The conductance change for Si-NWs biased in the depletion region can be approximated with $\Delta G \approx q \mu_{\mathrm{b}} N_{\mathrm{a}} L^{-1} \Delta \xi$, where $\Delta \xi \approx\left(W-(2)^{1 / 2} \Delta f_{\mathrm{d}}\right)\left(a-(2 / 3)^{1 / 2} \Delta f_{\mathrm{d}}\right)$ is the area of the conductance cross-section of the triangular Si-NW with width W and height $\mathrm{a}$, which is modulated by a depletion length function $\Delta f_{\mathrm{d}} \approx\left(\gamma^{2} t_{\mathrm{ox}}{ }^{2}+2 \varepsilon_{\mathrm{Si}} \varepsilon_{\mathrm{o}}\left(V_{\mathrm{fg}}-\Delta V_{\mathrm{fb}}\right) / q N_{\mathrm{a}}\right)^{1 / 2}-\gamma^{2}{t_{\mathrm{ox}}}^{2}$ with $\gamma=\varepsilon_{\mathrm{Si}} \varepsilon_{\mathrm{OX}}{ }^{-1}$, where $\varepsilon_{\mathrm{Si}}$ and $\varepsilon_{\mathrm{OX}}$ are the permittivities of silicon and silicon dioxide, respectively, $t_{o x}$ is the gate-oxide thickness, and finally the flatband voltage change is proportional to the surface potential change $\Delta V_{f b}=\zeta-\Delta \psi_{o}$ with $\zeta=E_{\mathrm{ref}}-q^{-1} \phi_{\mathrm{Si}}-Q_{\mathrm{f}} C_{\mathrm{o}}{ }^{-1}-Q_{\mathrm{ss}} C_{\mathrm{o}}{ }^{-1}+\chi^{\text {sol }}$ where $E_{r e f}$ is the reference electrode potential, $q^{-1} \phi_{\mathrm{Si}}$ is the work function of silicon, $Q_{f}$ and $Q_{s s}$ are the fixed charge and interface states near the gate-oxide/silicon interfaces, 
respectively, $C_{\mathrm{o}}=\varepsilon_{\mathrm{Si}} \varepsilon_{0} t_{\mathrm{ox}}{ }^{-1}$ is the gate-oxide capacitance and $\chi^{\text {sol }}$ is the surface dipole potential of the sample solution. The flatband voltage provides a direct physical link between $\Delta \psi_{o}$ and $\Delta G$. It has been assumed that the buriedoxide/silicon interface is not depleted and the back-gate is not electrostatically coupled to the front-gate, which is accomplished in practice by choosing an appropriate $V_{b g}$. This brief overview of the basic physical principles of the FET Si-NW sensor operation highlight a few important points that are useful to summarize: $i$. the application of a dual gate biasing configuration with a reference electrode in the sample buffer ensures optimal and reproducible device behavior; ii. the sensor surface should be well cleaned (e.g. short duration UV ozone) and the detrimental effects from $Q_{f}$ and $Q_{s s}$ at the gate-oxide/silicon interface minimized through thermal annealing; and iii. The contact resistance of the drain and source contacts should be minimized such that it represents a small fraction of the total quiescent sensor resistance.

The Si-NWs were characterized prior to experiments to assess the frontgate $V_{f g}-\left|i_{d s}\right|$ and $g_{m^{-}}-i_{d s} \mid$ response curves, where $g_{m}=\partial i_{d s} \mid / \partial V_{f g}$ (at fixed $v_{d s}$ ) is the sensor transconductance. Front-gate biasing affects the depletion region differently than back-gate biasing, as previously described, and we expect higher surface charge sensitivity for biosensor applications with this configuration. ${ }^{20}$ For biosensor applications, the devices can be biased in the region with highest transconductance and linear $\left|i_{d s}\right|$ range while maintaining a low front-gate current $i_{f g}<1 \mathrm{nA} .{ }^{19}$ The back-gate bias $V_{b g}$ is determined and fixed during the initial calibration. Fig. 9(a) shows a representative example of an $V_{f g}-|i d s|$ response curve of a Si-NW where the front-gate voltage is scanned with a platinum reference electrode in a low ionic strength buffer $(p H 4)$ and $\left|i_{d s}\right|$ recorded. The operation region transitions from depletion to the accumulation as $V_{f g}$ is scanned to larger negative voltages. The decrease in transconductance for $V_{f g}$ beyond the maximum is due to a decrease in field-dependent effective mobility $\mu_{a}$ and charge screening. For all experiments, the Si-NW devices are biased with $V_{f g}=-0.6 \mathrm{~V}$ and $V_{b g}=-7.5 \mathrm{~V}$, unless otherwise specified.

\subsection{4. pH sensor}

Si-NW $p H$ sensors measure the potential difference $\psi_{o}$ across the electrical double layer at the interface between an electrolyte solution and the oxide sensor surface, which is related to the activity of the potential determining ions and 
described by the Nernst equation $\psi_{\mathrm{o}}=2.303 k T q^{-1} \log \left[a_{\mathrm{H}^{+}} /{ }^{\circ} a_{\mathrm{H}^{+}}\right]$, where $a_{\mathrm{H}^{+}}$is the ion activity of the solution, and ${ }^{\mathrm{o}} a_{\mathrm{H}^{+}}$is the ion activity at the point of zero charge. Therefore, the maximum variation $\psi_{o}$ can take is $58 \mathrm{mV}$ per decade of ion activity at the standard temperature of $293 \mathrm{~K}\left(20^{\circ} \mathrm{C}\right)$. For an oxide surface, the $\mathrm{H}^{+}$ion is potential determining, which is assigned to the oxide surface, and counter-ions present in solution are from the background electrolyte and can be neglected at low ionic concentrations. The solution $\mathrm{pH}$ is related to the $\mathrm{H}^{+}$ion activity, and therefore, the experimental measurement of $\Delta \psi_{o}$ is obtained through titration of the oxide in the presence of a supporting electrolyte by changes in $\mathrm{pH}$. The corresponding maximum $\mathrm{pH}$ sensitivity of a surface is, therefore, limited to $\Delta \psi_{o} / \Delta p H=2.303 \mathrm{kTq}^{-1}$.

The $\mathrm{pH}$ measurements using the integrated microfluidic Si-NW sensor platform were done using $v_{d s}=100 \mathrm{mV}, V_{b g}=-7.5 \mathrm{~V}$ and UV ozone cleaned gateoxide sensor surfaces. First, a $p H 4$ buffer solution was transported through the microfluidic flow-cell for 20 minutes to establish a stable baseline signal, and followed by the injection of a buffer solution with $\mathrm{pH} 7$ for 2 minutes and finally the injection of a $p H 10$ buffer for $3 \mathrm{~min}$, all at a flow rate of $0.5 \mu \mathrm{L}$ min-1, which corresponds to an average flow speed $v_{\mathrm{s}} \approx 0.1 \mathrm{~mm} \mathrm{~s}^{-1}$. For p-type depletion mode $\mathrm{Si}-\mathrm{NWs}$, increasing the solution $\mathrm{pH}$ results in an increasingly negative surface potential change, which results in a reduced depletion region of the depletion-mode Si-NW devices, and therefore, an increase in $\left|i_{d s}\right|$. Fig. 9(b) shows the $p H$ response of the integrated Si-NW sensor system to different $\mathrm{pH}$ sample solutions. From Fig. 9(a), the slope of the $V_{f g}-\left|i_{d s}\right|$ response curve is $\Delta\left|i_{\mathrm{ds}}\right| / \Delta V_{\mathrm{fg}} \approx-2.6 \mathrm{mV} / \mathrm{nA}$ (at $V_{\mathrm{fg}}=-0.6 \mathrm{~V}$ ) over the $p H$ experiment range. The rightside ordinate of Fig. 9(b) shows the estimated surface potential change as the solution is increased from $p H 4$ to $p H 10$. Note, the surface potential change is estimated from the initial experimental sample with $p H 4$. The bare gate-oxide surfaces demonstrate the well-known nonlinearity for low $p H$ values near the point of zero charge $\left(\mathrm{pH}_{\mathrm{pzc}} \approx 2\right)$, which becomes linear for $\mathrm{pH}$ values above about 5. ${ }^{16,19}$ The measured current shows a sharp increase followed by a gradual increase to the steady state response for an increase in $\mathrm{pH}$ from 7 to 10 , which is characteristic of the time dependent surface equilibration of the oxide surface. Over the range $7 \leq p H \leq 10$ the $\mathrm{pH}$ sensitivity is $\Delta \psi_{\mathrm{o}} / \Delta p H \approx 48 \mathrm{mV} / \mathrm{pH}$, which is consistent with measurements in previous experimental results using conventional laboratory sample volumes. ${ }^{19}$ This measurement demonstrates that the integrated system can provide stable and well-controlled $p H$ sensing with 
small sample volumes over the time scale of minutes. Other sensing surfaces, such as $\mathrm{Al}_{2} \mathrm{O}_{3}$ or $\mathrm{Ta}_{2} \mathrm{O}_{5}$, which are well known to provide near Nernstian sensitivity and linear $p H$ responses, ${ }^{19,}{ }^{21}$ can be easily incorporated into the SiNW sensing chip.

\subsubsection{DNA hybridization}

For DNA measurements on Si-NWs the ionic strength of the buffer is important to determine the Debye length of the solution. The Debye length is defined as $\lambda_{\mathrm{D}}=\left(\varepsilon_{\mathrm{w}} \varepsilon_{0} k T / 2 z^{2} q^{2} c_{\mathrm{o}}\right)^{1 / 2}$, where $z$ is the valence ionic charge and co is the ionic concentration. For example, $\lambda_{\mathrm{D}} \approx 1 \mathrm{~nm}$ for $c_{o}=100 \mathrm{mM}$ and $\lambda_{\mathrm{D}} \approx 10 \mathrm{~nm}$ for $c_{o}=1$ $\mathrm{mM}$ for a monovalent ion electrolyte solution. Binding of negatively charged DNA biomolecules near the Si-NW gate-oxide surface induces a change in the surface potential $\Delta \psi_{0}$, which results in $\Delta G$ of the Si-NW, as previously discussed, provided that the intrinsic biomolecular charge is not screened due to ionic species in the electrical double layer at the gate-oxide surface; ${ }^{22}$ i.e. the Debye length $\left(\lambda_{\mathrm{D}}\right)$ of the buffer solution at the gate-oxide surface should not be too small (i.e. $\lambda_{\mathrm{D}}>5 \mathrm{~nm}$ for vertically attached probes). Counter-ion screening, or counter-ion condensation, has also been reported for DNA molecules where the negative phosphate backbone charges are screened by cations in the electrolyte, thus reducing the intrinsic DNA charge by up to $80 \%$ for high ionic strength solutions. ${ }^{23}$ Therefore, low ionic strength $(<100 \mathrm{mM})$ hybridization buffers are preferred for the highest sensitivity detection. However, low ionic strength hybridization buffers are not suitable for high efficiency DNA-DNA duplex formation. For this purpose we use synthetic PNA probes, mimics of oligonucleotides with a charge neutral peptide backbone, which are reported to have low equilibrium dissociation constants to complementary DNA in low ionic strength buffers that have a relatively large Debye length; the DNA-PNA duplex formation is less dependent on the ionic strength of the hybridization buffer compared to DNA-DNA duplex formation. ${ }^{24-26}$ We present real-time DNA-PNA duplex hybridization measurements of complementary (M0) DNA target molecules to PNA probe molecules covalently conjugated to the silicon oxide gate-oxide surface of 2-wire Si-NW sensors (Fig. 2(b)) in a low ionic strength buffer. The measurements have been performed with sample flow speeds high enough to ensure reaction-limited transport of the target DNA to the sensor surface and we use the first-order Langmuir binding model to assess the 
equilibrium dissociation constant $K_{D}$ of the DNA-PNA duplex hybridization process. The equilibrium dissociation constant is defined as $K_{D}=k_{a} / k_{d}$, where $k_{a}$ is the association rate constant that represents the speed of the second-order probe-target interaction and $k_{d}$ is the dissociation rate constant that represents the speed of the first-order breakdown of the probe-target complex. Thermodynamics dictates the target-probe hybridization process and a small $K_{D}$ value indicates a high affinity of the probe for the target, which generally lies in the $10^{-15} \mathrm{M}<K_{D}<10^{-6} \mathrm{M}$ range. ${ }^{27}$ From mass action kinetics, the time dependent surface density formation of a single type of probe-target complex on the surface can be monitored in real-time with the response function $R(t)=R_{0}(1-\exp [-$ $\left.\left.\left(k_{\mathrm{a}}[C]+k_{\mathrm{d}}\right) t\right]\right)$, where $R_{\mathrm{o}}=\left(k_{\mathrm{a}} R_{\max }[C]\right) /\left(k_{\mathrm{a}}[C]+k_{\mathrm{d}}\right), R_{\text {max }}$ is the maximum sensor signal response for a given probe density $N_{p}$ and $[C]_{i}$ is the concentration of the target analyte. The time dependent response function, which is derived by solving a rate equation that includes the association and dissociation of target molecules with surface probe molecules as a function of time, is commonly referred to as the Langmuir equation. The real-time response measured from the $\mathrm{Si}-\mathrm{NW}$ sensors is drain-source current, thus $R(t) \equiv\left|i_{d s}\right|(t)$ for fixed electrical biases $V_{f g}, V_{b g}$ and $v_{d s}$, and target concentration $[C]_{\mathrm{i}}$ for each measurement. Fig. 9(c) shows measured real-time current responses for injections of M0 DNA with $[C]_{2, M 0}=100 \mathrm{nM}$ and $[C]_{3, M 0}=500 \mathrm{nM}$ concentrations in $1 \mathrm{mM}$ phosphate buffer $\left(\lambda_{\mathrm{D}} \approx 10 \mathrm{~nm}\right)$ to complementary PNA probe molecules conjugated to the gateoxide surface with a sample flow speed of $v_{s} \approx 0.6 \mathrm{~mm} \mathrm{~s}^{-1}$. The sensor reponse to the injection of NC DNA with $[C]_{1, N C}=100 \mathrm{nM}$ is also shown in Fig. 9(c), which is the baseline noise of the measurement. The $500 \mathrm{nM}$ concentration M0 DNA produces $\Delta\left|i_{d s}\right| \approx 1 \mathrm{nA}$ and $100 \mathrm{nM} \mathrm{M0}$ DNA produces $\Delta\left|i_{d s}\right| \approx 0.4 \mathrm{nA}$, as shown in Fig. 9(c). The estimated limit of detection is $50 \mathrm{nM}$, using a 3-sigma cutoff of the measured NC $100 \mathrm{nM}$ data noise, for the experimental conditions used in this article and can be reduced, which will be discussed later in this section. The increase in conductance with an increase of negatively charged molecules on the gate-oxide sensor surface of the p-type depletion mode sensor is expected from the sensor characterization (Fig. 6(a)). We have measured the density of DNAPNA duplexes formed on oxide surfaces in equilibrium, using a $\mathrm{P}^{32}$ radioactive assay (data not shown), and found that a saturated surface contains about $\mathrm{N}_{\mathrm{d}} \sim 10^{12}$ duplexes $\mathrm{cm}^{-2}$, which corresponds to a lower limit $\Delta \psi_{o} \sim 10 \mathrm{mV}$ using an oxide charge density $\sigma_{o x} \approx 0.2 \mathrm{C} \mathrm{m}^{-2}$ for a buffer with $\mathrm{pH} 7 .^{19}$ The estimated 
surface potential $\Delta \psi_{o} \approx\left(2 k T q^{-1}\right)\left(\sinh ^{-1}\left(\left(\sigma_{D N A^{-}} \sigma_{o x}\right) / \beta\right)-\sinh ^{-1}\left(\sigma_{o x} / \beta\right)\right)$, where $\varepsilon_{w}=80$ and $c_{o}=1 \mathrm{mM}$. The charge density of a saturated 15-mer DNA-PNA surface is estimated with $\sigma_{D N A} \approx \mathrm{N}_{\mathrm{d}} \times 15 \times \mathrm{q} \times 10^{4} \mathrm{C} \mathrm{m}^{-2}$.

The estimated surface potential changes correspond to larger conductance changes compared to the measured conductance changes and we are currently working to optimize the PNA probe sensor surface. The association and dissociation rate constants, $k_{\mathrm{a}}$ and $k_{\mathrm{d}}$, are extracted by fitting the measured current responses to the kinetics reponse function using all of the experimental concentrations.
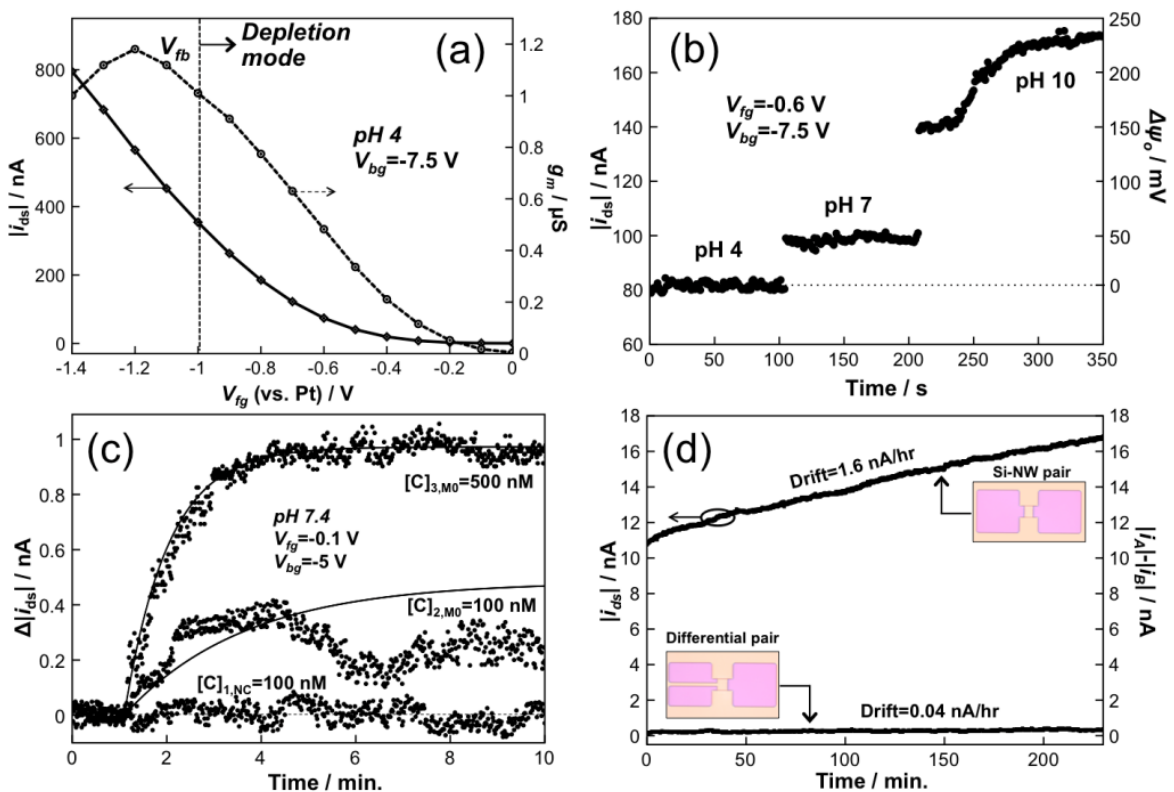

Figure 9. Integrated biosensor analysis platform measurements. (a) Si-NW frontgate biasing characteristics in $p H 4$ buffer solution with platinum reference electrode. (b) $p H$ measurements with flow speed $\mathrm{v}_{\mathrm{s}} \approx 0.1 \mathrm{~mm} \mathrm{~s}^{-1}$. (c) DNA-PNA duplex hybridization detection with $[C]_{3, M 0}=500 \mathrm{nM},[C]_{2, M 0}=100 \mathrm{nM}$ and $[C]_{1, N C}=100 \mathrm{nM}$ target concentrations and flow speed $v_{s} \approx 0.6 \mathrm{~mm} \mathrm{~s}^{-1}$. (d) Differential measurement in buffer solution.

The solid lines in Fig. 9(c) represent the model fit to the measured data with $R_{\max } \approx 1 \mathrm{nA}, k_{\mathrm{a}} \approx 3 \times 10^{4} \mathrm{M}^{-1} \mathrm{~s}^{-1}$ and $k_{\mathrm{d}} \approx 4 \times 10^{-3} \mathrm{~s}^{-1}$, which gives $K_{\mathrm{D}}=140 \mathrm{nM}$. The $R_{\max }$ value is consistent with $[C]_{3, M 0}>\mathrm{K}_{\mathrm{D}}$, where all PNA binding sites are assumed to be occupied. The extracted $K_{\mathrm{D}}$ is much larger than dissociation constants extracted from measurements of the DNA-PNA duplex hybridization. $^{25,26,28}$ A dissociation constant of $K_{\mathrm{D}} \approx 350 \mathrm{nM}$ for a DNA-PNA 
duplex hybridization at $35{ }^{\circ} \mathrm{C}$ has been reported. ${ }^{29}$ The discrepancy between the measurements can be due to differences in the PNA attachment scheme. ${ }^{26}$ Extracted $K_{\mathrm{D}}$ values of DNA-DNA duplex hybridization measured using Si-NW sensors in a $165 \mathrm{mM}$ ionic strength buffer are similar to our measurements. ${ }^{12}$

We have found in subsequent studies that PNA molecules nonspecifically adsorb to many types of surfaces. Although it is well known that PNA molecules non-specifically adsorb to glass surfaces (Eurogentec), they also non-specifically adsorb to silicon nitride $\left(\mathrm{Si}_{3} \mathrm{~N}_{4}\right)$, PDMS and polyimide surfaces with similar attachment densities that are difficult to remove in most cases (data not shown). The non-specific adsorption of PNA on the polyimide surface is problematic and can negatively affect the estimation of the dissociation rate $k_{\mathrm{d}}$ and the limit of detection to target depletion. Following the kinetics and transport analysis that describe the interaction of target analytes with probe molecules on surfaces, ${ }^{30-32}$ we can further assess the validity of our measurements. From the channel dimensions and sample flow rate, the transport of target DNA to the sensing surface is reaction-limited since the Damkohler number $D a \approx 2 \times 10^{-3}$, thus verifying the use of the single complex formation response function. From Fig. $9(\mathrm{c})$, the time constant $\tau_{\mathrm{r}}=\left(k_{\mathrm{a}}[C]_{\mathrm{i}}+k_{\mathrm{d}}\right)^{-1}$ of the 100 $\mathrm{nM}$ response $\tau_{\mathrm{r}} \approx 2$ minutes and the $500 \mathrm{nM}$ response has $\tau_{\mathrm{r}} \approx 1$ minute, which is much smaller than the time constants measured in previous DNA-PNA duplex formation studies where $\tau_{\mathrm{r}} \sim 10$ minutes have been reported. ${ }^{25,26}$ Fast DNA-PNA and DNA-DNA duplex hybridization times have been reported in other Si-NW sensor studies. ${ }^{12,33}$ More work is required to understand these differences and to optimize the PNA probe density and minimize the non-specific adsorption of PNA to surfaces that interface with the target DNA.

\subsubsection{Differential sensing}

Sensor drift is the systematic increase or decrease of the sensor response as a function of measurement time. It is well known that the output response of ion sensitive field-effect transistor sensors, the microscale predecessors to Si-NW FET sensors, suffer from drift due to ion migration at the gate-oxide interface. ${ }^{8}$, ${ }^{34}$ A typical response of the 2-wire Si-NW sensor (Fig. 2(b)) with a silicon dioxide surface, measured with the single device measurement configuration shown in (Fig. 2(d)), in a $1 \mathrm{mM} \mathrm{NaCl}$ buffer solution over a period of about 4 hours, is shown in the upper curve in Fig. 9(d). The drift rate of $1.6 \mathrm{nA} \mathrm{hr}^{-1}$ is 
significant considering the quiescent current $\left|i_{\mathrm{ds}}\right| \approx 10 \mathrm{nA}$. Note, the left ordinate scale represents the measured current from the single device measurement configuration. A differential measurement configuration can be used to improve the output response of the Si-NW sensors. Fig. 5(c) shows an example of the differential measurement configuration where the current is divided into reference and sensor currents, $i_{\mathrm{S}}$ and $i_{\mathrm{r}}$, respectively, using the split-source SiNW device (Fig. 2(d)). The currents are converted to voltages $v_{\mathrm{A}}$ and $v_{\mathrm{B}}$ with external TAs and the LIA output in differential measurement mode represents their difference, or null value $\left(v_{\mathrm{A}}-v_{\mathrm{B}}\right)$. A typical differential response is shown in the lower curve of Fig. 9(d), where the drift rate has been significantly reduced by a factor of $\sim 30 \times$ to $0.04 \mathrm{nA} \mathrm{hr}^{-1}$. Note, the right ordinate scale represents the difference of the measured currents from the differential measurement configuration. The quiescent output signal is ideally nulled to zero (in this case the offset current is $0.1 \mathrm{nA}$ due to device mismatch), and therefore, changes in the sensor signal $\Delta i_{\mathrm{s}}$ can be detected with a higher sensitivity as the detection range of the instrument can be reduced. If the sensor and reference devices are each replaced by $N$-parallel connected Si-NW devices then the detection response will be ideally amplified by a factor of $N \times$. For example, when the single sensor and reference devices are replaced by a parallel array of $N$-Si-NWs the ideal nulled output signal is $i_{\mathrm{s}}-i_{\mathrm{r}}=N \Delta i$, where $\Delta i$ is current change from a single NW sensor induced from a surface potential change $\Delta \psi_{0}$. The LIA measurement instrument readout is a voltage difference $v_{\mathrm{A}}-v_{\mathrm{B}} \approx-$ $N v_{\mathrm{ds}}\left(R_{\mathrm{f}} / R_{\mathrm{NW}}\right)\left(\Delta R / R_{\mathrm{NW}}\right)$, where $R_{\mathrm{f}}$ is the gain of the TA, $R_{\mathrm{NW}}$ is the quiescent resistance of the NW and $\Delta R$ is the change in the NW resistance induced $\Delta \psi_{0}$.

\subsection{Conclusions}

We have presented an integrated microfluidic label-free analytical platform that can be used for small volume chemical analysis, such as $p H$ or ion sensing, as well as diagnostics for the specific measurement of biomolecules and for the kinetics estimation of biomolecular hybridization. We have integrated a scalable silicon nanowire sensor chip with a microfluidic flow-cell into a simple disposable cartridge format that is mounted into the all-electrical readout instrument. An automated multi-sample injection system was presented that provides fast and reliable sample switching without spurious flow rate fluctuations that can produce erroneous sensor signals. Measurements of $p H$ 
from different samples have been presented with sensitivities consistent with a silicon dioxide sensor surface. Real-time DNA-PNA duplex hybridization measurements have been presented with different sample concentrations in a low ionic strength buffer, and the equilibrium dissociation constant $K_{\mathrm{D}} \approx 140 \mathrm{nM}$ has been extracted from the experimental data using the first order Langmuir binding model. Finally, a differential sensor configuration has been presented and a $30 \times$ reduction in sensor drift has been shown. The integrated microfluidic label-free analysis platform with measurement cartridge based on an electronic Si-NW biosensor array, integrated with a simple microfluidic flow-cell, has been demonstrated by measuring different sample types. The Si-NW (bio)chemical sensor cartridge has been developed for an automated molecular diagnostic system integrated in an ingestible pill, and is a promising platform for low cost (bio)chemical diagnostics and drug screening. 


\section{References}

1. P. Bertone and M. Snyder, FEBS Journal, 2005, 272, 5400-5411.

2. N. Ramachandran, D. N. Larson, P. R. H. Stark, E. Hainsworth and J. LaBaer, FEBS Journal, 2005, 272, 5412-5425.

3. W. Gu, Trends in Bio/Pharmaceutal Industry, 2009, 43.

4. J. Comley, Drug Discovery World, 2008, 9, 77-88.

5. J. Homola, S. S. Yee and G. Gauglitz, Sensors and Actuators, B: Chemical, 1999, 54, 3-15.

6. G. Krishnamoorthy, E. T. Carlen, J. G. Bomer, D. Wijnperlé, H. L. Deboer, A. van den Berg and R. B. M. Schasfoort, Lab Chip, 2010, 10, 986-990.

7. P. M. Levine, P. Gong, R. Levicky and K. L. Shepard, Biosensors and Bioelectronics, 2009, 24, 1995-2001.

8. P. Bergveld, Sensors and Actuators, 1981, 1, 17-29.

9. J. Fritz, E. B. Cooper, S. Gaudet, P. K. Sorger and S. R. Manalis, Proceedings of the National Academy of Sciences of the United States of America, 2002, 99, 14142-14146.

10. E. T. Carlen and A. van den Berg, Lab Chip, 2007, 7, 19-23.

11. Y. Cui, Q. Wei, H. Park and C. M. Lieber, Science, 2001, 293, 1289-1292.

12. Y. L. Bunimovich, Y. S. Shin, W. S. Yeo, M. Amori, G. Kwong and J. R. Heath, Journal of the American Chemical Society, 2006, 128, 16323-16331.

13. S. Chen, J. G. Bomer, W. G. Van der Wiel, E. T. Carlen and A. van den Berg, ACS Nano, 2009, 3, 3485-3492.

14. E. H. Snow, A. S. Grove and Fitzgera.Dj, Proceedings of the Institute of Electrical and Electronics Engineers, 1967, 55, 1168-1185

15. B. E. Deal, Journal of the Electrochemical Society, 1974, 121, C198-C205.

16. L. Bousse, N. F. De Rooij and P. Bergveld, IEEE Transactions on Electron Devices, 1983, ED-30, 1263-1270.

17. J. Israelachvili, Intermolecular and Surface Forces, Academic Press London, 1992.

18. D. R. Kim, C. H. Lee and X. Zheng, Nano Letters, 2009, 9, 1984-1988.

19. S. Chen, J. G. Bomer, E. T. Carlen and A. van den Berg, Nano Letters, 2011, 11, 2334-2341.

20. H. D. Tong, S. Chen, W. G. Van Der Wiel, E. T. Carlen and A. van den Berg, Nano Letters, 2009, 9, 1015-1022. 
21. O. Knopfmacher, A. Tarasov, W. Fu, M. Wipf, B. Niesen, M. Calame and C. Schönenberger, Nano Letters, 2010, 10, 2268-2274.

22. J. Janata, In Proc. of the 2nd Int. Meeting on Chemical Sensors. Ed. J.L. Aucouturier et al., Bordeaux, France, 1986, 25.

23. G. S. Manning, Quarterly Reviews of Biophysics, 1978, 11, 179-246.

24. M. Egholm, O. Buchardt, L. Christensen, C. Behrens, S. M. Freier, D. A. Driver, R. H. Berg, S. K. Kim, B. Norden and P. E. Nielsen, Nature, 1993, 365, 566-568.

25. J. Liu, L. Tiefenauer, S. Tian, P. E. Nielsen and W. Knoll, Analytical Chemistry, 2006, 78, 470-476.

26. H. Park, A. Germini, S. Sforza, R. Corradini, R. Marchelli and W. Knoll, Biointerphases, 2007, 2, 80-88.

27. D. A. L. a. J. J. Linderman, Receptors: Models for Binding, Trafficking, and Signaling, Oxford University Press, New York, 1993.

28. F. Yu, D. F. Yao and W. Knoll, Nucleic Acids Research, 2004, 32.

29. K. Kilså Jensen, H. Ørum, P. E. Nielsen and B. Nordén, Biochemistry, 1997, 36, 5072-5077.

30. S. Sjölander and C. Urbaniczky, Analytical Chemistry, 1991, 63, 2338-2345.

31. M. Zimmermann, E. Delamarche, M. Wolf and P. Hunziker, Biomedical Microdevices, 2005, 7, 99-110.

32. T. M. Squires, R. J. Messinger and S. R. Manalis, Nature Biotechnology, 2008, 26, 417-426.

33. J. I. Hahm and C. M. Lieber, Nano Letters, 2004, 4, 51-54.

34. R. G. Kelly, Electrochimica Acta, 1977, 22, 1-8. 
Chapter 5 


\section{Chapter 6}

\section{DNA probe layer surface chemistry ${ }^{4}$}

The biomolecular interface, between a target molecule in the sample solution and a sensor surface, is a key component to ensure both high detection specificity and sensitivity. The detection specificity depends on how well the target binds with the receptor (probe) molecule, which is tethered to the sensor surface, as well as the probe surface density [molecules $\mathrm{cm}^{-2}$ ], such that the steric hindrance does not inhibit the hybridization process. In this chapter, the methods, techniques, and characterization results of the surface probe interfaces are described, which was developed for the electronic detection of deoxyribonucleic acid (DNA) using silicon nanowire (Si-NW) biosensors that are integrated into a small volume microfluidic flow chamber.

${ }^{4}$ Adapted in part from: A. De, J. van Nieuwkasteele, E.T. Carlen and A. van den Berg, Integrated label-free silicon nanowire sensor arrays for (bio)chemical analysis. Analyst, 138, 3221, 2013; and A. De, S. Souchelnytyski, A. van den Berg and E.T. Carlen, Peptide nucleic acid (PNA)-DNA duplexes: Comparison of hybridization affinity between vertically and horizontally tethered PNA probes, ACS Appl. Mater. Interfaces, 2013, 5, 4607-4612. 


\subsection{Introduction}

A conventional biomolecular surface probe layer consists of a covalently attached probe moiety tethered at one end to a solid surface via a linker molecule, as shown in the illustration of different probe-target complexes in Fig. 1. The probe molecule can be anything from DNA, ribonucleic acid (RNA), peptide nucleic acid (PNA), antibodies, and proteins that hybridize to their respective complementary target. The linker layer with functional chemical groups at both ends, such as amine $\left(\mathrm{NH}_{2}\right)$, aldehyde $(\mathrm{COH})$ and carboxyl $(\mathrm{COOH})$ groups, which covalently attaches the probe to the solid surface in one or more chemical processing steps.

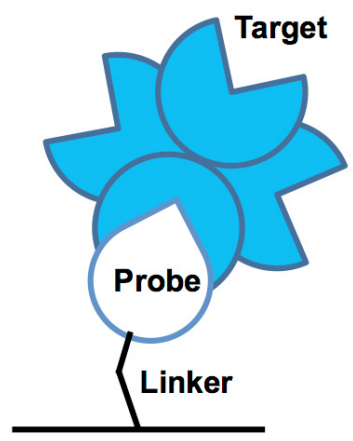

(a)

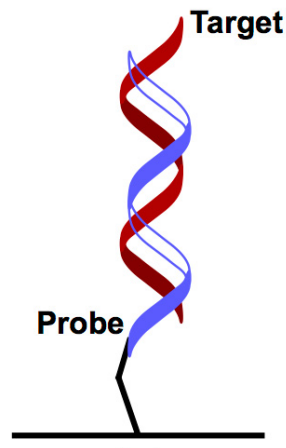

(b)

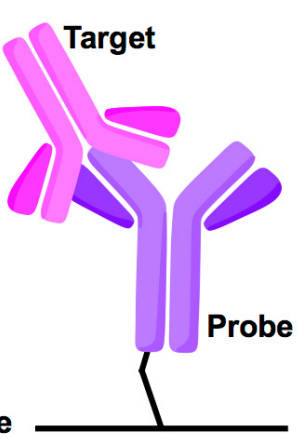

(c)

Figure 1. Schematics for different types of biomolecular probe-target complexes tethered to the solid surface. (a) biotin-streptavidin (b) PNA-cDNA and (c) antibody IgG-antiIgG

The probe layer is a critical component of the target detection system to ensure both high detection specificity and sensitivity. The specificity of detection resides on the level of target hybridization to the covalently bound probe on the sensor surface, and the probe surface density, such that steric hindrance does not restrict the hybridization process.

The hybridization of DNA on solid surfaces is a well-established and mature field due to the development of microarrays for genomics. In this work, we benefit from the vast knowledge that is described in the microarray literature of surface preparation schemes of glass surfaces. Glass has been the material of choice for microarray formation because it has a number of practical advantages over porous membranes and gel pads. ${ }^{1}$ For the covalent attachment to glass surfaces, aminated DNA is attached to the glass surface using a covalent bond 
between the primary amine and chemical moieties on the glass surface, as oligonucleotides (oligos) cannot be coupled directly to the silanol groups (Si$\mathrm{OH})$ to the silicate glass surface. DNA probes are typically printed in controlled patterns on microarray glass surfaces using robotic spotting and in-situ synthesis. ${ }^{1,2}$ In robotic spotting, the array is made by printing millions of spots on the glass surface, each from a controlled volume droplet of prepared DNA probe solution, hence the general name of spotting. The covalent attachment of the DNA probe is typically done in a post-spotting processing step. In conventional DNA spotting the printed DNA spots have a diameter in the range of 100-200 $\mu \mathrm{m}$. In in-situ synthesis, the oligos are built up base-by-base on the surface by the covalent reaction between the 5' hydroxyl group of the sugar of the last nucleotide to be attached and the phosphate group of the next nucleotide. The formation of probe oligos on microarray surfaces using both methods results in tethering one end of the probe oligo to the surface, which affects the duplex formation with a target in the sample solution as the bases nearest the surface are less accessible than those farthest away. The incorporation of spacer (linker) molecules that separate the oligo from the glass surface has been used to overcome the limitation of base accessibility for the entire sequence. The hybridization yields increased by up to two orders of magnitude with use of linkers. ${ }^{2-4}$ However, the length of the linker affects the duplex hybridization process and beyond an optimum linker length the hybridization yield reduces as the DNA strand may coil, thus making the probe less accessible to the complementary target. ${ }^{2}$ For DNA hybridization, the binding specificity also depends on the ionic strength (IS) of the hybridization buffer, where an $I S \sim 160$ $\mathrm{mM}$ that is comparable with a physiological sample medium is preferred for high hybridization efficiency.

A suitable probe layer construction, which is characterized by a good target density, compatible linker length, as well as length of probe molecules, are all fundamental and need to be designed for the specific surface transduction mechanism. In this thesis, Si-NW field-effect transistor (FET) biosensors are used to detect the electronic charge of target DNA hybridized to the sensor surface. Si-NW FET sensors measure surface potential changes on the sensor surface that originate from changes in the surface charge density due to changes in intrinsic charge of attached DNA duplexes. The surface potential change is determined from measurements of conductance change as described in Chapter 
5. The Si-NW sensor surface typically consists of a thin silicon dioxide $\left(\mathrm{SiO}_{2}\right)$ layer, which is chemically similar to the glass surfaces used for DNA microarrays, and therefore, the previously described techniques can be directly applied. However, in the case of electronic detection with Si-NW sensors, the high $I S$ required for efficient DNA hybridization is problematic for the detection of the electronic charge present in the DNA molecule, which requires short linker lengths $(\sim 1 \mathrm{~nm})$, such that the Debye screening at the sensor surface can be minimized. Since low ionic strength hybridization buffers are not suitable for high efficiency DNA-DNA duplex formation, we use a synthetic peptide nucleic acid (PNA) probe, a mimic of oligos with a charge-neutral peptide backbone that is reported to have low equilibrium dissociation constants to complementary DNA in low ionic strength buffers that have a relatively large Debye length; the DNA-PNA duplex formation is less dependent on the $I S$ of the hybridization buffer compared to DNA-DNA duplex formation. ${ }^{5-7}$

A common approach is to use direct attachment of DNA probes to the $\mathrm{Si}-\mathrm{NW} \mathrm{SiO}_{2}$ surface by tethering it covalently to an end-functionalized siloxane layer that is grafted onto the $\mathrm{SiO}_{2}$ surface, ${ }^{8-17}$ a hydrogen terminated silicon surface, ${ }^{18-20}$ or electrostatically to a positively charged poly-L-lysine layer on the surface. ${ }^{18,}{ }^{21}$ Typically 3-aminopropyltriethoxy silane (APTES) is used on the $\mathrm{SiO}_{2}$ surface, which is terminated with an amine group. The PNA has a thiol end group from the cysteine moiety and is attached to the amine of the APTES functionalized $\mathrm{SiO}_{2}$ surface using hetero-bi-functional cross-linker sulfosuccinimidyl-cyclohexane-carboxylate. ${ }^{22}$ The thiolated-amine attachment scheme is a very robust chemistry resulting in a surface probe density of $10^{13}$ molecules $\mathrm{cm}^{-2}$ of thiolated-DNA to the $\mathrm{SiO}_{2}$ surface. ${ }^{23}$ For APTES grafted on the $\mathrm{SiO}_{2}$ surface terminated with amine end-groups and attached to aminatedDNA is reported to have a reduced surface density $4 \times 10^{11}$ molecules $\mathrm{cm}^{-2}{ }^{24}$ The APTES based attachment scheme based on carboxylated PNA has been reported for Si-NW sensor probe surface preparation. ${ }^{10}$ APTES on the $\mathrm{SiO}_{2}$ surface with N-ethyl-N'-(3-(dimethylamino)propyl)-carbodiimide (EDC) and N-hydroxysuccinimide (NHS) results in vertical orientation of immobilized antibodies at their C-end. ${ }^{12}$ APTES followed by glutaraldehyde can couple antibodies via the $\mathrm{N}$-end resulting in horizontal orientation of the immobilized antibodies. ${ }^{12}$ The sulfo-NHS-SS-biotin attachment to APTES treated $\mathrm{SiO}_{2}$ surfaces is an one-step surface biotinylation method for streptavidin binding. ${ }^{22}$ The biotinylation of Si- 
NW surfaces has been reported using biotinyl p-nitrophenyl ester, with 4(dimethylamino)pyridine (DMAP) in pyridine (99.9\%), followed by avidin and biotinylated peptide nucleic acid for a PNA probe layer attachment. ${ }^{13}$ Biotinylation has also been performed using biotin-amidocaproyl labeled bovine serum albumin on Si-NWs. ${ }^{14}$ Using trimethoxy-silane-aldehyde, direct aminated PNA have been attached to the $\mathrm{SiO}_{2}$ surface. ${ }^{11,16}$ Silane aldehyde, followed by the reduction of the amine for biomolecule attachment via secondary amine bonds on $\mathrm{SiO}_{2}$ surface of $\mathrm{Si}-\mathrm{NWs}$ has been reported for a variety of biological target molecules detection, such as virus, ${ }^{15}$ tyrosine kinase, ${ }^{25}$ prostate specific antigen $^{17}$ and telomerase assay respectively. ${ }^{17}$ However, the basic siloxane coupling to $\mathrm{SiO}_{2}$ surfaces has associated problems of reduced hydrolytic stability, dependence on availability of hydroxyl binding sites on the $\mathrm{SiO}_{2}$, and the inherent risk of multilayer formation. ${ }^{26}$ Self-assembled monolayers of organophosphonates based on physisorption have been reported to be more stable in that aspect. ${ }^{27}$ The bio-functionalization of $\mathrm{SiO}_{2}$-terminated surfaces with organophosphate PNA oligonucleotides has been demonstrated for sensing with Si-NWs. ${ }^{26}$ Surface attachment directly to silicon surface without any intervening $\mathrm{SiO}_{2}$ is another approach to probe layer preparation with a motivation to keep the probe layer closer to the silicon transducing surface for Si-NW sensing applications. Direct molecular attachment to silicon is obtained by hydrogen termination of silicon following hydrosilylation. Si(111) surface is preferred for the covalent alkylation of organic monolayers due to the surface atomic arrangement, resulting in densely packed layers with low density of dangling bonds. For top down fabrication of Si-NWs mostly 111-plane of silicon is accessible for functionalization due to plane dependent etching method of formation of Si-NWs. ${ }^{28}$ Hydrogen terminated Si-NWs have been prepared after prior removal of native $\mathrm{SiO}_{2}$ from the $\mathrm{Si}-\mathrm{NW}$ surface followed by gas phase hydrosilylation. ${ }^{18}$ A stable $\mathrm{H}-\mathrm{Si}$ surface is formed, where the C-Si monolayer is prepared with a suitable precursor involving free radical initiation using ultraviolet (UV) irradiation, heating, electrochemical reaction, transition metal complexes, or Lewis acid catalysts respectively. ${ }^{19}$ Hydrosilylation has been used for PNA attachment using the homo-bifunctional cross-linker glutaraldehyde to bind aminated-PNA. ${ }^{20}$ Also non-covalent approach of probe layer formation on $\mathrm{SiO}_{2}$ based on electrostatic attraction of polymers on $\mathrm{SiO}_{2}$ due to inherently negative charge of $\mathrm{SiO}_{2}$ at neutral $\mathrm{pH}$ is implemented for probe layer formation. 
Poly-lysine is positively charged at $\mathrm{pH} 7$ and is used to attach electrostatically to $\mathrm{SiO}_{2}$ followed by negatively charged DNA. This method of non-covalent electrostatic attachment has been used to functionalize silicon surfaces with DNA probe layer for hybridization detection in horizontal fashion in physiological buffers. ${ }^{18,21}$

In this chapter we have characterized two surface attachment schemes for the formation of the PNA probe layer on a $\mathrm{SiO}_{2}$ surface using trimethoxy silane aldehyde and APTES. The surface density has been determined with both radioactive and fluorescence labeled assays. Since PNA probes are known to non-specifically adsorb to glass surfaces, ${ }^{29,30}$ we have performed experiments to confirm this effect, as well as non-specific PNA adsorption tests for silicon nitride $\left(\mathrm{Si}_{3} \mathrm{~N}_{4}\right)$, polyimide, and poly-dimethyl-siloxane (PDMS), which are all commonly used microfabrication materials. Since the non-specific adsorption of PNA is significant, we have investigated the printing of PNA probes in localized regions on the sensor surface only using a commercially available small volume spotting printer (Nano eNabler, Bioforce Nanosciences).

\subsection{Experimental}

\subsubsection{Chemicals}

All reagents and solvents used were of analytical grade and used without further purification. 3-amino-propyl-triethoxy-silane (APTES), tris-ethanolamine (TEA) hydrochloride and sodium cyanoborohydride $\left(\mathrm{NaCNBH}_{3}\right)$ were purchased from Sigma Aldrich. Trimethoxysilane aldehyde was purchased from United Chemical Technologies. Sulfo-succinimidyl-4-(N-maleimidomethyl)cyclohexane- 1-carboxylate (sulfo-SMCC) was purchased from Pierce. The poly-dimethyl-siloxane (PDMS) pre-polymer (Sylgard) was purchased from Dow Corning. Purified 15-mer peptide nucleic acid (PNA) molecules were purchased from Panagene, Korea, and the DNA oligomers were purchased from Eurogentec. The sequences of the PNA and DNA used have been tabulated in Table 1.

Table 1. Nucleic acid sequences used for all experiments.

\begin{tabular}{|c|c|}
\hline Molecule/Complex & Sequence \\
\hline PNA & Cys-TGT-ACA-TCA-CAA-CTA-NH \\
\hline Complementary (M0) DNA & 5'-TAG-TTG-TGA-TGT-ACA-3' \\
\hline Non-complementary (NC) DNA & 5'-ATC-AAC-ACT-ACA-TGT-3' \\
\hline
\end{tabular}




\subsubsection{Radiolabeling of oligonucleotides}

The DNA was radiolabeled with $\left(\gamma-{ }^{32} \mathrm{P}\right)$-ATP at the 5 , end using a T4 polynucleotide kinase (T4 PNK) as shown in Scheme 1, using a protocol from New England Biolabs. ${ }^{25}$ The T4 polynucleotide kinase was purchased from Sigma (10 units $\left.\mu 1^{-1}, 500 \mathrm{UN}\right),\left(\gamma_{-}{ }^{32}\right)$-ATP (BLU002250UC, $10 \mathrm{Ci} \mathrm{mmol}^{-1}, 2$ $\mathrm{mCi} \mathrm{ml}^{-1}, 250 \mu \mathrm{Ci}$ ). The $1 \times \mathrm{T} 4 \mathrm{PNK}$ buffer consists of $70 \mathrm{mM}$ Tris- $\mathrm{HCl}, 10 \mathrm{mM}$ $\mathrm{MgCl}_{2}$, and $5 \mathrm{mM}$ DTT at $\mathrm{pH} 7.6$ Briefly, the labeling process consisted of a 50 pico mole DNA sample in a $50 \mu \mathrm{l}$ reaction volume that was reacted with 20 enzyme units in the $1 \times \mathrm{T} 4 \mathrm{PNK}$ buffer for $1 \mathrm{hr}$ at $37^{\circ} \mathrm{C}$, and followed by direct ethanol precipitation of the radiolabeled DNA. All radiolabeling experiments were done under adequate radiation protection in a radioactive material handling certified laboratory (BL4 laboratory). Precipitated radiolabeled oligomers were washed with $70 \%$ ethanol three times until the Geiger counter measuring the signal was constant, which indicates that non-reacted isotope was washed away and only labeled probe was collected.

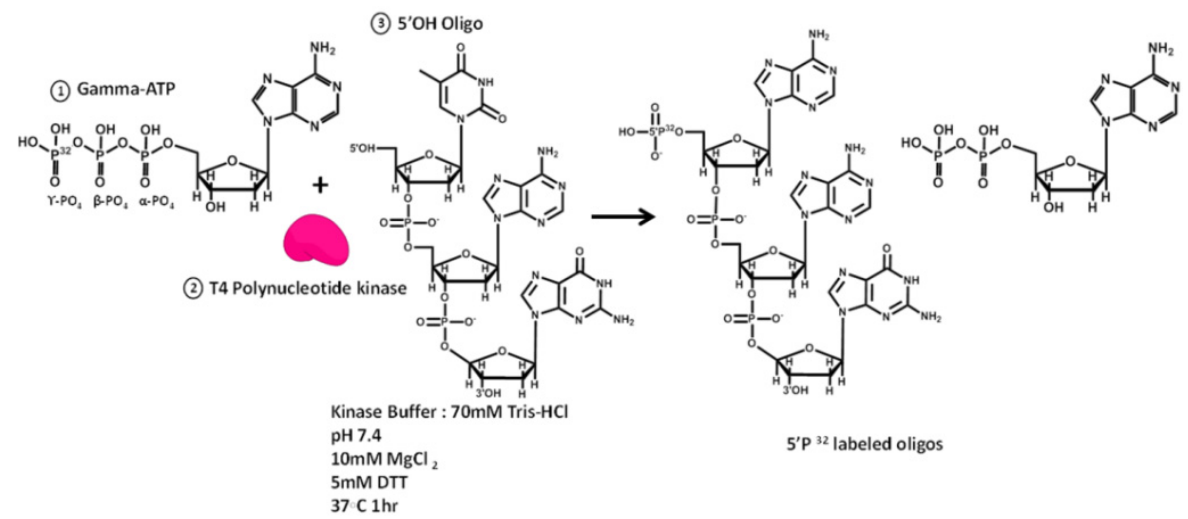

Scheme 1. Enzymatic radiolabeling of DNA at the 5 , end with a single ${ }^{32} \mathrm{P}$ with a $\gamma$ ATP $-{ }^{32} \mathrm{P}$ substrate.

\subsubsection{Probe attachment to $\mathrm{SiO}_{2}$ surface}

$\mathrm{SiO}_{2}$ samples with $1 \mathrm{~cm}^{2}$ surface area were used for the surface probe density studies with fluorescently labeled PNA (PNA-Cy5) and radiolabeled DNA oligomers. The probes were covalently attached to the $\mathrm{SiO}_{2}$ surface in a $5 \mathrm{~mm}$ diameter reaction vessel formed from a $4 \mathrm{~mm}$ thick PDMS layer to avoid droplet evaporation effects during immobilization. PDMS well chips were cut out with a knife and punched (5 mm, Harris-Uni-Core) from a PDMS (10:1 PDMS to 
curing agent ratio) slab cured on a blank silicon wafer. The PDMS wells were cleaned by sonication in ethanol. A $50 \mu \mathrm{l}$ probe solution was incubated in the PDMS-SiO 2 well chip in a humidity chamber. Subsequent reaction and washing steps were done inside the reaction vessel. Two types of attachment chemistry were followed, using two different silanes and end groups on $\mathrm{SiO}_{2}$ that bind aminated and thiolated probes.

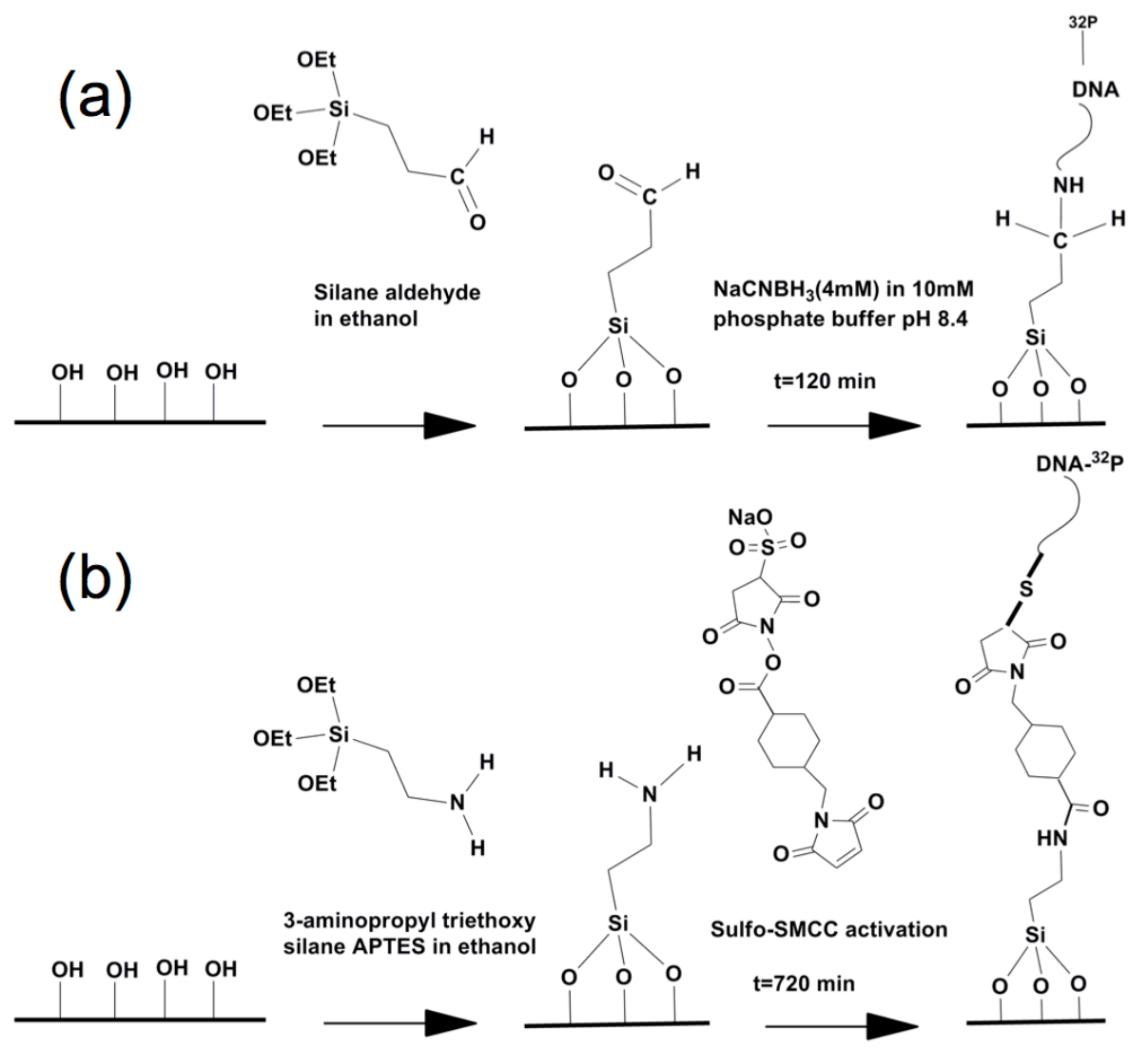

Scheme 2. Schematic of PNA attachment (a) Silane aldehyde-aminated-DNA (b) APTES-sulfo-SMCC-thiolated-DNA.

Thiolated probes were coupled to APTES treated $\mathrm{SiO}_{2}$ surfaces. Briefly, piranha cleaned $\mathrm{SiO}_{2}$ surfaces were treated with 2\% APTES in 95\% ethanol for $1 \mathrm{hr}$, washed in ethanol and heated to $120^{\circ} \mathrm{C}$ for $15 \mathrm{~min}$. APTES coated $\mathrm{SiO}_{2}$ is activated with $5 \mathrm{mM}$ hetero-bifunctional cross-linker sulfo-SMCC in $10 \mathrm{mM}$ TEA buffer $\mathrm{pH} 7.2$ for $1 \mathrm{hr}$ yielding a maleimide terminated surface to couple 1 $\mu \mathrm{M}$ thiolated probes in TEA buffer $\mathrm{pH} 7.2$ for $12 \mathrm{hrs}$ in a humidity chamber. 
Aminated probes were functionalized to silane aldehyde treated $\mathrm{SiO}_{2}$ surfaces. Briefly, piranha cleaned $\mathrm{SiO}_{2}$ surfaces were treated with $2 \%$ silane aldehyde in $95 \%$ ethanol for $1 \mathrm{hr}$, washed in ethanol, and heated to $120^{\circ} \mathrm{C}$ for $15 \mathrm{~min}$. Silane aldehyde conjugated to $\mathrm{SiO}_{2}$ surfaces was reacted with $1 \mu \mathrm{M}$ aminated probe with the $4 \mathrm{mM} \mathrm{NaCNBH}$ reducing agent in $10 \mathrm{mM}$ phosphate buffer $\mathrm{pH} 8.4$ for 2 hrs. The silane aldehyde on $\mathrm{SiO}_{2}$ surfaces is reduced to form a secondary amine with the aminated probe. A Phosphorus imager (Storm 860, Molecular Dynamics) was used for radioisotope detection from radiolabeled and sonicated surfaces. Since PNA cannot be radiolabeled, the probe surface attachment density of the aminated and thiolated probes has been estimated using radiolabeled oligomers, as well as fluorescence (aminated: Am-PNA-Cy5 and thiolated: Th-PNA-Cy5). Fluorescent measurements were performed using the Bio-Rad Molecular FX scanner.

\subsubsection{Non-specific adsorption of PNA}

In addition to the $\mathrm{SiO}_{2}$ surfaces, other surfaces including silicon nitride $\left(\mathrm{Si}_{3} \mathrm{~N}_{4}\right)$, polyimide (PI), and PDMS have also been tested for non-specific adsorption of probes after washing and sonication. DNA was allowed to hybridize to the

surface functionalized PNA probes for $1 \mathrm{hr}$ in static condition and in separate experiments for $10 \mathrm{~min}$ in a PDMS micro-channel with a flow rate of $7 \mu 1 \mathrm{~min}^{-1}$. For static hybridization (PDMS well based method) 10:1 PDMS was cured on a flat silicon wafer, then small area $\left(1 \mathrm{~cm}^{2}\right)$ PDMS squares were cut out and punched with a punching tool (5 mm diameter, Harris Uni-Core). The square PDMS pieces with punched holes are cleaned by sonication in ethanol, and followed by assembly on pre-cut $\mathrm{SiO}_{2}$ pieces to form the hybridization vessel.

\subsubsection{Small volume molecular printing}

The small volume molecular printer (MP) uses an etched silicon cantilever that resembles a quill type of printing element, called surface patterning tool (SPT), which is similar to a conventional cantilever that is used for atomic force microscopy with the exception of a microchannel that extends from reservoir along the length of the cantilever to an opening at the tip of the cantilever, shown schematically in Fig. 2. The printing works by capillary action, where the sample solution in the filled reservoir wicks down the microchannel and stops at the tip of the cantilever due to surface tension, which forms a sample meniscus 

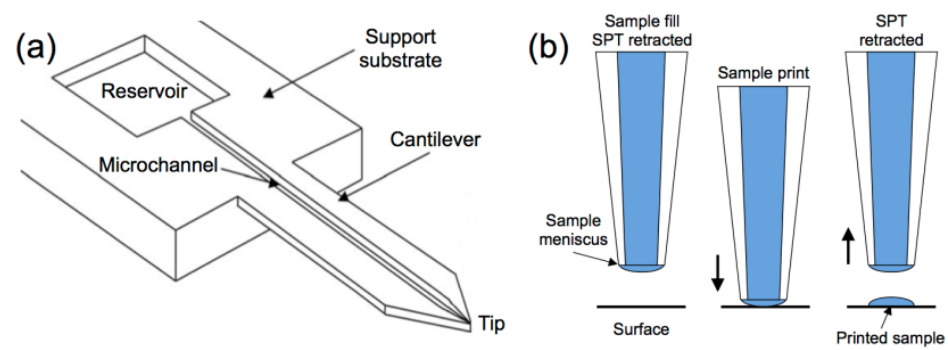

Figure 2. (a) Schematic of a SPT used in the molecular printer (b) Basic printing procedure that begins with the sample wicking to the end of the SPT in the form of a meniscus and the subsequent soft contact of the meniscus with the surface that forms the droplet on the surface. (Adapted from Bioforce Nanosciences)

at the cantilever tip, as shown in the printing sequence in Fig 2(b). We use a commercially available MP (Nano eNabler, Bioforce Nanosciences), which uses AFM-like SPTs. ${ }^{31}$ There have been reports of using this MP instrument for patterning spots with diameters below $1 \mu \mathrm{m},{ }^{32-34}$ however, we print droplets 8-12 $\mu \mathrm{m}$ in diameter. The MP has been reported for different types of applications. ${ }^{35-}$ 37 Currently, three SPT tip widths are available: $10 \mu \mathrm{m}, 30 \mu \mathrm{m}$ and $60 \mu \mathrm{m}$. Examples of rounded-end and sharp-ended tips are shown in Fig. 3. The SPT is reusable. Prior to each experiment, the SPT is cleaned using a UV-ozone treatment (UV/Ozone ProCleaner Plus, Bioforce Nanosciences) for $30 \mathrm{~min}$.

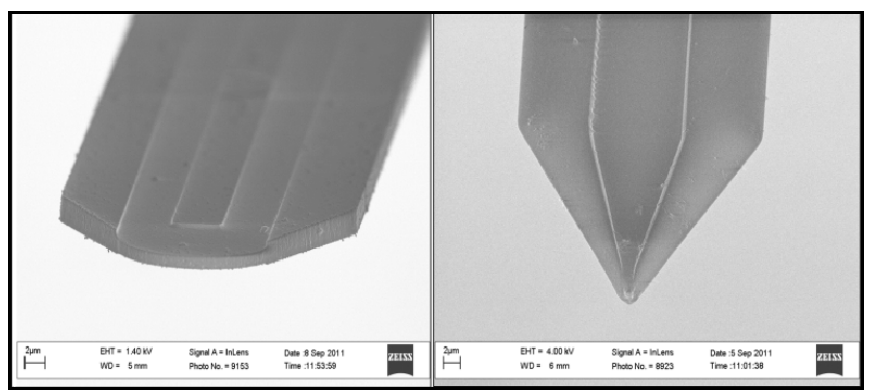

Figure 3. Different SPT Left: blunt tip Right: sharp tip.

The SPT holder fixed with the SPT is mounted into the instrument and the SPT aligned to the surface and calibrated for printing using the software interface. A $0.5 \mu \mathrm{l}$ printing solution is loaded into the rectangular reservoir, as shown in Fig. 2(a), after mounting an SPT onto a SPT holder. The MP has an internal humidifier to control the sample evaporation rate inside the chamber, which is important with the small volumes involved $(\sim 100 \mathrm{fL})$. Typically, 
glycerol is also added to the printing "ink", which retains water in the droplets. Initially, we demonstrated the printing of fluorescein-NHS. The printing ink consists of $1 \times$ PBS buffer pH 7.4 with $10 \%$ glycerol and $0.2 \%$ Tween 20 . In order to print fluorescein-NHS, the $1 \mathrm{~cm}^{2}$ area $\mathrm{SiO}_{2}$ surfaces were silanated with APTES and used for printing fluorescein-NHS. The humidity in the MP is maintained at 50\% and droplet diameters between 10-12 $\mu \mathrm{m}$ were obtained. Trimethoxy silane aldehyde was used for silanization of $\mathrm{SiO}_{2}$ surface for printing IgG and PNA via the aldehyde-amine coupling. The printing ink in that case consisted of $10 \mathrm{mM}$ phosphate buffer $\mathrm{pH} 8.4,4 \mathrm{mM} \mathrm{NaCNBH}, 10 \%$ glycerol (w/v), 0.2\% Tween 20, and IgG and PNA. The surfactant was added to make flat droplets to minimize the effect of border effects of the droplets formed, during functionalization.

\subsubsection{Printing in small areas}

In addition to printing on flat $\mathrm{SiO}_{2}$ surfaces, printing was also performed in $\mathrm{SiO}_{2}$ surfaces within patterned $200 \mu^{2}$ area windows in the polyimide insulation layer of Si-NW chips. These windows had a pair of Si-NWs $10 \mu \mathrm{m}$ in length and spaced by a distance of $10 \mu \mathrm{m}$. Printing was possible using the SPT (10S, Bioforce Nanosciences) inside the polyimide window to print on single Si-NW individually. The chemistry involved in this case was aldehyde-amine coupling and the printing ink comprised of $10 \mathrm{mM}$ phosphate buffer $\mathrm{pH} 8.4,4 \mathrm{mM}$ $\mathrm{NaCNBH}_{3}, 10 \%$ glycerol (w/v), $0.002 \%$ Tween 20 and 30\% humidity. The smaller amount of surfactant and less humidity allowed less spreading of droplets and droplet diameter around $8 \mu \mathrm{m}$ can be reliably printed. The blocking of surfaces with ethanolamine was followed after surface functionalization.

\subsection{Results and Discussions}

\subsubsection{Surface probe attachment density}

We have evaluated two different surface functionalization schemes for the attachment of PNA and DNA probes on $\mathrm{SiO}_{2}$ surfaces. Aminated DNA probes $\left(A m-D N A P^{32}\right)$ are attached to the $\mathrm{SiO}_{2}$ surface via reduction of the silane aldehyde surface groups with $\mathrm{NaCNBH}_{3}{ }^{16}$ and thiolated DNA probes (Th-DNA$\mathrm{P}^{32}$ ) are attached to $\mathrm{SiO}_{2}$ using maleimide activation on 3-amino-propyltriethoxy-silane (APTES) covered $\mathrm{SiO}_{2}$ surface. The Am-DNA-P ${ }^{32}$ attachment provides a probe surface density of $N_{\mathrm{s}} \sim 10^{10}$ molecules $\mathrm{cm}^{-2}$ and the Th-DNA-P ${ }^{32}$ 
attachment provides a probe surface density $N_{\mathrm{s}} \sim 5 \times 10^{12}$ molecules $\mathrm{cm}^{-2}$, which is in agreement with reported values of surface probe density for the respective attachment schemes. $^{23,38}$

Table 2. Surface probe density.

\begin{tabular}{|c|c|}
\hline Probe molecule & $\begin{array}{c}\text { Surface density } \times 10^{12} \\
\text { molecules } \mathrm{cm}^{-2}\end{array}$ \\
\hline Am-DNA-P & 0.01 \\
\hline Th-DNA-P & 4.6 \\
\hline Am-PNA-Cy5 & 0.5 \\
\hline Th-PNA-Cy5 & 1.2 \\
\hline
\end{tabular}

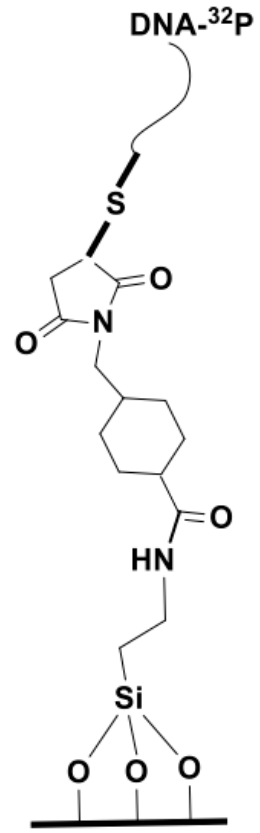

(a)
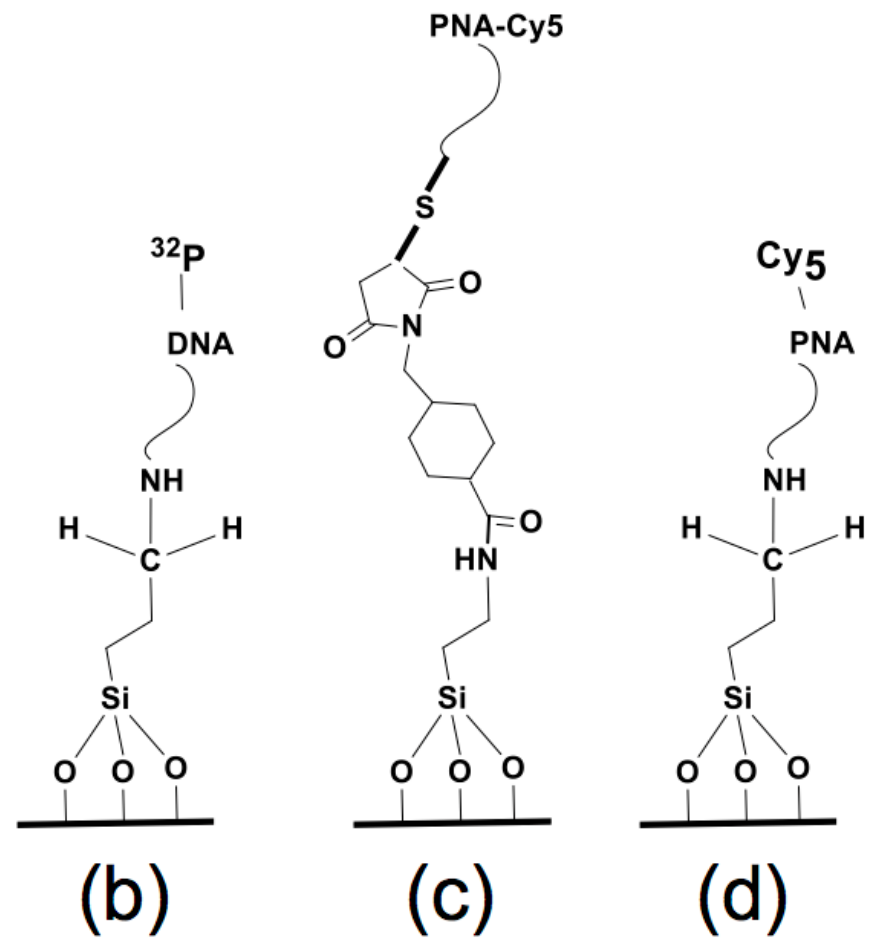

Figure 4. Summary of probe attachment structures. (a) Radiolabled DNA- $\mathrm{P}^{32}$ attached via thiolated scheme (b) Radiolabeled DNA- $\mathrm{P}^{32}$ attached via aminated scheme (c) Fluorecent PNA-Cy5 attached via thiolated scheme (d) Fluorescent PNA-Cy5 attached via aminated scheme.

Although the Th-DNA attachment scheme requires a long surface preparation time of 8-12 hours, ${ }^{23}$ compared to the Am-DNA attachment scheme that requires 
3-4 hrs, ${ }^{16}$ the Th-DNA is the preferred attachment method for electronic biosensors as a larger $N_{\mathrm{s}}$ results in a larger transduced signal, ${ }^{21}$ and a surface density of $5 \times 10^{12}$ molecules $\mathrm{cm}^{-2}$ is reported to be well-suited for good hybridization efficiency. ${ }^{39,40}$ The surface attachment density of PNA attachment to $\mathrm{SiO} 2$ surfaces is evaluated using a $\mathrm{Cy} 5$ fluorescence label for both aminated attachment (Am-PNA-Cy5) and thiolated attachment (Th-PNA-Cy5). The surface density for Am-PNA-Cy5 is Ns $\sim 0.5 \sim 10^{12}$ molecules $\mathrm{cm}^{-2}$ and for ThPNA-Cy5 is Ns $\sim 10^{12}$ molecules $\mathrm{cm}^{-2}$. The Am-DNA-P ${ }^{32}$ and Am-PNA-Cy5 probe densities differ by an order of magnitude and the discrepancy is related to the non-specific adsorption of Am-PNA to $\mathrm{SiO} 2$ surfaces, which is a known characteristic of PNA due to the uncharged backbone, ${ }^{30-41}$ and is critically important for surface hybridization assays

\subsubsection{Non-specific adsorption of PNA on surfaces}

To confirm the non-specific adsorption of the different PNA attachment schemes to the $\mathrm{SiO}_{2}$ surfaces, PNA was incubated on $\mathrm{SiO}_{2}$ surfaces without a crosslinking/reducing agent, and subsequently imaged using the radioactive and fluorescence labels, as previously described. The Am-PNA was incubated on $\mathrm{SiO}_{2}$ surfaces functionalized with silane aldehyde without any $\mathrm{NaCNBH}_{3}$ coupling buffer, and Th-PNA incubated on the $\mathrm{SiO}_{2}$ surface treated with APTES without cross-linking. Both surfaces were sonicated in deionized water for 15 min. and exposed to complementary DNA- ${ }^{32}$ for hybridization. Each surface was subsequently washed with a $1 \mathrm{M} \mathrm{NaCl}$ post-hybridization buffer and subsequently imaged.

Table 3. Non-specific adsorption of Am-PNA and Th-PNA to $\mathrm{SiO}_{2}$ surfaces.

\begin{tabular}{|c|c|}
\hline Complex & $\begin{array}{c}\text { Surface density } \\
\times 10^{12} \text { molecules } \text { cm }^{-2}\end{array}$ \\
\hline Am-PNA-DNA-P & 0.4 \\
\hline Am-PNA-Cy5 & 0.4 \\
\hline Th-PNA-Cy5 & 0.02 \\
\hline
\end{tabular}

The Am-PNA-DNA hybridization density is $0.4 \times 10^{12}$ molecules $\mathrm{cm}^{-2}$, which is the same order of magnitude as the previous surface density measurements of Am-PNA-Cy5 and clearly confirms the non-specific adsorption of the Am-PNA to the $\mathrm{SiO}_{2}$ surfaces. ${ }^{41}$ The surface density of Th-PNA non-specifically adsorbed to the APTES coated $\mathrm{SiO}_{2}$ is an order of magnitude less than the covalently 
coupled Th-PNA probe and can be explained by the fact that an APTES coated $\mathrm{SiO}_{2}$ surface is slightly positively charged due to the protonated amine groups at $\mathrm{pH}$ 7. It has been reported that poly-lysine and poly-aspartate differentially attach electrostatically to hafnium oxide deposited on $\mathrm{SiO}_{2}$ due to same principle of amine protonation of amines in poly-lysine, and de-protonation of aspartates at $\mathrm{pH}>7 .^{42}$ The non-specific adsorption results are summarized in Table 3.

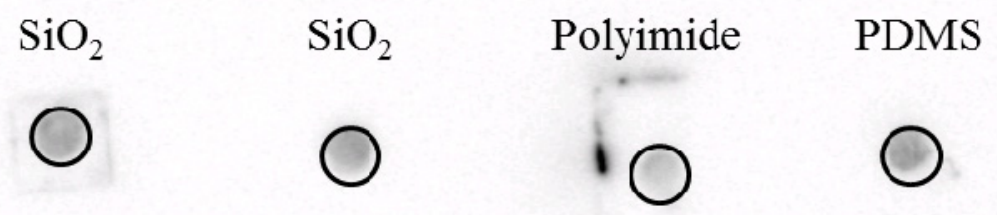

Figure 5. Non-specifically attached probe PNA hybridized to DNA-P ${ }^{32}$ on different surfaces.

Based on these observations we evaluated other material surfaces commonly used for biosensor manufacturing, including silicon nitride $\left(\mathrm{Si}_{3} \mathrm{~N}_{4}\right)$, polyimide (PI) and poly-dimethyl-siloxane (PDMS), by hybridizing DNA-P ${ }^{32}$ to surfaces that have been incubated with Am-PNA probe molecules. Following the hybridization step, the surfaces are washed with a high ionic strength wash buffer and sonicated for $15 \mathrm{~min}$. The Am-PNA probes were directly incubated in deionized water on the respective surfaces without any treatment with silane aldehyde, APTES, or cross-linker.

Table 4. Non-specific probe and target densities on surfaces.

\begin{tabular}{|c|c|}
\hline Description & $\begin{array}{c}\text { Surface Density } \\
\times 10^{12}{\text { molecules } \mathrm{cm}^{-2}}^{-2}\end{array}$ \\
\hline Am-PNA:DNA-P ${ }^{32}$ on $\mathrm{Si}_{3} \mathrm{~N}_{4}$ & 0.4 \\
\hline Am-PNA:DNA-P ${ }^{32}$ on polyimide & 0.2 \\
\hline Am-PNA:DNA-P ${ }^{32}$ on PDMS & 4 \\
\hline DNA- $\mathrm{P}^{32}$ on $\mathrm{SiO}_{2}$ & 0.2 \\
\hline DNA- $\mathrm{P}^{32}$ on $\mathrm{Si}_{3} \mathrm{~N}_{4}$ & 0.03 \\
\hline DNA-P $\mathrm{P}^{32}$ on polyimide & 0.1 \\
\hline DNA-P ${ }^{32}$ on PDMS & 0.02 \\
\hline
\end{tabular}

The surface density of DNA-P ${ }^{32}$ hybridized to PNA non-specifically adsorbed to the $\mathrm{Si}_{3} \mathrm{~N}_{4}$ surface is $0.4 \times 10^{12}$ molecules $\mathrm{cm}^{-2}$, to the PI surface is $0.2 \times 10^{12}$ molecules $\mathrm{cm}^{-2}$, and on the PDMS surface is $4 \times 10^{12}$ molecules $\mathrm{cm}^{-2}$, all of which are quite significant. We have also allowed only DNA-P ${ }^{32}$ to be incubated in 
PDMS well on each of the surfaces, to find out about non-specific adsorption of DNA. These values have been tabulated in Table 4.

\subsubsection{Printing}

Printing on flat $\mathrm{SiO}_{2}$ surfaces was obtained by printing fluorescein-NHS molecules in PBS buffer with glycerol and Tween 20. The $\mathrm{pH}$ of the buffer mixture containing glycerol and tween were confirmed to be $\mathrm{pH} 7.4$ prior to printing as that is crucial for the chemical reaction to take place. Phosphate buffer is quite stable with addition of glycerol. $\mathrm{pH}$ of other buffer like borate buffer (as mentioned in technical sheet for fluorescein-NHS) changed a lot on addition of glycerol and was not compatible to be used as printing ink mixture. The $\mathrm{SiO}_{2}$ was previously silanated with APTES monolayer and there was successful chemical attachment of the NHS functional group to the amine end group from APTES.

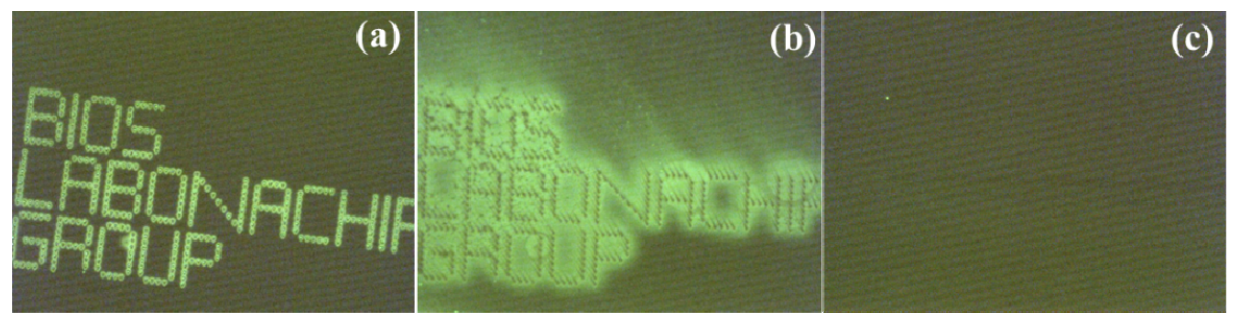

Figure 6. Fluorescein-NHS printed on APTES coated $\mathrm{SiO}_{2}$ surface (a) after printing and (b) after incubation and washing (c) control $\mathrm{SiO}_{2}$ surface without APTES coating, after washing.

However the NHS group is very reactive and during washing of the $\mathrm{SiO}_{2}$ surfaces post incubation, it spread and reacted with available amine groups as seen in the post-wash fluorescent image in Fig. 6(b). After confirming the amount of glycerol and surfactant for printing on the flat $\mathrm{SiO}_{2}$ surface, the same printing ink formula was extended to print other functional biomolecules, such as IgG and PNA using the chemistry of amine-aldehyde coupling with reducing agent sodium cyano-borohydride. 

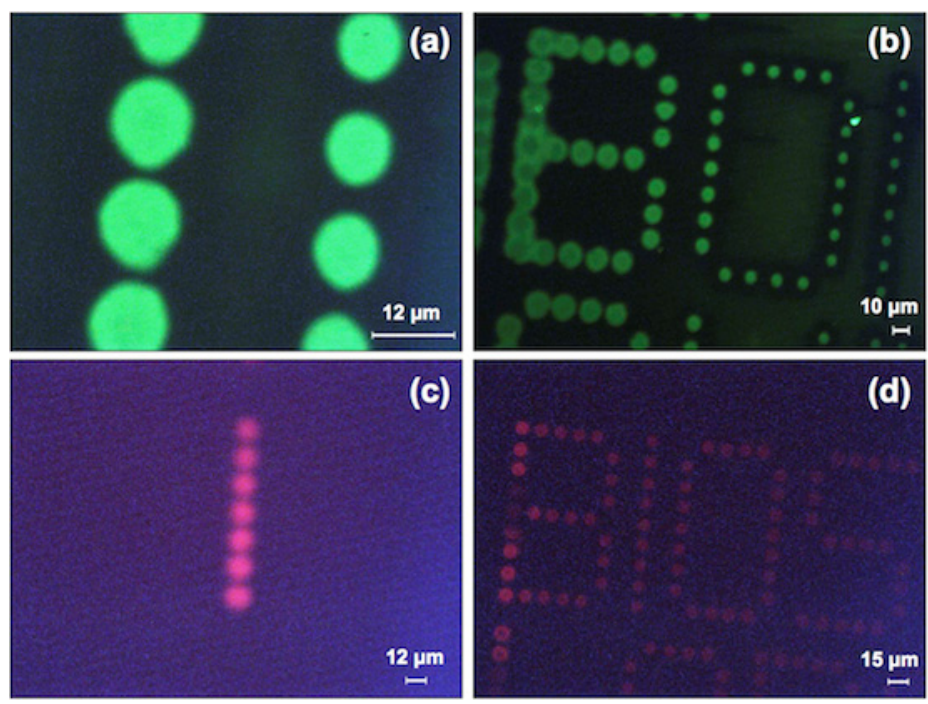

Figure 7. Examples of biomolecules printed on $\mathrm{SiO}_{2}$ surface terminated with silane aldehyde. (a) and (b) IgG printed and reacted with anti-IngG-Alex-Fluor-488, (c) and (d) PNA printed and hybridized with DNA-Cy5.

Figure 7 shows examples of IgG and PNA printed on surface. In Figs. 7(a) and Fig. 7(b), IgG has been printed on the $\mathrm{SiO}_{2}$ surface that is silanated with silane aldehyde linker and reacted with fluorescently labeled anti-IgGAlexa Fluor-488 (100:1 dilution). In Fig. 7(c) and Fig. 7(d), the $\mathrm{SiO}_{2}$ surface with cross-linked silane aldehyde layer has been reacted with aminated PNA and hybridized with complementary $10 \mu \mathrm{M}$ DNA-Cy5 in $1 \times$ PBS for $1 \mathrm{hr}$. All images were obtained after washing and drying the surfaces after all incubation steps with the appropriate fluorescent filter. The PNA probes have also been printed inside the polyimide window (area: $200 \mu \mathrm{m}^{2}$ ) by controlling the printink ink with a smaller percentage of surfactant and reduced humidity. Fig. 8(a) shows optical image of the printing on a single Si-NW inside an open polyimide window on a Si-NW chip. Fig. 8(b) is the fluorecent image post incubation and washing of the same surface as in Fig. 8(a). 


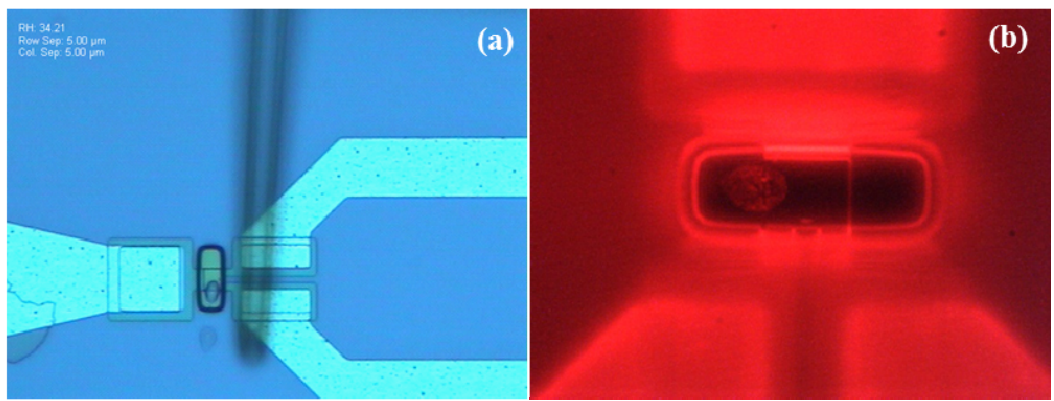

Figure 8. (a) Optical image of single droplet of PNA-Cy5 printed inside a polyimide window on a single Si-NW (b) Fluorescent image after incubation and washing.

We also attempted to block the surface with ethanolamine after attachment of the PNA, however, all of the PNA appeared to be depleted from the surface as can be seen by comparing Fig. 9(a) with Fig. 9(b). Thereafter, no blocking was performed after PNA functionalization on the $\mathrm{SiO}_{2}$ surface.
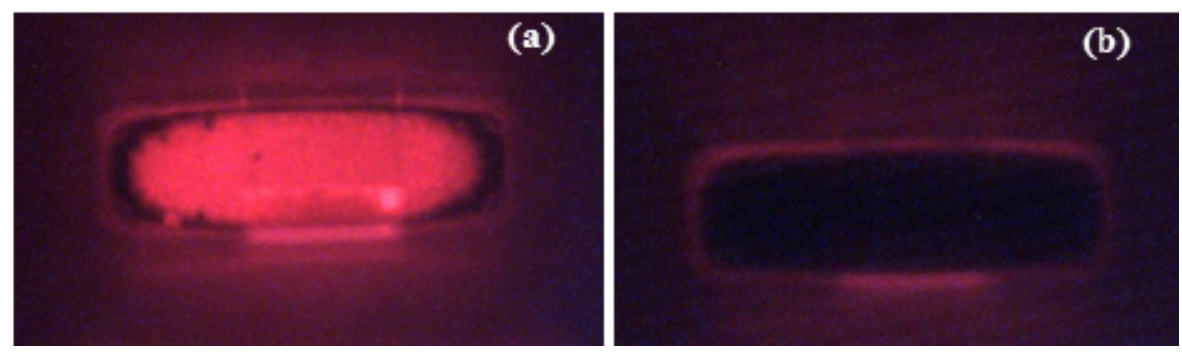

Figure 9. (a) Fluorescent image inside polyimide window treated with PNA-Cy5 after incubation and washing after printing and (b) after incubation and washing of the same window treated with ethanolamine.

\subsection{Conclusion}

In summary, PNA-DNA hybridization on solid surfaces is promising for biosensor applications due to their stability and flexibility for synthesis into different configurations; however, careful consideration of the surface attachment scheme and surfaces is required for their use in practical applications. Furthermore, we have evaluated two commonly reported attachment schemes and report that a thiolated attachment scheme is preferred as it provides significantly larger probe surface density and negligible non-specific adsorption of PNA to most materials. Additionally, we demonstrate a localized functionalization with the aminated chemistry with $\mathrm{IgG}$, and PNA probe molecules with small droplet with diameters around $8 \mu \mathrm{m}$. 


\section{References}

1. V. G. Cheung, M. Morley, F. Aguilar, A. Massimi, R. Kucherlapati and G. Childs, Nature Genetics, 1999, 21, 15-19.

2. E. Southern, K. Mir and M. Shchepinov, Nature Genetics, 1999, 21, 5-9.

3. D. J. Duggan, M. Bittner, Y. Chen, P. Meltzer and J. M. Trent, Nature Genetics, 1999, 21, 10-14.

4. M. S. Shchepinov, S. C. Case-Green and E. M. Southern, Nucleic Acids Res, 1997, 25, 1155-1161.

5. M. Egholm, O. Buchardt, L. Christensen, C. Behrens, S. M. Freier, D. A. Driver, R. H. Berg, S. K. Kim, B. Norden and P. E. Nielsen, Nature, 1993, $365,566-568$.

6. H. Park, A. Germini, S. Sforza, R. Corradini, R. Marchelli and W. Knoll, Biointerphases, 2007, 2, 80-88.

7. J. Liu, L. Tiefenauer, S. Tian, P. E. Nielsen and W. Knoll, Analytical Chemistry, 2006, 78, 470-476.

A. Poghossian, A. Cherstvy, S. Ingebrandt, A. Offenhäusser and M. J. Schöning, Sensors and Actuators, B: Chemical, 2005, 111-112, 470-480.

8. Poghossian, S. Ingebrandt, M. H. Abouzar and M. J. Schöning, Applied Physics A: Materials Science and Processing, 2007, 87, 517-524.

9. Gao, N. Lu, P. Dai, T. Li, H. Pei, X. Gao, Y. Gong, Y. Wang and C. Fan, Nano Letters, 2011, 11, 3974-3978.

10. Z. Gao, A. Agarwal, A. D. Trigg, N. Singh, C. Fang, C. H. Tung, Y. Fan, K. D. Buddharaju and J. Kong, Analytical Chemistry, 2007, 79, 3291-3297.

11. Vacic, J. M. Criscione, N. K. Rajan, E. Stern, T. M. Fahmy and M. A. Reed, Journal of the American Chemical Society, 2011, 133, 13886-13889.

12. W. Cai, J. R. Peck, D. W. Van Der Weide and R. J. Hamers, Biosensors and Bioelectronics, 2004, 19, 1013-1019.

13. Y. Cui, Q. Wei, H. Park and C. M. Lieber, Science, 2001, 293, 1289-1292.

14. F. Patolsky, G. Zheng, O. Hayden, M. Lakadamyali, X. Zhuang and C. M. Lieber, Proceedings of the National Academy of Sciences of the United States of America, 2004, 101, 14017-14022.

15. F. Patolsky, G. Zheng and C. M. Lieber, Nature Protocols, 2006, 1, 17111724.

16. G. Zheng, F. Patolsky, Y. Cui, W. U. Wang and C. M. Lieber, Nature Biotechnology, 2005, 23, 1294-1301. 
17. Y. L. Bunimovich, Y. S. Shin, W. S. Yeo, M. Amori, G. Kwong and J. R. Heath, Journal of the American Chemical Society, 2006, 128, 16323-16331.

18. M. N. Masood, S. Chen, E. T. Carlen and A. van den Berg, ACS Applied Materials and Interfaces, 2010, 2, 3422-3428.

19. G. J. Zhang, G. Zhang, J. H. Chua, R. E. Chee, E. H. Wong, A. Agarwal, K. D. Buddharaju, N. Singh, Z. Gao and N. Balasubramanian, Nano Letters, 2008, 8, 1066-1070.

20. J. Fritz, E. B. Cooper, S. Gaudet, P. K. Sorger and S. R. Manalis, Proceedings of the National Academy of Sciences of the United States of America, 2002, 99, 14142-14146.

21. E. Stern, R. Wagner, F. J. Sigworth, R. Breaker, T. M. Fahmy and M. A. Reed, Nano Letters, 2007, 7, 3405-3409.

22. L. A. Chrisey, G. U. Lee and C. E. O'Ferrall, Nucleic Acids Research, 1996, 24, 3031-3039.

23. V. Dugas, G. Depret, Y. Chevalier, X. Nesme and E. Souteyrand, Sensors and Actuators, B: Chemical, 2004, 101, 112-121.

24. W. U. Wang, C. Chen, K. H. Lin, Y. Fang and C. M. Lieber, Proceedings of the National Academy of Sciences of the United States of America, 2005, 102, 3208-3212.

25. Cattani-Scholz, D. Pedone, M. Dubey, S. Neppl, B. Nickel, P. Feulner, J. Schwartz, G. Absteiter and M. Tornow, ACS Nano, 2008, 2, 1653-1660.

26. E. L. Hanson, J. Schwartz, B. Nickel, N. Koch and M. F. Danisman, Journal of the American Chemical Society, 2003, 125, 16074-16080.

27. S. Chen, J. G. Bomer, W. G. Van der Wiel, E. T. Carlen and A. van den Berg, ACS Nano, 2009, 3, 3485-3492.

28. M. Masuko, Nucleic Acids Symposium Series, 2003, 3, 145-146.

29. A. Cattani-Scholz, D. Pedone, F. Blobner, G. Abstreiter, J. Schwartz, M. Tornow and L. Andruzzi, Biomacromolecules, 2009, 10, 489-496.

30. J. Xu, M. Lynch, J. L. Huff, C. Mosher, S. Vengasandra, G. Ding and E. Henderson, Biomedical Microdevices, 2004, 6, 117-123.

31. M. Lynch, C. Mosher, J. Huff, S. Nettikadan, J. Johnson and E. Henderson, Proteomics, 2004, 4, 1695-1702.

32. J. Ji, J. C. Yang and D. N. Larson, Biosensors and Bioelectronics, 2009, 24, 2847-2852. 
33. Y. Lou, L. M. Lunardi and J. F. Muth, IEEE Sensors Journal, 2010, 10, 617-620.

34. S. Nettikadan, K. Radke, J. Johnson, J. Xu, M. Lynch, C. Mosher and E. Henderson, Molecular and Cellular Proteomics, 2006, 5, 895-901.

35. S. R. Nettikadan, J. C. Johnson, S. G. Vengasandra, J. Muys and E. Henderson, Nanotechnology, 2004, 15, 383-389.

36. K. M. Radke, S. R. Nettikadan, J. C. Johnson, S. G. Vengasandra and E. Henderson, Analytical Biochemistry, 2004, 330, 350-352.

37. N. Zammatteo, L. Jeanmart, S. Hamels, S. Courtois, P. Louette, L. Hevesi and J. Remacle, Analytical Biochemistry, 2000, 280, 143-150.

A. W. Peterson, R. J. Heaton and R. M. Georgiadis, Nucleic Acids Research, 2001, 29, 5163-5168.

38. T. M. Herne and M. J. Tarlov, Journal of the American Chemical Society, 1997, 119, 8916-8920.

39. J. Weiler, H. Gausepohl, N. Hauser, O. N. Jensen and J. D. Hoheisel, Nucleic Acids Research, 1997, 25, 2792-2799.

40. N. M. Fahrenkopf, P. Z. Rice, M. Bergkvist, N. A. Deskins and N. C. Cady, ACS Applied Materials and Interfaces, 2012, 4, 5360-5368. 


\section{Chapter 7}

\section{Horizontal versus vertical PNA-DNA hybridization 5}

In this chapter we compare the PNA-DNA duplex hybridization characteristics of vertically tethered and new horizontally tethered PNA probes on solid surfaces. The horizontal 15-mer PNA probe has been synthesized with linker molecules attached at three locations ( $\gamma$-points) positioned along the PNA backbone that provides covalent attachment of the probe with the backbone aligned parallel to the surface, which is important for DNA hybridization assays that use electric field effect sensors for detection. A radiolabeling assay, and real-time surface plasmon resonance (SPR) biosensor are used to assess the probe surface density, non-specific binding, and DNA hybridization affinity, respectively, of the new PNA probe configuration. The estimated equilibrium dissociation constant $K_{\mathrm{D}} \sim 5 \mathrm{nM}$ indicates a sufficient hybridization affinity for many electronic biosensors that benefit from the horizontal alignment, which minimizes the effects of counter-ion screening.

\footnotetext{
${ }^{5}$ Adapted from: A. De, S. Souchelnytskyi, A. van den Berg, E.T. Carlen, Peptide nucleic acid (PNA)-DNA duplexes: Comparison of hybridization affinity between vertically and horizontally tethered PNA probes, ACS Appl. Mater. Interfaces, 2013, 5, 4607-4612.
} 


\subsection{Introduction}

Molecular assays based on the hybridization of target DNA to complementary DNA oligomer probes attached to a solid surface were first reported nearly four decades ago, ${ }^{1}$ and have since been applied extensively to the measurement of gene expression using microarrays ${ }^{2}$ and molecular diagnostics using biosensors. ${ }^{3}$ Stable DNA duplexes are readily formed in high ionic strength buffers due to the relaxation of the electrostatic repulsion of the complementary strands, which promotes inter-strand complementary base pairing. ${ }^{4,5,6}$ Synthetic DNA-like molecules, peptide nucleic acid (PNA), locked nucleic acid (LNA), and phosphoramidate morpholino (MORF) oligomers have been reported over the last several years for improved DNA and RNA hybridization performance. LNAs include a modification to the ribose sugar unit of the nucleotide that retains the negative charge from the phosphate backbone and provides highly specific RNA sequence recognition and do not suffer from aggregation problems typically associated with PNA. ${ }^{7,8}$ MORFs are uncharged probes for DNA hybridization, where 6-membered morpholino rings replace the ribose sugar, and a non-ionic phosphordiamidate linkage replaces the negatively charged phosphodiester backbone. The MORFs are reported to be better suited for gene expression applications since they can be synthesized with up to 70 nucleotides, ${ }^{9}$ compared to LNA and PNA. In this article, we are interested in PNA probes because of their synthesis flexibility, and specifically the incorporation of chiral units along the carbon chains of the molecule backbone that provide unique molecular configurations for functional group attachment and conjugation to solid surfaces. ${ }^{10,11,12}$
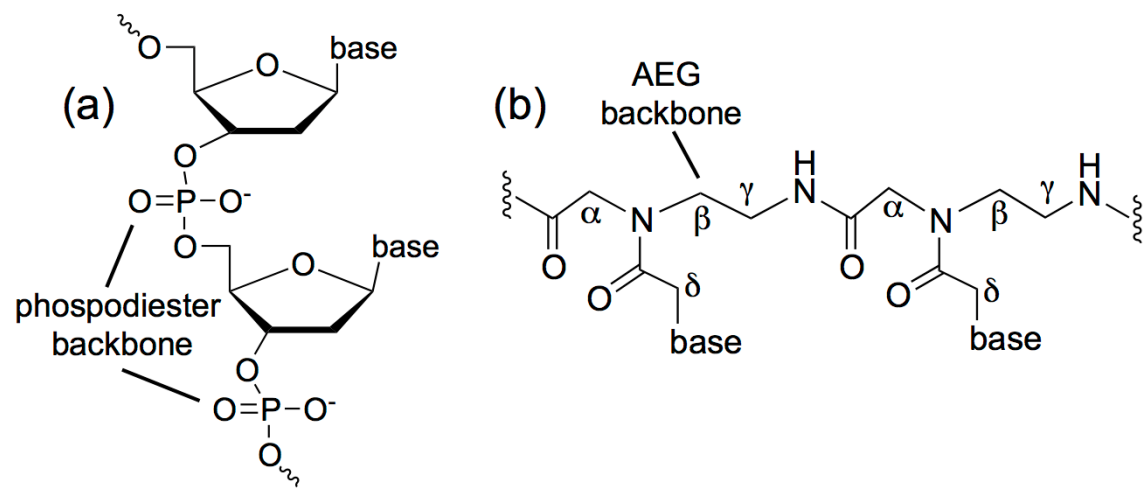

Figure 1. Backbone structures: (a) DNA (b) PNA. 
PNA probes, which form PNA-DNA complementary duplexes according to established Watson-Crick base pairing rules, have been reported to have higher affinity and sequence selectivity compared to conventional DNA-DNA duplex hybridization. ${ }^{13}$ PNA is an uncharged DNA mimic where the negatively charged phosphodiester backbone of DNA as shown in Fig. 1(a) is replaced with an achiral 2-amino-ethyl-glycine (AEG) backbone, shown in Fig. 1(b). ${ }^{14}$ The improvement in thermodynamic stability of the PNA-DNA duplex has been attributed to the lack of electrostatic repulsion in the backbone ${ }^{14}$ and another contribution may come from counter-ion release upon hybridization, as opposed to condensation taking place within DNA. ${ }^{15}$ In addition, the AEG-backbone is highly resistant to enzymatic degradation from nucleases and proteases that are widely used for regulating gene expression. ${ }^{16}$ Other applications for PNA include the identification of single nucleotide polymorphism, ${ }^{17}$ and isolation and purification of nucleic acids. ${ }^{18}$

Surface immobilized PNA probes are particularly important for biosensor applications due to the PNA-DNA duplex stability and their flexibility for synthesis into different configurations, especially for field-effect transistor (FET) sensors, such as silicon nanowire sensors ${ }^{19,20,21}$, which measure the intrinsic charge of hybridized complementary DNA to surface immobilized PNA probes on the gate-oxide sensor surface in the form of an increase in surface charge density. Conventional DNA and PNA probes have been attached to the gate-oxide surface using various chemical crosslinking strategies, which result in DNA or PNA probes extended vertically away from the sensor surface. For electronic biosensors, such as FET sensors that measure the net intrinsic electronic charge of the hybridized duplex on the sensor surface, there is a fundamental problem due to counter-ion screening at the gate-oxide surface $^{22,23,24}$ and counter-ion condensation of the negatively charged DNA, ${ }^{6}$ which can significantly reduce the net detectable surface charge. The length of the counter-ion screening layer, the Debye length $\lambda_{\mathrm{D}}$, is dependent on the ionic strength of the hybridization buffer - a high ionic strength results in a small $\lambda_{\mathrm{D}}$ and charge screening beyond $\sim 1-2 \mathrm{~nm}$ above the gate-oxide surface, ${ }^{22,23}$ which means that electronic charge extending beyond $\lambda_{\mathrm{D}}$ from the sensor surface will be effectively screened and will not be transduced into a measurable sensor signal.

Initial reports indicated that low ionic strength buffers, where $\lambda_{\mathrm{D}}$ is 
large enough to preclude counter-ion screening, did not negatively affect the PNA-DNA hybridization affinity, ${ }^{19,20}$ however, it was later reported that PNADNA hybridization kinetics favor high to moderate ionic strength buffers. ${ }^{25}$ Therefore, conventional vertically extended PNA-DNA duplex detection sensitivity is affected by counter-ion screening in a similar way as DNA-DNA hybridization. ${ }^{23}$ It has also been reported that the hybridization of DNA to surface adsorbed DNA probes with the backbone aligned parallel, or horizontal, to the sensor surface is more effective for the electronic detection of DNA compared to vertically tethered complementary probes as the electronic charge of the duplex is minimally screened by counter-ions in the hybridization buffer at the gate-oxide surface. ${ }^{26,27}$ However, previous reports used physically adsorbed DNA probes on the gate-oxide surface, whereas, covalently attached probe molecules are better suited for molecular assays. Our group has previously reported silicon nanowire chemical sensors for the electronic detection of sample $\mathrm{pH},{ }^{28}$ and biosensors for the measurement of PNA-DNA hybridization detection, where the PNA probe was attached to the silicon nanowire surface using a conventional vertical orientation, in low ionic strength buffer. ${ }^{21}$

Previous research on PNA backbone modification at the $\alpha$ and $\gamma$ positions Fig. 1(b) has been reported to increase the selectivity of complementary DNA recognition due to the presence of chiral centers. ${ }^{29}$ Furthermore, studies of $\gamma$ modified PNAs revealed that the introduction of a side chain in this position does not hinder formation of DNA and RNA duplexes; and the insertion of the modified PNA into a standard PNA oligomer induces structural organization and allows for an improvement in the binding affinity. ${ }^{10,30}$ In fact, the modification to the PNA backbone at the chiral $\gamma$ positions gives an inherent helicity to the PNA making it more rigid, thus easier to form a duplex with DNA. ${ }^{10}$ The $\gamma$ modifications to the backbone can also provide functional end groups for rotating the molecule such that the PNA probe can be covalently attached with horizontal alignment to the sensor surface using a conventional aldehyde amine reduction attachment scheme. ${ }^{31}$ Fig. 2 shows a comparison between a vertical PNA-DNA duplex with single end-point covalent PNA attachment Fig. 2(a) and a horizontal 

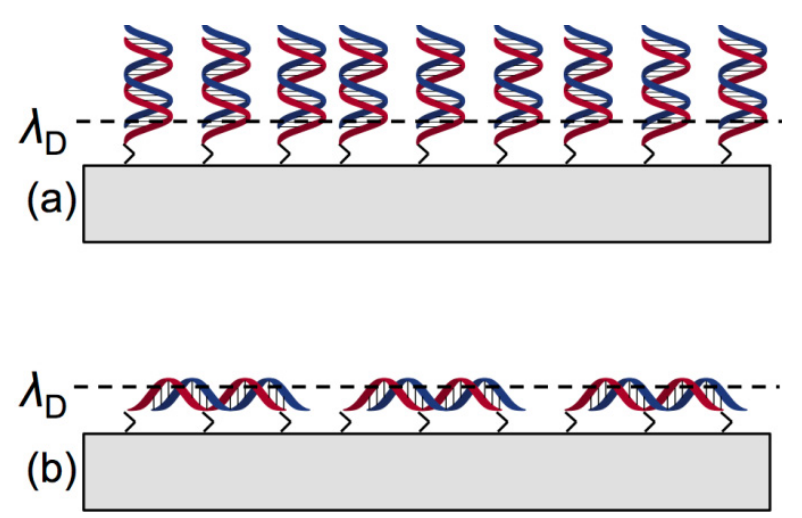

Figure 2. Qualitative PNA-DNA duplex hybridization formats and relation to the Debye length $\lambda_{\mathrm{D}}$. (a) vertical PNA probes with single end-point attachment (b) horizontal PNA probes with three $\gamma$ attachment points.

PNA-DNA duplex with a three covalent attachment points conjugated to the three $\gamma$ points in the PNA backbone and their relation to the Debye length $\lambda_{\mathrm{D}}$, Fig. 2(b). Therefore, hybridized PNA-DNA duplexes aligned parallel to the gateoxide surface will also be minimally affected by counter-ions in moderately high ionic strength buffers, which is required for fast and selective hybridization kinetics. Furthermore, the horizontal probe alignment does not limit the probe length, as is the case with the conventional vertically tethered probe.

\subsection{Experimental}

\subsubsection{Chemicals}

All reagents and solvents used were of analytical grade and used without further purification. 3-amino-propyl-triethoxy-silane (APTES), tris-ethanolamine (TEA) hydrochloride and sodium cyanoborohydride $\left(\mathrm{NaCNBH}_{3}\right)$ were purchased from Sigma Aldrich. Trimethoxysilane aldehyde was purchased from United Chemical Technologies. Sulfo-succinimidyl 4-(Nmaleimidomethyl)cyclohexane-1-carboxylate (sulfo-SMCC) was purchased from Pierce. The poly-dimethyl-siloxane (PDMS) pre-polymer (Sylgard, Dow Corning). Purified peptide nucleic acids (PNA) were purchased from Panagene, Korea, and the DNA oligomers were purchased from Eurogentec. The sequences of the probe PNAs and target DNAs used are tabulated in Table 1. 
Table 1. Sequence of PNAs and DNA used for experiments.

\begin{tabular}{|c|c|}
\hline Molecule & Sequence \\
\hline PNA & Cys-TGT-ACA-TCA-CAA-CTA-NH 2 \\
\hline Am- $\gamma$-PNA & $\begin{array}{c}\text { Ac-TGT*(AEEA)-ACA-TC*(AEEA)A- } \\
\text { CAA-C*(AEEA)TA }\end{array}$ \\
\hline $\begin{array}{c}\text { Th- } \gamma \text {-PNA } \\
\text { Ac-TGT*(C6-SH)-ACA-TC*(C6- } \\
\text { SH)A-CAA-C*(C6-SH)TA-3' }\end{array}$ \\
\hline $\begin{array}{c}\text { Complementary (M0) } \\
\text { DNA }\end{array}$ & 5'-TAG-TTG-TGA-TGT-ACA-3' \\
\hline $\begin{array}{c}\text { Non-complementary } \\
\text { (NC) DNA }\end{array}$ & 5'-ATC-AAC-ACT-ACA-TGT-3' \\
\hline
\end{tabular}

\subsubsection{Radiolabeling of oligonucleotides}

The DNA was radiolabeled with $\left(\gamma-{ }^{32} \mathrm{P}\right)$-ATP at the 5 , end using a T4 polynucleotide kinase (T4 PNK) using a protocol from New England Biolabs. ${ }^{25}$ The T4 polynucleotide kinase was purchased from Sigma (10 units $\mu 1^{-1}, 500$ $\mathrm{UN}),\left(\gamma^{-32}\right)$-ATP (BLU002250UC, $10 \mathrm{Ci} \mathrm{mmol}^{-1}, 2 \mathrm{mCi} \mathrm{ml}^{-1}, 250 \mu \mathrm{Ci}$ ). The $1 \times$ T4 PNK buffer consists of $70 \mathrm{mM}$ Tris- $\mathrm{HCl}, 10 \mathrm{mM} \mathrm{MgCl}_{2}$, and $5 \mathrm{mM}$ DTT at $\mathrm{pH}$ 7.6. Briefly, the labeling process consisted of a 50 picomole DNA sample in a $50 \mu 1$ reaction volume that was reacted with 20 enzyme units in the $1 \mathrm{X}$ T4 PNK buffer for $1 \mathrm{hr}$ at $37^{\circ} \mathrm{C}$, and followed by direct ethanol precipitation of the radiolabeled DNA. All radiolabeling experiments were done under adequate radiation protection in a radioactive material handling certified laboratory (BL4 laboratory). Precipitated radiolabeled oligomers were washed with $70 \%$ three times until the Geiger counter measuring the signal was constant. Constant signal indicated that non-reacted isotope was washed away and only labeled probe was collected.

\subsubsection{PNA probe surface attachment on $\mathrm{SiO}_{2}$}

$\mathrm{SiO}_{2}$ samples with $1 \mathrm{~cm}^{2}$ surface area were used for the surface probe density studies. The probes were covalently attached to the $\mathrm{SiO}_{2}$ surface in a $5 \mathrm{~mm}$ diameter reaction vessel formed from a $4 \mathrm{~mm}$ thick PDMS layer to avoid droplet evaporation effects during immobilization. PDMS well chips were cut out with a knife and punched (5 mm, Harris-Unicore) from a PDMS (10:1 PDMS to curing agent ratio) slab cured on a blank silicon wafer. The PDMS wells were cleaned by sonicating in ethanol. A $50 \mu \mathrm{l}$ probe solution was incubated in the PDMS$\mathrm{SiO}_{2}$ well chip in a humidity chamber. Subsequent reaction and washing steps were done inside the reaction vessel. Aminated probes were functionalized to 
silane aldehyde treated $\mathrm{SiO}_{2}$ surfaces. Briefly, piranha cleaned $\mathrm{SiO}_{2}$ surfaces were treated with $2 \%$ silane aldehyde in $95 \%$ ethanol for $1 \mathrm{hr}$., washed in ethanol, and heated to $120^{\circ} \mathrm{C}$ for $15 \mathrm{~min}$. Silane aldehyde conjugated to $\mathrm{SiO}_{2}$ surfaces was reacted with $1 \mu \mathrm{M}$ aminated probe with the $4 \mathrm{mM} \mathrm{NaCNBH}$ reducing agent in $10 \mathrm{mM}$ phosphate buffer $\mathrm{pH} 8.4$ for 2 hrs. The silane aldehyde on $\mathrm{SiO}_{2}$ surfaces is reduced to form an amide bond with the aminated probe. A Phosphorimager (Storm 860, Molecular Dynamics) was used for radioisotope detection from radiolabeled and sonicated surfaces.

\subsubsection{PNA and $\gamma$-PNA}

Two kinds of PNA probes have been used for surface functionalization. Conventional vertically tethered PNA with achiral polyamide backbone made up of repeating $\mathrm{N}$-(2-amnioethyl) glycine units, whereas the horizontally tethered PNA probes ( $\gamma$-PNA) use a substituent, usually corresponding to the side chain of a natural amino acid, inserted into the $\gamma$ position of the PNA backbone. The $\gamma$ PNA probe (designed and synthesized by Panagene) consisted of the same sequence as the conventional PNA and was designed with respect to favorable position of the $\gamma$-substituents in the backbone of the PNA. In our experiments, the PNA and $\gamma$-PNA have the same sequences and differ with three $\gamma$-substituted

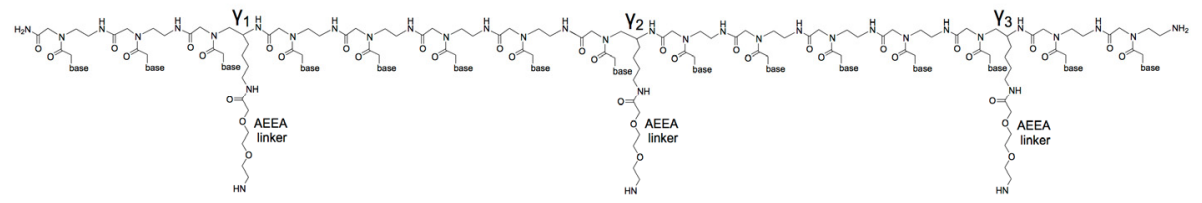

Figure 3. $\gamma$-PNA molecules with linker molecules attached at three $\gamma$-points $(\gamma 1, \gamma 2$, and $\gamma 3$ ) on the AEG-backbone with 2-aminoethoxy-2-ethoxy acetic acid (AEEA) with amine end group for attachment to $\mathrm{SiO}_{2}$ surfaces.

lysine residues in the backbone of the PNA. The PNA molecule has a single amine group available for end functionalization to surfaces in a vertical orientation. An ethylene glycol linker (AEEA) is added together with the $\gamma$ lysine residues in the PNA backbone to enhance solubility of the molecule as shown in Fig. 3.

\subsubsection{Hybridization density measurements}

$\mathrm{SiO}_{2}$ surfaces were functionalized with one of the two schemes using thiolated and aminated PNA probes and hybridized to radiolabeled complementary M0 
and non-complementary NC DNA in $1 \mathrm{mM}$ phosphate buffer with varying $\mathrm{NaCl}$ concentrations ( $1 \mathrm{mM}, 23 \mathrm{mM}$, and $137 \mathrm{mM})$. Scheme 1 shows the surface functionalization scheme of PNA with radiolabeled DNA hybridized. DNA was allowed to hybridize to the surface functionalized PNA probes for $1 \mathrm{hr}$ in static condition and in separate experiments for $10 \mathrm{~min}$ in a PDMS micro-channel with a flow rate of $7 \mu \mathrm{min}^{-1}$. For static hybridization (PDMS well based method) 10:1 PDMS was cured on a flat silicon wafer, then small area $\left(1 \mathrm{~cm}^{2}\right)$ PDMS squares were cut out and punched with a punching tool $(5 \mathrm{~mm}$ diameter, Harris Unicore). The square PDMS pieces with the hole were cleaned by sonication in ethanol, followed by assembly on $\mathrm{SiO}_{2}$ pieces as hybridization vessel. For hybridization in flow, a PDMS micro-fluidic chip with micro-channel $1 \mathrm{~cm}$ in length, $360 \mu \mathrm{m}$ in diameter was clamped on probe functionalized $\mathrm{SiO}_{2}$ surface. Radiolabeled DNA flowed using a $250 \mu$ syringe (Hamilton Gastight) with a syringe pump (PHD, Havard). The $500 \mu \mathrm{m}$ diameter inlet and outlet holes were punched into the PDMS chip with punch tool $(0.5 \mathrm{~mm}$, Harris-Unicore $)$ and a 1 $\mathrm{cm}$ long stainless steel tubing with $700 \mu \mathrm{m}$ OD could be easily make a tight fit as inlet and outlet tubing. These $700 \mu \mathrm{m}$ diameter tubing were further connected via $500 \mu \mathrm{m}$ ID Tygon tubing and connected to the syringe using $150 \mu \mathrm{m}$ ID PEEK tubing with appropriate fittings (Upchurch Scientific).

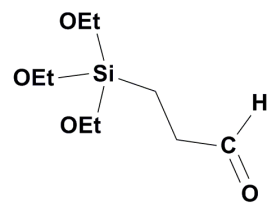

Silane aldehyde in ethanol

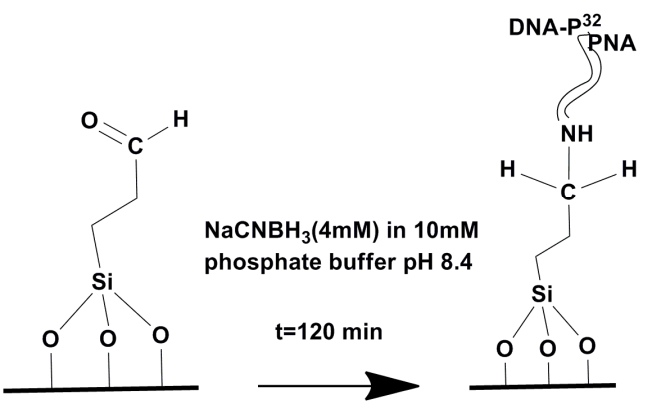

Scheme 1. DNA-P32 hybridized to aminated-PNA $\mathrm{SiO}_{2}$ surfaces.

\subsubsection{Surface plasmon resonance measurements}

The real-time PNA-DNA and $\gamma$-PNA-DNA hybridization measurements were done using a commercially available imaging surface plasmon resonance (SPR) instrument (SPRImageII, GWC Instruments). The SPR measurements was done using SPR imaging substrates (SPRchip ${ }^{\mathrm{TM}}$ GWC). The PNA and $\gamma$-PNA (Fig. 4) were functionalized on the $\mathrm{Au}$ surface prior to conducting the experiments. 
Briefly, the SPR imaging substrates were first cleaned with a fresh piranha solution and subsequently rinsed with deionized water. The cleaned sensor substrates were then incubated with PNA for 12 hrs in PDMS well of $50 \mu \mathrm{l}$ volume in a humidity chamber. The cysteine on the N-end of the PNA provides the thiol group for $\mathrm{Au}$ attachment, and for the C6-SH linker from the $\gamma$-PNA provided attachment to the Au surface. Probe functionalized SPR chips were mounted on the base of a prism with index matching oil ( $n=1.72$, Cargille Labs), with the gold side facing the flow cell. Binding was monitored with the SPR imaging instrument. All PNA-DNA hybridization was monitored in 1X PBS buffer with $\mathrm{pH} 7.4$.

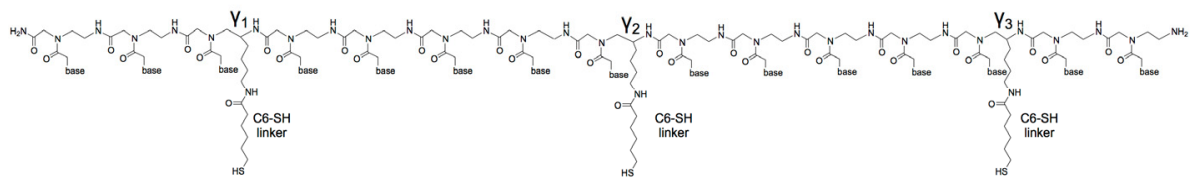

Figure 4. $\gamma$-PNA molecules with linker molecules attached at three $\gamma$-points $(\gamma 1, \gamma 2$, and $\gamma 3$ ) on the AEG-backbone with hydrocarbon chain linker with thiol end group (C6-SH) for attachment to Au surfaces.

The measured real-time SPR data is used to fit to the Langmuir kinetic model for affinity parameter extraction for the PNA-DNA binding. The measured SPR data was modeled with commercial software (Scrubber, BioLogic Software).

\subsection{Results and Discussions}

\subsubsection{Vertical and horizontal PNA-DNA hybridization on $\mathrm{SiO}_{2}$ surface}

$\gamma$-PNA probe molecules have been synthesized with three $\gamma$ attachment points along the backbone of the PNA molecule with a 2-aminoethoxy-2-ethoxy acetic acid (AEEA) linker molecule, with length $1.3 \mathrm{~nm}$, terminated with an amine group, at each $\gamma$ point, (Fig. 3) that is used to conjugate the $\gamma$-PNA to a silicon dioxide $\left(\mathrm{SiO}_{2}\right)$ surface using the reduction of silane aldehyde surface groups with sodium-cyano-borohydride $\left(\mathrm{NaCNBH}_{3}\right)$. The schematic for comparing the surface hybridization modes due to the two PNA is shown in Fig. 5. Static PNADNA and $\gamma$-PNA-DNA hybridization results are shown in Fig. 6 and hybridization results of PNA-DNA and $\gamma$-PNA-DNA in buffer flow are shown in Fig. 7. 


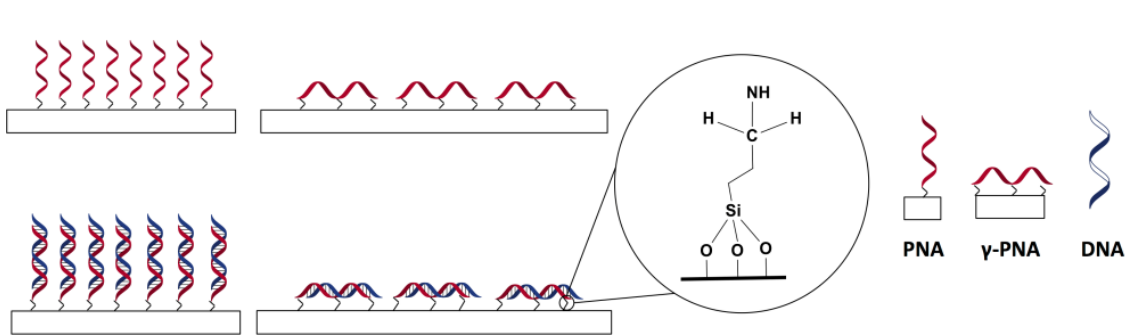

Figure 5. Schematic diagram for horizontal and vertical covalently attached aminated-PNA-DNA surface hybridization on $\mathrm{SiO}_{2}$ surfaces.

The attachment density and binding affinity of the horizontally tethered $\gamma$-PNA probes are estimated and compared to conventional vertically extended PNA probes tethered to the solid support surface with a single end attachment point. The attachment density is performed on cleaned $\mathrm{SiO}_{2}$ pieces and the PNA and $\gamma$ PNA probes are covalently attached in $1 \mu \mathrm{M}$ concentrations using a small volume reaction vessel that is clamped to the surface. The PNA and $\gamma$-PNA functionalized surfaces are exposed to complementary (M0) DNA, labeled with a $\mathrm{P}^{32}$ radioisotope for surface density estimation, in a phosphate buffer with varying ionic strength $(1 \mathrm{mM}, 23 \mathrm{mM}$, and $137 \mathrm{mM})$ at $p H 7.4$ for $1 \mathrm{hr}$ without stirring (static hybridization) in a $50 \mu \mathrm{l}$ volume reaction vessel. The posthybridization surfaces are washed with the same hybridization buffers and a final wash with high ionic strength buffer $(1 \mathrm{M} \mathrm{NaCl})$. For both cases, the posthybridization surface densities are $\sim 10^{12}$ duplexes $\mathrm{cm}^{-2}$ without significant changes for all three ionic strength buffers. A point can be made that the $\gamma$-PNA presented here with three $\gamma$ points spread across the 15-mer sequence in the backbone (Fig. 3) are not clumped together as in a chiral box, ${ }^{32,33}$ which although constrained, favor specific anti-parallel duplex ${ }^{30}$ with high ionic strength buffer. ${ }^{25}$ The post-hybridization surface densities are similar for the case of flowing the radiolabeled M0 DNA over the PNA functionalized surfaces for $10 \mathrm{~min}$. Additionally, non-specific binding to the PNA and $\gamma$-PNA functionalized surfaces is assessed using non-complementary (NC) DNA- $\mathrm{P}^{32}$ and is strongly dependent on the conformation of attachment end groups, which will be discussed later. 


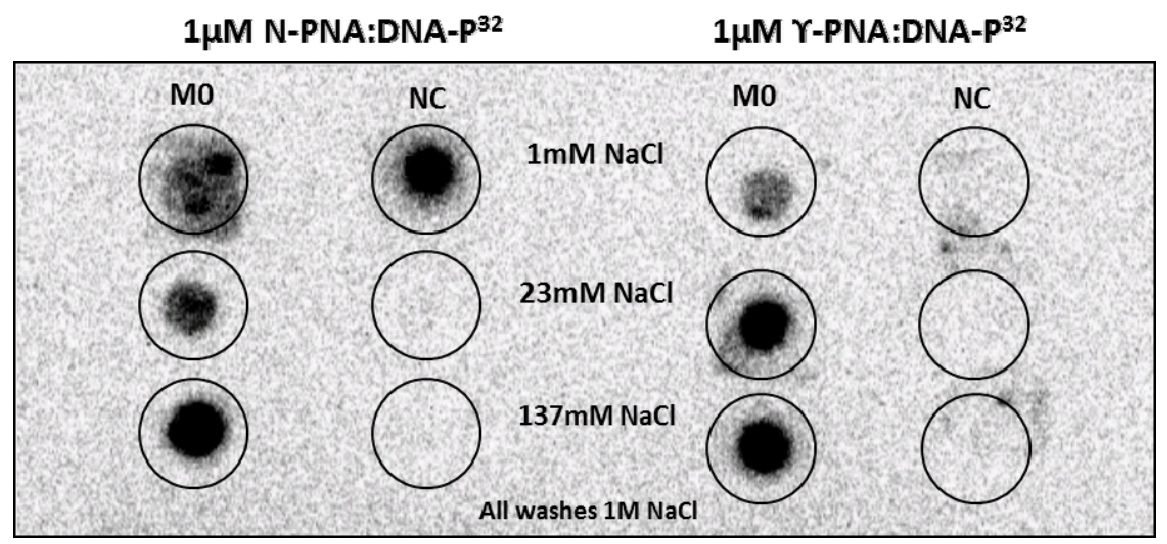

Figure 6. Static PNA-DNA-P32 and $\gamma$-PNA-DNA-P32 hybridization results for non-complementary (NC) DNA and complementary (M0).

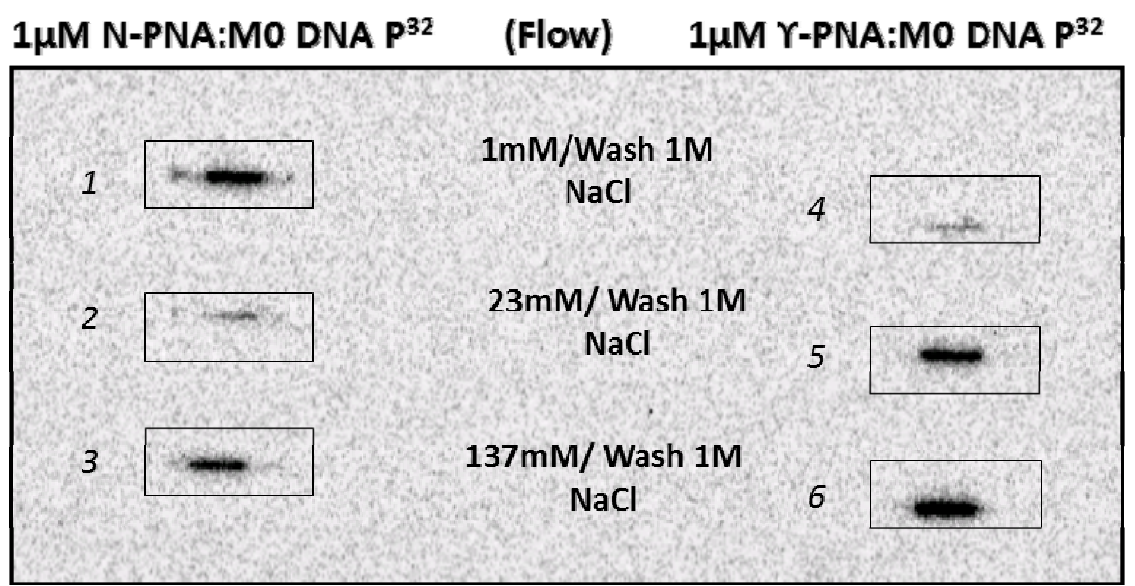

Figure 7. Flow PNA-DNA-P32 and $\gamma$-PNA-DNA-P32 hybridization results for noncomplementary (NC) DNA and complementary (M0).

\subsubsection{Vertical and horizontal PNA-DNA hybridization detection: SPR measurements}

For attachment to Au surfaces, a $\gamma$-PNA (Fig. 4) with hydrocarbon chain with thiol end group (C6-SH) linker, with length $1.1 \mathrm{~nm}$, is used to attach to gold SPR and discs and used to study the hybridization kinetics. Fig. 8 shows the surface functionalization modes of both vertical and horizontally tethered PNAs on gold surface. Kinetic monitoring of PNA-DNA with SPR is not new and have been reported before. ${ }^{25}$ An example of kinetic monitoring of $\gamma$-PNA-DNA hybridization with SPR is shown in Fig. 9. $750 \mathrm{nM}$ complementary DNA M0 
binds with a larger signal to surface attached $\gamma$-PNA. 500nM noncomplementary NC DNA clearly has very small nonspecific binding and there is no binding at all of DNA to bare gold surface. Real-time PNA-DNA and $\gamma$-PNADNA hybridization SPR measurements are shown in Fig. 10 and Fig. 11, respectively. The Langmuir single layer adsorption model is used to fit to the real-time SPR measurement data to obtain the equilibrium dissociation constant $K_{\mathrm{D}}$ for both PNA probes. The data was modeled with commercial software (Scrubber, Biologic). The extracted dissociation constants are listed in Table 2.

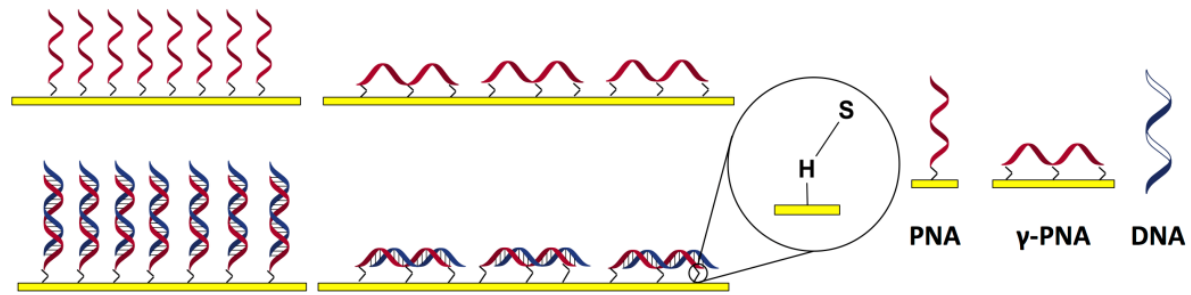

Figure 8. Schematic diagram for horizontal and vertical covalently attached thiolated-PNA-DNA surface hybridization on Gold ( $\mathrm{Au})$ surfaces.

The hybridization kinetics of the PNA-DNA and $\gamma$-PNA-DNA duplex formation are assessed by estimating the equilibrium dissociation constant $K_{\mathrm{D}}$ from real-time hybridization measurements from a conventional imaging surface plasmon resonance (SPR) instrument. Since the SPR measurement surface is $\mathrm{Au}$, the thiolated PNA and $\gamma$-PNA probes are used for attachment. The cysteine from the PNA probe provides the thiol group for attachment to the Au surface in an end-tethered vertical configuration. The $\gamma$-PNA, with C6-SH linkers attached to the three $\gamma$-points on the backbone (Fig. 4), is conjugated to the Au SPR sensors. The kinetics experiments are performed in a flow-cell with a flow velocity of $v \sim 0.5 \mathrm{~mm} \mathrm{~s}^{-1}$, thus in the reaction-limited regime, ${ }^{34}$ and the measured data is fit to the Langmuir model in two steps to estimate the association rate constant $k_{\mathrm{a}}$ $\left(\mathrm{M}^{-1} \mathrm{~s}^{-1}\right)$, which represents the speed of the second-order probe-target interaction, and the dissociation rate $k_{\mathrm{d}}\left(\mathrm{s}^{-1}\right)$, which represents the speed of the first-order breakdown of the probe-target complex. The equilibrium dissociation constant is defined as $K_{\mathrm{D}}=k_{\mathrm{d}} / k_{\mathrm{a}}(\mathrm{M})$ and a small, typically less than about $10 \mathrm{nM}, K_{\mathrm{D}}$ value indicates a high affinity of the target for the probe molecule. 


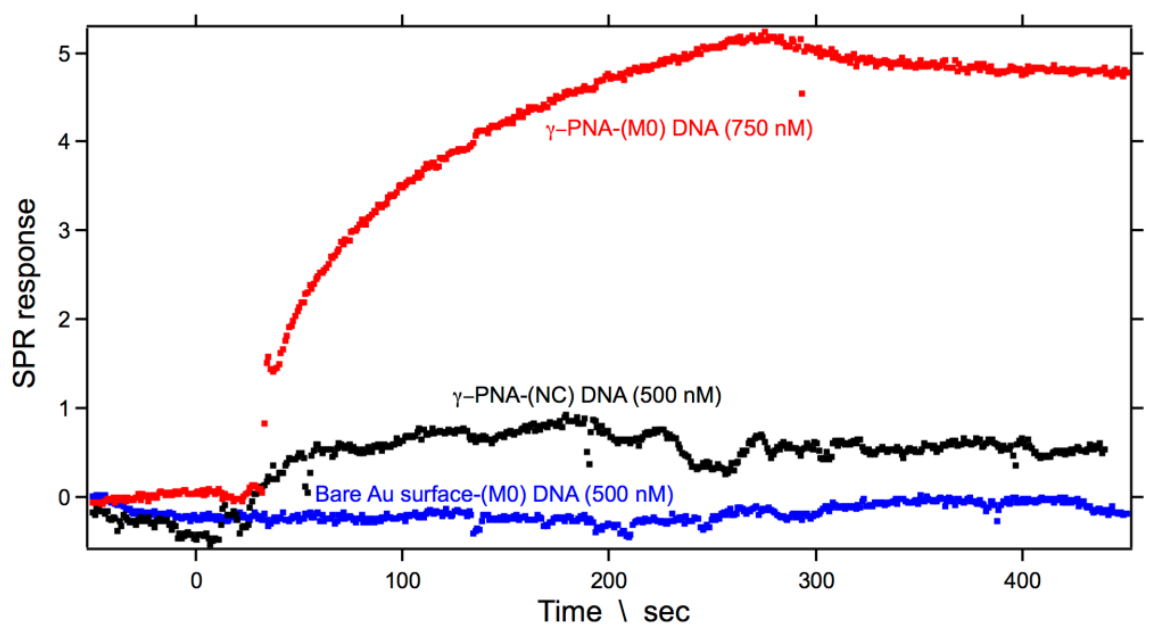

Figure 9. Examples of the DNA hybridization to complementary (M0) $\gamma$-PNA (red trace), non-complementary (NC) $\gamma$-PNA (black trace), and DNA adsorption to a bare Au surface (blue trace).

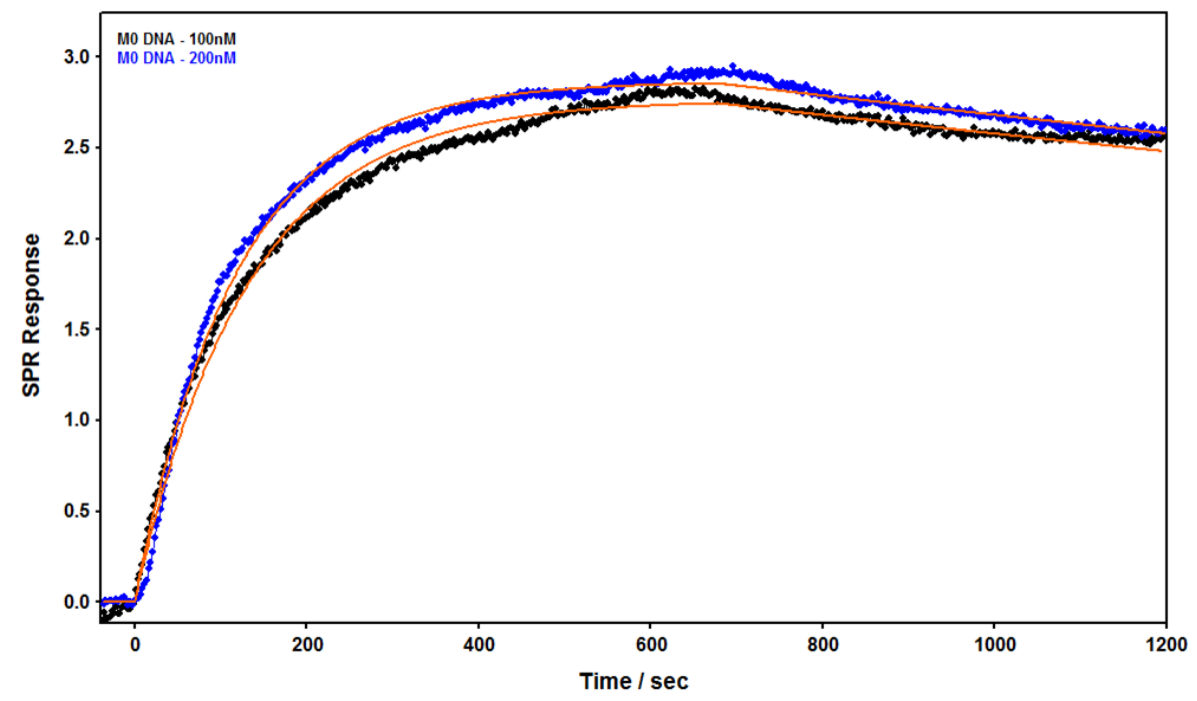

Figure 10. Real-time PNA-DNA hybridization measurements with SPR and used for $\mathrm{K}_{\mathrm{D}}$ extraction. Model traces (orange) are fit to the measured at different target DNA concentrations (blue and black traces). 


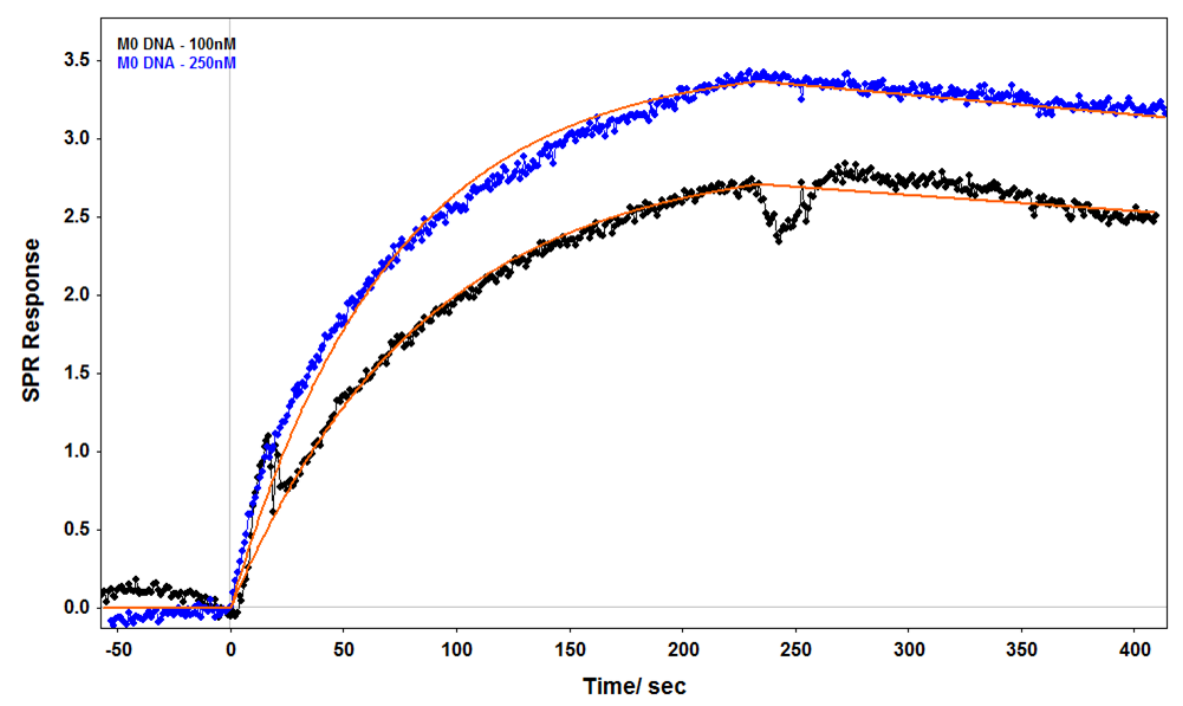

Figure 11. Real-time $\gamma$-PNA-DNA hybridization measurements with SPR and used for $\mathrm{K}_{\mathrm{D}}$ extraction. Model traces (orange) are fit to the measured at different target DNA concentrations (blue and black traces).

Table 2. Estimated PNA-DNA and $\gamma$-PNA-DNA duplex hybridization dissociation constant.

\begin{tabular}{|c|c|c|c|}
\hline Duplex & $\boldsymbol{k}_{\mathbf{a}}\left(\times \mathbf{1 0}^{\mathbf{5}}\right) \mathbf{M}^{\mathbf{- 1}} \mathbf{s}^{-\mathbf{1}}$ & $\boldsymbol{k}_{\mathbf{d}}\left(\times \mathbf{1 0}^{-4}\right) \mathbf{s}^{-\mathbf{1}}$ & $\boldsymbol{K}_{\mathbf{D}} \mathbf{n M}$ \\
\hline $\begin{array}{c}\text { PNA-DNA } \\
\text { (M0) }\end{array}$ & 0.6 & 2 & 3 \\
\hline $\begin{array}{c}\gamma \text { PNA-DNA } \\
\text { (M0) }\end{array}$ & 0.9 & 4 & 5 \\
\hline
\end{tabular}

\subsection{Conclusion}

The extracted dissociation constant for the PNA-DNA duplex is larger than the previously reported value of $1.2 \mathrm{nM}$ for 15-mer PNA-DNA duplexes attached to the Au surface with two series-connected AEEA linker molecules. ${ }^{35}$ An over estimation of the dissociation constant most likely originates from the large DNA concentrations above $100 \mathrm{nM}$ that are required to produce a signal using our SPR instrument. However, we are using the estimated $K_{\mathrm{D}}$ to compare the hybridization affinity between PNA-DNA and $\gamma$-PNA-DNA duplex hybridization. The extracted $K_{\mathrm{D}}$ for the $\gamma$-PNA-DNA duplex is larger than the conventional PNA-DNA, which is expected due to the restricted movement of 
the three-point tethered PNA probe. It has been suggested that $\gamma$-PNA-DNA duplexes would be unstable in the case of surface hybridizations with chiral PNA probes, ${ }^{36,37}$ however, in this case, the $\gamma$-PNA-DNA is slightly different from chiral PNA as it does not contain consecutive chiral points on the backbone, i.e. "chiral box". The measured $K_{\mathrm{D}} \approx 5 \mathrm{nM}$ for $\gamma$-PNA-DNA in this case is in fact significantly smaller than the reported poly-lysine-DNA-DNA horizontal surface hybridization of $K_{\mathrm{D}} \approx 200 \mathrm{nM},{ }^{26}$ and therefore, our approach is applicable for the covalent attachment of horizontally aligned PNA probes for electronic biosensors. Also due to the restricted conformation of the surface attached $\gamma$-PNA, it discriminates non-complementary DNA (NC DNA) from complementary DNA (M0) in all ionic strength situations as can be seen by comparing vertical and horizontal PNA modes in Fig. 6. 


\section{References}

1. E. M. Southern, Journal of Molecular Biology, 1975, 98, 503-517.

2. I. S. Kohane, A. Kho and A. J. Butte, Microarrays For An Integrative Genomics, M.I.T Press, 2003.

3. D. Piscevic, W. Knoll and M. J. Tarlov, Supramolecular Science, 1995, 2, 99-106.

4. J. Casey and N. Davidson, Nucleic acids research, 1977, 4, 1539-1552.

5. W. Kaiser and U. Rant, Journal of the American Chemical Society, 2010, 132, 7935- 7945.

6. G. S. Manning, Quarterly Reviews of Biophysics, 1978, 11, 179-246.

7. M. Petersen and J. Wengel, Trends in Biotechnology, 2003, 21, 74-81.

8. V. V. Demidov, Trends in Biotechnology, 2003, 21, 4-7.

9. N. Tercero, K. Wang, P. Gong and R. Levicky, Journal of the American Chemical Society, 2009, 131, 4953-4961.

10. Englund, E. A.; Appella, D. H. Org. Lett. 2005, 7, 3465-3467.

11. A. Dragulescu-Andrasi, S. Rapireddy, B. M. Frezza, C. Gayathri, R. R. Gil and D. H. Ly, Journal of the American Chemical Society, 2006, 128, 1025810267.

12. Englund, E. A.; Wang, D.; Fujigaki, H.; Sakai, H.; Micklitsch, C. M.; Ghirlando, R.; Martin-Manso, G.; Pendrak, M. L.; Roberts, D. D.; Durell, S. R.; Appella, D.H. Nat. Commun. 2012, 10, 614-620.

13. M. Egholm, O. Buchardt, L. Christensen, C. Behrens, S. M. Freier, D. A. Driver, R. H. Berg, S. K. Kim, B. Norden and P. E. Nielsen, Nature, 1993, 365, 566-568.

14. P. E. Nielsen, M. Egholm, R. H. Berg and O. Buchardt, Science, 1991, 254, 1497-1500.

15. S. Tomac, M. Sarkar, T. Ratilainen, P. Wittung, P. E. Nielsen, B. Norden and A. Graslund, Journal of the American Chemical Society, 1996, 118, 55445552 .

16. J. C. Hanvey, N. J. Peffer, J. E. Bisi, S. A. Thomson, R. Cadilla, J. A. Josey, D. J. Ricca, C. F. Hassman, M. A. Bonham, K. G. Au, S. G. Carter, D.A. Bruckenstein, A. L. Boyd, S. A. Noble and L. E. Babiss, Science, 1992, 258, 1481-1485.

17. K. E. Lundin, L. Good, R. Strömberg, A. Gräslund and C. I. E. Smith, 2006, vol. 56, pp. 1-51. 
18. B. S. Gaylord, M. R. Massie, S. C. Feinstein and G. C. Bazan, Proceedings of the National Academy of Sciences of the United States of America, 2005, 102, 34-39.

19. J. I. Hahm and C. M. Lieber, Nano Letters, 2004, 4, 51-54.

20. G. J. Zhang, G. Zhang, J. H. Chua, R. E. Chee, E. H. Wong, A. Agarwal, K. D. Buddharaju, N. Singh, Z. Gao and N. Balasubramanian, Nano Letters, 2008, 8, 1066-1070.

21. De, J. van Nieuwkasteele, E. Carlen and A. van den Berg, Analyst, 2013. 138, 2013, 3221-3229.

22. M. H. Sørensen, N. A. Mortensen and M. Brandbyge, Applied Physics Letters, 2007, 91.

23. E. Stern, R. Wagner, F. J. Sigworth, R. Breaker, T. M. Fahmy and M. A. Reed, Nano Letters, 2007, 7, 3405-3409.

24. R. Elnathan, M. Kwiat, A. Pevzner, Y. Engel, L. Burstein, A. Khatchtourints, A. Lichtenstein, R. Kantaev and F. Patolsky, Nano Letters, 2012, 12, 52455254.

25. H. Park, A. Germini, S. Sforza, R. Corradini, R. Marchelli and W. Knoll, Biointerphases, 2007, 2, 80-88.

26. Y. L. Bunimovich, Y. S. Shin, W. S. Yeo, M. Amori, G. Kwong and J. R. Heath, Journal of the American Chemical Society, 2006, 128, 16323-16331.

27. J. Fritz, E. B. Cooper, S. Gaudet, P. K. Sorger and S. R. Manalis, Proceedings of the National Academy of Sciences of the United States of America, 2002, 99, 14142-14146.

28. S. Chen, J. G. Bomer, E. T. Carlen and A. van den Berg, Nano Letters, 2011, 11, 2334-2341.

29. S. Pensato, M. Saviano and A. Romanelli, Expert Opinion on Biological Therapy, 2007, 7, 1219-1232.

30. A. Dragulescu-Andrasi, P. Zhou, G. He and D. H. Ly, Chemical Communications, 2005, 244- 246.

31. F. Patolsky, G. Zheng and C. M. Lieber, Nature Protocols, 2006, 1, 17111724.

32. R. Corradini, G. Feriotto, S. Sforza, R. Marchelli and R. Gambari, Journal of Molecular Recognition, 2004, 17, 76-84.

33. Manicardi, A. Calabretta, M. Bencivenni, T. Tedeschi, S. Sforza, R. Corradini and R. Marchelli, Chirality, 2010, 22, E161-E172. 
34. M. Zimmermann, E. Delamarche, M. Wolf and P. Hunziker, Biomedical Microdevices, 2005, 7, 99-110.

35. A. Cattani-Scholz, D. Pedone, F. Blobner, G. Abstreiter, J. Schwartz, M. Tornow and L. Andruzzi, Biomacromolecules, 2009, 10, 489- 496.M. Masuko, Nucleic acids research. Supplement (2001), 2003, 145-146.

36. J. Weiler, H. Gausepohl, N. Hauser, O. N. Jensen and J. D. Hoheisel, Nucleic Acids Research, 1997, 25, 2792-2799. 


\section{Chapter 8}

\section{Conclusions and Outlook}

\subsection{Conclusions}

This thesis describes the design, implementation and characterization of a bioassay for realization in the Nanopill, an ingestible automated diagnostic pill for the detection of free floating hyper-methylated DNA (hm-DNA) with gene sequence consistent for colorectal cancer, the cancer marker considered in this work. The thesis describes the development and characterization of an hm-DNA enrichment microchip, and the electronic detection of DNA using silicon nanowire (Si-NW) biosensors, as part of a two-step assay for cancer marker detection that can be eventually integrated into the Nanopill system.

Free-floating hm-DNA is present in very low concentrations in biological fluids such as sputum, urine, blood and gastrointestinal fluid. Since a non-amplification based detection system is used for detection, the hm-DNA cancer marker is captured and pre-concentrated prior to detection. The preconcentration system has been designed and implemented using a pillar-based glass-silicon microfluidic chip for the solid-phase extraction of hm-DNA. The pillar extraction surfaces are coated with a commercially available methyl binding domain (MBD) protein that specifically captures double-stranded hmDNA in the $\mathrm{CpG}$ island hyper-methylation context. The solid-phase MBD microchip is designed for the capture and elution of hm-DNA with quantities less than $1 \mathrm{ng} \mathrm{ml}^{-1}$. Conventional silane-based attachment chemistry was used to conjugate the MBD protein to the pillar arrays. A surface plasmon resonance biosensor was used to characterize the hm-DNA capture and compared to nonhyper-methylated DNA. A $28 \times \mathrm{hm}$-DNA enrichment factor is demonstrated using a $25 \mu \mathrm{L}$ elution volume and a capture-elution efficiency greater than $90 \%$, which is consistent with commercially available large volume magnetic bead based assays. 
The DNA detection assay is based on Si-NW biosensors that are integrated into a microfluidic auto-sampler system designed and implemented for automated sample delivery to the sensors. The microfluidic auto-sampler system eliminates erroneous sensor signals during sample switching caused by surface potential variations that are induced by a streaming potential from the flowing low ionic strength sample buffer. The Si-NWs detect the surface potential change due to surface charge density changes on the surface of the gate-oxide. This principle has been used to detect the negative electronic charge on DNA backbone using specific base pairing to complementary probes attached to Si-NW surfaces. Since low ionic strength buffers are important to reduce counter-ion screening, peptide nucleic acid (PNA) probe molecules are used for the DNA hybridization detection. The gate-oxide of the Si-NW biosensors was terminated with the PNA probes using a silane-based attachment chemistry. The surface attachment chemistry protocols, surface attachment density, and nonspecific adsorption problems of the PNA molecules were carefully studied using fluorescence and radioactivity labeled assays. Real-time DNA-PNA measurements were fit to a conventional Langmuir model, which resulted in an equilibrium hybridization association constant $K_{\mathrm{A}} \approx 7 \times 10^{6} \mathrm{M}^{-1}$, which is small compared to previously reported values. The low extracted affinity could be attributed to target depletion effects due to the high non-specific absorption of PNA probes as confirmed by our radioactive studies.

The Si-NW sensors were further improved by implementing a differential measurement configuration, which resulted in a $30 \times$ reduction in the drift of the measured Si-NW response. Furthermore, the differential configuration was extended to include arrays of parallel Si-NW that increase the sensing surface area while maintaining the high sensitivity of the Si-NW sensors. Finally, a covalent horizontally tethered $\gamma$-PNA probe has been conceived, implemented, and $\gamma$-PNA-DNA hybridization was demonstrated. The horizontal $\gamma$-PNA-DNA and conventional vertical PNA-DNA equilibrium dissociation constants are comparable and offer an important improvement for Si-NW based DNA hybridization detection in high ionic strength buffers. The research goals for the Nanopill assay have been demonstrated in principle and various solutions to identified problems offered. The microfluidic solid phase hm-DNA enrichment system and the electronic detection of DNA are functional and promising for further application in a single platform two-step assay. 


\subsection{Outlook}

\subsubsection{Si-NW measurement of horizontal $\boldsymbol{\gamma}$-PNA-DNA}

In Chapter 7, the feasibility of horizontal versus vertical PNA-DNA hybridization detection has been demonstrated. A low ionic strength buffer produces less counter-ion screening of target DNA, which is advantageous for Si-NW biosensors, compared to a buffer with ionic strength consistent physiological conditions $(\sim 140 \mathrm{mM})$, where DNA-DNA duplex formation is optimal. The horizontal $\gamma$-PNA-DNA duplex will be less affected by counter-ion screening than the conventional vertical configuration. Both vertical PNA-DNA duplexes and horizontal $\gamma$-PNA-DNA duplexes hybridize more specifically with higher ionic strength buffers. In addition, $\gamma$-PNA could prevent non-specific binding of non-complementary DNA in a low ionic strength buffer.

Chapter 5 describes the real-time detection of vertical PNA-DNA hybridization with Si-NWs with a limit of detection of $50 \mathrm{nM}$, which is dependent on the assay test conditions, e.g. flow velocity, probe surface density, assay time, etc. A differential measurement mode of Si-NW was implemented which could improve the limit of detection further. The quiescent output signal in this case is nulled to zero and so that increased sensitivity in the sensor signal would be detected due to a reduced detection range of the conductance measurement instrument.

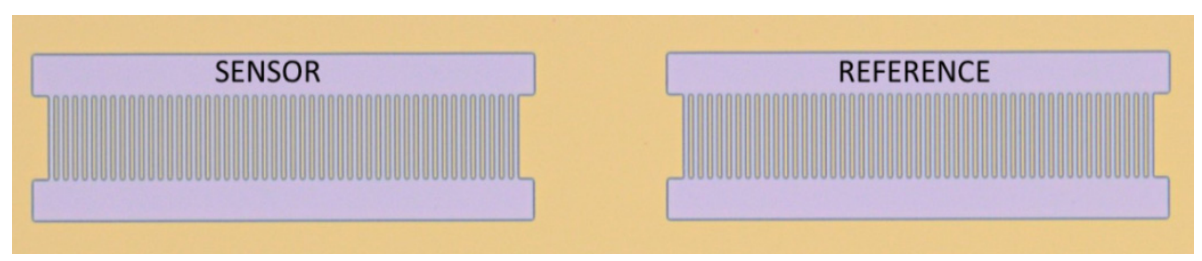

Figure 1. Sensor and reference Si-NW arrays for differential measurement.

A subsequent device design with two banks of parallel Si-NW arrays connected in differential mode, which consists of the sensor array and reference array, where each bank contains $N$-parallel connected Si-NW sensor (Fig. 1) such that the differential response is amplified, by a factor of $N \times$ using the measurement configuration shown in Fig. 2. The horizontal $\gamma$-PNA-DNA hybridization in high ionic strength buffer would be a good application to be tested with the differential Si-NW sensor arrays for reducing the DNA detection limit and eventually real-physiological relevant samples. 


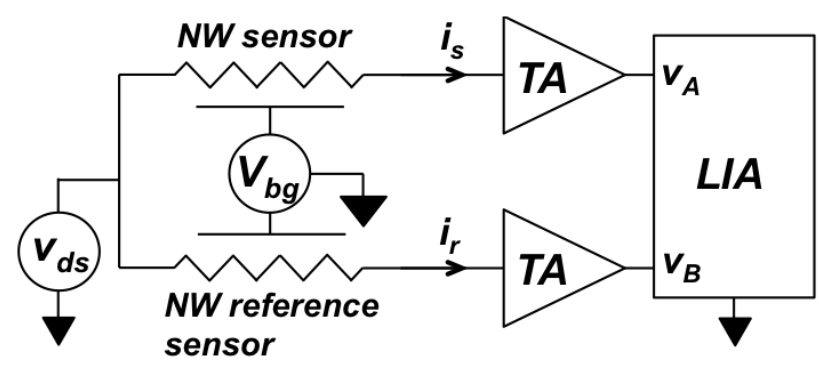

Figure 2. Si-NW sensor and reference arrays for differential measurement.

\subsubsection{Combined hyper-methylation DNA enrichment and electronic DNA detection}

In Chapter 4, a solid phase hyper-methylated DNA (hm-DNA) capture-elution microchip, which is based on a specific methyl-binding domain (MBD) protein conjugated to a micromachined silicon post array, has been demonstrated. The capture-elution protocol involves the capture step using a $160 \mathrm{mM} \mathrm{NaCl}$ buffer and elution step with a $2 \mathrm{M} \mathrm{NaCl}$ buffer. According to the Methyl-Miner protocol (Invitrogen), the elution can be varied as a gradient elution depending on the level of hyper-methylation present in the sample, by varying the buffer strength from $200 \mathrm{mM}$ up to $2 \mathrm{M}$ using a single one-shot elution of the entire hm-DNA. The gradient elution with hm-DNA on the MBD functionalized silicon pillar chips need to be studied carefully, such that a single platform hmDNA capture-elution and subsequent direct Si-NW detection can be possible with the high salt compatible horizontal $\gamma$-PNA probes based detection on SiNW sensors.

The Nanopill operation can be realized with the sample valve (SV) open for hm-DNA capture at the MBD microchip with continuous intestinal fluid pumping, followed by SV closure and elution valve (EV) open and output from elution chip directed onto Si-NW functionalized $\gamma$-PNA for allowing horizontal high ionic strength specific DNA hybridization detection, as shown in Fig. 3. 


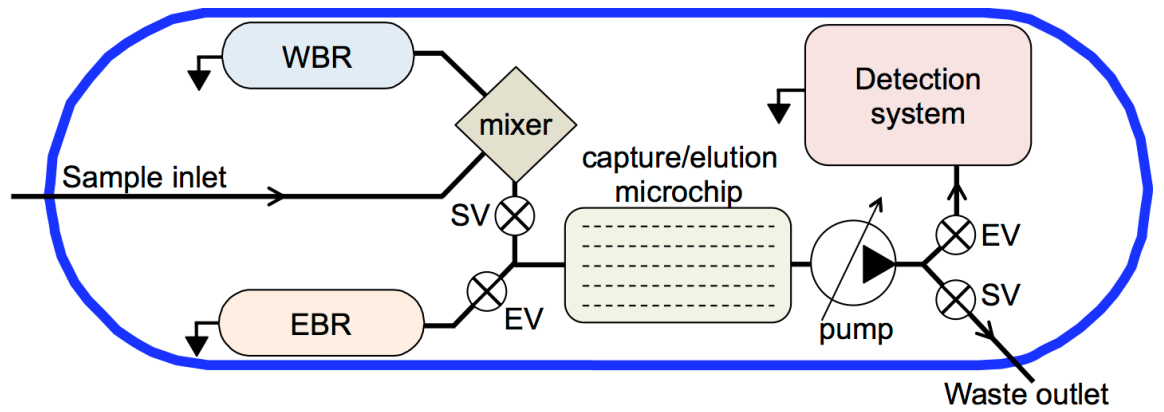

Figure 3. Nanopill complete with capture-elution and Si-NW based hm-DNA detection scheme. 


\section{Samenvatting}

Het Nanopil-project is gefinancierd door een particuliere Nederlandse kankeronderzoeksstichting en ook voor een deel uit de Spinozapremie, die de NWO in 2009 heeft toegekend aan professor Albert van den Berg van de Universiteit Twente. Het Nanopil-project betreft een ambitieus en uitdagend onderzoek met als doel de ontwikkeling van een diagnostische pil die in staat is om de ontwikkeling van darmkanker bij mensen in een zeer vroeg stadium te detecteren. Een ingenomen Nanopil doorloopt het maag-darmkanaal en neemt onderweg vloeistofsamples van de darminhoud en test deze monsters op aanwezigheid van speciale tumormarkers; in het geval van de Nanopil wordt getest op het vrij voorkomen van hypergemethyleerd DNA (hm-DNA) met een gensequentie die specifiek is voor dikkedarmkanker. Uiteindelijk zal de gebruiker direct worden gewaarschuwd door de Nanopil, als er aanwijzingen zijn voor de aanwezigheid van de bewuste tumormarker. Het moge duidelijk zijn dat de ontwikkeling van een volledige Nanopil niet binnen een enkel promotieonderzoek valt te realiseren. Dit proefschrift beperkt zich tot de ontwikkeling en fabricage van een geminiaturiseerde biosensor op chip, voor het automatisch invangen en verrijken van de hm-DNA tumormarker en de daaropvolgende detectie van de voor dikkedarmkanker specifieke DNAsequentie, gebruikmakend van silicium nanowires ( $\mathrm{Si}-\mathrm{NW}$ ) die rechtstreeks uitgelezen kunnen worden, zonder amplificatiestap.

Ongebonden hm-DNA is in zeer lage concentraties aanwezig in verschillende biologische samples, zoals ontlasting, sputum, urine, bloed en darmvloeistof. Daar er is gekozen voor een detectiesysteem zonder amplificatie, moet de ingevangen hm-DNA tumormerker eerst geconcentreerd worden, voordat deze gedetecteerd kan worden. Het deel van de sensor dat hiervoor is ontworpen, bestaat uit een microfluidische chip gemaakt van silicium en glas, met daarin een grote array van pilaartjes die zorgen voor de vastefase-extractie van hm-DNA. Het oppervlak van de pilaartjes in de array die het hm-DNA binden, wordt gecoat met een commercieel verkrijgbaar methyl bindend domein (MBD) eiwit, dat specifiek dubbelstrengs hm-DNA invangt in het hypergemethyleerde CpG-gebied. Het MBD-extractie deel van de chip is ontworpen voor het invangen en uitwassen van hm-DNA bij concentraties onder de $1 \mathrm{ng} / \mathrm{ml}$. De MBD-eiwitten worden met conventionele op silaan gebaseerde oppervlaktechemie aan de pilaartjes gebonden. Het invangen van hm-DNA is 
gekarakteriseerd met behulp van een speciale surface plasmon resonantie (SPR) biosensor. Hiermee is een 28 -voudige toename in concentratie van hm-DNA aangetoond, in een uiteindelijk volume van $25 \mu \mathrm{l}$. Dit komt overeen met een invang- en uitwasefficiëntie van meer dan $90 \%$, hetgeen vergelijkbaar is met commercieel verkrijgbare systemen die gebruik maken van magnetische deeltjes, maar op veel grotere sample volumes werken.

Het DNA-detectie deel van de chip is gebaseerd op Si-NW sensoren, die zijn geïntegreerd in het microfluïdische auto-sampler systeem van de chip, dat zorgt voor de automatische toevoer van vloeistofsamples naar de sensoren. Het auto-sampler systeem onderdrukt foutsignalen tijdens het wisselen van de samples, die worden veroorzaakt door veranderingen van de oppervlaktepotentiaal als gevolg van het instromen van de buffervloeistof. De Si-NW's detecteren fluctuaties in oppervlakte-potentiaal doordat de ladingsdichtheid op het oppervlak van het gate-oxide verandert. Dit principe wordt ook gebruikt voor de detectie van de negatieve lading op de DNAbackbone, met behulp van specifieke baseparing aan complementaire probes die zich op het gate-oxide oppervlak bevinden. Daar bufferoplossingen met een lage ionconcentratie belangrijk zijn voor het tegengaan van 'counter-ion screening', worden peptide nucleïnezuur (PNA) moleculen gebruikt voor de detectie van DNA-hybridisatie. Het gate-oxide van de Si-NW biosensoren is bedekt met PNA probe-moleculen, eveneens gebruik makend van silaan oppervlaktechemie. Het proces achter de koppeling van PNA aan het gate-oxide en de resulterende molecuuldichtheid zijn uitgebreid bestudeerd, alsmede het probleem van de nietspecifieke adsorptie van het PNA, onder meer door gebruik te maken van fluorescentie en radioactief gelabelde preparaten. Real-time DNA-PNA metingen zijn uitgezet tegen een conventioneel Langmuir model en dit resulteerde in een evenwichtsconstante voor de hybridisatie $\left(K_{\mathrm{A}} \approx 7 \times 10^{6} \mathrm{M}^{-1}\right)$ die vrij laag is vergeleken met eerdere gepubliceerde waarden. Deze lage waarde voor de affiniteit zou veroorzaakt kunnen worden door de verarming van het targetoppervlak als gevolg van de hoge niet-specifieke absorptie van PNA probe-moleculen, die wordt bevestigd door ons radioactieve onderzoek.

De implementatie van een differentiemeting in de opstelling heeft een verdere verbetering gegeven van de Si-NW sensoren, door een 30-voudige reductie van de drift in de Si-NW-metingen. Tevens werd deze verschilmeting uitgebreid met het aansluiten van parallelle Si-NW's, waardoor het 
sensoroppervlak wordt vergroot, terwijl de hoge gevoeligheid van de Si-NW's blijft gehandhaafd. Tot slot zijn er experimenten gedaan met horizontaal gebonden $\gamma$-PNA probe-moleculen en $\gamma$-PNA-DNA-hybridisatie is hierbij aangetoond. De horizontale $\gamma$-PNA-DNA en de verticale $\gamma$-PNA-DNA dissociatieconstanten hebben vergelijkbare waarden en bieden een belangrijke mogelijkheid tot verbetering van de op Si-NW's gebaseerde detectie van DNAhybridisatie in bufferoplossingen met hogere ion sterktes.

De onderzoeksdoelen voor de Nanopil zijn in principe gehaald, de werking is gedemonstreerd en voor alle geconstateerde problemen zijn oplossingen aangedragen. Het microfluïdische hm-DNA verrijkingsdeel en het elektronische deel voor detectie van het DNA zijn functioneel en lijken veelbelovend bij toepassing in een alleenstaande tweetraps detectie chip. 


\section{Acknowledgment}

This is the best part of the thesis writing. This extremely challenging project regarding bio-assay for 'Nanopill' application could only be completed by the magnanimous support and guidance of wonderful people I worked with. I want to express my sincere appreciation and gratitude to Prof Albert van den Berg and Dr Edwin Carlen for choosing me for this research position and allowing me ample space for learning different aspect of microtechnology and biotechnology. Edwin has been my daily supervisor and I am so happy that you were very patient with me as it took so long for me to realize the DNA detection on SiNWs. Every meeting, every discussion, planning of experiments, experimental set-up, poster-making, I learnt a lot from you. This has groomed me, as I had Biotechnology training and had no experience of such an interdisciplinary research. It was an intense working experience and I have learned a lot. I have improved in every aspect of research, I believe. I owe a great deal to you Edwin for the empathetic mentoring I received during my difficult times in the last five years, helping me as I learnt to grow up, and living by myself away from home in India. My heartfelt thanks to you. I am glad I got the opportunity to be a visiting PhD student at Karolinska University Hospital, Stockholm in the proteomics lab of Dr Serhiy Souchelnytskyi to perform surface density studies of probe attachment with radiolabelling. It was a very nice experience for both work and stay. I am happy the work I did there was successful and added to our Nanopill detection research. I want to thank you Serhiy for giving me ample freedom to work in the laboratory, although I was a visiting student for 3 months. Sanaz, Ola, Alena, Magda, Simone, and Celine, thank you so much my lovely lab-mates for an amazing time in Karolinska.

Jan van Nieuwkasteele, has been providing me the fabricated Si-NW chips. I am immensely thankful to you Jan, that I did not have to undertake this complex task myself. I fabricated the passive silicon-glass MBD capture chips and realized how much trouble it could be. Thank you Jan for the ready-to-use Si-NW chips. The MBD silicon-glass chips I could fabricate, with constant help and inputs from Wouter (Sparreboom), Lennart and Johan. Thank you so much Lennart, Wouter and Johan. Songyue and Justyna I owe you a lot for training me on the Si-NW measurement set-up, wire-bonding, and using the Nanoenabler respectively. Thanks a lot Songyue. Thanks a lot Justyna. Paul many thanks to you for all the ordering of chemicals and supplies. You made it working for me 
both here at BIOS and also during my time in Sweden. Hermine, you have always taken care of everything from arrival visa, housing, leave, flights, and hotel bookings. It is really helpful to receive this assistance and settle down when we newly arrive from a different country. Especially I remember how you took care of my health insurance when I was hospitalized due to an accident in my first 10 days of arrival. Thank you so much Hermine. I want to thank everyone from BIOS for helping me out in that time. Evelien, Severine, Eddy, Ganesh, Tamalika, Pramod, Peteji, Ling Ling, Ming Liang, and Masood. You were all very kind to me, helping with every detail, translating medical documents from Dutch to English, providing me food, release from hospital and taking care of travelling back to India for my recovery. I also cannot thank enough for approval of the 3-month care leave in my $2^{\text {nd }}$ year of $\mathrm{PhD}$, enabling me to support my family in a time of serious crisis. I realize the value of doing my $\mathrm{PhD}$ as an employee at the University with sound employee welfare and interests accounted for in the employment scheme.

It has been amazing to do my $\mathrm{PhD}$ in the BIOS group. The fantastic opportunity to visit research groups abroad as part of our Work-Weeks in United States and France is something very unique. I am so grateful for generous funding for all conference visits in Montreux, Okinawa and Barcelona as well as for all other work related costs. The Friday afternoon talks, the coffee-breaks for cake, and barbeques at Albert's place complements very well to the wonderful research experience at BIOS. My office-mates Iris, Ganesh and Rerngchai in Hogekamp and later in Carre, Maarten, Fleur, Aditya, JP, Henriette, Allison, Loan, and Wei-Shu, I thank you for your company, discussions and laughter together. My other colleagues at BIOS Yanbo, Zhenxia, Yawar, Sourav, Mathieu, Loes, Burcu, Wesley, Floris, Laura, Susan, Rogier, Bjorn, Liza, Alexander, Sertan, and Trieu I also want to thank you for your company and making BIOS, such a wonderful place to work. My apologies, if I have missed out in mentioning someone. My friends Nobina, Sumy, Bano, Nupur di, and Tamalika di thank you so much for all the warmth and friendship during these PhD years in Netherlands.

It was not easy to be alone in Netherlands as a $\mathrm{PhD}$ student. Bedabrata, your arrival for pursuing your post-doc position at Philips in Eindhoven has been the greatest blessing for me. I have started enjoying my living in Netherlands ever since a lot better. You took care of me every-weekend 
travelling to Enschede, restored a sense of love and care I so missed, living away from family. Our relationship has grown stronger and now we are planning our marriage after my graduation. I cannot thank you enough. Love you.

Mom and Dad I express all my love and gratitude for bringing me up with such strong values of perseverance, hard work and belief in me that I would complete the task I have undertaken. I so miss you Ma that you cannot be here to witness this in person. I wish Baba could make the visit to Netherlands as well. My sisters Aditi and Archita, fondly 'Didi' \& 'Dolly', my brother-in-law Subhro da and my cute little niece Pihu, I miss you all on this occasion of my graduation. Nevertheless the good news is here, that my $\mathrm{PhD}$ research is completed, I am graduating and will be back to be with all of you at home in India very soon! My old buddies Priyas, Sanju, Sid, Chandreyee, Shanky, Panchali, Shaoli, Debamitra, Sayani, Dipu, Chutki, Joyee, and all my M.Tech buddies...love you very much. Thank you so much for our inspiring friendship.

Last but not the least; I want to acknowledge the faith in divine that if nature brings it to you, it would also provide for you to cross over it. One just needs to hold on to faith, come what may. All my meditation and yoga buddies from Art of Living, Bedabrata, Subi da, Banani didi, Balkrishna, Suraj, Shikha, we are a family now. Pia, Lisbeth, Niraj, Forouzan and Pamela you were my blessing as I got connected with Art of Living, Stockholm during my work-trip. It was amazing to stay with you and spend time together. The innumerable trips to Bad Antogast in Oppenau, and Aachen, meditating with all of you has always helped me to reconnect with my inner strength and kept me going. I thank you so much.

Thank you everyone, for making this part of my life so wonderful and abundant with all your presence. I had a great adventure earning my $\mathrm{PhD}$ !

I love you all. 


\section{Appendix}

\section{List of Symbols}

$R_{\text {hyd }} \quad$ Hydraulic resistance [Pa $\left.\mathrm{S} \mathrm{m}^{-3}\right]$

$v_{\mathrm{s}} \quad$ Flow velocity $\left[\mathrm{m} \mathrm{s}^{-1}\right]$

$Q \quad$ Volumetric flow rate $\left[\mathrm{m}^{3} \mathrm{~s}^{-1}\right]$

$\mu \quad$ Dynamic viscosity of the sample [Pa s]

Re Reynolds number

$C_{o x} \quad$ Front gate oxide capacitance per unit area $\left[\mathrm{F} \mathrm{m}^{-2}\right]$

$E_{F} \quad$ Fermi level

$E_{\text {ref }} \quad$ Reference electrode potential [ $\left.\mathrm{mV}\right]$

$\varepsilon_{o x} \quad$ Relative permittivity of oxide $\left[\mathrm{F} \mathrm{m}^{{ }^{l}}\right]$

$\varepsilon_{S i} \quad$ Relative permittivity of silicon $\left[\mathrm{F} \mathrm{m}^{-1}\right]$

$f_{d} \quad$ Depletion width [m]

$G_{c} \quad$ Conductance of $\mathrm{Si}-\mathrm{NW}[\mu \mathrm{S}]$

$g_{m} \quad$ Transconductance [S]

$N_{a} \quad$ Impurity doping concentration $\left[\mathrm{cm}^{-3}\right]$

$\psi_{\mathrm{o}} \quad$ Potential at the gate-oxide surface [V]

$q \quad$ Electronic charge $[C]$

$Q_{f} \quad$ Fixed charge near $\mathrm{Si} / \mathrm{SiO}_{2}$ surface [C]

$Q_{s s} \quad$ Interface states near the gate-oxide/silicon interfaces

$\chi^{\text {sol }} \quad$ Surface dipole potential of the sample solution [V]

$\mu_{\mathrm{a}} \quad$ Field dependent hole mobility in the accumulated layer [ $\left.\mathrm{cm}^{-2} V \mathrm{~s}\right]$

$\mu_{\mathrm{b}} \quad$ Dopant concentration dependent bulk hole mobility $\left[\mathrm{cm}^{-2} \mathrm{Vs}\right]$

$V_{\mathrm{fb}} \quad$ Flat-band voltage [V]

$V_{\text {fg }} \quad$ Front-gate voltage $[V]$

$v_{d s} \quad$ Drain-source voltage [V]

$V_{b g} \quad$ Back-gate voltage [V]

$c_{o} \quad$ Ionic strength [M]

$i_{f g} \quad$ Front-gate current [pA]

$i_{d s} \quad$ Drain-source current [nA]

$a_{\mathrm{H}+} \quad$ Ion activity of the solution

${ }^{\circ} a_{\mathrm{H}^{+}} \quad$ Ion activity at the point of zero charge

$\lambda_{d} \quad$ Debye length [m]

$k_{a} \quad$ Association rate constant $\left[\mathrm{Ms}^{-}{ }^{1}\right]$ 
$k_{d} \quad$ Dissociation rate constant $\left[s^{-1}\right]$

$K_{D} \quad$ Equilibrium dissociation constant [M]

$K_{A} \quad$ Equilibrium association constant $\left[\mathrm{M}^{-1}\right]$

$R_{\max } \quad$ Maximum sensor signal response

$N_{p} \quad$ Probe density [molecules $\mathrm{cm}^{-2}$ ]

$[C]_{i} \quad$ Concentration of the target analyte [M]

$\sigma_{o x} \quad$ Silicon oxide charge density $\left[\mathrm{C} \mathrm{m}^{-2}\right]$

$\sigma_{D N A}$ Charge density of DNA $\left[\mathrm{C} \mathrm{m-}^{2}\right]$

Da Damkohler number

$\tau_{\mathrm{r}} \quad$ Time constant [s]

$\Delta R \quad$ Change in silicon nanowire resistance [ $\Omega]$ 


\section{List of Publications}

\section{Peer reviewed journal publications}

1. A. De, S. Souchelnytskyi, A. van den Berg and E. T. Carlen, "PNA:DNA hybridization: A comparison of vertical and horizontal surface hybridization due to PNA backbone modification”, ACS Appl. Mater. Interfaces, 2013, 5, 4607-4612.

2. A. De, J. van Nieuwkasteele, E. T. Carlen and A. van den Berg, "Integrated label-free silicon nanowire sensor arrays for (bio)chemical analysis" Analyst, 2013, 138, 3221 - 3229.

3. A. De, W. Sparreboom, L. de Vreede, E. T. Carlen and A. van den Berg, "Small volume hypermethylated DNA enrichment for epigenetics" manuscript in preparation.

\section{Book chapter}

1. A. De, S. Chen and E.T. Carlen "Probe-free nanowire platforms for Biosensing" in Semiconducting silicon nanowires for biomedical applications, Woodhead Publishing, May 2013.

\section{International conferences}

1. A. De, J. van Nieuwkasteele, E. T. Carlen and A. van den Berg, "Real-time measurements of DNA:PNA hybridization kinetics with silicon nanowire biosensors", The 17th International Conference on Solid-State Sensors, Actuators and Microsystems (Transducers 2013 and Eurosensors XXVII) (Oral presentation).

2. A. De, W. Sparreboom, L. de Vreede, E. T. Carlen and A. van den Berg, "Small volume hypermethylated DNA enrichment for epigenetics", The $16^{\text {th }}$ International Conference on Miniaturized Systems for Chemistry and Life Sciences 2012.

3. A. De, S. Chen, J. van Nieuwkasteele, E. T. Carlen and A. van den Berg "Silicon nanowire biosensors: Applications to $\mathrm{pH}$ sensing and DNA hybridization detection”, Nanobiotech Montreux 2011.

4. A. De, S. Chen, J. van Nieuwkasteele, W. Sparreboom, E. T. Carlen and A. van den Berg, "Integrated lab-on-a-chip silicon nanowire biosensing platform", Micro Mechanics Europe Workshop 2010. 
